\title{
Rehabilitatives Krafttraining zur Behandlung tumorassoziierter Fatigue unter Einbeziehung zirkadianrhythmischer Aspekte während und nach stationärer onkologischer Rehabilitation
}

\author{
DISSERTATION \\ zur Erlangung des Doktorgrades \\ der Sozialwissenschaftlichen Fakultät \\ der Georg-August-Universität Göttingen
}

vorgelegt von

Matthias Heim

geboren in Eisenach

Göttingen, 2011 
1. Gutachter: Prof. Dr. med. Dr. rer. nat. Andree Niklas

2. Gutachter: Prof. Dr. med. Manfred E. Heim

3. Gutachter: Prof. Dr. Arndt Krüger

Tag der mündlichen Prüfung: 


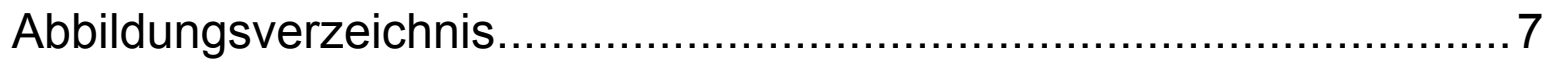

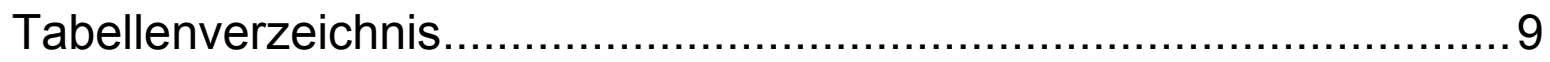

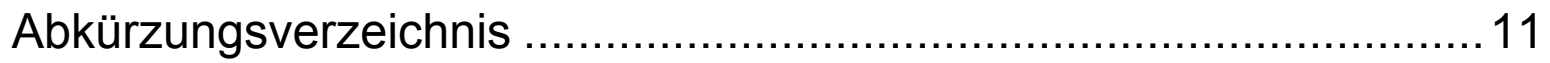

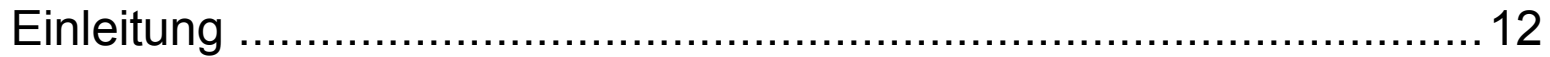

Allgemeiner Teil.................................................................

1. Tumorbedingte Fatigue …..................................................... 14

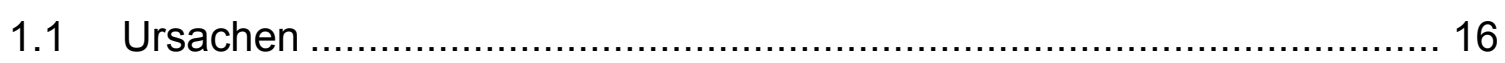

1.2 Auswirkungen tumorbedingter Fatigue auf die Leistungsfähigkeit........... 17

1.3 Therapiemöglichkeiten und Rehabilitation tumorbedingter Fatigue ......... 19

2. Die onkologische Rehabilitation ........................................21

2.1 Patientenkollektiv in der onkologischen Rehabilitation .......................... 21

2.2 Training in der onkologischen Rehabilitation ...................................... 23

3. Krafttraining in der onkologischen Rehabilitation ......................26

3.1 Kraft und Kraftfähigkeit: Begriffsbestimmung...................................... 26

3.2 Arbeitsweisen der Muskulatur und ihre Relevanz für die ..........................

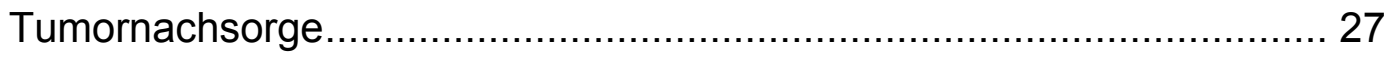

3.3 Kraftarten und deren Relevanz für die Tumornachsorge ...................... 28

3.4 Schlussfolgerungen für das Training in der onkologischen Rehabilitation 28

3.5 Wirkungen eines funktionellen Kraftausdauertrainings ........................... 29

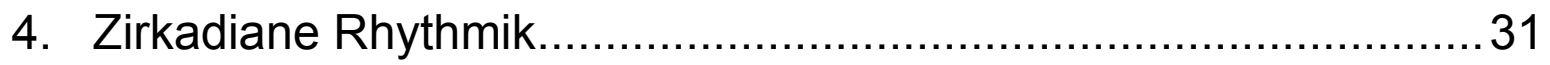

4.1 Einflussfaktoren zirkadianrhythmischer Prozesse auf Training............... 32

4.2 Schlaf und Regeneration im zirkadianrhythmischen Kontext.................. 34

4.3 Zusammenhang zirkadianrhythmischer Parameter und tumorassoziierter Fatigue - Rückschlüsse für das Training onkologischer Patienten .......... 35

Empirischer Teil.............................................................37

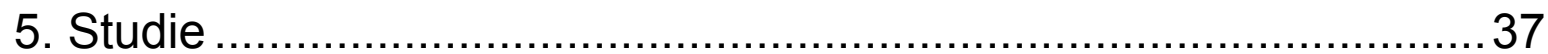

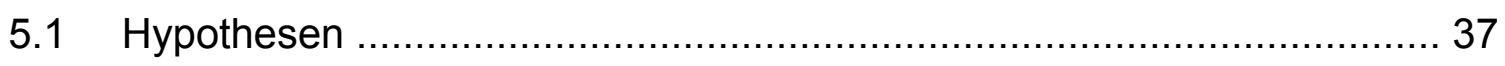

5.2 Aufbau und Inhalt des Trainingsprogramms ..................................... 38

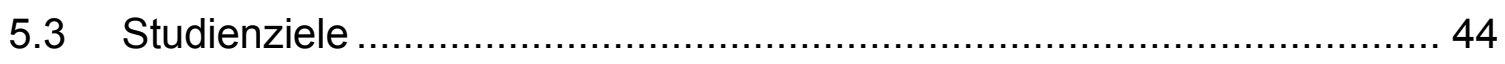

5.4 Patientenrekrutierung und Dauer der Studie ..................................... 45

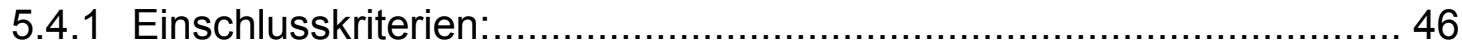

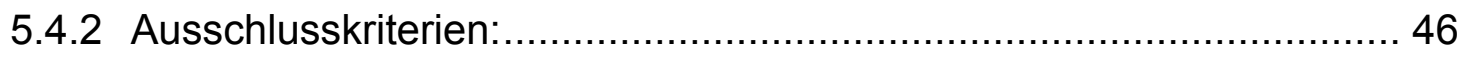




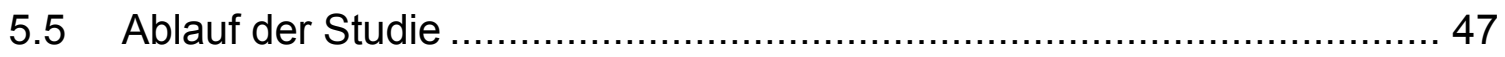

5.6 Material und Methoden .............................................................. 50

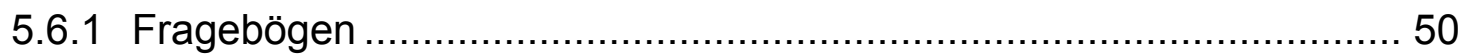

5.6.1.1 Fatigue-LASA (Linear-Analog-Self-Assessment-Scale) ............. 50

5.6.1.2 Screeningbogen zur Fatiguerhythmik................................. 51

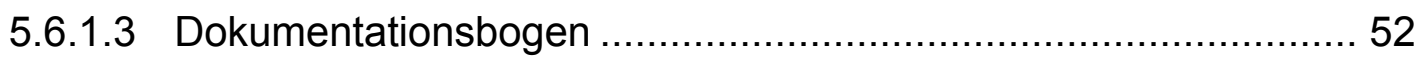

5.6.1.4 HADS-D (Hospital Anxiety and Depression Scale) ................... 53

5.6.1.5 MFI (Multidimensional Fatigue Inventory) ............................... 53

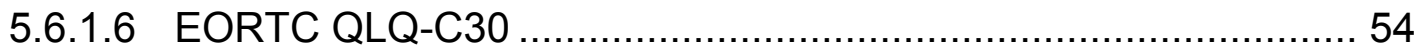

5.6.1.7 FACIT-F (Functional Assessment of Chronic Illness Therapy -

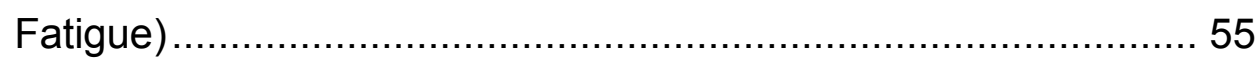

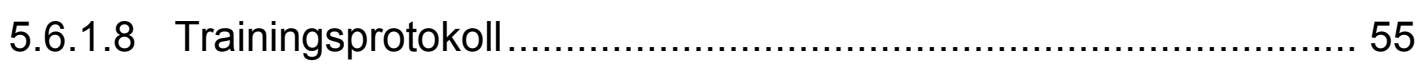

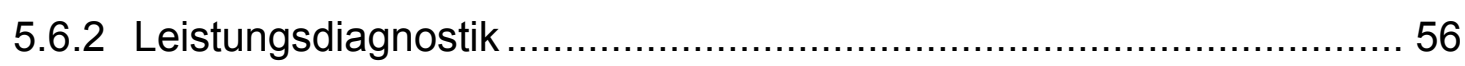

5.6.2.1 Physical Working Capacity (PWC) Test ............................... 56

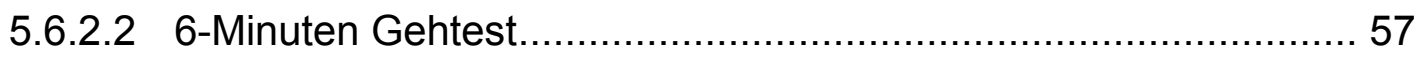

5.6.2.3 Krafttest........................................................................ 58

5.7 Statistische Auswertung der Ergebnisse ..................................... 61

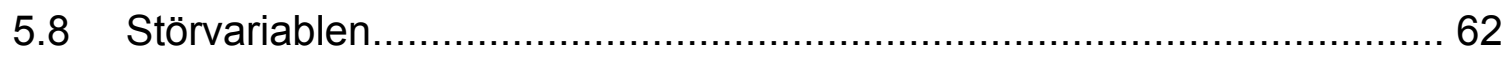

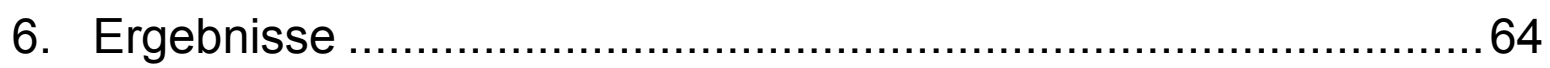

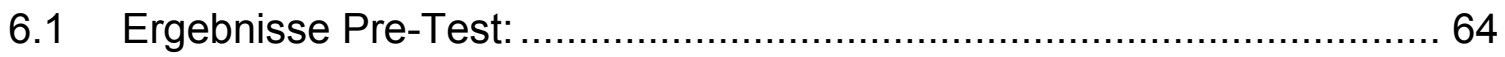

6.1.1 Beschreibung der Pre-Test Stichprobe ..................................... 64

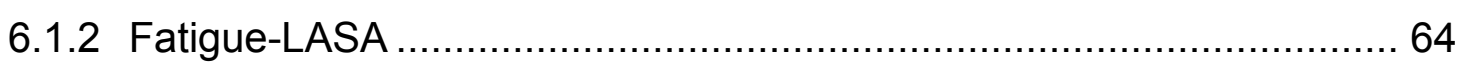

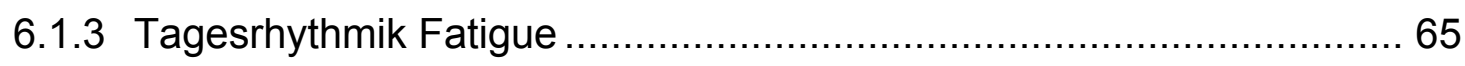

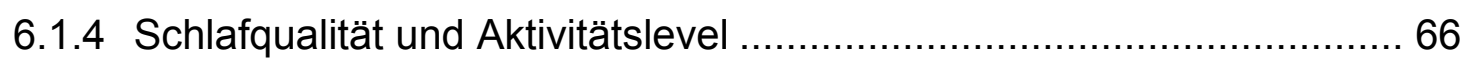

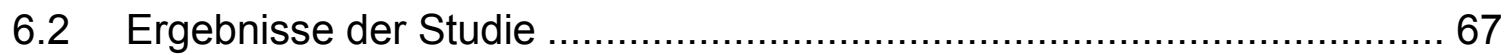

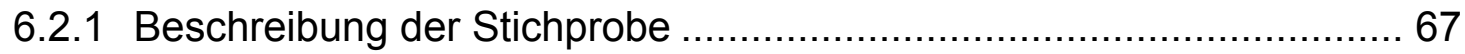

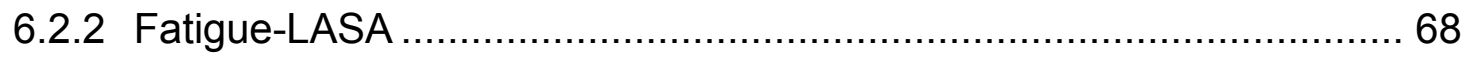

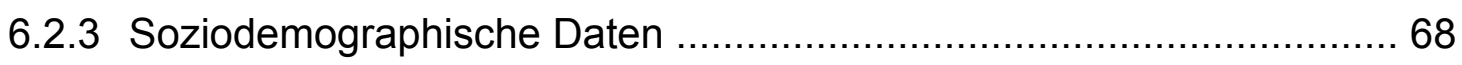

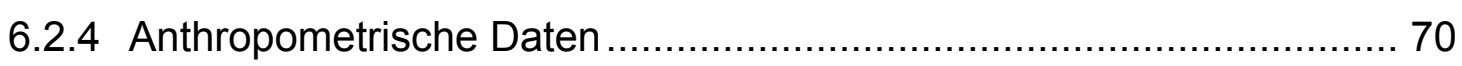

6.2.5 Allgemeine Daten zur Erkrankung ........................................... 72

6.2.6 Allgemeine Daten zu sportlichen Vorerfahrungen ......................... 76

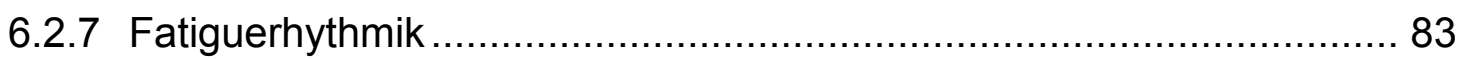

6.2.7.1 Gruppeninterner Vergleich zu allen drei Messzeitpunkten .......... 83

6.2.7.2 Vergleich Kontroll- und Trainingsgruppe zum Messzeitpunkt T1 . 86 
6.2.7.3 Vergleich Kontroll- und Trainingsgruppe zum Messzeitpunkt T2 . 87

6.2.7.4 Vergleich Kontroll- und Trainingsgruppe zum Messzeitpunkt T3 . 88

6.2.8 Schlaf und Aktivitätslevel ...................................................... 89

6.2.9 Ergebnisse Angst und Depression (HADS) ................................. 91

6.2.10 Ergebnisse Multidimensional Fatigue Inventory (MFI) ..................... 93

6.2.11 Ergebnisse European Organisation of Research and Treatment Cancer

$($ EORTC QLQ-C30) ....................................................... 98

6.2.12 Ergebnisse Functional Assessment of Cancer Therapy-Fatigue Scale 106

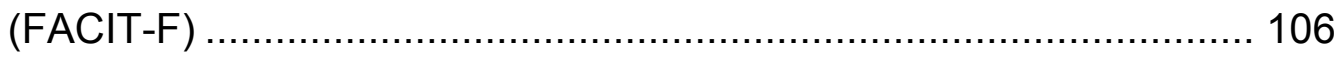

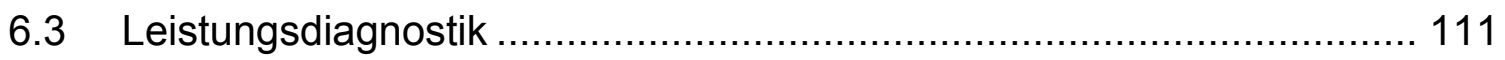

6.3.1 Ergebnisse Ausdauerdiagnostik ........................................... 111

6.3.1.1 6-Minuten Gehtest................................................... 111

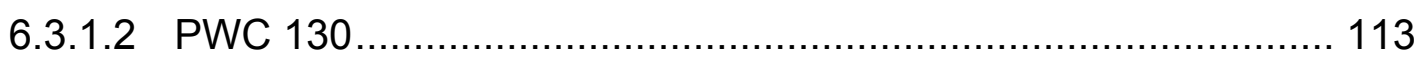

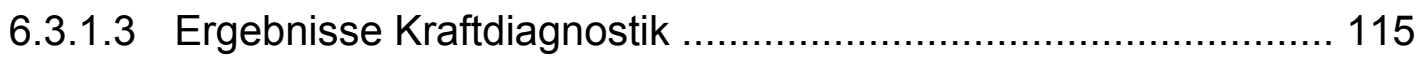

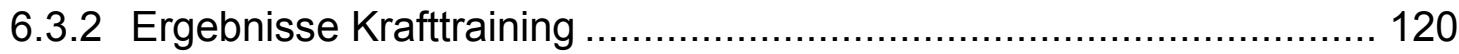

6.3.2.1 Einfluss des tatsächlichen Trainingsumfangs auf verschiedene Untersuchungsparameter.

6.3.2.2 Auswirkungen des tatsächlichen Trainingsumfangs auf den Fatiguerhythmus

6.3.2.3 Auswirkungen des tatsächlichen Trainingsumfangs auf Schlafqualität und Aktivitätslevel ................................... 128

6.3.2.4 Auswirkungen des tatsächlichen Trainingsumfangs auf Angst und Depression (HADS)

6.3.2.5 Auswirkungen des tatsächlichen Trainingsumfangs auf verschiedene Subskalen des MFI

6.3.2.6 Auswirkungen des tatsächlichen Trainingsumfangs auf verschiedene Subskalen des EORTC QLQ-C30

6.3.2.7 Auswirkungen des tatsächlichen Trainingsumfangs auf verschiedene Subskalen des FACIT-F

7. Diskussion 148

7.1 Diskussion Material und Methoden 148

7.1.1 Probandenkollektiv 148

7.1.2 Messinstrumente 
7.1.3 Diskussion des Übungsprogramms ...................................... 152

7.1.4 Diskussion des Dokumentationsbogens zum Training..................... 154

7.1.5 Diskussion der Subgruppen ................................................ 154

7.2 Diskussion der Ergebnisse ................................................. 155

7.2.1 Diskussion der Pre-Test Ergebnisse ...................................... 155

7.2.2 Diskussion der Fatigue-LASA Ergebnisse .................................. 155

7.2.3 Diskussion der Ergebnisse des Dokumentationsbogens .................. 156

7.2.4 Diskussion von Angst und Depression (HADS) ........................... 158

7.2.5 Diskussion der Fatigue (MFI, EORTC) ................................... 159

7.2.6 Diskussion der Lebensqualität (EORTC, FACIT) ......................... 160

7.2.7 Diskussion der Fatiguerhythmik ........................................... 162

7.3 Diskussion der Leistungsdiagnostik........................................... 164

7.3.1 Diskussion der Ausdauertestergebnisse ..................................... 164

7.3.2 Diskussion der Krafttestergebnisse ......................................... 166

7.4 Diskussion der Auswirkungen des tatsächlichen Trainingsumfangs .auf die verschiedenen Untersuchungsparameter ................................... 168

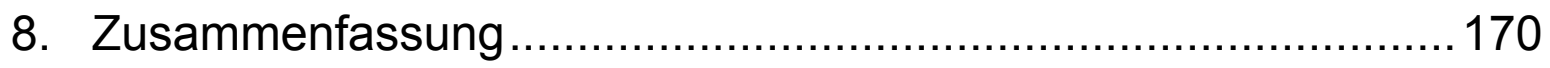

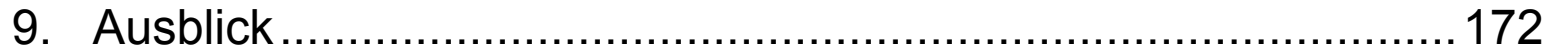

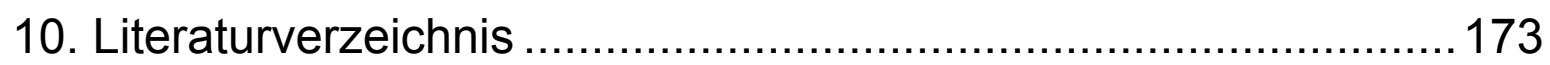

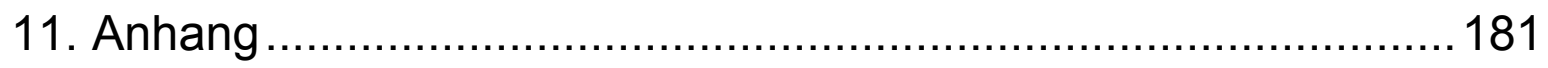




\section{Abbildungsverzeichnis}

Abbildung 1: Patientenbeeinträchtigung (STONE et al. 2000; In: DEUTSCHE KREBSGESELLSCHAFT 2003, Nr. 42, 3)..

Abbildung 2: Teufelskreis: Mangelnde Aktivität und Fatigue

(ELSNER V. D. MALSBURG 2006)

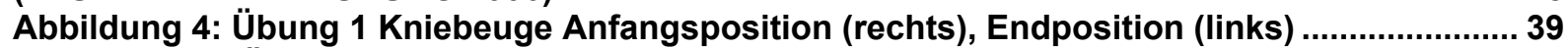

Abbildung 5: Übung 2 Crunch Anfangsposition (rechts), Endposition (links) .......................... 40

Abbildung 6: Übung 3 Sit Up (gerade) Anfangsposition (rechts), Endposition (links) ................ 41

Abbildung 7: Übung 4 Sit Up (schräg) Anfangsposition (rechts), Endposition (links) ...............41

Abbildung 8: Übung 5 Liegestütz Anfangsposition (rechts), Endposition (links) ......................42

Abbildung 9: Übung 6 Latzug Anfangsposition (rechts), Endposition (links) ............................ 42

Abbildung 10: Übung 7 Seitheben Anfangsposition (rechts), Endposition (links) .....................43

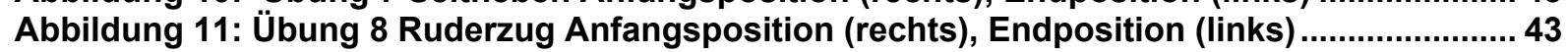

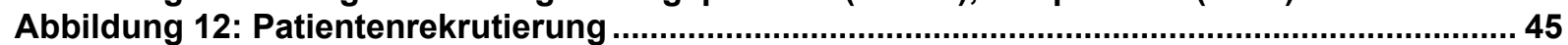

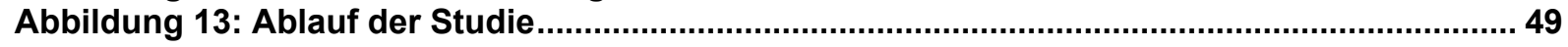

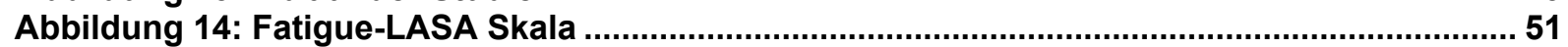

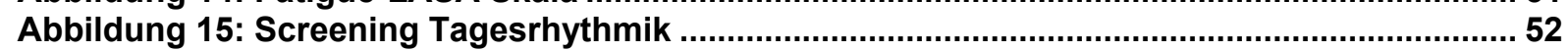

Abbildung 16: Messposition Armbeugung (ELSNER V. D. MALSBURG 2006, 83) .....................59

Abbildung 17: Messposition Kniestreckung (ELSNER v. d. MALSBURG 2006, 82) ...................6 60

Abbildung 18: Tagesrhythmik Fatigue Pre-Test......................................................................6 65

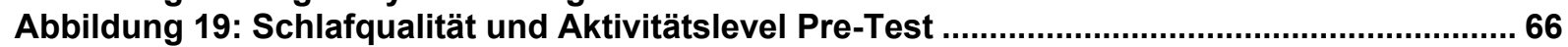

Abbildung 20: Fatigue-LASA Verteilung ............................................................................6. 68

Abbildung 21: Berufliche Situation Kontrollgruppe (links) und Trainingsgruppe (rechts) ......... 70

Abbildung 22: Diagnosen des gesamten Patientenkollektivs ..................................................... 72

Abbildung 23: Diagnosen Kontrollgruppe (links) und Trainingsgruppe (rechts) ....................... 73

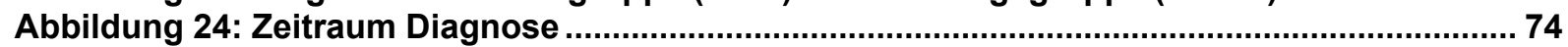

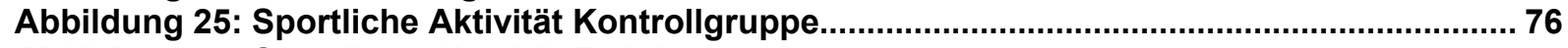

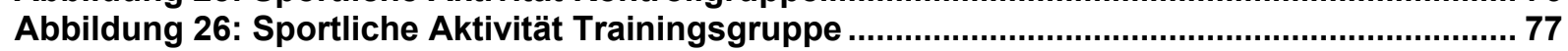

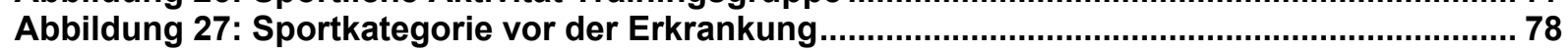

Abbildung 28: Sportkategorie vor der Rehabilitation .......................................................... 79

Abbildung 29: Zeitlicher Aufwand vor Erkrankung (links) und vor Rehabilitation (rechts) ........ 80

Abbildung 30: Sportmotive von Kontrollgruppe (links) und Trainingsgruppe (rechts) .............. 81

Abbildung 31: Subjektive Selbsteinschätzung Kontrollgruppe (links) und Trainingsgruppe

(rechts)...

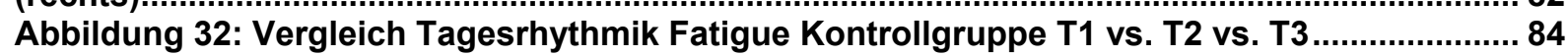

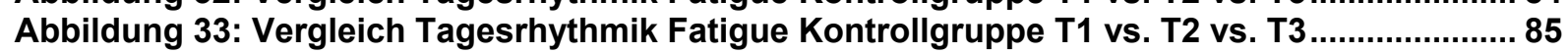

Abbildung 34: Vergleich Tagesrhythmik Fatigue Kontrollgruppe vs. Trainingsgruppe zu T1.... 86

Abbildung 35: Vergleich Tagesrhythmik Fatigue Kontrollgruppe vs. Trainingsgruppe zu T2.... 87

Abbildung 36: Vergleich Tagesrhythmik Fatigue Kontrollgruppe vs. Trainingsgruppe zu T3.... 88

Abbildung 37: Schlafqualität Kontrollgruppe (links) und Trainingsgruppe (rechts)................... 89

Abbildung 38: Aktivitätslevel Kontrollgruppe (links) und Trainingsgruppe (rechts) ..................90

Abbildung 39: HADS Angstscore Kontrollgruppe (links) und Trainingsgruppe (rechts) ........... 91

Abbildung 40: HADS Depressionsscore Kontrollgruppe (links) und Trainingsgruppe (rechts). 92

Abbildung 41: Allgemeine Fatigue Kontrollgruppe (links) und Trainingsgruppe (rechts) .......... 93

Abbildung 42: Physische Fatigue Kontrollgruppe (links) und Trainingsgruppe (rechts) .......... 94

Abbildung 43: Mentale Fatigue Kontrollgruppe (links) und Trainingsgruppe (rechts) ............... 95

Abbildung 44: Reduzierte Aktivität Kontrollgruppe (links) und Trainingsgruppe (rechts)......... 96

Abbildung 45: Reduzierte Motivation Kontrollgruppe (links) und Trainingsgruppe (rechts)..... 97

Abbildung 46: Global Health Kontrollgruppe (links) und Trainingsgruppe (rechts) ....................98

Abbildung 47: Physical Functioning Kontrollgruppe (links) und Trainingsgruppe (rechts) ....... 99

Abbildung 48: Emotional Functioning Kontrollgruppe (links) und Trainingsgruppe (rechts) .. 100

Abbildung 49: Cognitive Functioning Kontrollgruppe (links) und Trainingsgruppe (rechts) ... 101

Abbildung 50: Social Functioning Kontrollgruppe (links) und Trainingsgruppe (rechts) ......... 102

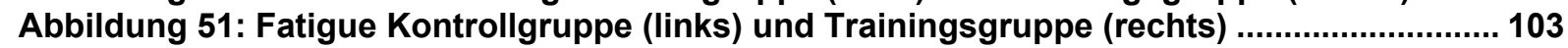

Abbildung 52: Schmerz Kontrollgruppe (links) und Trainingsgruppe (rechts) ........................ 104

Abbildung 53: Schlaflosigkeit Kontrollgruppe (links) und Trainingsgruppe (rechts) ............... 105

Abbildung 54: Körperliches Wohlbefinden Kontrollgruppe (links) und Trainingsgruppe (rechts)

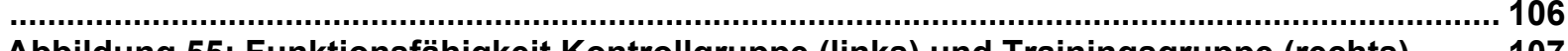

Abbildung 55: Funktionsfähigkeit Kontrollgruppe (links) und Trainingsgruppe (rechts) ........ 107 
Abbildung 56: Seelisches Wohlbefinden Kontrollgruppe (links) und Trainingsgruppe (rechts)

Abbildung 57: Verhältnis zu Freunden und Familie Kontrollgruppe (links) und Trainingsgruppe

(rechts)...

Abbildung 58: Fatiguemodul Kontrollgruppe (links) und Trainingsgruppe (rechts)................. 110

Abbildung 59: 6-Minuten Gehtest Kontrollgruppe und Trainingsgruppe................................... 111

Abbildung 60: PWC 130 Zeitanalyse ................................................................................. 113

Abbildung 61: PWC 130 Stufen Kontrollgruppe (links) und Trainingsgruppe (rechts)............. 114

Abbildung 62: Maximalkraftwerte Arme Kontrollgruppe (links) und Trainingsgruppe (rechts) 115

Abbildung 63: Kraftmittelwerte Arme Kontrollgruppe (links) und Trainingsgruppe (rechts) ... 116

Abbildung 64: Maximalkraftwerte Beine Kontrollgruppe (links) und Trainingsgruppe (rechts)118

Abbildung 65: Kraftmittelwerte Beine Kontrollgruppe (links) und Trainingsgruppe (rechts) ... 119

Abbildung 66: Berechnung optimaler Trainingsumfang .................................................... 120

Abbildung 67: Berechnung des tatsächlichen Trainingsumfangs in Prozent des optimalen

Trainingsumfangs .......................................................................................................... 121

Abbildung 68: Monatsweise Auswertung der einzelnen Übungen ........................................... 122

Abbildung 69: Gesamtauswertung der einzelnen Übungen ............................................... 123

Abbildung 70: Definition der Subgruppen in Prozent des optimalen Trainingsumfangs.......... 124

Abbildung 71: Tatsächlicher Trainingsumfang der drei definierten Subgruppen..................... 125

Abbildung 72: Fatiguerhytmik aller Subgruppen (U-Unterdurchschnitt, D-Durchschnitt, Ü-

Überdurchschnitt) zu den drei Messzeitpunkten .......................................................... 126

Abbildung 73: Fatiguerhythmikvergleich der Untergruppen zum Messzeitpunkt T3................ 127

Abbildung 74: Schlafqualität (links) und Schlafqualität zu T3 (rechts) in Abhängigkeit vom

tatsächlichen Trainingsumfang ...

Abbildung 75: Aktivitätslevel (links) und Aktivitätslevel zu T3 (rechts) in Abhängigkeit vom

tatsächlichen Trainingsumfang

Abbildung 76: Angst zu allen Messzeitpunkten (links) und Angst zu T3 (rechts) in Abhängigkeit vom tatsächlichen Trainingsumfang.

Abbildung 77: Depression zu allen Messzeitpunkten (links) und Depression zu T3 (rechts) in

Abhängigkeit vom tatsächlichen Trainingsumfang ............................................................ 131

Abbildung 78: Allgemeine Fatigue zu allen Messzeitpunkten (links) und Allgemeine Fatigue zu

T3 (rechts) in Abhängigkeit vom tatsächlichen Trainingsumfang ........................................... 132

Abbildung 79: Physische Fatigue zu allen Messzeitpunkten (links) und Physische Fatigue zu T3

(rechts) in Abhängigkeit vom tatsächlichen Trainingsumfang ................................................ 133

Abbildung 80: Mentale Fatigue zu allen Messzeitpunkten (links) und Mentale Fatigue zu T3

(rechts) in Abhängigkeit vom tatsächlichen Trainingsumfang ................................................ 134

Abbildung 81: Reduzierte Aktivität zu allen Messzeitpunkten (links) und Reduzierte Aktivität 135

Abbildung 82: Reduzierte Motivation zu allen Messzeitpunkten (links) und Reduzierte

Motivation zu T3 (rechts) in Abhängigkeit vom tatsächlichen Trainingsumfang ...................... 136

Abbildung 83: Physical Functioning zu allen Messzeitpunkten (links) und Physical Functioning

zu T3 (rechts) in Abhängigkeit vom tatsächlichen Trainingsumfang ...................................... 137

Abbildung 84: Emotional Functioning zu allen Messzeitpunkten (links) und Emotional

Functioning zu T3 (rechts) in Abhängigkeit vom tatsächlichen Trainingsumfang ................... 138

Abbildung 85: Cognitive Functioning zu allen Messzeitpunkten (links) und Cognitive

Functioning zu T3 (rechts) in Abhängigkeit vom tatsächlichen Trainingsumfang ................... 139

Abbildung 86: Global Health zu allen Messzeitpunkten (links) und Global Health zu T3 (rechts)

in Abhängigkeit vom tatsächlichen Trainingsumfang..

Abbildung 87: Fatigue zu allen Messzeitpunkten (links) und Fatigue zu T3 (rechts) in

Abhängigkeit vom tatsächlichen Trainingsumfang

Abbildung 88: Schmerz zu allen Messzeitpunkten (links) und Schmerz zu T3 (rechts) in

Abhängigkeit vom tatsächlichen Trainingsumfang.

Abbildung 89: Schlaflosigkeit zu allen Messzeitpunkten (links) und Schlaflosigkeit zu T3

(rechts) in Abhängigkeit vom tatsächlichen Trainingsumfang

Abbildung 90: Körperliches Wohlbefinden zu allen Messzeitpunkten (links) und Körperliches

Wohlbefinden zu T3 (rechts) in Abhängigkeit vom tatsächlichen Trainingsumfang ................ 144

Abbildung 91: Funktionsfähigkeit zu allen Messzeitpunkten (links) und Funktionsfähigkeit zu

T3 (rechts) in Abhängigkeit vom tatsächlichen Trainingsumfang ......................................... 145

Abbildung 92: Seelisches Wohlbefinden zu allen Messzeitpunkten (links) und Seelisches

Wohlbefinden zu T3 (rechts) in Abhängigkeit vom tatsächlichen Trainingsumfang .

Abbildung 93: Fatigue zu allen Messzeitpunkten (links) und Fatigue zu T3 (rechts) in

Abhängigkeit vom tatsächlichen Trainingsumfang 


\section{Tabellenverzeichnis}

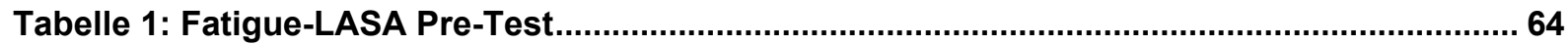

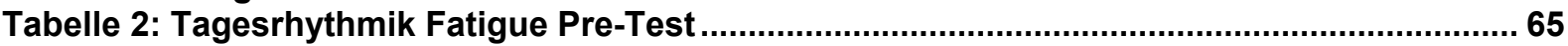

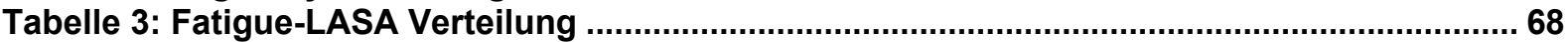

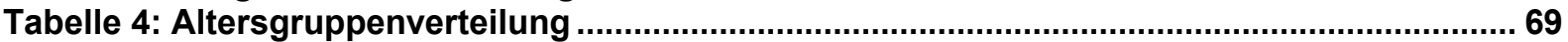

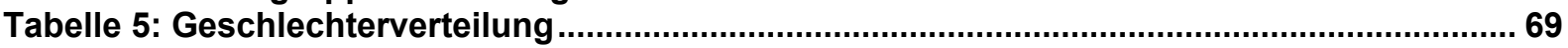

Tabelle 6: Klassifikation BMI im Probandenkollektiv ............................................................ 71

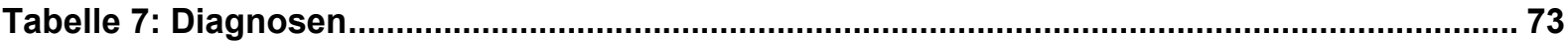

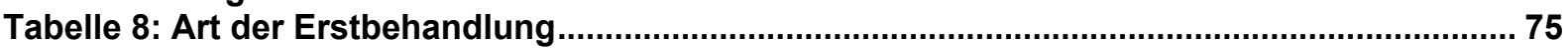

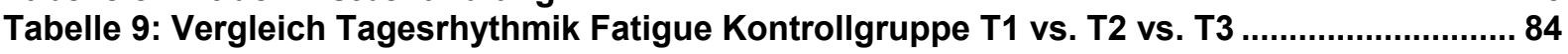

Tabelle 10: Vergleich Tagesrhythmik Fatigue Kontrollgruppe T1 vs. T2 vs. T3 ......................... 85

Tabelle 11: Vergleich Tagesrhythmik Fatigue Kontrollgruppe vs. Trainingsgruppe zu T1 ......... 86

Tabelle 12: Vergleich Tagesrhythmik Fatigue Kontrollgruppe vs. Trainingsgruppe zu T2 ......... 87

Tabelle 13: Vergleich Tagesrhythmik Fatigue Kontrollgruppe vs. Trainingsgruppe zu T3 ......... 88

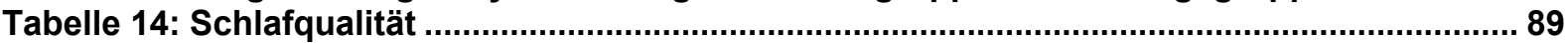

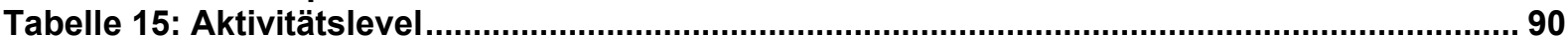

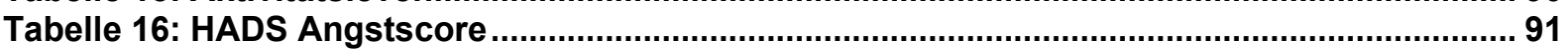

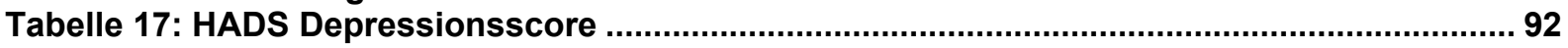

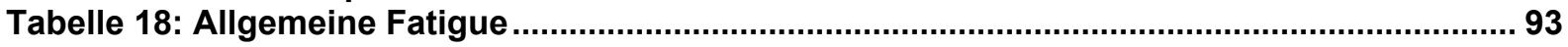

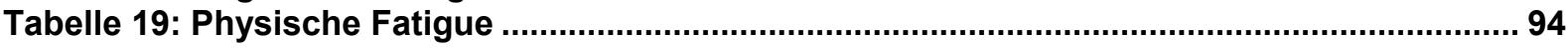

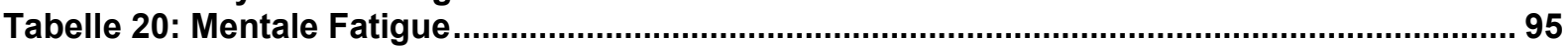

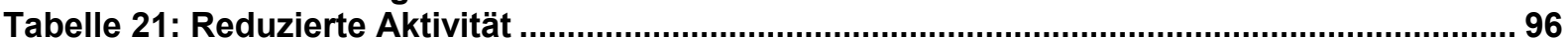

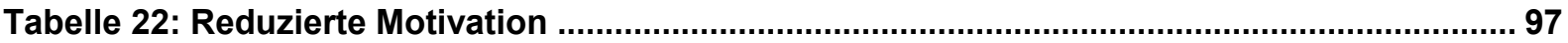

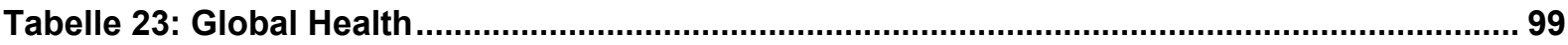

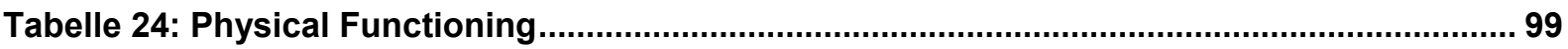

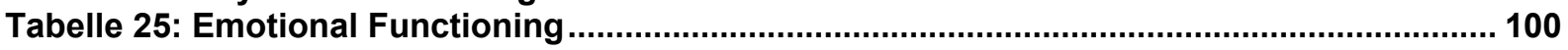

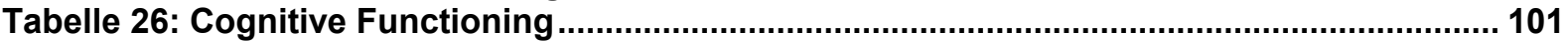

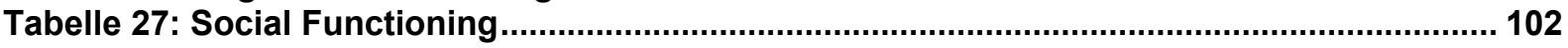

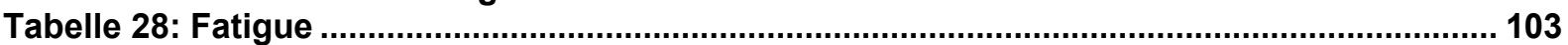

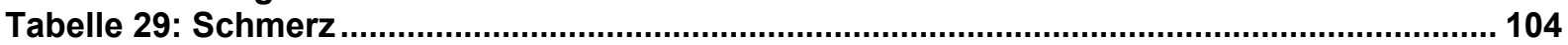

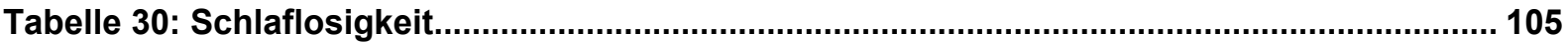

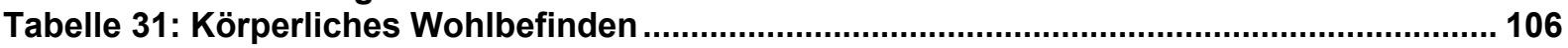

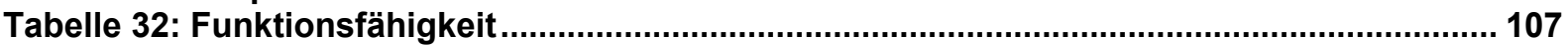

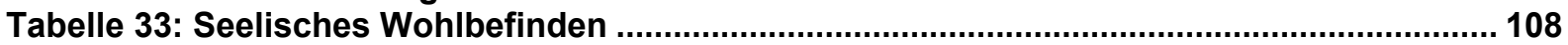

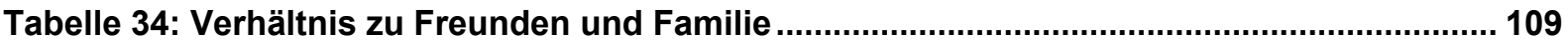

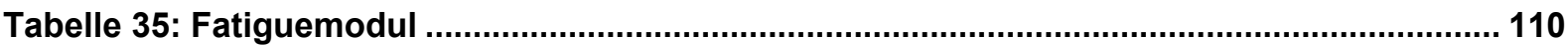

Tabelle 36: 6-Minuten Gehtest Kontrollgruppe und Trainingsgruppe ...................................112

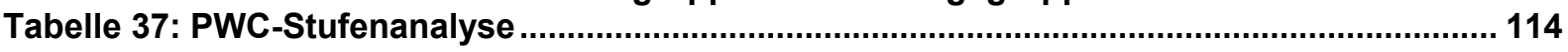

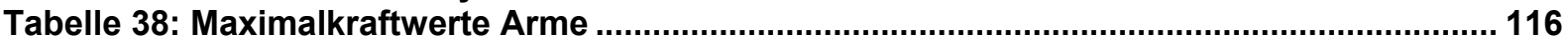

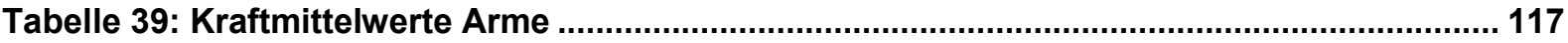

Tabelle 40: Verteilung der Verbesserung bzw. Verschlechterung der maximalen Armkraft..... 117

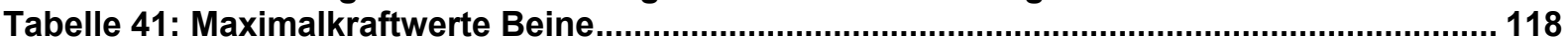

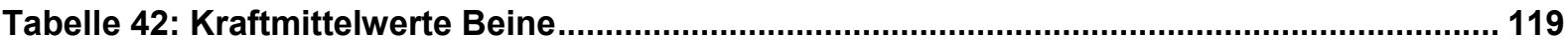

Tabelle 43: Verteilung der Verbesserung bzw. Verschlechterung der maximalen Beinkraft .... 120

Tabelle 44: Optimaler Trainingsumfang pro Trainingseinheit laut Übungsmanual ................... 121

Tabelle 45: Monatsweise Auswertung der einzelnen Übungen.................................................. 122

Tabelle 46: Gesamtauswertung der einzelnen Übungen......................................................... 123

Tabelle 47: Tatsächlicher Trainingsumfang der drei definierten Subgruppen ............................ 125

Tabelle 48: Fatiguerhythmik der Subgruppen zu den drei Messzeitpunkten............................ 126

Tabelle 49: Fatiguerhythmikvergleich der Untergruppen zum Messzeitpunkt T3 ..................... 127

Tabelle 50: Schlafqualität in Abhängigkeit vom tatsächlichen Trainingsumfang ....................... 128

Tabelle 51: Aktivitätslevel in Abhängigkeit vom tatsächlichen Trainingsumfang......................129

Tabelle 52: Angst in Abhängigkeit vom tatsächlichen Trainingsumfang................................130

Tabelle 53: Depression in Abhängigkeit vom tatsächlichen Trainingsumfang .........................131

Tabelle 54: Allgemeine Fatigue in Abhängigkeit vom tatsächlichen Trainingsumfang.............. 132

Tabelle 55: Physische Fatigue in Abhängigkeit vom tatsächlichen Trainingsumfang ............... 133

Tabelle 56: Mentale Fatigue in Abhängigkeit vom tatsächlichen Trainingsumfang................... 134

Tabelle 57: Reduzierte Aktivität in Abhängigkeit vom tatsächlichen Trainingsumfang ............. 135 
Tabelle 58: Reduzierte Motivation in Abhängigkeit vom tatsächlichen Trainingsumfang 136

Tabelle 59: Physical Functioning in Abhängigkeit vom tatsächlichen Trainingsumfang .......... 137

Tabelle 60: Emotional Functioning in Abhängigkeit vom tatsächlichen Trainingsumfang....... 138

Tabelle 61: Emotional Functioning in Abhängigkeit vom tatsächlichen Trainingsumfang....... 139

Tabelle 62: Global Health in Abhängigkeit vom tatsächlichen Trainingsumfang....................... 140

Tabelle 63: Fatigue in Abhängigkeit vom tatsächlichen Trainingsumfang ............................... 141

Tabelle 64: Schmerz in Abhängigkeit vom tatsächlichen Trainingsumfang ............................ 142

Tabelle 65: Schlaflosigkeit in Abhängigkeit vom tatsächlichen Trainingsumfang ....................143

Tabelle 66: Körperliches Wohlbefinden in Abhängigkeit vom tatsächlichen Trainingsumfang144

Tabelle 67: Funktionsfähigkeit in Abhängigkeit vom tatsächlichen Trainingsumfang............. 145

Tabelle 68: Seelisches Wohlbefinden in Abhängigkeit vom tatsächlichen Trainingsumfang .. 146

Tabelle 69: Fatigue in Abhängigkeit vom tatsächlichen Trainingsumfang ................................. 147 


\section{Abkürzungsverzeichnis}

BMI

Body Mass Index

LASA

Linear-Analog-Self-Assessment Scale

FACIT

Functional Assessment of Chronic Illness Therapy

Measurement System

FACIT-F Functional Assessment of Cancer Therapy-Fatigue Scale

HADS

Hospital Anxiety and Depression Scale

MFI

Multidimensional Fatigue Inventory

EORTC

European Organisation for Research and Treatment of Cancer

MEAN

Mittelwert

$\mathrm{M}$.

Musculus

Mal.

Maligne

PWC

Physical Working Capacity

STDEV

Standardabweichung

Abb.

Abbildung

Tab.

Tabelle

u. a.

unter anderem

z. B.

zum Beispiel

usw.

und so weiter

vgl.

vergleiche 


\section{Einleitung}

Tumorbedingte Fatigue ist durch einen konstant hohen Anteil von Betroffenen nach wie vor ein aktuelles Thema. Durch den stark einschränkenden Charakter im alltäglichen Leben stellt tumorbedingte Fatigue für viele Patienten eine der unangenehmsten und langwierigsten Nebenwirkungen von Tumorerkrankungen dar. Zum aktuellen Zeitpunkt steht die Forschung noch immer am Anfang. Zahlreiche Fragen bezüglich tumorbedingter Fatigue können aktuell noch nicht beantwortet werden. So ist es bisher noch nicht gänzlich schlüssig, welche Faktoren genau Fatigue auslösen. Zudem ist bisher nur unzureichend geklärt, warum sich bei manchen Patienten aus einer Nebenwirkung der Therapie eine chronische Krankheit entwickelt.

In den letzten zwei Jahrzehnten wurden vermehrt Studien angestellt, die vorrangig Möglichkeiten der Linderung bzw. Heilung tumorbedingter Fatigue untersuchten. Eines der Hauptforschungsinteressen galt dabei der sportlichen Aktivität. Es konnte belegt werden, dass sportliche Aktivität in Form von Ausdauer- oder Krafttraining positive Effekte auf Fatiguepatienten in nahezu jedem Stadium des Krankheitsverlaufs hat. Die positive Wirkung von Sport auf Fatigue ist daher unstrittig. Allerdings ist die trainingswissenschaftliche Optimierung der sportlichen Aktivität im Verlauf der Rehabilitation und danach nach wie vor eine große Aufgabe. Verschiedene Fragen zu Trainingsintensität, Trainingsumfang oder auch der optimalen Tageszeit für Training konnten noch nicht beantwortet werden.

In der vorliegenden Arbeit werden verschiedene Zielstellungen verfolgt, die Teile dieser ungeklärten Fragestellungen betreffen. Ein Ziel der der Arbeit war es, die Wirkung eines intensiven rehabilitativen Krafttrainings auf die körperliche Leistungsfähigkeit, Fatigue und andere Lebensqualitätsparameter hin zu überprüfen. Der Untersuchungszeitraum hierzu betrug vier Monate.

Weiterhin wurde versucht einen Tagesrhythmus der Fatigue zu ermitteln, um diesen mit zirkadianrhythmisch ablaufenden physiologischen Vorgängen in Bezug auf Training zu untersuchen. Eigentliches Ziel hierbei war es, Erkenntnisse über eine mögliche optimale Tageszeit für Training in der onkologischen Rehabilitation zu gewinnen.

Zusätzlich wurden weitere Erkenntnisse bezüglich Fatigue und Lebensqualität gesammelt und ausgewertet. 
Die Arbeit ist in zwei Teile gegliedert. Der erste Teil beschäftigt sich mit dem aus der Literatur gewonnenen Grundwissen zu tumorbedingter Fatigue, Trainingswissenschaft und zirkadianer Rhythmik. Mit diesem Teil soll eine wissenschaftliche Basis zum zweiten empirischen Teil gelegt werden. Der empirische Teil ist der zentrale Teil der Arbeit, in dem die eigentliche Studie beschrieben, ausgewertet und diskutiert wird. 


\section{Allgemeiner Teil}

\section{Tumorbedingte Fatigue}

Tumorerschöpfung, oder auch tumorbedingte Fatigue, ist eine Nebenwirkung der Krebserkrankung oder der Krebstherapie. Das National Comprehensive Cancer Network definiert tumorbedingte Fatigue wie folgt:

"Tumorbedingte Fatigue ist eine andauernde vom Patienten empfundene Müdigkeit und Erschöpfung, die von der Krebskrankheit oder als Nebenwirkung der Krebstherapie auftreten kann. Es wird dabei in physische, mentale und emotionale Fatigue unterschieden. Kennzeichnend ist, dass die Patienten ein massiv gesteigertes Ruhebedürfnis haben, welches sich unverhältnismäßig zur vorangegangenen Aktivität verhält und durch Ruhephasen nicht zu beheben ist" (AHLBERG et al. 2003, 640).

Tumorassoziierte Fatigue wurde jahrelang nicht ernst genommen, da sie ein subjektives Symptom und dadurch nicht immer leicht zu diagnostizieren ist. Ihre Bedeutung ist für den Patienten substantiell, wird aber von Nichtbetroffenen oft nicht wahrgenommen (FLECHTNER \& BOTTOMLEY 2003). Stone et al. haben in einer Studie festgestellt, dass tumorbedingte Fatigue für die Patienten noch vor Schmerzen und Übelkeit die unangenehmste Nebenwirkung darstellt. Ergebnisse dieser Studie wurden in der Fachzeitschrift der Deutschen Krebsgesellschaft veröffentlicht (Abb. 1) (STONE et al. 2000).

Aus dieser und anderen Untersuchungen weiß man heute, dass tumorbedingte Fatigue ein multifaktorielles Leiden ist, dass Patienten daran hindern kann, während und nach der Therapie ein normales Sozial- und Arbeitsleben zu führen (BARDWELL \& ANCOLI-ISRAEL 2008). 


\section{Was beeinträchtigt sie am meisten?}

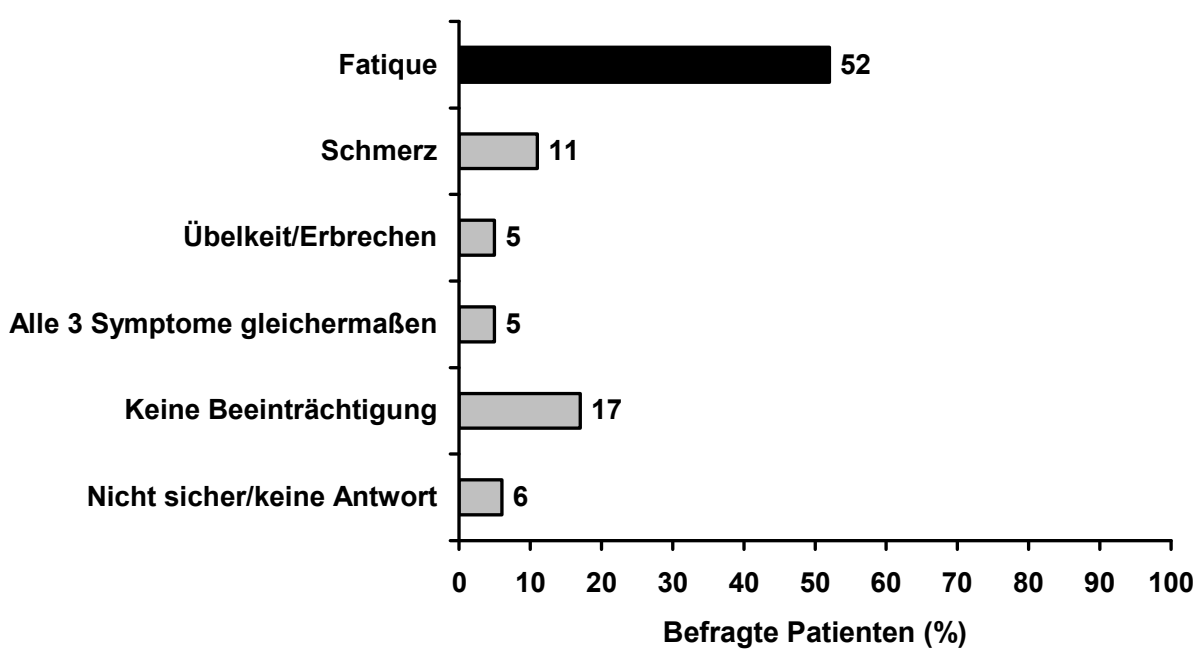

Abbildung 1: Patientenbeeinträchtigung (STONE et al. 2000; In: DEUTSCHE KREBSGESELLSCHAFT 2003, Nr. 42, 3)

Symptome der Tumorerschöpfung sind Müdigkeit, Energiemangel oder Antriebslosigkeit. Hinzu kommen oft auch eine körperliche Schwäche, Konzentrationsstörungen oder Gedächtnisschwierigkeiten. Diese akuten Beschwerden führen dazu, dass die Patienten nicht selten Schwierigkeiten bei der Bewältigung ihres Alltags haben und somit ein geregeltes Arbeits- und Sozialleben unmöglich ist.

Akute Einschränkungen im sozialen Leben haben auch Auswirkungen auf die Lebensqualität. Diverse Studien konnten eine Korrelation zwischen Lebensqualität beeinflussenden Faktoren wie Angst, Depressionen, Schlafstörungen oder Schmerzen und dem Grad der Fatigue feststellen (WAGNER \& CELLA 2004; SERVAES et al. 2001). Eine Linderung tumorbedingter Fatigue sollte demnach eine entscheidende Erhöhung der Lebensqualität nach sich ziehen.

Da tumorbedingte Fatigue eine multifaktorielle Ursachenstruktur aufweist und es durchaus vorkommt, dass keine körperlichen Ursachen vorliegen, wird der Schweregrad durch die subjektive Wahrnehmung des Patienten festgestellt. Als Erhebungsinstrumente dienen Fragebögen zur Selbstbeurteilung. Mittels der Auswertung wird eine Skalierung vorgenommen und der Schweregrad der Fatigue bestimmt. Ein Beispiel für einen Fragebogen zur Erfassung des Schweregrades der Fatigue ist der Fatigue-LASA Bogen. Bei diesem handelt es sich um eine lineare, 
analoge Selbstbeurteilungsskala. Eine genaue Beschreibung dieses Instruments findet sich in Abschnitt 5.5.1.1. Andere Fragebogeninstrumente beinhalten multidimensionale Symptomskalen und ermöglichen so präzisere Aussagen zur vorliegenden Fatigue (JEAN PIERRE et al. 2007). In Abschnitt 5.5.1 werden die in der Studie verwendeten Fragebögen genauer charakterisiert.

Zur Erfassung der Fatigue muss die Krankheit mit allen anfallenden Symptomen beurteilt werden. Daher ist es wichtig, die verschiedene Symptome auch im Zusammenhang und nicht als einzelne, voneinander unabhängige Störungen zu sehen (WEIS et al. 2002)

Maßnahmen der Therapie tumorbedingter Fatigue finden sich in den meisten Therapiekonzepten von Sekundärbehandlungen in Rehabilitationskliniken.

\subsection{Ursachen}

Tumorbedingte Fatigue ist nach heutigen Erkenntnissen eine sehr vielschichtige Krankheit. Obwohl die genauen Ursachen noch nicht geklärt sind, ist man sich bewusst, dass die Gründe, die zur Erkrankung führen, multifaktoriell sind (KREBSGESELLSCHAFT 2003). Darum muss die Ursachensuche sowohl auf körperliche, als auch auf psychisch-soziale Forschung ausgedehnt werden.

Ferner wurde festgestellt, dass bei vielen Patienten die Fatigue erstmals im Zusammenhang mit Primärtherapien auftritt (JACOBSEN et al. 2007).

In einigen Fällen ist ein starker Leistungsknick, als Symptom der Fatigue, das erste Anzeichen für eine Tumorerkrankung überhaupt. Ein Grund dafür kann die hohe Geschwindigkeit sein mit der einige Tumorarten wachsen und dem Körper dadurch große Energiemengen entziehen (KURZROCK 2001). Abwehrreaktionen, wie die vermehrte Sekretion von Interleukinen, Zytokinen, Interferonen oder Tumor-NekroseFaktoren werden ebenfalls als Ursachen diskutiert (ELSNER V. D. MALSBURG, 2006).

Neben den körperlichen Faktoren können auch psycho-soziale Faktoren wie Angst, Depression oder Isolation Fatigue auslösen bzw. verstärken. Die von einer Tumorerkrankung ausgehenden psychischen und emotionalen Belastungen können ursächlich für eine Fatigueerkrankung sein. 
In vielen Fällen erklären sich die Auslöser der Fatigue aus den Nebenwirkungen einiger Primärtherapien. Sowohl Operation, als auch Strahlen- und Chemotherapie können Fatigue verursachen. Es muss in diesem Zusammenhang allerdings zwischen akuter und chronischer Fatigue unterschieden werden. Während akute Fatigue nach einigen Tagen oder Wochen wieder abklingt, bleibt die chronische Form oft noch Monate bis Jahre bestehen.

Hauptursache der akuten Fatigue ist die durch Operation, Chemo-, oder Strahlentherapie ausgelöste Anämie. Durch einen Mangel an roten Blutkörperchen kommt es zu eingeschränktem Sauerstofftransport und dadurch bedingter schneller Ermüdung. Ursachen dieses Mangels liegen in einem Blutverlust oder der negativen Wirkung auf die Produktion von roten Blutkörperchen der Primärtherapien begründet. Bei bis zu 90 \% aller Chemo- und Strahlentherapiepatienten kann durch Schädigung des Knochenmarks oder einem Mangel an dem körpereigenen Wachstumsfaktor Erythropoetin eine Anämie verursacht werden (KREBSGESELLSCHAFT 2003).

Demzufolge ist eine akute Fatigue auch bei fast allen Patienten nach einer dieser Primärtherapien festzustellen. Die Dauer kann dabei sehr variabel sein.

Chronische Fatigue tritt nicht in einer derartigen Häufigkeit auf, hält aber länger an und ist schwieriger zu behandeln (SERVAES et al. 2002). Ursachen dafür liegen, neben den körperlichen Strapazen der Krankheit und Therapie, vorrangig im psychisch-sozialen Bereich.

\subsection{Auswirkungen tumorbedingter Fatigue auf die Leistungsfähigkeit}

„Was genutzt wird entwickelt sich, was nicht genutzt wird verkümmert" (Hippokrates 460 v. Chr. IN: FROBÖSE et al. 2010, 68)

Dieses Zitat beschreibt sehr treffend ein Problem, welches durch Fatigue entstehen kann. Auswirkungen tumorbedingter Fatigue sind hauptsächlich starke Einschränkungen der Lebensqualität durch eine eingeschränkte körperliche Leistungsfähigkeit und durch psychosoziale Probleme.

Körperliche Symptome sind häufig Müdigkeit, Kraftlosigkeit und mangelnde Ausdauer. Symptome im psychosozialen Bereich sind Antriebslosigkeit, Depression, Angst oder auch Gedächtnisschwierigkeiten. 
Die Folgen unbehandelter Fatigue enden nicht selten in einem Teufelskreislauf (Abb. 2). Antriebslosigkeit und mangelnder Bewegung folgen nach einem gewissen Zeitraum auch körperliche Symptome. Kraftlosigkeit, Muskelschmerzen und Kurzatmigkeit, schon bei geringen Belastungen können die Folge von Muskelatrophien sein, die wiederum durch fehlende Belastungen des Muskelsystems entstehen. Nicht selten führen diese zu Beschwerden und erneuter Schonung, da die Patienten sich schon bei geringen Belastungen völlig überfordert fühlen.

Scham, Angst und mangelndes Selbstbewusstsein führen zum Teil zu einer sozialen Isolation und einer Überforderung der Patienten.

Ein Kreislauf entsteht, der sowohl die physischen als auch die psychischen Symptome der Fatigue verschlimmern kann.

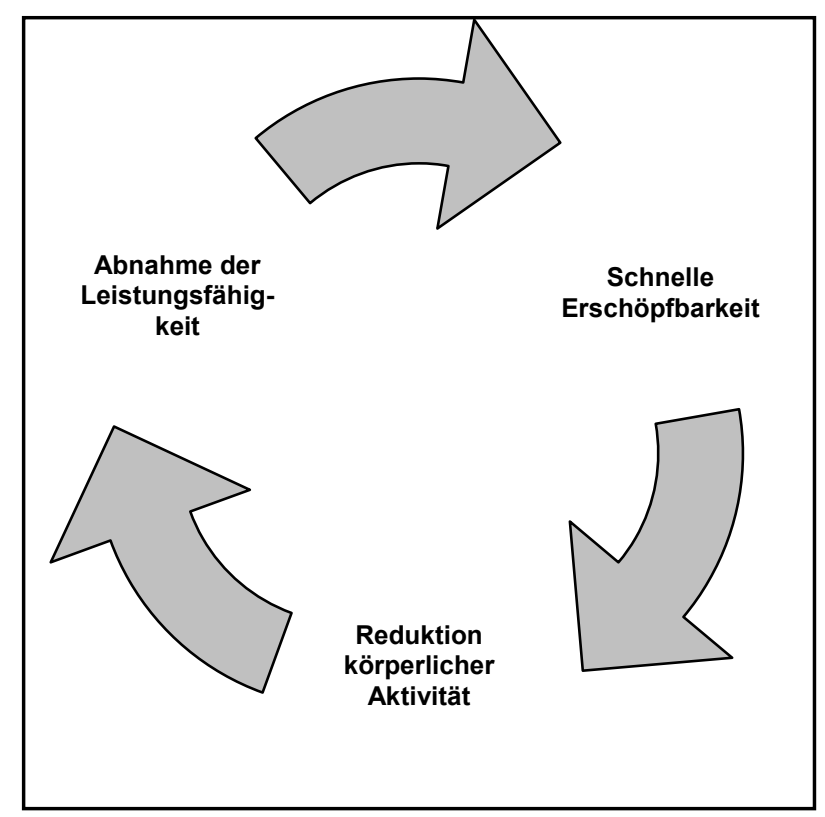

Abbildung 2: Teufelskreis: Mangelnde Aktivität und Fatigue (ELSNER V. D. MALSBURG 2006) 


\subsection{Therapiemöglichkeiten und Rehabilitation tumorbedingter Fatigue}

Aufgrund der multifaktoriellen Ursachenstruktur tumorbedingter Fatigue ist es schwierig, die richtigen Therapieansätze zu finden. Nachdem Fatigue diagnostiziert wurde, sollte eine umfassende ärztliche Untersuchung erfolgen, um mögliche körperliche Ursachen zu identifizieren.

Die schon erwähnte Anämie ist eine der körperlichen Hauptursachen und kann auf verschiedene Arten behandelt werden. Es gibt die Möglichkeit einen kurzfristigen Effekt mittels Bluttransfusion zu erzielen oder durch Verabreichung des Hormons Erythropoetin die Eigenbildung roter Blutkörperchen anzuregen. Diverse Studien konnten zeigen, dass nach einer durch Chemotherapie verursachten Anämie die Transfusionsnotwendigkeit und die Fatigueschwere durch die Verabreichung von Erythropoetin abnahmen und somit eine Verbesserung der Lebensqualität erreicht werden konnte (GABRILOVE et al. 2001; DEMITRI et al. 1998).

Hat tumorbedingte Fatigue überwiegend psychosoziale Ursachen, muss die Therapie an diese angepasst werden. Hierbei haben sich spezielle Therapien ohne pharmakologische Intervention bewährt. Oft werden mit therapeutischer Hilfe Programme erstellt, im Zuge derer situationsbewältigende Maßnahmen entwickelt werden. Inhalte solcher Programme sind sowohl sportliche Aktivität als auch Techniken der Stressbewältigung und Entspannung (ELSNER V. D. MALSBURG 2006).

Ein Faktor, der sich positiv auf körperliche aber auch auf psychische Parameter auswirkt, ist sportliche Aktivität. Zahlreiche Studien belegen eine positive Wirkung körperlicher Aktivität während und nach der Therapie auf die physische und psychische Konstitution onkologischer Patienten (CRAMP \& DANIEL 2008). In der onkologischen Rehabilitation gibt es verschiedene Therapieansätze zur Behandlung tumorassoziierter Fatigue. Als wesentlichstes Ziel rehabilitativer Vorgänge beschreibt Schüle die Wiedereingliederung in soziale und berufliche Strukturen (SCHÜLE 2000). Daraus resultiert ein Höchstmaß an Alltag und Lebensqualität. Ein wesentlicher Aspekt in der Rehabilitationskette ist die stationäre Rehabilitation in einer Sekundärklinik. Die Behandlung in einer solchen Klinik umfasst neben den medizinischen Aspekten onkologischer Erkrankungen, vor allem Maßnahmen der psychosozialen Betreuung, Physiotherapie, Sporttherapie, Ergotherapie und Ernährungstherapie (KLINIKPROSPEKT 2004). Diese Therapieaspekte werden 
unter dem großen Ziel der Wiederherstellung mit unterschiedlicher Gewichtung auf den einzelnen Patienten angewendet. Zusätzlich ist die soziale Interaktion mit anderen Betroffenen ein weiterer wichtiger Faktor.

Aus sportwissenschaftlicher Sicht muss die stationäre onkologische Rehabilitation die Möglichkeit nutzen, den Patienten die Behandlung durch Sport noch näher zu bringen. Dies meint zum einen den Patienten einen Einblick in die Wirkung körperlicher Belastung zu gewähren, um überhaupt ein Verständnis für die Wichtigkeit körperlicher Aktivität zu schaffen. Zum anderen müssen die Patienten trainingswissenschaftlich begleitet werden, um Überforderungen zu vermeiden, eine größtmögliche Wirkung und eine andauernde Motivation zu körperlicher Aktivität zu gewährleisten. Die Patienten müssen Sport als freudvoll, vor allem aber als sinnvolles Mittel begreifen, um mit ihren eigenen Einschränkungen optimal umgehen zu können und ein Höchstmaß an Lebensqualität wiederherzustellen. Zusätzlich müssen Möglichkeiten erschlossen werden, die eine Nachhaltigkeit gewährleisten. Hierzu können heimbasierte Übungsprogramme oder auch Sportgruppen am Wohnort einen Beitrag leisten. 


\section{Die onkologische Rehabilitation}

Der Begriff der Rehabilitation steht für eine Zusammensetzung verschiedener Therapiemaßnahmen mit dem Ziel einer Wiedereingliederung kranker Menschen in soziale und berufliche Strukturen.

Dabei hat die Rehabilitation ein weites Spektrum. Sie reicht über Maßnahmen der Frührehabilitation in der Akutklinik und den Aufenthalt in der fachspezifischen

Rehabilitätionsklinik bis zur medizinischen Nachbetreuung in Rehabilitationssportgruppen am Wohnort. In der Literatur wird von einer Rehabilitationkette gesprochen (SCHÜLE 2000). Sportliche Aktivität und Training sind in jeder Phase der Rehabilitationskette wichtige Bausteine.

\subsection{Patientenkollektiv in der onkologischen Rehabilitation}

Die Patienten in der onkologischen Rehabilitation sind in ihren Charakteristika sehr unterschiedlich. Es lassen sich zahlreiche Unterscheidungspunkte wie Erkrankung, Therapiemethode, Erkrankungszeitpunkt, Alter, Ausprägung der Fatigue usw. finden. Der überwiegende Teil der Patienten hat jedoch mit den gleichen Einschränkungen zu kämpfen. Mangelnde körperliche Leistungsfähigkeit nimmt dabei eine zentrale Rolle ein.

Als wesentliche Ursachen für die eingeschränkte körperliche Leistungsfähigkeit sind in erster Linie Erkrankung und Therapie zu nennen. Schnell wachsende Tumore entziehen dem Körper sehr viel Energie. Operationen können eine zeitweise Immobilität verursachen. Primärtherapien sind oft mit kraftraubenden Nebenwirkungen wie Übelkeit, Durchfall, Anämie usw. verbunden. In diesem Krankheitsstadium scheint körperliche Schonung die einzig logische Konsequenz zu sein. Zusätzlich kann es, bedingt durch die Erkrankung, zu einer schlechten bzw. mangelhaften Ernährung kommen.

Als Folge dieser Krankheits- bzw. Therapiewirkungen schonen sich die Patienten gänzlich. Während an sportliche Aktivität überhaupt nicht mehr gedacht wird, wird 
den Patienten zusätzlich von Angehörigen und zum Teil auch von Ärzten von jeglichen körperlichen Alltagsbelastungen abgeraten.

Aus der fehlenden körperlichen Belastung resultiert eine Abnahme der Muskulatur, die eine Abnahme der Kraft- und Ausdauerleistungsfähigkeit zur Folge hat. Eine dreiwöchige Bettlägerigkeit in Verbindung mit den oben genannten Nebenwirkungen kann eine Muskelatrophie von bis zu 25\% verursachen (PREUSS 2008). Inaktive Muskeln haben im Vergleich zu Gesunden eine bis zu 90\% geringere EMG Aktivität (SCHMIDT \& THEWS 2000). Das bedeutet, dass nicht nur die Muskelmasse abnimmt, sondern auch die nervale Ansteuerung schlechter wird. Die Folge ist, dass nicht mehr alle Muskelfasern bei Bewegungen angesteuert und innerviert werden. Inaktive Muskelfasern atrophieren zusätzlich und die intramuskuläre Koordination verschlechtert sich. Darüber hinaus reagieren inaktive Muskeln verstärkt auf katabole Hormone wie Glukokortikoide. Der Einfluss anaboler Hormone vermindert sich dagegen (HOLLMANN \& STRÜDER 2009).

Leiden die Patienten zusätzlich an einer ausgeprägten Fatigue, kommen sie leicht in den in Abbildung 2 dargestellten Teufelskreis.

Das Alter spielt in der onkologischen Rehabilitation eine nicht unerhebliche Rolle. Mehrheitlich sind die Patienten in der fünften bis sechsten Lebensdekade. Eine Abnahme der Muskelmasse und ein damit verbundener Kraftverlust setzten bereits ab dem 30. Lebensjahr ein. Porter et al. beschreiben ab diesem Zeitpunkt ein Absterben von sechs Prozent der Muskelfasern pro Lebensdekade (PORTER et al. 1995). Darüber hinaus kommt es zu einem Hypotrophieeffekt der Muskelfasern. Resultierend ist ein Kraftverlust bis zum 60. Lebensjahr von 15-20\%. Ursächlich dafür ist eine Reizverarmung, d. h. ein Nichtgebrauch der Muskulatur. Es kommt zu einem Absterben der Alpha Motoneurone des Spinalknotens und einer Degeneration der Axone im Zentralnervensystem (HOLLMANN \& HETTINGER 2000). Zusätzlich steht der Hypotrophieeffekt in engem Zusammenhang mit dem hormonellen System. Anabol wirkende Hormone, wie das Sexualhormon Testosteron, nehmen im Alter ab, was eine Atrophie der Muskulatur begünstigt. Trotz allem steht der Abfall körperlicher Leistungsfähigkeit im Alter mit einer erhöhten Inaktivität und mangelndem Training in Verbindung. Durch adäquates Training könnten viele der oben genannten Prozesse verlangsamt werden. 
Die physiologischen Folgeeffekte von Inaktivität und Alter spielen im Bezug auf die körperliche Leistungsfähigkeit bei onkologischen Patienten zusätzlich eine große Rolle.

Dauerhaft eingeschränkte körperliche Leistungsfähigkeit hat auch psychische Auswirkungen. Mangelndes Selbstbewusstsein, Angst oder Depressionen können durch körperliche Inaktivität noch verstärkt werden. Patienten, die aufgrund der Tumorerkrankung ohnehin unter enormen psychischen und emotionalen Belastungen leiden, können so wieder in einen Teufelskreislauf geraten.

\subsection{Training in der onkologischen Rehabilitation}

Ausgehend von dem Begriff Training sollen im folgenden Abschnitt die sportspezifischen Hauptanforderungen sowie die trainingswissenschaftlichen Prinzipien einer stationären Rehabilitation charakterisiert werden. Ausdauer und Kraft als konditionelle Fähigkeiten sowie Koordination stellen dabei die zentralen Themen dar. Diese sollen trainingswissenschaftlich unter dem Aspekt der Funktionalität aufbereitet und auf die onkologische Rehabilitation angewendet werden.

„Sportliches Training ist das planmäßige wiederholte Ausführen von Bewegungsabläufen mit dem Ziel, die körperliche Leistungsfähigkeit zu steigern oder über längere Zeit zu erhalten“ (MARKWORTH 2006, 63).

„Sportliches Training definiert sich als ein komplexer Handlungsprozess mit dem Ziel der planmäßigen und sachorientierten Entwicklung sportlicher Leistungsfähigkeit" (WEINECK 2010, 21).

Körperliche oder auch sportliche Leistungsfähigkeit, wie sie in beiden Definitionen verwendet wird, stellt dabei ein äußerst komplexes Bedingungsgefüge dar, dass von einer Vielzahl äußerer Faktoren abhängig ist. Abbildung 3 macht deutlich, welche Faktoren sportliche Leistungsfähigkeit beeinflussen können. 


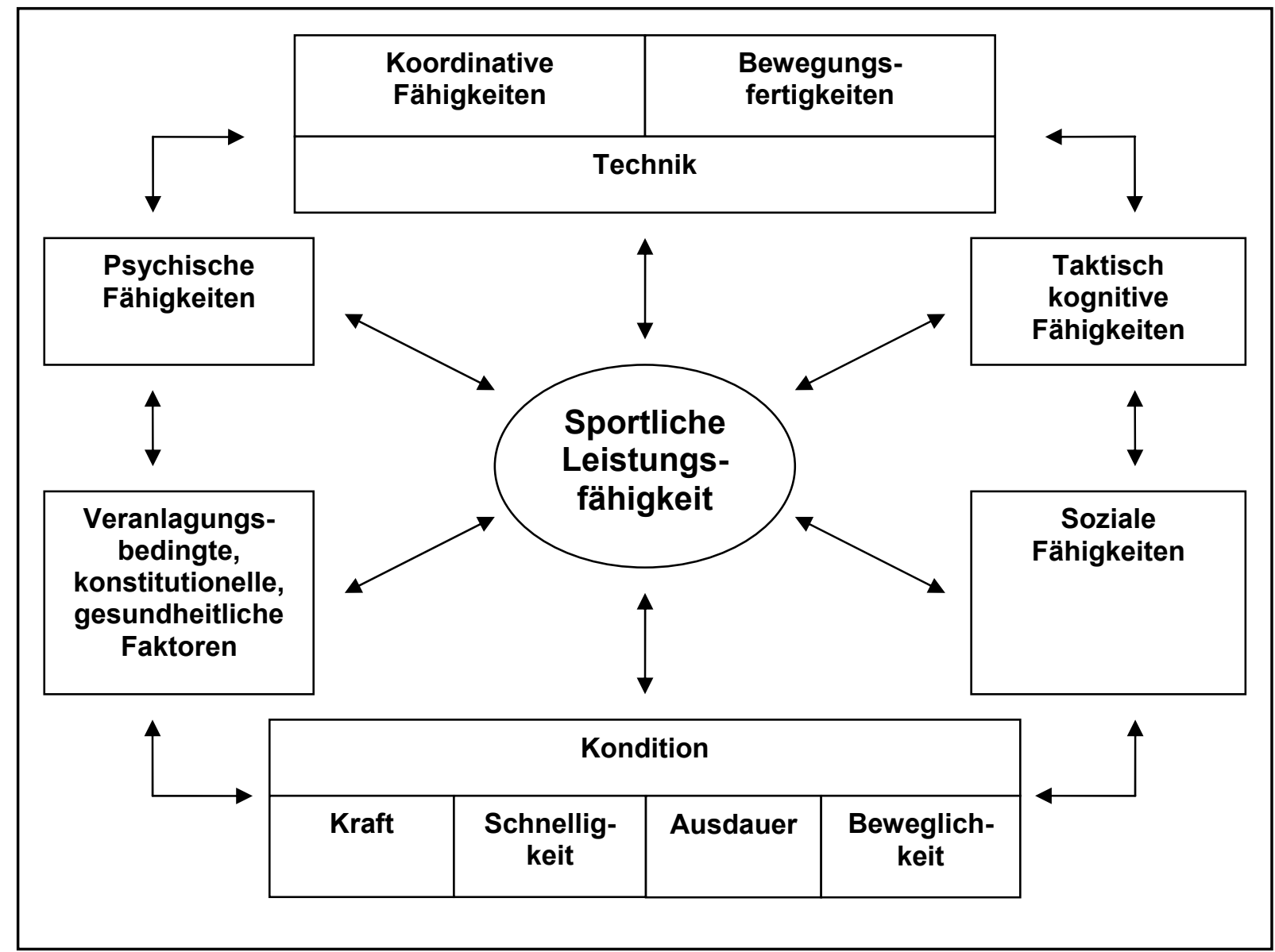

Abbildung 3: Modell der sportlichen Leistungsfähigkeit (WEINECK 2010, 25)

In Anwendung auf onkologische Patienten zielt Training darauf ab, körperliche Leistungsfähigkeit für alltägliche Belastungen wiederherzustellen. Dabei spielt die Wiedereingliederung ins Arbeitsleben eine entscheidende Rolle. Es handelt sich um ein Training der Gesundheitsförderung. Abbildung 3 stellt ein Modell körperlicher Leistungsfähigkeit für den gesunden Menschen dar. In Bezug auf die pathophysiologischen Bedingungen, die in der onkologischen Rehabilitation vorzufinden sind, müssen die Faktoren des Schaubildes neu gewichtet werden. Faktoren wie Taktik und Schnelligkeit erscheinen für das Training onkologischer Patienten irrelevant. Beweglichkeit im Zusammenhang mit konditionellen Fähigkeiten hat eine außerordentlich große Bedeutung für den Leistungssport, in dem eine Optimierung motorischer Bewegung vordergründig ist, um größtmögliche sportliche Leistungen zu erzielen. In diesem Zusammenhang muss auch der Punkt Technik aus der Abbildung genannt werden. Ein hohes Maß an koordinativen Fähigkeiten sowie Bewegungsfertigkeiten sind in allen Sportarten notwendig, um Höchstleistungen zu erbringen. 
Im Gegensatz dazu muss in der onkologischen Rehabilitation aufgrund der veränderten Zielstellung eine Anpassung der in Abbildung 3 aufgezeigten Parameter erfolgen. Natürlich spielt die Beweglichkeit im Zusammenhang mit Kondition auch für onkologische Patienten eine Rolle, allerdings eher im Sinne der Wiederherstellung einer Alltagstauglichkeit. Intra- und intermuskuläre Koordination verbessert sich binnen kurzer Zeit und kann somit schnelle Effekte der Bewegungseffizienz bei Patienten erzielen. Daraus resultiert eine höhere körperliche Leistungsfähigkeit, die wiederum positive Effekte in anderen Bereichen nach sich zieht.

Der pathophysiologische Hintergrund der Zielgruppe verlangt eine angepasste Zielstellung. Im Fokus dieser wäre die körperliche Leistungsfähigkeit sicher besser geeignet als die von Weineck beschriebene sportliche Leistungsfähigkeit.

Um einen optimalen Trainingserfolg mit höchstmöglichem Grad an Anpassungserscheinungen zu erreichen, gilt es verschiedene Trainingsprinzipien zu beachten. Im Folgenden werden einige von Grosser et al. beschriebenen Trainingsprinzipien genannt, die sämtlich auf das Training in der onkologischen Rehabilitation angewendet werden können (GROSSER et al. 2008). Allerdings muss, wie schon erwähnt, die durch den pathologischen Hintergrund veränderte Zielstellung stets beachtet werden.

- Prinzip des wirksamen Belastungsreizes

- Prinzip der progressiven Belastungssteigerung

- Prinzip der Variation der Trainingsbelastung

- Prinzip der optimalen Gestaltung von Belastung und Erholung

- Prinzip der Wiederholung und Dauerhaftigkeit (Kontinuität)

- Prinzip der Individualität und Altersgemäßheit

Die Trainingsprinzipien sind im Anhang unter Anlage 13 erläutert. 


\section{Krafttraining in der onkologischen Rehabilitation}

\subsection{Kraft und Kraftfähigkeit: Begriffsbestimmung}

Kraft oder auch Kraftfähigkeit im Sport ist eine nicht leicht zu erfassende Größe. Während Kraft physikalisch mit der Formel $F$ (Kraft) $=m$ (Masse) * $a$ (Beschleunigung) eindeutig beschrieben ist, stellt eine ausreichende Definition für den Kraftbegriff im Sport eine erhebliche Schwierigkeit dar. Aufgrund der Vielzahl unterschiedlicher Kraftarten sowie der physischen und psychischen Aspekte der Kraft liegen definitorische Schwierigkeiten vor (WEINECK 2010).

Trotzdem soll im Folgenden eine Definition genannt werden, die einen Teil dieser Komplexität in ihrer Formulierung aufzeigt.

„Kraft im Sport ist die Fähigkeit des Nerv-Muskelsystems, durch Innervationsund Stoffwechselprozesse mit Muskelkontraktion Widerstände zu überwinden (konzentrische Arbeit), ihnen entgegenzuwirken (exzentrische Arbeit) bzw. sie zu halten (isometrische Arbeit)" (GROSSER et al. 2008, 40).

Die angesprochenen definitorischen Schwierigkeiten bezüglich der Kraftwirkung umgehen Grosser et al. in ihrer Definition, indem sie einige Arbeitsweisen aufzeigen. Dennoch sind die Vielzahl der Erscheinungsformen sowie die physischen, biomechanischen und psychischen Faktoren nicht genannt.

Im folgenden Abschnitt werden die unterschiedlichen trainingswissenschaftlichen Hintergründe zum Kraftbegriff erläutert und speziell auf die besonderen Aspekte der onkologischen Patienten angewendet. 


\subsection{Arbeitsweisen der Muskulatur und ihre Relevanz für die Tumornachsorge}

Der erste zu beachtende Aspekt ist die Arbeitsweise der Muskulatur. Dabei gilt es zwischen isometrischer, statischer und dynamischer Arbeitsweise zu differenzieren. Bei der dynamischen Arbeitsweise wird in konzentrisch und exzentrisch unterschieden.

Unter statischer Arbeitsweise ist eine verharrende Haltung gegen einen Widerstand zu verstehen. Dabei kommt es unter gleichbleibendem Abstand von Ansatz und Ursprung zu einer Kontraktion im Muskel.

Die konzentrische Arbeitsweise ist durch eine überwindende Muskelarbeit gekennzeichnet, d. h. es wird ein Widerstand durch Muskelarbeit bewegt. Dabei kontrahiert der Muskel und es kommt zu einer Annäherung von Ansatz und Ursprung des Muskels.

Bei der exzentrischen Arbeitsweise handelt es sich um eine nachgebende Muskelarbeit. Ansatz und Ursprung der Muskel entfernen sich bei aktiver Anspannung.

Für ein gesundheitsorientiertes Krafttraining kommt der isometrischen Muskelarbeit die größte Rolle zu, da sie für eine funktionsgerechte Gestaltung der Alltagsmotorik innerhalb des Kraftspektrums maßgeblich ist. Für das Training mit Älteren oder Kranken stellen sich allerdings einige Schwierigkeiten dar. Übende isometrischer Kraftübungen neigen zur Pressatmung. Hierbei erhöht sich der Druck im Brust- und Bauchraum so stark, dass der Rückfluss des Blutes aus den Gliedmaßen und dem Kopf zum Herzen nur eingeschränkt möglich ist, was eine Sauerstoffunterversorgung zur Folge hat (DENK et al. 2003). Außerdem kommt es zu einem erheblichen Blutdruckanstieg. Ab einem Wert von 15\% der isometrischen Maximalkraft kommt es zur Gefäßkompression und somit zu einer Erhöhung des peripheren Widerstands (SCHMIDT \& THEWS, 2000). Zusätzlich können als Folge der Pressatmung Kollapserscheinungen auftreten, die aus der zerebralen Mangeldurchblutung resultieren. Insbesondere die Phase nach dem Pressen scheint in diesem Zusammenhang besonderes Gefahrenpotenzial zu haben, da ein erhöhter Druckabfall auftritt. Dies kann bis zu Herzrhythmusstörungen führen (HOLLMANN \& HETTINGER 2000).

Für ältere und kranke Menschen stellt die Pressatmung ein erhöhtes gesundheitliches Risiko dar und sollte vermieden werden. Isometrische Muskelanspannung eignet sich aufgrund der genannten Risikofaktoren nur bedingt. 
Daher wird die dynamische Arbeitsweise im Rehabilitationstraining bevorzugt angewendet. Exzentrische und konzentrische Bewegungen werden kombiniert eingesetzt, da sie größtmöglichen Erfolg unter geringem Risiko und Nebenwirkungen gewährleisten.

\subsection{Kraftarten und deren Relevanz für die Tumornachsorge}

In der Literatur werden verschiedene Kraftarten unterschieden. Im Wesentlichen unterscheiden alle Autoren in Maximalkraft, Schnellkraft und Kraftausdauer. Weineck ergänzt außerdem noch die Reaktivkraft (WEINECK 2010).

Für ein gesundheitsorientiertes oder rehabilitatives Krafttraining spielen allerdings Maximal-, Schnell- und Reaktivkraft eine eher untergeordnete Rolle. Im Alltag sind Kraftausdauerbelastungen die am häufigsten zu bewältigende Belastung und somit in erster Linie Ziel eines onkologisch rehabilitativen Trainings. Schnabel et al. charakterisieren die Kraftausdauer als eine Beziehung der Kraftfähigkeit und der Ausdauer, die abhängig von der Maximalkraft ist (SCHNABEL et al. 2008). Andere Autoren benutzen ähnliche Definitionen zur Charakterisierung der Kraftausdauerfähigkeit. Eine Verbindung von Kraft- und Ausdauerfähigkeit oder auch Ermüdungswiderstandsfähigkeit ist allerdings überall gleich.

\subsection{Schlussfolgerungen für das Training in der onkologischen Rehabilitation}

Die Patienten sind nach Erkrankung und Therapie oftmals physisch und psychisch geschwächt. Hinzu kommt, dass ein Großteil der Patienten sich in der fünften und sechsten Lebensdekade befindet. Deshalb ist ausschließlich ein gesundheitsorientiertes Krafttraining sinnvoll.

In der Rehabilitation und taucht heute immer wieder der Begriff der Funktionalität oder auch funktionelles Training auf. Das bedeutet, dass ein Training den Funktionen der Muskulatur und des Körpers angepasst sein sollte, um die Aktivitäten des täglichen Lebens zu erleichtern. 
In Anlehnung an die oben getroffenen Aussagen kann davon ausgegangen werden, dass für die onkologische Rehabilitation mit angesprochenem Patientenkollektiv und unter Beachtung der trainingswissenschaftlichen Prinzipien (Anlage 13) ein funktionelles, dynamisches Kraftausdauertraining den größtmöglichen Erfolg bei möglichst geringen Nebenwirkungen bringt.

\subsection{Wirkungen eines funktionellen Kraftausdauertrainings}

Trintscher beschreibt den menschlichen Organismus als Medium, das sich entsprechend einer erhaltenen Information verändert (TRINTSCHER 1967).

Der menschliche Körper kann in Abhängigkeit der Lebens- und Verhaltensweisen extrem unterschiedliche Konstitutionen aufweisen, die sich vor allem in der Funktion äußern. Zimmermann beschreibt den Organismus als offenes, selbstregulierendes und multistabiles System, das in einem genetisch determinierten Raum gewisse Veränderungsspielräume offen lässt. Diese sind verhaltensabhängig, was sich besonders deutlich in den Wirkungen sportlicher Aktivität auf den Organismus äußert (ZIMMERMANN 2000). Beide charakterisieren den menschlichen Organismus als anpassungsfähiges Gefüge, das eine Funktion auch dann gewährleistet, wenn sich exogene Bedingungen ändern.

Ein funktionelles, dynamisches Kraftausdauertraining hat eine positive Wirkung auf den Körper. Speziell mit steigendem Alter und bei chronischen Erkrankungen erscheint die Wichtigkeit immens. Trotzdem sollte angemerkt werden, dass sportliches Training im Allgemeinen weder den Alterungsprozess stoppen kann, noch als Medikament gegen Tumorerkrankungen wirkt. Die positiven Effekte auf körperliche Leistungsfähigkeit, Mobilität und Lebensqualität sind indes unbestritten. Einige positive Effekte von funktionellem, dynamischem Kraftausdauertraining werden im Folgenden aufgeführt (WEINECK 2010; DICKHUTH 2000):

- Verbesserungen der körperlichen Leistungsfähigkeit insbesondere der Kraftfähigkeit

- Verbesserungen der intra- und intermuskulären Koordination

- Positive Veränderung des Hormonhaushalts 
- Verbesserung der psychischen Befindlichkeit

- Erhöhter Energieumsatz

- Prävention verschiedener Erkrankungen

- Erhalt oder Zurückerlangung der Selbstständigkeit

- Positive Wirkung auf das Immunsystem 


\section{Zirkadiane Rhythmik}

Die Zirkadiane Rhythmik ist ein fundamentales Charakteristikum aller physiologischen, biochemischen und psychologischen Funktionen eines Organismus (GROSS 1983). Der Begriff zirkadian leitet sich aus den lateinischen Begriffen circa = ungefähr und dies = Tag ab. Zirkadiane Rhythmen spielen sowohl für Pflanzen als auch für Tiere und den Menschen eine wichtige Rolle. In der Literatur wird in Tagesrhythmen und die eigentlichen zirkadianen Rhythmen unterschieden. Tagesrhythmen werden von den Licht-Dunkel-Zyklen geprägt. Mit diesem liegt einer von vielen externen Oszillatoren vor. Messfühler der Licht-Dunkel-Zyklen ist die Retina (REFINETTI 2000). Externe Zyklen können sowohl von der Umwelt als auch sozial bestimmt sein. Ein Beispiel für einen Umweltzyklus wurde mit dem LichtDunkelzyklus bereits erwähnt. Soziale Zyklen können beispielsweise Arbeitszeiten, soziale Kontakte oder sportliches Training sein.

Zirkadiane Rhythmen werden von inneren Oszillatoren gesteuert. Diese innere Uhr wird auch als zirkadianer Schrittmacher bezeichnet. Es existieren für die verschiedenen zirkadianen Rhythmen des menschlichen Organismus unterschiedliche Schrittmacher. Als einer der zentralen Schrittmacher gilt der Nucleus Suprachismaticus, der im ventralen Hypothalamus liegt (HOLLMANN \& STRÜDER 2009).

Die biologischen Abläufe, die dieser Steuerung unterliegen, reichen von Mitose über Temperaturrhythmen bis hin zu Schlaf-Wachzeiten.

Eine Störung der Kopplung von zyklisch ablaufenden Körperprozessen und Zeitgebern aus der Umwelt führt zur Desynchronisation. Studien belegen, dass zirkadiane Rhythmen in völliger Dunkelheit nach gewisser Zeit völlig desynchronisiert ablaufen (REFINETTI 2000; ARENDT 1993). Ein praktischeres Beispiel aus dem Alltag ist der Jetlag bei längeren Interkontinentalflügen. Das Überspringen mehrerer Zeitzonen und die damit verbundene Desynchronisation des Licht-Dunkel-Zyklus verursacht auch eine Desynchronisation der internen Oszillatoren. Daraus können unter anderem Übelkeit, gastrointestinale Störungen oder Schlafstörungen resultieren. Ray at al. gehen in ihrer Arbeit sogar noch weiter und erklären, dass durch eine Desynchronisation der zirkadianen Rhythmik auch circadian der gesteuerte Hormonspiegel desynchronisiert wird. Die Folgen einer hormonellen 
Desynchronisation können direkte Auswirkungen auf Krebsrisiko und Tumorwachstum haben (RAY et al. 2008).

Für das Training spielen zirkadiane Rhytmen eine ebenso große Rolle. So scheint es sinnvoll Trainingsreize dann zu geben, wenn die internen zirkadianen physiologischen Voraussetzungen gegeben sind. Im folgenden Abschnitt sollen die internen Faktoren zirkadianer Rhythmik in Verbindung mit Training und speziell mit tumorassoziierter Fatigue betrachtet werden. Von größtem Interesse ist es anschließend aus den Parametern der zirkadianen Rhythmik und der tumorassoziierten Fatigue einen möglichen optimalen Trainingszeitpunkt für onkologische Patienten abzuleiten.

\subsection{Einflussfaktoren zirkadianrhythmischer Prozesse auf Training}

Es gibt zahlreiche interne physiologisch zirkadian ablaufende Prozesse, die in verschiedenem Maße Auswirkungen auf Training haben können.

Die Körpertemperatur weist rhythmische Schwankungen auf, die zum Teil durch exogene Faktoren beeinflusst werden. Es wird davon ausgegangen, dass die Regulation der Wärmeproduktion und der Wärmeabgabe durch verschiedene Areale im Hypothalamus erfolgt.

Die Körpertemperatur schwankt beim Menschen mit einer Amplitude von ungefähr $1^{\circ} \mathrm{C}$ und hat einen Tagesmittelwert von circa $37^{\circ} \mathrm{C}$. Das Tagesmaximum erreicht die Körpertemperatur ca. um 18 Uhr. Interessant erscheint, dass der Temperaturzyklus direkt abhängig von dem Licht-Dunkel-Zyklus ist. In der Nacht ist die Körpertemperatur niedriger und steigt in den frühen Morgenstunden vor dem Erwachen wieder an (REFINETTI 2000). Diese physiologische Rhythmik macht absolut Sinn, da höhere Körpertemperaturen die Leistungsfähigkeit begünstigen. Die Leistungsfähigkeit der Muskulatur ist direkt abhängig vom Leistungsniveau auf zellulärer Ebene und diese ist wiederum durch Enzymwirkung bedingt. Für die Enzymwirkung sind höhere Temperaturen vorteilhaft (OSCHÜTZ 1998). Allerdings gilt dies nur in einem bestimmten Bereich. Schwankungen der Körperkerntemperatur von $4{ }^{\circ} \mathrm{C}$ führen bereits zu einer deutlichen Verminderung der Leistungsfähigkeit (DE MARÉES 1979). Andererseits resultiert eben eine erhöhte Körpertemperatur aus körperlicher Aktivität. Die Temperatur im Muskel kann bei erhöhter muskulärer Arbeit 
bis auf $41{ }^{\circ} \mathrm{C}$ ansteigen, ohne dass sich der Trainierende unbehaglich fühlt (ASTRAND et al. 2003). Es wird in diesem Zusammenhang auch von einer Belastungshyperthermie gesprochen. Zudem steigt die Sauerstoffaufnahme und die Durchblutung mit steigender Körpertemperatur, was den zirkadianrhythmisch, thermoregulatorisch optimalen Trainingszeitpunkt am späten Nachmittag erklärt (MOORE-EDE et al. 1982). Hierbei spielen auch exogene Faktoren, z. B. die Umgebungstemperatur eine erhebliche Rolle. Eine Relevanz für körperliche Leistungsfähigkeit im Sport ist also durchaus gegeben. Eine gesteigerte Durchblutung unter höheren Temperaturen wird auch in der Therapie genutzt, um Bestrahlung oder Chemotherapie noch zu effektivieren. Die sogenannte Therapeutische Hyperthermie wird gezielt genutzt, um tumorbetroffene Körperregionen zu erwärmen und anschließend mit Primärtherapie zu behandeln.

Ähnlich wie mit der Körpertemperatur verhält es sich mit Blutdruck, Puls und Atemfrequenz. Auch diese Parameter unterliegen zirkadianrhythmischen Schwankungen, die zeitlich einem ähnlichen Rhythmus folgen wie die Temperatur. Blutdruck, Puls und Atemfrequenz stehen mit der körperlichen Leistungsfähigkeit in engem Zusammenhang. Eine Steigerung von Puls, Blutdruck und Atemfrequenz in den frühen Morgenstunden vor dem Erwachen macht aufgrund der gesteigerten Aktivität und dem daraus resultierenden erhöhten Verbrauch von Sauerstoff durchaus Sinn. Die sympathikusgesteuerten Vorgänge erreichen gegen $18 \mathrm{Uhr}$ ihr Maximum, was wiederum auf eine erhöhte körperliche Leistungsfähigkeit zu dieser Tageszeit schließen lässt.

Auch die meisten Hormone unterliegen zirkadianrhythmischen Schwankungen über den Tag. So lassen sich in der Blutplasmakonzentration in vielen Fällen zu verschiedenen Tageszeiten unterschiedliche hormonelle Konzentrationen nachweisen (MOORE-EDE et al. 1982). Der Blutkortisolspiegel ist beispielsweise am Morgen deutlich höher als am Abend (HOLLMANN \& STRÜDER 2009). Zudem werden einige Hormone episodisch ausgeschüttet. Zirkadian werden die Sekretionsepisoden zu gewissen Zeiten erhöht und in ihrer Intensität gesteigert. Eine endokrine zirkadiane Rhythmik ist nachweisbar und in ihrem Grundrhythmus stabil. Die Änderung exogener Einflussfaktoren kann allerdings auch hier zu einer Phasenverschiebung der zirkadianen Rhythmik führen. Einstellungsänderungen der zirkadianen Rhythmik von Sporttreibenden sind durchaus möglich und können durch Perioden unterschiedlicher Trainingsintensität oder kontinentalen Ortswechsel 
erfolgen (WURSTLER \& KELLER 1985). Unter den pathophysiologischen Aspekten onkologischer Patienten ist eine Einstellungsänderung des hormonellen Systems oft therapiebedingt. In vielen Fällen von Brust-, Gebärmutter- oder Prostatakrebs werden die Patienten über einen längeren Zeitraum mit einer Hormontherapie behandelt, die eine Veränderung des Hormonhaushalts der Sexualhormone bewirkt.

Mit den psychophysiologischen Parametern sei eine letzte Einflussgröße zirkadianer Rhythmik genannt, die wesentlichen Einfluss auf die sportliche Leistungsfähigkeit hat. Nachweislich zeigen sich auch in diesem Bereich zirkadianrhythmische Schwankungen. Ein Beispiel dafür ist das Aktivierungsniveau der Hirnrinde (OSCHÜTZ 1991). Die sensomotorische und koordianative Leistungsfähigkeit unterliegen somit ebenfalls zirkadianrhythmische Schwankungen.

Die Tagesgänge der verschiedenen Funktionen unterscheiden sich in der Amplitude und der tageszeitlichen Lage der Minima und Maxima. Von trainingswissenschaftlichem Interesse ist die Tageszeit, zu der die Konstellation einen optimalen Trainingserfolg verspricht.

Allerdings ist nur zum Teil bekannt, wodurch zirkadianrhythmische Prozesse gestört werden und inwieweit Tumorerkrankungen und tumorassoziierte Fatigue dabei eine Rolle spielen.

\subsection{Schlaf und Regeneration im zirkadianrhythmischen Kontext}

Zu den zirkadianrhythmischen Vorgängen gehören auch die Schlaf-Wachzeiten. Diese richten sich bei den meisten Menschen nach den Licht-Dunkel-Zyklen. Erholsamer Schlaf ist eine der Hauptregenerationsphasen des menschlichen Organismus. Die Bedeutsamkeit für Leistungsfähigkeit und Lebensqualität ist daher unstrittig. In der Literatur wird auch in „Lerchen“ und „Eulen“, also Frühaufsteher und Nachtschwärmer unterschieden (PHILLIPS 2009). Diese Unterscheidung kann für die Leistungsfähigkeit zu bestimmten Zeiten eine Rolle spielen. Während „Lärchen frühmorgens schon fit sind, ist der Höhepunkt der „Eulen eher zu einem späteren Tageszeitpunkt.

Der Wechsel zwischen den verschiedenen Schlafphasen, die beide essentiell sind, hängt mit der zirkadian verlaufenden Temperaturregulation zusammen (SCHMIDT \& THEWS 1995). Schlafstörungen oder Schlafentzug können neben 
Konzentrationsstörungen zunächst nicht zu ernsten gesundheitlichen Auswirkungen führen. Allerdings kann durch starke Schlafstörungen Fatigue ausgelöst werden (RAY et al. 2008). Psychisch können sich Schlafstörungen hingegen sehr negativ auswirken.

Erholungsmechanismen sind für die Wirkung einer erwünschten Trainingsanpassung von absoluter Wichtigkeit. Zusätzlich wirkt sich eine an die Belastung angepasste Ernährung positiv aus. Training und Erholung sollten zirkadianrhythmisch sinnvoll in den Tagesablauf eingeordnet werden. Die Länge der benötigten Erholungsphasen hängt dabei stark von der Gesamtbelastung des Trainings ab (FROBÖSE et al. 2010).

Bei Tumorpatienten mit akuter Fatigueproblematik müssen die pathophysiologischen Aspekte der Erkrankten beachtet werden. In die Planung der Belastungs- und Erholungsphasen müssen diese Aspekte mit einbezogen werden, um die Gefahr von unerwünschten Effekten zu minimieren. Speziell die Gefahr einer Überforderung ist bei Fatiguepatienten gerade zu Beginn sportinduzierter Maßnahmen außerordentlich hoch. Die hiermit verbundenen negativen körperlichen, aber vor allem motivationalen Nebeneffekte wären einem weiteren Training äußerst abgängig.

Die Planung von Trainings- und Regenerationsphasen sollte nach trainingswissenschaftlichen Erkenntnissen erfolgen. Oschütz sieht bevorzugt die Zeiträume zwischen 10 - 12 Uhr und zwischen 16 - 18 Uhr für sportliche Belastungen vor, während die Zeiträume von 13 - 15 Uhr und nach 19 Uhr der Regeneration vorbehalten sind (OSCHÜTZ 1991).

\subsection{Zusammenhang zirkadianrhythmischer Parameter und tumorassoziierter Fatigue - Rückschlüsse für das Training onkologischer Patienten}

Es lassen sich durchaus Zusammenhänge bezüglich tumorassoziierter Fatigue und zirkadianer Rhythmik nachweisen. Ancoli-Israel et al. untersuchten Fatigue, Schlafstörungen und zirkadiane Rhythmik im Vorfeld einer Chemotherapie bei Brustkrebspatientinnen. Sie konnten nachweisen, dass bei einigen Frauen trotz Schlafstörungen und hoher Fatigue die zirkadianrhythmischen Prozesse stabil blieben. Frauen mit desynchronisierter zirkadianer Rhythmik hatten signifikant höhere Funktionsstörungen über den Tag (ANCOLI-ISRAEL et al. 2006). 
In den letzten Abschnitten über zirkadiane Rhythmik ist deutlich geworden, dass sich gewisse Tageszeiten besonders für Training anbieten. Sinz führte bereits 1980 in seiner Arbeit an, dass tageszeitliche Unterschiede im Bezug auf die Leistungsfähigkeit bestehen (SINZ 1980). Er belegte dies mit Reaktions- und Kurzzeitgedächtnistests. Oschütz nennt diverse Studien, die deutliche Vorteile bei einem Training gegen 18 Uhr nachweisen konnten (OSCHÜTZ 1991). Bisher lassen die Ergebnisse tagesverlaufsdiagnostischer Fatigueuntersuchungen darauf schließen, dass um 18 Uhr ein im Vergleich hohes Fatiguelevel vorliegt. Inwieweit ein erhöhtes Fatiguelevel die Effektivität von Training beeinflusst ist noch nicht hinreichend bekannt. Es lassen sich an dieser Stelle nur Vermutungen anstellen. Wenn ein hohes Fatiguelevel die Effektivität von Training begünstigt, würde sich eine Trainingszeit am späten Nachmittag anbieten.

Allerdings gilt zu überlegen, dass es bei einem rehabilitativen Krafttraining von 30 Minuten einer hohen Konzentration bedarf. Somit ist es eher fraglich, ob eine Trainingszeit bei hohem Fatiguelevel sinnvoll wäre.

Eine aus zirkadianrhythmischer Perspektive ebenfalls günstige Trainingszeit wurde im letzten Abschnitt zwischen 10 Uhr und 12 Uhr genannt. Zu dieser Zeit zeigen sich auch niedrigere Fatiguewerte als am späten Nachmittag. Wenn davon ausgegangen wird, dass ein niedriger Fatiguewert sich positiv auf die Effektivität von Training auswirken würde, erschiene die Zeit zwischen $10 \mathrm{Uhr}$ und $12 \mathrm{Uhr}$ sowohl aus der Fatigue- als auch aus der zirkadianrhythmischen Perspektive optimal.

Mit den hier aufgeworfenen Fragen lässt sich indes keine eindeutige Aussage über die Effektivität von Training im Zusammenhang von zirkadianrhythmischen und Fatigueparametern treffen. Untersuchungen zu diesem Thema wären jedoch wünschenswert, könnte der hier beschriebene Zusammenhang doch die Effektivität der onkologischen Rehabilitation steigern. Trainingswissenschaftliche Erkenntnisse aus weiterführenden Studien müssten direkt in der Therapieplanung umgesetzt werden. 


\section{Empirischer Teil}

\section{Studie}

\subsection{Hypothesen}

1. Tumorassoziierte Fatigue ist noch immer eine stark verbreitete Nebenwirkung von Tumorerkrankungen. Ein hoher Anteil von Patienten wird erhebliche Beschwerden durch Fatigue haben.

2. Durch das Sport- und Bewegungsprogramm der Klinik werden in beiden Gruppen Verbesserungen der Kraft und Ausdauer festzustellen sein. Diese werden aufgrund des kurzen Trainingszeitraums größtenteils auf eine Verbesserung der Koordination zurückzuführen sein.

3. Die Messwerte für Lebensqualität und Fatigue sowie für Angst und Depression werden sich während der stationären Rehabilitation bei allen Teilnehmern verbessern. Im Zeitraum nach der stationären Rehabilitation werden die Messwerte in der Kontrollgruppe stagnieren oder sogar absinken. In der Trainingsgruppe werden sich die Werte weiter verbessern.

4. Die Messwerte der Fatigue zu verschiedenen Tageszeitpunkten werden im Tagesverlauf ansteigen.

5. Die Messwerte der Fatiguerhythmik werden sich in beiden Gruppen während der stationären Rehabilitation verbessern. Nach der stationären Rehabilitation werden sich ausschließlich die Werte der Trainingsgruppe weiter verbessern. In der Kontrollgruppe wird dieser Effekt nicht nachweisbar sein.

6. Die Trainingszeit für das rehabilitative Krafttraining muss zu einem Zeitpunkt gewählt werden, zu dem die Patienten eine hohe Konzentrations- und Leistungsfähigkeit haben. Das gewährleistet eine höhere Effektivität. 
7. Die subjektive Selbsteinschätzung der körperlichen Leistungsfähigkeit wird sich während der stationären Rehabilitation in beiden Gruppen verbessern. Nach drei Monaten werden sich die Probanden der Trainingsgruppe weiterhin besser einschätzen. In der Kontrollgruppe wird die Verbesserung stagnieren oder sogar wieder absinken.

\subsection{Aufbau und Inhalt des Trainingsprogramms}

Das Trainingsprogramm ist ein rehabilitatives Krafttraining zur Erhöhung der funktionellen Körperkraft, Koordination und zur Erhaltung und Verbesserung der Mobilität.

Die im Trainingsprogramm beinhalteten acht Übungen wurden in Zusammenarbeit mit der physiotherapeutischen Abteilung der Sonnenberg-Klinik aus einem zuvor zusammengestellten Übungskatalog von 54 Übungen ausgewählt. Auswahlkriterien für die Übungen waren unter anderem Schwierigkeitsgrad, Funktionalität und Durchführbarkeit für den Patienten. Ein weiterer maßgeblicher Faktor war es, mit acht Übungen einen maximalen Effekt zu erzielen. So sollte es gelingen, mit den ausgewählten Übungen die wichtigsten Muskelgruppen des Körpers zu trainieren und somit ein Ganzkörpertraining zu entwickeln. Die Übungen wurden zum Teil mit dem Hilfsmittel Therra-Band ausgeführt.

In dem Übungsmanual für die Patienten waren die Übungen in der Ausgangs- und in der Endposition abgebildet. Zusätzlich wurden stichwortartig Knotenpunkte der Bewegung beschrieben und es gab eine empfohlene Wiederholungszahl.

Im Folgenden werden die einzelnen Übungen vorgestellt. Zusätzlich werden vorrangig arbeitende Muskulatur und deren Arbeitsweise erklärt. Hierbei wird sich bei komplexeren Übungen an eine Erklärungsmethode von Tittel gehalten, in der Bewegungen nicht durch den Einsatz einzelner Muskeln erklärt werden, sondern dadurch, dass sich verschiedene zusammenarbeitende Muskeln in Schlingen oder Ketten vereinen. In diesem Modell wird besonders darauf Wert gelegt, dass erst ein Zusammenspiel der Muskulatur, die intermuskuläre Koordination, Gewähr für einen reibungslosen, ökonomischen und zugleich ästhetischen Bewegungsablauf gibt (TITTEL 1994). Allerdings wird das Modell nicht in voller Komplexität angewendet 
und erklärt, da dies zu umfangreich wäre. Trotzdem erscheint die Betrachtungsweise nach Tittel sinnvoll, da sie eine natürliche Funktionalität in der Zusammenarbeit der Muskulatur bei komplexen Bewegungen unterstreicht.

\section{Kniebeugen}

Kniebeugen sind eine Übung zur Kräftigung großer Teile der Beinmuskulatur, sowie der Rumpfmuskulatur. Außerdem ist durch die Gleichgewichtsanforderung der Übung ein hoher koordinativer Anspruch gegeben.

Die Beinmuskulatur arbeitet exzentrisch bei der Abwärtsbewegung und konzentrisch in der Aufwärtsbewegung. Die Streckschlinge besteht im Wesentlichen aus dem M. gluteus maximus, verläuft über den $M$. quadriceps femoris und dem $M$. gastrocnemius.

Hinzu kommt die isometrisch stabilisierend arbeitende Rumpfmuskulatur und die, durch die Armhaltung innervierte Schultermuskulatur. Hauptsächlich arbeitende Muskeln sind die Bauchmuskeln (M. rectus abdominis, der M. obliquus externus abdominis, der M. obliquus internus abdominis) und der lange Rückenstrecker (M. longissimus dorsi). Mit einer kontrollierten Ausführung der Bewegung kann zusätzlich eine Kräftigung kleinerer haltungsstabilisierender Hilfsmuskeln erreicht werden. Zusätzliche Zielstellung dieser Übung ist daher auch die Verbesserung der Körperhaltung und der -wahrnehmung.

Die zur Schulterhaltung beanspruchten Muskeln sind hauptsächlich der M. trapezius und der M. deltoideus (Pars clavicularis deltoidei).
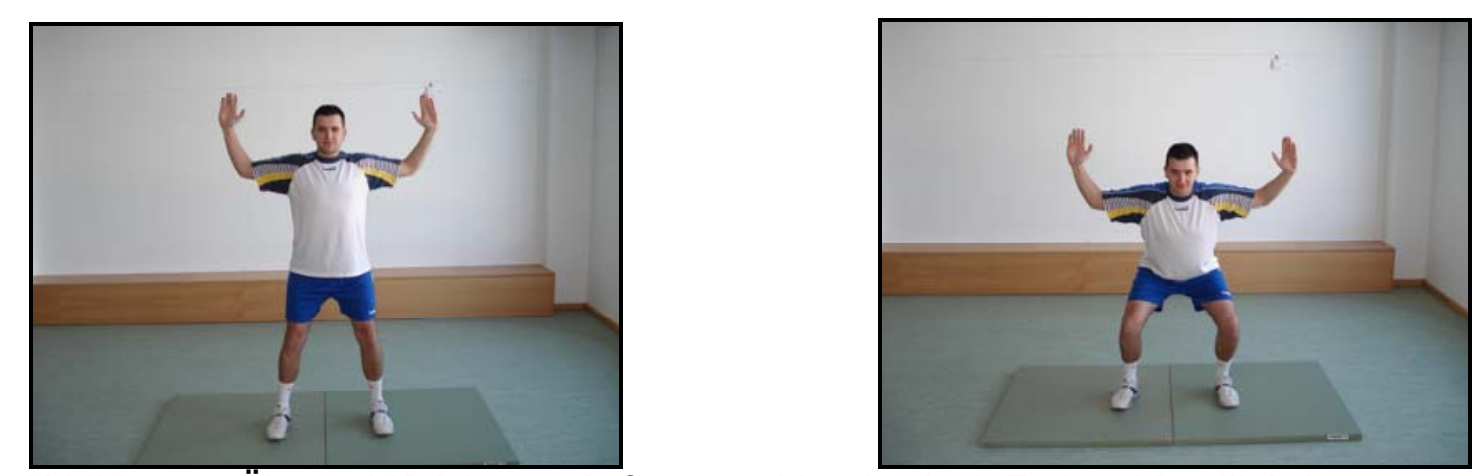

Abbildung 4: Übung 1 Kniebeuge Anfangsposition (rechts), Endposition (links) 


\section{Crunch}

Der Crunch trainiert die gesamte hintere Kette von der Schulter über den Rücken, Gesäß, Oberschenkel bis zum Unterschenkel. Außerdem ist ein sehr hoher koordinativer Anspruch durch eine hohe Gleichgewichtsleistung und die diagonale Arbeitsweise gegeben.

Die innervierte Muskelschlinge arbeitet vom oberen Rücken und Schulterbereich, M. deltoideus (Pars acromialis $\mathrm{m}$. deltoidei, Pars spinalis $\mathrm{m}$. deltoidei) und M. trapezius, weiter über den M. longissimus. Weiterhin werden der M. gluteus maximus und die gesamte Ischiocurale Muskulatur, hauptsächlich der M. biceps femoris zur Hüftstreckung innerviert.
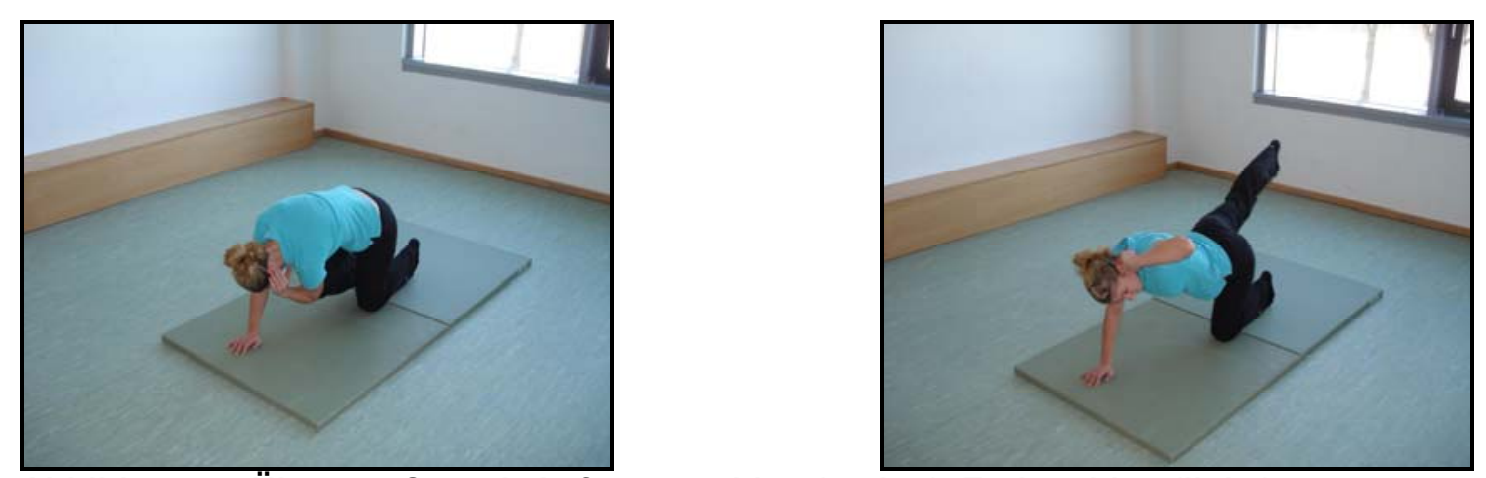

Abbildung 5: Übung 2 Crunch Anfangsposition (rechts), Endposition (links) 


\section{Sit Up (gerade)}

Die Sit ups trainieren nahezu isoliert den geraden Bauchmuskel (M. rectus abdominis). Unterstützend innerviert werden der äußere und der innere schräge Bauchmuskel (M. obliquus externus abdominis, M obliquus internus abdominis).

Als rumpfstabilisierende Muskulatur ist die Bauchmuskulatur besonders wichtig. Mit der Position der Arme kann die Schwierigkeit variiert werden. Eine Erhöhung der Schwierigkeit wird durch die Entfernung der Armhaltung vom Körperschwerpunkt erzeugt (bspw. vor der Brust gekreuzte Arme).
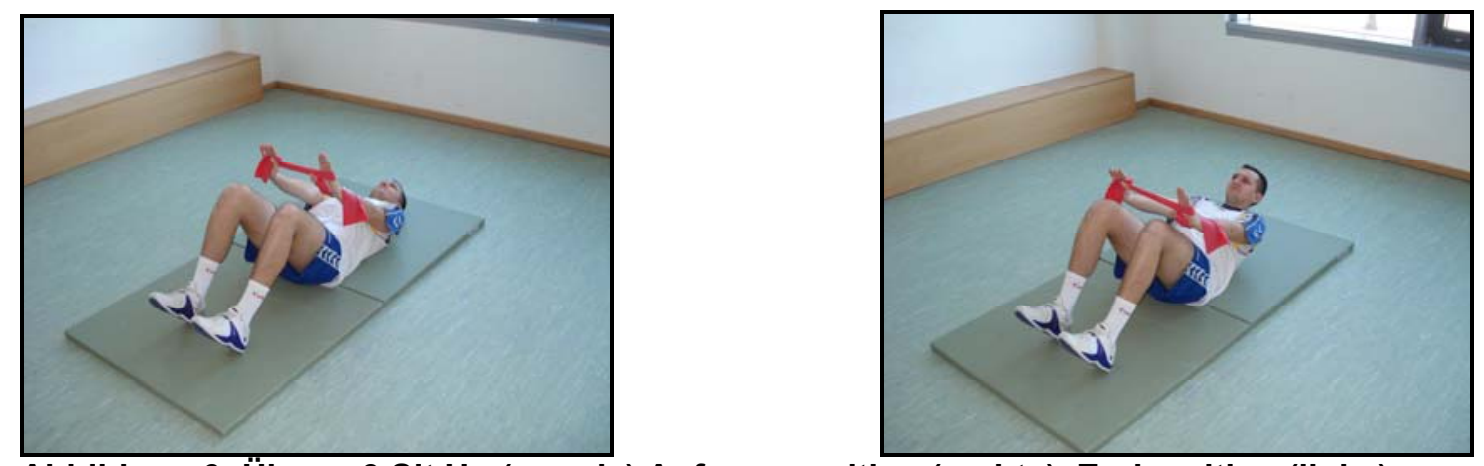

Abbildung 6: Übung 3 Sit Up (gerade) Anfangsposition (rechts), Endposition (links)

\section{Sit Up (schräg)}

Der schräge Sit Up innerviert maximal den M. obliquus externus abdominis und den M obliquus internus abdominis. Zusätzlich wird der $\mathrm{M}$. rectus abdominis trainiert.

Beide Sit Up Übungen kräftigen isoliert die Bauchmuskulatur, da diese oftmals zu schwach ist. Daraus resultieren nicht selten Schmerzen im Bereich des unteren Rückens. Die Variation der Schwierigkeit ist identisch zur vorherigen Übung.
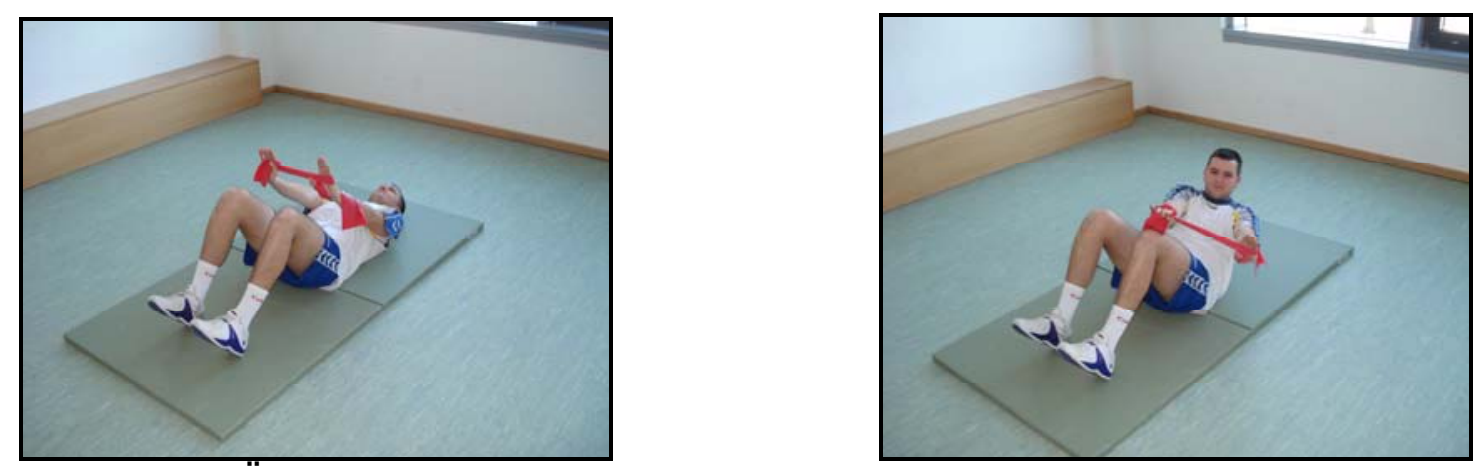

Abbildung 7: Übung 4 Sit Up (schräg) Anfangsposition (rechts), Endposition (links) 


\section{Liegestütz}

Der Liegestütz kräftigt die Arm- und Brustmuskulatur. Hauptsächlich arbeiten der M. pectoralis major, der M. deltoideus (Pars claviculare) und der M. triceps brachii. Eine Erhöhung der Schwierigkeit kann durch einen größeren Abstand zwischen Knien und Händen erreicht werden. Dadurch kommt es zu einer anderen Gewichtsverteilung und der Schweregrad der Übung erhöht sich.
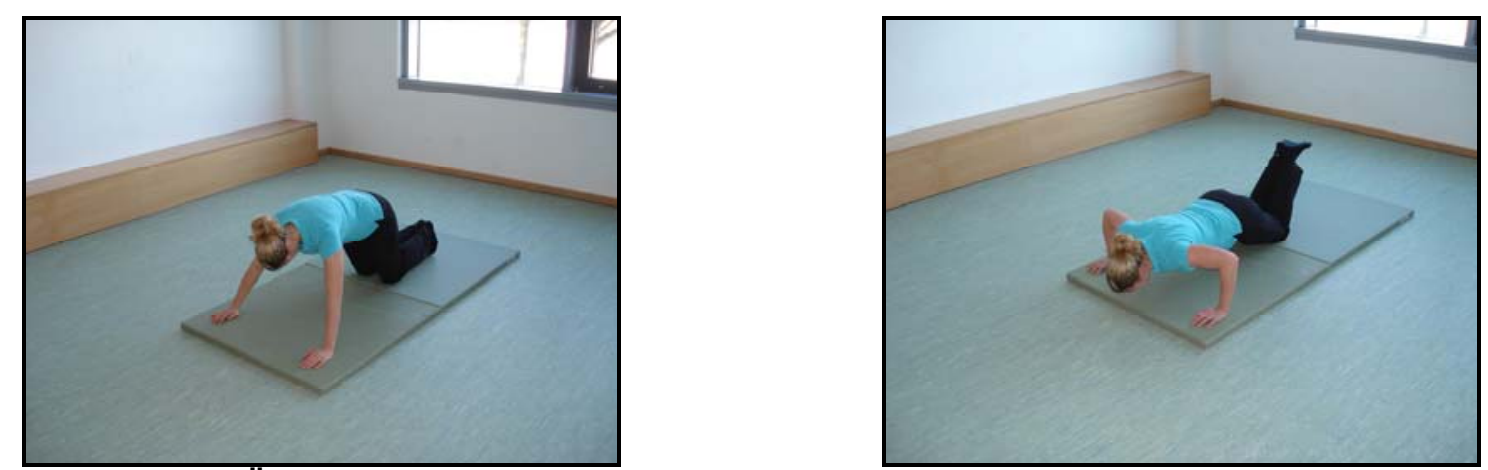

Abbildung 8: Übung 5 Liegestütz Anfangsposition (rechts), Endposition (links)

\section{Latzug}

Die Übung trainiert vornehmlich den M. latissimus dorsi und den M. biceps brachii. In der Ausgangsstellung sind aber durch die Überkopfhaltung der Arme auch der M. trapezius und der M. deltoideus (Pars claviculare deltoidei) innerviert. Eine Regulation der Schwierigkeit erfolgt über die Länge des Therra-Bandes.
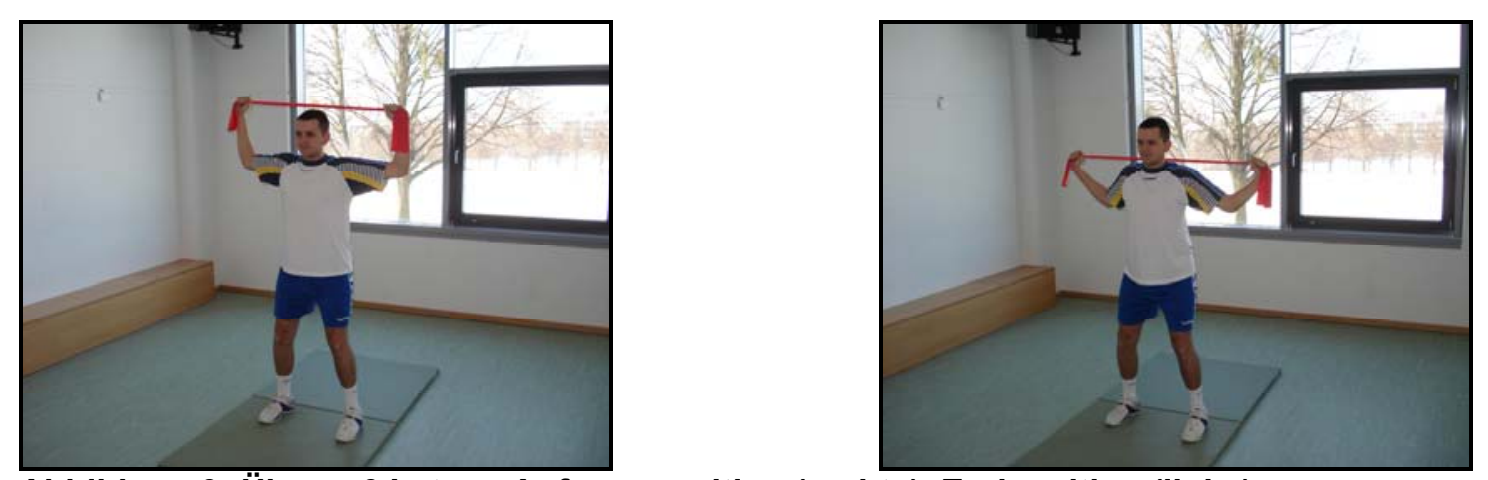

Abbildung 9: Übung 6 Latzug Anfangsposition (rechts), Endposition (links) 


\section{Seitheben}

Das Seitheben mit Rumpfrotation kräftigt in erster Linie die Schultermuskulatur. Hauptsächlich innerviert sind der M. deltoideus in allen drei Anteilen sowie Teile der Rotatorenmanschette (M. supraspinatus und Infraspinatus). Außerdem wird durch die Rumpfrotation die schräge Bauchmuskulatur (M. obliquus internus abdominis, $M$. obliquus externus externus) gekräftigt. Die Regulation der Schwierigkeit erfolgt ebenfalls über die Länge des Therra-Bandes.
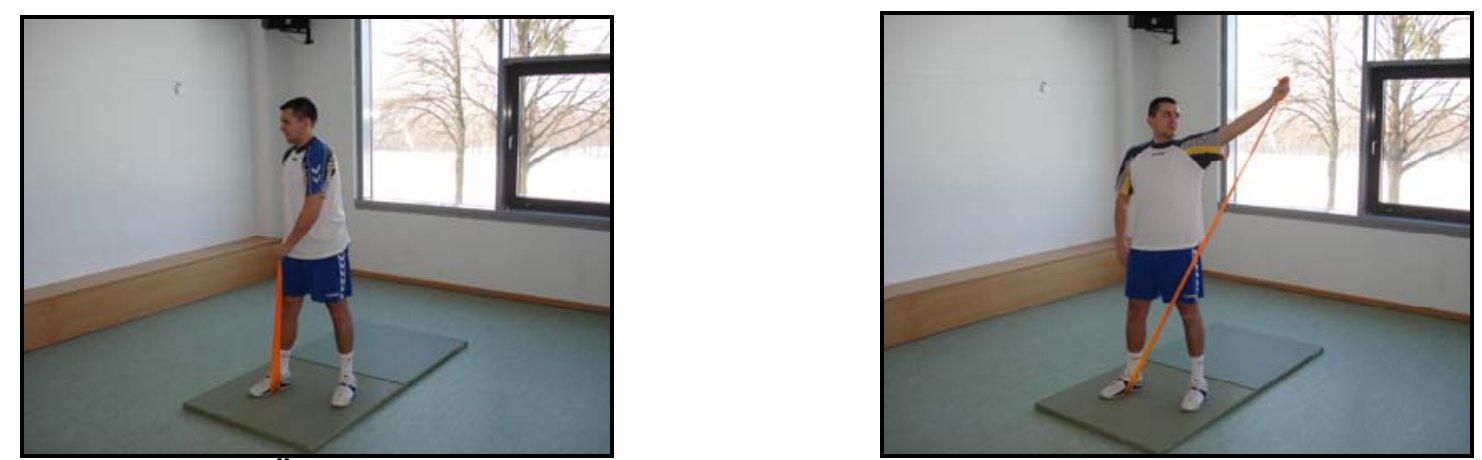

Abbildung 10: Übung 7 Seitheben Anfangsposition (rechts), Endposition (links)

\section{Ruderzug}

Der Ruderzug trainiert die gesamte obere Rückenmuskulatur sowie den M. biceps brachii. Die größte Innervation hat der M. trapezius, der M. latissimus dorsi, der M. rhomboideus major sowie der M. deltoideus (Pars spinalis m. deltoidei). Durch die Körperhaltung wird auch der lange Rückenstrecker (M. longissimus dorsi) innerviert. Der Schwierigkeitsgrad bestimmt sich erneut über die Länge des Therra-Bandes.
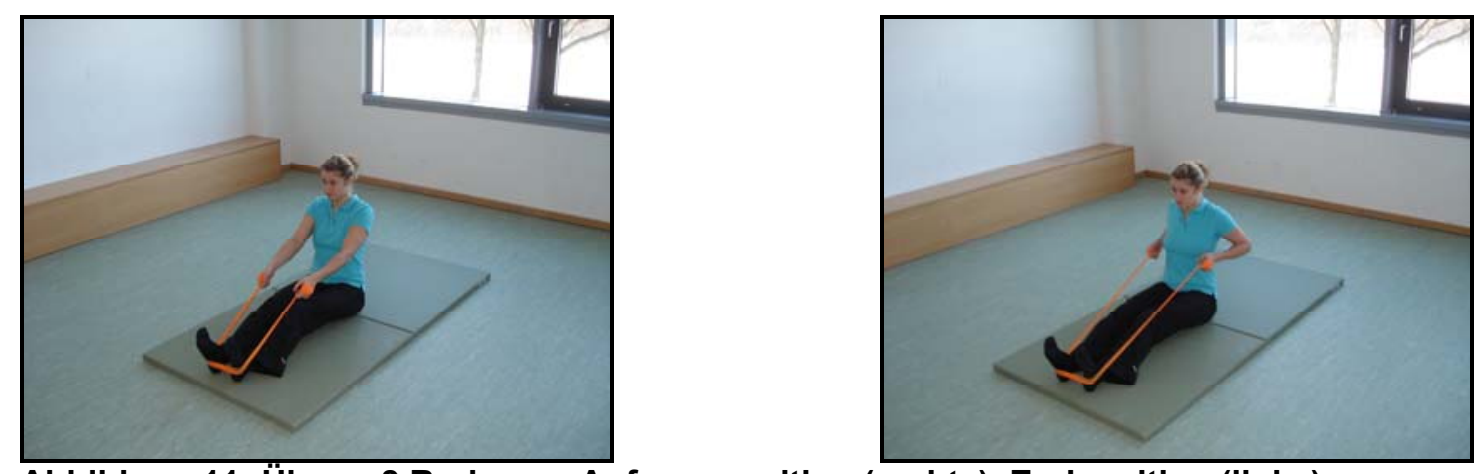

Abbildung 11: Übung 8 Ruderzug Anfangsposition (rechts), Endposition (links) 
Die vorgenommene Charakterisierung, der bei den Übungen innervierten Muskulatur ist nicht präzise. Sämtliche Übungen innervieren darüber hinaus zahlreiche kleinere Hilfsmuskeln. Diese aufzuführen wäre jedoch zu umfangreich und für das Thema der Arbeit nicht sinnvoll.

Das Übungsmanual ist im Anhang unter Anlage 11 eingestellt. In der Rehabilitation wurde zum Teil unter Aufsicht trainiert. Dadurch sollte gesichert werden, dass die Übungen auch in der Heimtrainingsphase korrekt ausgeführt werden.

\subsection{Studienziele}

Bisher findet man nur wenig Literatur darüber, wie sich Krafttraining auf Fatigueparameter und Parameter der Lebensqualität auswirkt. Die Wirkung von Ausdauertraining wurde bereits weitaus intensiver untersucht. Zudem ist bislang weitestgehend ungeklärt wie der Tagesverlauf der Fatigue von Tumorerkrankten mit akuter chronischer Fatigue aussieht.

Es wurde ein Studiendesign entwickelt, um Teilfragen dieses Themas beantworten zu können und weitere Probleme in dem Zusammenhang offen zulegen.

\section{Hauptziel der Studie}

Das Hauptziel der Studie war es, die Auswirkungen und Praktikabilität eines rehabilitativen Krafttrainings auf körperliche Leistungsfähigkeit, Fatigue und Lebensqualität innerhalb eines Zeitraums von vier Monaten zu untersuchen. Zusätzlich sollten Rückschlüsse darauf gezogen werden, wie sich ein rehabilitatives Krafttraining auf die Tagesrhythmik der Fatigue bei Patienten mit Tumorerkrankungen auswirkte.

\section{Teilziele der Studie}

- Vollerhebung der Fatigue in der Rehabilitationsklinik im Studienzeitraum

- Entwicklung eines Tagesrhythmikschemas der Fatigue

- Überprüfung subjektiver und objektiver Auswirkungen der Rehabilitation und des Trainingsprogramms auf Kraft- und Ausdauerparameter 
- Ermittlung von kurz- und mittelfristigen Veränderungen von Fatigueparametern durch das Trainingsprogramm

- Betrachtung von Motivation und Therapietreue während des gesamten Studienverlaufs

\subsection{Patientenrekrutierung und Dauer der Studie}

Die Studie wurde mit einer zufälligen ausgewählten Gruppe onkologischer Patienten in stationärer Rehabilitation durchgeführt. Mit dem Anreisetag bekamen alle Patienten einen Fatigue-LASA ausgehändigt. Patienten mit einem Score $\geq 4$ wurden zu einer Informationsveranstaltung eingeladen.

Teilnehmende Patienten wurden monatsweise blockrandomisiert und in Kontroll- und Trainingsgruppe eingeteilt. Die Zuteilung von Monat und zu testender Gruppe wurde zufällig gewählt.

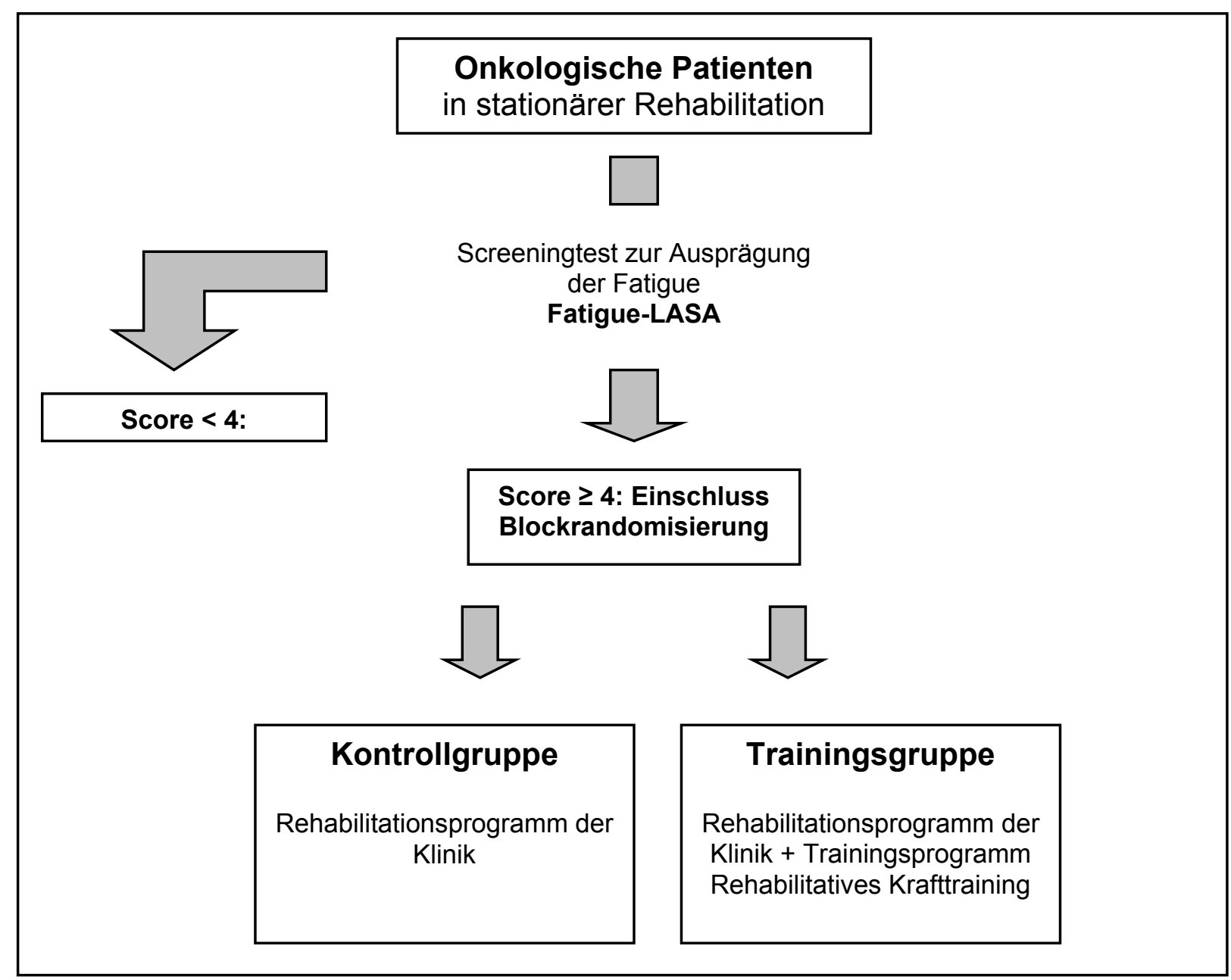

Abbildung 12: Patientenrekrutierung 
Bei der Patientenrekrutierung galten die folgenden Einschluss- bzw. Ausschlusskriterien:

5.4.1 Einschlusskriterien:

- Erkrankung an Krebs

- Aufenthalt in der stationären Rehabilitation für mindestens drei Wochen

- Score von mindestens vier auf dem Fatigue-LASA Bogen

- Alter $\leq 75$ Jahren

5.4.2 Ausschlusskriterien:

- Fehlende Einwilligungserklärung

- Alter > 75 Jahren

- Mindesttherapieaufenthalt von drei Wochen

- Bauchschnitt während der letzten sechs Monate

- Schwerwiegende Begleiterkrankungen oder Funktionsstörungen, die eine Durchführung des Trainings nicht zulassen

- Laufende Chemotherapie

Der zeitliche Umfang der Studie umfasste für die Patienten drei Wochen der stationären Rehabilitation und die darauf folgenden drei Monate. Der Untersuchungszeitraum ging von Januar 2009 bis August 2010.

Traten ein oder mehrere der folgenden Umstände auf, führte das zu einem Abbruch der Studie für den jeweiligen Probanden. Ein solcher Fall wurde als drop out gewertet:

- Rücknahme der Einwilligung

- Auftreten einer Erkrankung oder eines Rezidiv

- Verletzung des Studienprotokolls: bspw. fehlende Unterlagen

- Auftreten eines Ausschlusskriteriums

- Umstände, die sich bei Weiterführung der Studie gesundheitsgefährdend auf den Probanden auswirken könnten 


\subsection{Ablauf der Studie}

In Vorbereitung der Studie wurde zunächst ein Pre-Test mit 30 Patienten durchgeführt. Hierzu wurden alle anreisenden Patienten im Rahmen der üblichen Eingangsdiagnostik mittels einer Visualanalogskala (Fatigue-LASA) gescreent. Anschließend wurden alle Patienten, die sich stärker erschöpft fühlten (Fatigue Score $\geq 4$ ) zu einem Informationsgespräch eingeladen. Im Zuge dieses Gesprächs wurden Informationen zu der Untersuchung gegeben. Zusätzlich bekam jeder Patient ein Aufklärungsschreiben und eine Einwilligungserklärung ausgehändigt.

Teilnehmende Patienten wurden anschließend zu Beginn (T1) und zum Ende (T2) der Rehabilitation mit einem Screeningbogen zur Fatiguerhythmik befragt. Patienten, die nicht teilnehmen wollten oder Einschlusskriterien nicht erfüllten, wurden von der Untersuchung ausgeschlossen.

Nach Beendigung des Pre-Tests begann die Hauptuntersuchung. Die Vorgehensweise zur Rekrutierung der Probanden war identisch zum Pre-Test. Es wurde ebenfalls allen anreisenden Patienten ein Fatigue-LASA vorgelegt und anschließend ein Informationsgespräch durchgeführt. Patienten, die sich zur Teilnahme entschlossen und die Einschlusskriterien erfüllten, erhielten ein Aufklärungsschreiben und eine Einwilligungserklärung.

Um unerwünschte verfälschende Effekte zu vermeiden, wurden die Patienten randomisiert und in Kontroll- und Trainingsgruppe eingeteilt. Die Randomisierung erfolgte monatsweise im Block und mit einer Zufallsliste.

Die Kontrollgruppe führte das übliche Sport- und Rehabilitationsprogramm der Sonnenberg-Klinik aus, während die Trainingsgruppe darüber hinaus zusätzlich ein rehabilitatives Krafttraining absolvierte. Das Trainingsprogramm wurde während der gesamten Studiendauer zwei- bis dreimal wöchentlich je 30 Minuten ausgeführt.

Die Messmethoden zur Datenerhebung waren in beiden Gruppen absolut identisch.

Zum Messzeitpunkt T1 (Beginn der Rehabilitation) bekamen alle Probanden den Fragebogen T1 bestehend aus Dokumentationsbogen T1, Screeningbogen Fatiguerhythmik, HADS (Hospital Anxiety and Depression Scale), MFI 
(Multidimensional Fatigue Inventory), EORTC QLQ-C30, und FACIT-F (Functional Assessment of Chronic Illness Therapy Measurement System-Fatigue).

Zusätzlich wurde mit allen Probanden eine Leistungsdiagnostik durchgeführt. Es handelte sich um drei leistungsdiagnostische Testverfahren zur Messung von Ausdauer (Physical Working Capacity 130, 6-Minuten Gehtest) und Kraft (Kraftmessung mit Diggi Max Kraftmessdose).

Anschließend wurde die Kontrollgruppe in die Rehabilitation entlassen.

Die Trainingsgruppe erhielt eine Einweisung in das Trainingsprogramm und das dazu ausgehändigte Trainingsmanual. In diesem wurde die Ausführung der Übungen in Wort und Schrift erklärt. Zusätzlich wurde den Probanden ein ca. 1,60 m langes Therra-Band ausgehändigt.

Es gab drei festgelegte Zeiten pro Woche, in denen die Übungen durchgeführt werden sollten. Zum Teil wurde unter Aufsicht eines Übungsleiters trainiert, sonst in Eigenverantwortung. Die Vorgabe bestand in zwei bis drei Trainingseinheiten pro Woche.

Drei Wochen später folgte mit der Entlassung aus der Klinik der Messzeitpunkt T2. Die Probanden beider Gruppen wurden erneut zur Leistungsdiagnostik, bestehend aus den drei oben genannten Leistungstests, geladen. Die Differenzen der Messwerte beider Tests gaben Aufschluss über die Veränderung von Kraft und Ausdauer während der Rehabilitation. Zusätzlich bekamen die Probanden den Fragebogen T2, bestehend aus Dokumentationsbogen T2, Screeningbogen Fatiguerhythmik, HADS, MFI, EORTC QLQ-C30, und FACIT-F, ausgehändigt.

Patienten der Trainingsgruppe bekamen Dokumentationsbögen zum Training, in denen das Training mit Datum, Uhrzeit, Umfang und Intensität während der drei folgenden Monate genau festgehalten werden sollte.

Drei Monate nach Abreise aus der Sonnenberg-Klinik, zum Messzeitpunkt T3, wurde eine postalische Befragung aller Probanden beider Gruppen durchgeführt. Der Fragebogen T3 (Dokumentationsbogen T3, Screeningbogen Fatiguerhythmik, HADS, MFI, EORTC QLQ-C30, und FACIT-F) wurde zusammen mit einem frankierten Rückumschlag an die Probanden versendet. 
Neben dem Fragebogen T3 wurden von der Trainingsgruppe auch die Dokumentationsbögen zum Training wieder zurück geschickt. Mit Zurücksenden der Unterlagen kam es zum Abschluss der Studie.

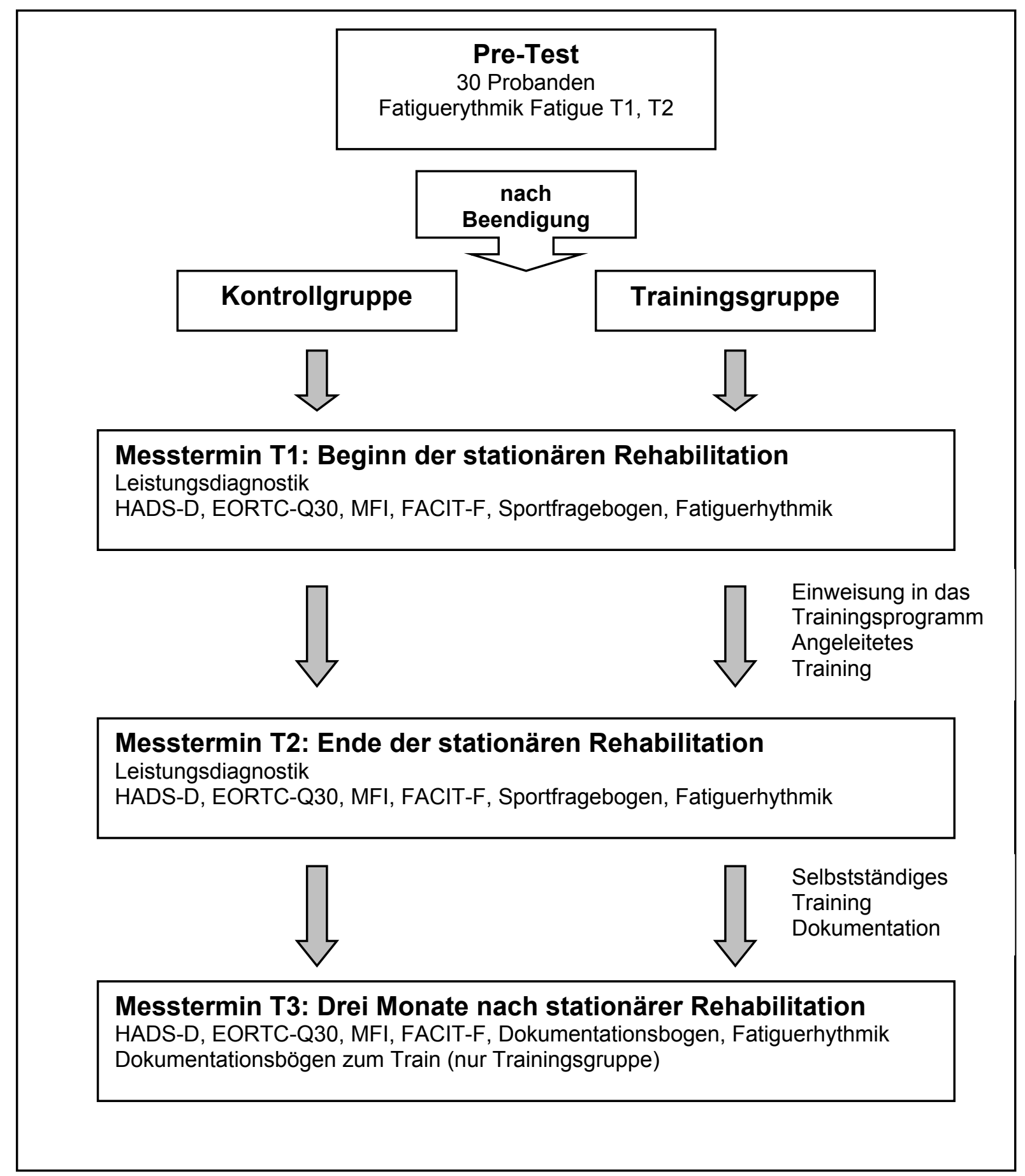

Abbildung 13: Ablauf der Studie 


\subsection{Material und Methoden}

Zur Überprüfung der Wirkung des Trainingsprogramms kamen verschiedene leistungsdiagnostische und empirische Messmethoden zum Einsatz.

Es wurden leistungsdiagnostische Testverfahren verwendet, um Veränderungen im Ausdauer- und Kraftbereich messen und objektiv bewerten zu können.

Standardisierte Fragebögen mit unterschiedlichen Schwerpunkten kamen für die Messung von Fatigue und Lebensqualität zur Anwendung.

\subsubsection{Fragebögen}

Mit Anreise in die Sonnenberg-Klinik wurden alle Patienten mit dem Fatigue-LASA einem Screening unterzogen. Anschließend bekamen teilnehmende Patienten eine Zusammenstellung bestehend aus vier standardisierten Fragebögen. Zusätzlich waren ein Screeningbogen zur Fatiguerhythmik für drei Tage und ein selbstentwickelter Dokumentationsbogen enthalten.

Befragt wurden die Patienten zu drei Messzeitpunkten, zu Beginn der Rehabilitation (T1), zum Ende der Rehabilitation (T2) und drei Monate nach T2 (T3).

\subsubsection{Fatigue-LASA (Linear-Analog-Self-Assessment-Scale)}

Der Fatigue-LASA ist eine lineare, analoge Selbstbeurteilungsskala. Die Angaben sind subjektiv und werden anhand von Distanzmaßnahmen auf einer Linie abgetragen. An den Endpunkten der Linie stehen jeweils gegensätzliche Aussagen, die dazwischen mit Linienpunkten gegliedert werden (Abb. 14).

Die Fatigue-LASA Skala hat den Vorteil, dass sie leicht verständlich und sehr schnell zu bearbeiten ist.

Eine valide und qualitativ zuverlässige Messung der Fatigue wird durch den Messbogen garantiert.

Der Fatigue-LASA wurde als Screeninginstrument während der gesamten Untersuchungszeit bei Anreise an alle Patienten der Sonnenberg-Klinik verteilt und als Vollerhebung ausgewertet. 
Außerdem wurden Patienten mit einer Score $\geq 4 \mathrm{zu}$ einem Informationsgespräch eingeladen, um Probanden für die Untersuchung zu rekrutieren. Der Fatigue-LASA ist unter Anlage $3 \mathrm{im}$ Anhang zu finden.

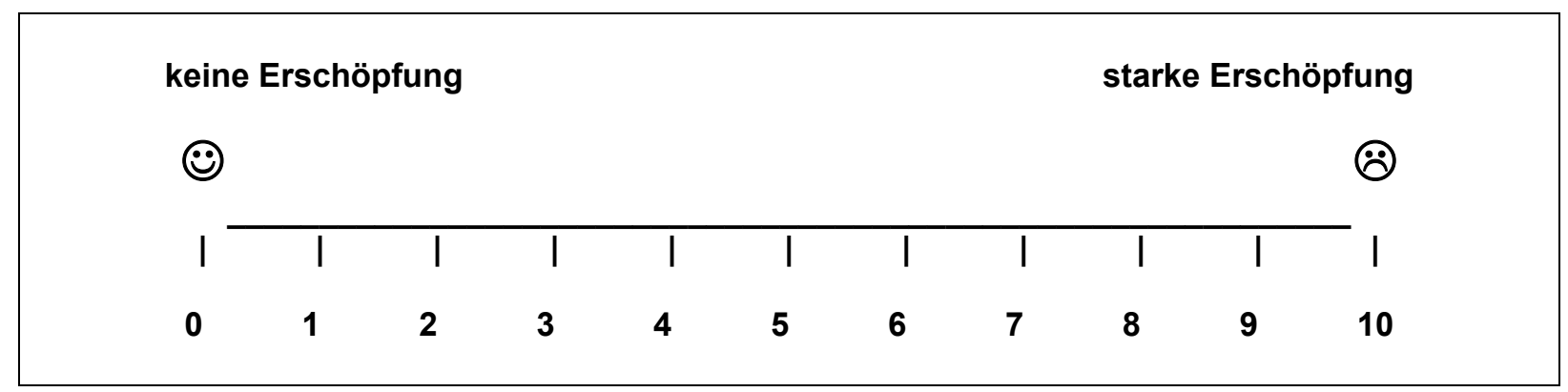

Abbildung 14: Fatigue-LASA Skala

\subsubsection{Screeningbogen zur Fatiguerhythmik}

Mit dem Screeningbogen zur Fatiguerhythmik sollte der Energieverlauf von Patienten mit tumorbedingter Fatigue untersucht werden. Prinzipiell handelte es sich dabei um eine LASA-Skala, die zu fünf verschiedenen Tageszeitpunkten erhoben wurde (8.00 Uhr, 11.00 Uhr, 14.00 Uhr, 17.00 Uhr, 21.00 Uhr). Dadurch sollten sich Rückschlüsse über den Fatigueverlauf während des Tages ziehen lassen. Im Umkehrschluss sollten Energieverlaufserkenntnisse ableitbar sein.

Zusätzlich wurden noch LASA Skalen zur Schlafqualität und zum Aktivitätslevel abgefragt.

Die Schlafqualität sollte subjektiv unter Einbeziehung der Kriterien Einschlafstörungen, Durchschlafstörungen und Erholungsqualität von Schlaf bewertet werden.

Der Aktivitätslevel ist eine subjektive Skala zur Messung von Motivation und Interesse an der Ausführung verschiedenster Aktivitäten. Sowohl die LASA-Skala zur Schlafqualität als auch die zum Aktivitätslevel müssen in der Auswertung umcodiert werden. Hohe Scores werden positiv bewertet.

Der Screeningbogen zur Fatiguerhythmik kam schon im Pre-Test zur Anwendung. Hier wurde er zu T1 und T2 erhoben. 
Um die Genauigkeit der Angaben möglichst hoch zu halten, wurde zu jedem Messzeitpunkt an drei aufeinander folgenden Tagen gemessen und die Werte anschließend gemittelt.

Während der Studie wurde der Screeningbogen zur Fatiguerhythmik an allen drei Messzeitpunkten erhoben. Die Erhebungsmodalitäten waren dabei identisch denen im Pre-Test. Der Screeningbogen zur Fatiguerhythmik ist im Anhang unter Anlage 4 zu finden.

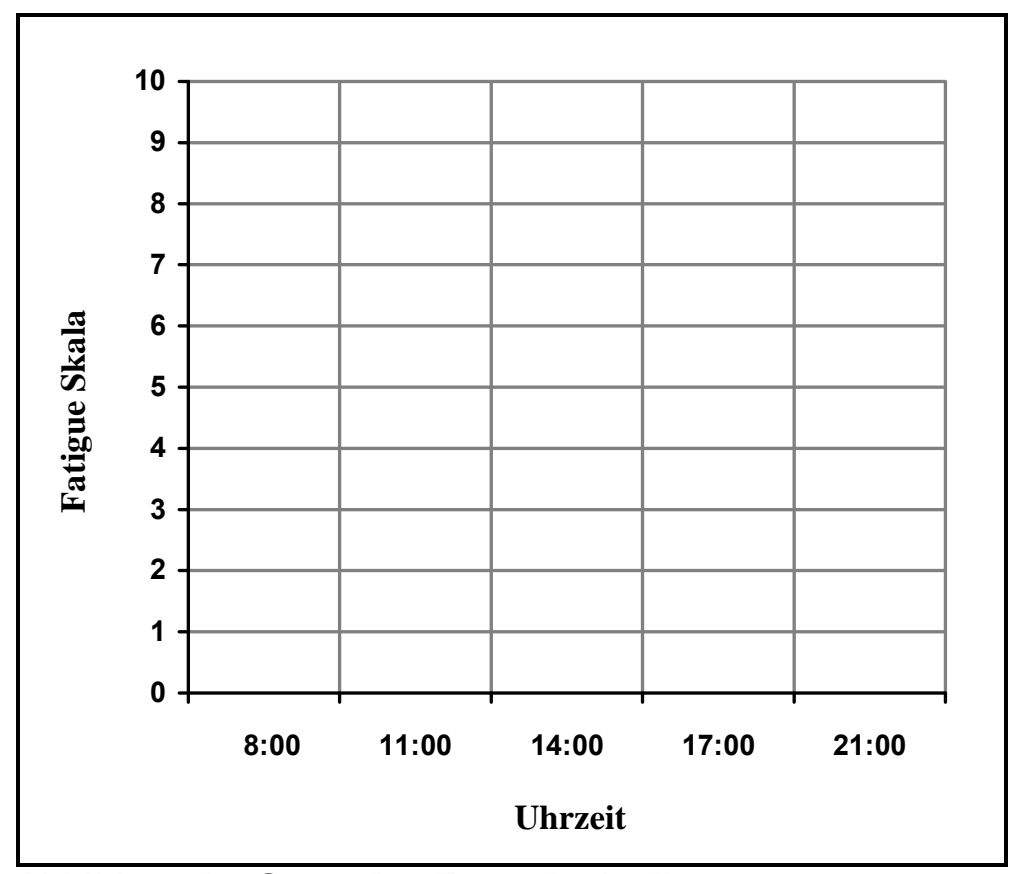

Abbildung 15: Screening Tagesrhythmik

\subsubsection{Dokumentationsbogen}

Im Verlauf der Studie gab es zu jedem Messzeitpunkt einen individuellen Dokumentationsbogen, in dem zusätzliche Fragen zu Erkrankung, Motivation und sportlicher Aktivität enthalten waren.

Zusätzlich wurden zu T1 soziodemografische Daten wie Alter, Größe, Gewicht, berufliche Situation usw. abgefragt.

Einige Fragen wiederholten sich zu allen Messzeitpunkten um Vergleiche zu ermöglichen. Informationen zur Erkrankung, Therapierung und Medikamentation 
wurden der Krankenakte entnommen und festgehalten. Die Dokumentationsbögen sind im Anhang unter den Anlagen 5, 6 und 7 zu finden.

\subsubsection{HADS-D (Hospital Anxiety and Depression Scale)}

Der HADS-D ist die gleichwertige deutsche Version des von Zigmond und Snaith 1983 entwickelten englischen HADS und wurde 1991 von Hermann vorgestellt (HERRMANN et al. 1991). Der HADS ist ein Fragebogeninstrument zur Selbstbeurteilung von Angst und Depression bei Erwachsenen mit körperlichen Beschwerden oder Erkrankungen. Seine Skala besteht aus 14 Items, von denen jeweils sieben eine Angst- und eine Depressionssubskala bilden. Die Antworten aus den itembezogenen, vierstufigen Antwortoptionen werden innerhalb der jeweiligen Subskala addiert und kategorisiert. Die Cutoff-Points liegen für beide Subskalen bei $\geq$ 8 für Verdachtsfälle und $\geq 11$ für sichere Fälle (BJELLAND et al. 2002). Während der Erstbehandlung werden die Cut off points leicht nach oben verschoben. Sie liegen dann bei $\geq 13$ und $\geq 16$ (SINGER et al. 2009). Der HADS kann sowohl als Screeninginstrument als auch zur Verlaufsbeurteilung eingesetzt werden und hat sich innerhalb Europas mittlerweile zum Standardverfahren zur Ermittlung von Angst und Depression entwickelt. Der HADS wurde auf die Hauptgütekriterien Objektivität, Reliabilität und Validität hin überprüft (HERMANN et al. 1997).

\subsubsection{MFI (Multidimensional Fatigue Inventory)}

Der MFI ist ein multidimensionales Fragebogeninstrument zur Selbstbeurteilung. Er besteht aus 20 Items und misst die Fatigue. Jedes Item besteht aus einer fünfstufigen Antwortskala.

Die Multidimensionalität ist durch die Dimensionen General Fatigue, Physical Fatigue, Mental Fatigue, Reduced Activity und Reduced Motivation definiert. Die Subskalen werden getrennt voneinander ausgezählt. Mittels einer Formel wird anschließend ein Fatigue Score berechnet, der sich für Screening- und Verlaufsanalysen eignet. Die Normwerte der Allgemeinbevölkerung sind unter 
anderem abhängig von Alter und Geschlecht. Beim Vergleich von Krebspatienten mit der Allgemeinbevölkerung müssen diese beiden Parameter ebenfalls berücksichtigt werden (SCHWARZ et al. 2003).

Der MFI unterliegt den Hauptgütekriterien (Objektivität, Reliabilität, Validität) (SMETS et al. 1995)

\subsubsection{EORTC QLQ-C3O}

Der EORTC QLQ-C30 ist ein Lebensqualitätsfragebogen für Krebspatienten. Er wurde von der European Organisation for Research and Treatment of Cancer über mehrere Jahre entwickelt. Die aktuellste Version 3.0 kommt seit 1997 zur Anwendung.

Bei dem Konzept des Fragebogens wird von einem "integrated modular approach“ gesprochen, d. h. das Kernmodul (C30) kann durch verschiedene tumorspezifische Module ergänzt werden.

Das Ergebnis stellt sich aus mehreren Multiskalen zusammen. Hierbei wird in eine Globale Gesundheitsskala, fünf Funktionsskalen (z. B. Physical Functioning, Emotional Functioning) und neun Symptomskalen (z. B. Fatigue, Pain, Insomnia) unterschieden.

Anschließend werden die Scores aus den Subskalen mit Hilfe einer Auswertungsformel berechnet. Der EORTC wurde in zahlreichen Studien verwendet und ist ein Werkzeug zur Messung von Fatigue und Lebensqualität (MINTON \& STONE 2009).

Die Hauptgütekriterien wurden in diversen Studien überprüft (AARONSON et al. 1993). 


\subsubsection{FACIT-F (Functional Assessment of Chronic Illness Therapy - Fatigue)}

Der FACIT ist ein standardisiertes Fragebogeninstrument, um gesundheitsbezogene Lebensqualität bei chronisch Kranken abzufragen. Die Entwicklung geht zurück auf David Cella, der den Fragebogen Ende der 80er Jahre entwickelte, um Lebensqualitätsparameter bei Krebspatienten abzufragen (Functional Assessment of Cancer Therapy). Bis 1997 wurde das Instrument weiterentwickelt und kommt bei unterschiedlichsten chronischen Krankheiten wie Krebs, HIV, Multiple Sklerose oder Parkinson zum Einsatz (WEBSTER et al. 2003; CELLA 1997).

Im Besonderen ist anzumerken, dass es sich um ein multidimensionales Fragebogensystem handelt, um dem multidimensionalen Kontext, in dem Lebensqualität steht, gerecht zu werden. Die vier zu Grunde liegenden Subskalen sind Körperliches Wohlbefinden, Verhältnis zu Freunden, Bekannten und ihrer Familie, Seelisches Wohlbefinden und Funktionsfähigkeit. Diese werden je nach Krankheit bzw. Krebsart durch einzelne Zusatzmodule ergänzt. In diesem Fall handelt es sich bei dem FACIT-F um das Zusatzmodul für Patienten, die unter tumorbedingter Fatigue leiden. Dabei werden körperliche Aspekte der Fatigue in Verbindung $\mathrm{zu}$ ihren funktionellen und sozialen Auswirkungen gemessen. Der Fatiguezusatz besteht aus 13 Items und ist für verschiedenste Krebspatienten in Therapie validiert (MINTON \& STONE 2009).

\subsubsection{Trainingsprotokoll}

Die Patienten der Trainingsgruppe bekamen zusammen mit dem Übungsmanual Dokumentationsbögen zum Training für drei Monate ausgehändigt. Während der Rehabilitation fand die Eingewöhnungsphase statt. Das Training wurde in dieser Zeit nicht protokolliert.

Der Dokumentationsbogen bestand aus zwei Teilen. Auf dem ersten Teil des Dokumentationsbogens wurden neben Datum und Uhrzeit des Trainings zu jeder der acht Übungen Umfang und die Intensität dokumentiert. Teil zwei des Dokumentationsbogens protokollierte sonstige sportliche Aktivitäten mit Datum und Dauer. 
Mit Hilfe der detaillierten Dokumentation des Trainings konnten Rückschlüsse auf Trainingstreue und eventuelle Leistungsveränderungen gezogen werden. Außerdem wurde ersichtlich, welche Übungen einen hohen Schwierigkeitsgrad für die Patienten hatten. Die Dokumentationsbögen sind unter Anlage $10 \mathrm{im}$ Anhang zu finden.

\subsubsection{Leistungsdiagnostik}

Eine Leistungsdiagnostik verfolgt in der Trainingspraxis im Wesentlichen zwei Ziele. Eines besteht in der Herstellung eines Ist-Soll-Vergleichs und das andere in der Kontrolle des Trainingserfolges (HOHMANN et al. 2002).

Aufschluss über Parameter der Leistungsdiagnostik kann durch Fragebögen sowie standardisierten Testverfahren erfolgen (SCHNABEL et al. 2008). Zu beachten ist allerdings, dass Fragebögen durch den Einfluss persönlichen Empfindens zumeist nur subjektive Ergebnisse liefern. Dem gegenüber ist bei standardisierten Tests mit objektiven oder zumindest semiobjektiven Werten über Ausdauer- und Kraftleistungsfähigkeit zu rechnen.

Im Allgemeinen kann davon ausgegangen werden, dass der Leistungszuwachs höher ausfällt, je niedriger die Ausgangswerte sind.

\subsubsection{Physical Working Capacity (PWC) Test}

Beim PWC Test handelt es sich um einen Fahrradergometertest im Sitzen, der die submaximale ausdauerbezogene Ausbelastung der Leistungsbeurteilung zu Grunde legt. Ergometertestvarianten haben sich in Bereichen der Fitness, Gesundheit und Arbeitsmedizin aufgrund ihrer Effizienz und hohen Praktikabilität durchgesetzt. Allerdings konnte schon vor einiger Zeit in Studien gezeigt werden, dass einzeln durchgeführte PWC-Tests keine verlässlichen Aussagen über den Leistungsstand von Sportlern zulassen (DRESCHER \& SIMON, 1989; SCHWABERGER et al. 1983). Unter Berücksichtigung des Alters, welches bei Ausdauertestverfahren eine erhebliche Rolle spielt, ist der PWC-Test für Verlaufskontrollen jedoch ein durchaus 
geeignetes Verfahren. Aussagen über metabolische Prozesse sind dennoch nicht möglich.

Zur Durchführung der Ergometertests hat es verschiedene Vorschläge zu Belastungsstufe, Gesamtbelastungszeit und Gesamtleistung gegeben (STEMPER 1988).

Für Belastungsuntersuchungen wurde von der World Health Organisation (WHO) ein Schema vorgeschlagen, in dem der Startwert bei 25 Watt liegt und alle zwei Minuten um 25 Watt gesteigert wird. In der Durchführung wurde sich an dieses Schema angelehnt, mit dem Unterschied, dass eine Belastungsstufe von drei Minuten gewählt wurde. Am Anfang jeder Belastungsstufe wurde der Puls mittels eines Pulsfrequenzmessers auf EKG-Basis gemessen und dokumentiert. Die Dokumentationsvorlage des Testprotokolls findet sich im Anhang unter Anlage 9. Vor der Belastung wurde der Ausgangspuls und zwei Minuten nachher bei einer Belastung von 25 Watt der Erholungspuls gemessen und dokumentiert.

\subsubsection{6-Minuten Gehtest}

Der 6-Minuten Gehtest ist ein valides und reliables Instrument zur Trainingssteuerung und -kontrolle. Bei Patienten in kardiologischer Rehabilitation hat sich der 6-Minuten Gehtest schon seit einiger Zeit aufgrund des Zusammenhangs zwischen Gehstrecke und ergometrisch ermittelter Leistungsfähigkeit zur Bestimmung der funktionellen Leistungsfähigkeit bewährt (REMME \& SWEDBERG 2001). In der kardiologischen Literatur lassen sich diverse Studien finden, die einen Zusammenhang zwischen $\mathrm{VO}_{2 \max }$ und 6-Minuten-Gehtest belegen. Eine Korrelation zwischen Gehstrecke und ergometerermitteltem $\mathrm{VO}_{2 m a x}$ bei Patienten mit chronischer Herzinsuffizienz wurde in zahlreichen Studien belegt (OPASICH et al. 2001; ZUGCK et al. 2000; LUCAS et al. 1999; FAGGIANO et al. 1997; CAHALIN et al. 1996; RILEY et al. 1992; LIPKIN et al. 1986). Andere Studien zeigen eine Korrelation von 6Minuten Gehtest und laufbandermitteltem $\mathrm{VO}_{2 \max }$ bei Patienten mit koronaren Herzerkrankungen (HAMILTON \& HAENNEL 2000; RILEY et al. 1992).

Unter Berücksichtigung verschiedener Faktoren wie bspw. Alter, Gewicht und Größe lassen sich Regressionsgleichungen finden, mit denen der $\mathrm{VO}_{2 \max }$ näherungsweise 
aus den Ergebnissen des 6-Minuten Gehtest berechnet werden kann (CAHALIN et al. 1996).

Trotzdem kann der 6-Minuten Gehtest nur eine Annährung und kein Ersatz für den $\mathrm{VO}_{2 m a x}$ sein. Es kann allerdings davon ausgegangen werden, dass unter gewissen Voraussetzungen eine zuverlässige Aussage aus den Ergebnissen des 6-Minuten Gehtestes möglich erscheint. Entscheidender Vorteil des 6-Minuten Gehtests ist die Praktikabilität, da dieser schon mit geringer Anzahl an Hilfsmitteln durchführbar ist.

Das Testprotokoll zum 6-Minuten Gehtest ist im Anhang unter Anlage 8 zu finden.

\subsubsection{Krafttest}

Die Kraft wurde in der Studie mit einem Messgerät der Firma Mecha Tronic gemessen. Als Kraftsensor fungiert ein DIGIMAX Messgerät. Dabei handelt es sich um ein digitales Kraftmessgerät, das die isometrische Maximalkraft über eine Kraftmessdose misst. Die Messdose ist über USB mit einem Computersystem verbunden, das die Daten sofort mit einer entsprechenden Software verarbeitet und speichert.

Im Hinblick auf das weitere Verständnis soll zunächst die zu messende Größe definiert werden.

Isometrische Maximalkraft ist die bei einer willkürlichen maximalen statischen Muskelanspannung aufwendbare Kraft. Sie gibt den Ist-Zustand der Muskelkraft unabhängig vom Trainingszustand wieder (HOLLMANN/HETTINGER 2000, 161).

Die Messung der isometrischen Muskelkraft bietet unter standardisierten Bedingungen bei Wiederholung einen hohen Grad an Übereinstimmung und entspricht den Hauptgütekriterien (Validität, Reliabilität, Objektivität) (PFEIFER et al. 2003)

Im Folgenden werden die Messparameter für die Kraftmessungen erläutert. In der Messung der isometrischen Maximalkraft wurde sich auf zwei Muskelgruppen beschränkt, die in bestimmten Messpositionen isoliert angesprochen wurden. 
Die zu messenden Muskelgruppen waren zum einen die Armbeuger und zum anderen die Kniestrecker. In den folgenden Abbildungen sind beide Messpositionen skizziert.

Ziel der Messposition Armbeugung ist die isolierte, isometrische Kraftmessung der Armbeuger. Bei der Flexionsbewegung im Ellenbogen sind im Wesentlichen drei Muskeln beteiligt. Diese werden bei der Kraftdiagnostik als funktionelle Einheit betrachtet, sollen aber dennoch kurz beschrieben werden.

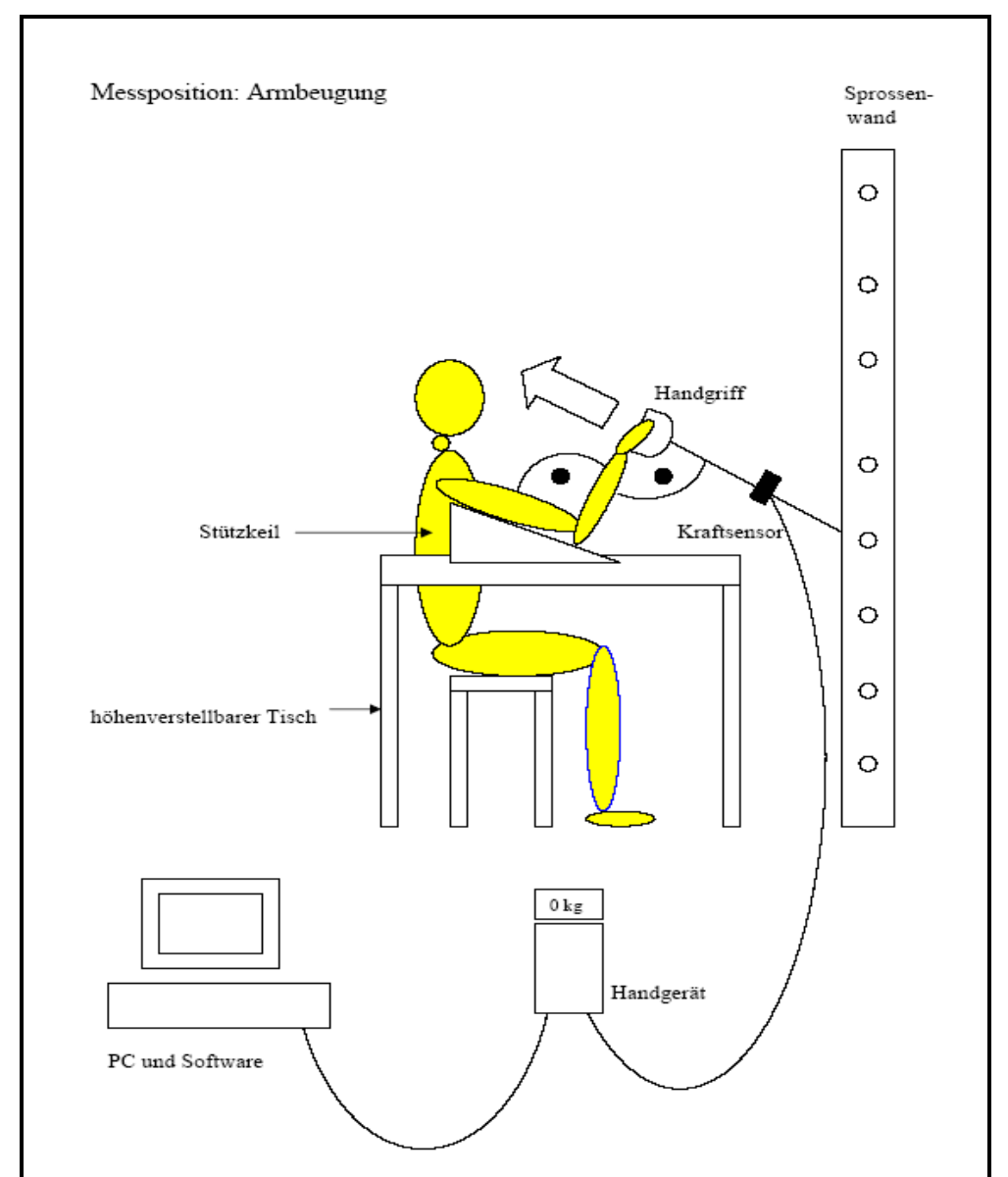

Abbildung 16: Messposition Armbeugung (ELSNER V. D. MALSBURG 2006, 83)

\section{Muskel:}

Musculus Biceps brachii

Musculus brachialis

Musculus brachioradialis

(Unterarm)

\section{Funktion:}

Flexion, Anteversion, Supination (Unterarm)

Flexion

Flexion, Supination, Pronation 
Die Messposition Kniestrecker ist die isolierte, isometrische Kraftmessung der Kniestreckmuskulatur. Für die Extension des Kniegelenks ist hauptsächlich der Musculus quadrizeps femoris verantwortlich. Dieser setzt sich aus vier Teilen zusammen, die als funktionelle Einheit die Extension des Kniegelenks ermöglichen.

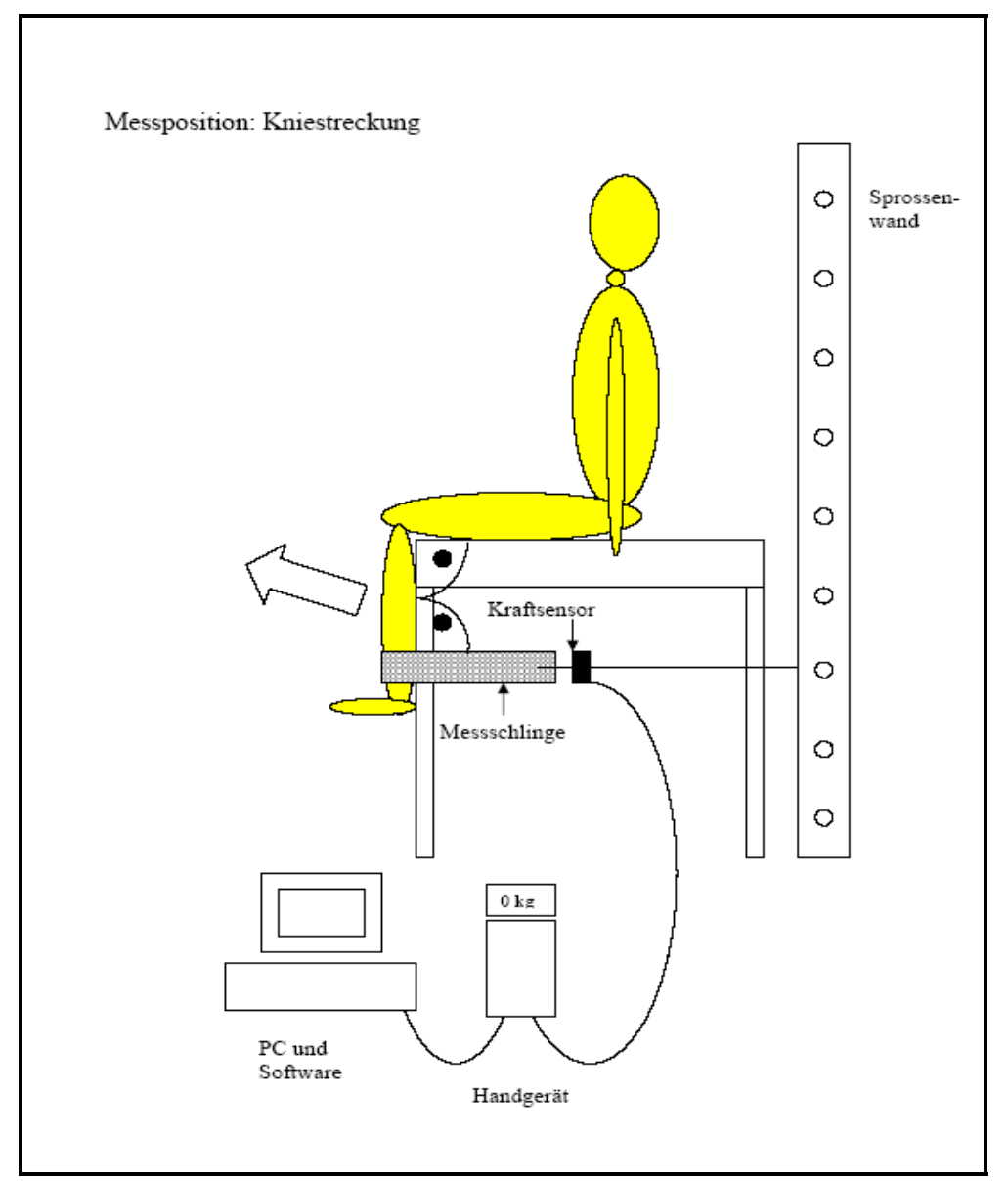

Abbildung 17: Messposition Kniestreckung (ELSNER v. d. MALSBURG 2006, 82)

\section{Muskel:}

Musculus rectus femoris

Musculus vastus medialis

Musculus vastus intermedialis

Musculus vastus lateralis

\section{Funktion:}

Extension, $\quad$ Flexion (Hüfte),

Kniegelenksstabilisierung

Extension, Kniegelenksstabilisierung

Extension, Kniegelenksstabilisierung

Extension, Kniegelenksstabilisierung 
Die Kraftdiagnostik wurde nach dem Aufbau in den Abbildungen 16 und 17 durchgeführt. Der Aufbau der Messtechnik erfolgte nach einem standardisierten Schema, um den Hauptgütekriterien zu genügen.

Im Folgenden wird der Ablauf der Messung beschrieben.

Die Personendaten wurden in die Messsoftware eingetragen. Fehlende Daten wurden zu einem späteren Zeitpunkt ergänzt.

Der Versuchsaufbau sowie die Messtechnik wurde dem Probanden erklärt und die Messposition wurde gezeigt. Die Hauptknotenpunkte der Bewegung wurden besonders akzentuiert vorgeführt.

Der Proband nahm die jeweilige Messposition ein. Fixierungsgurte und Höhe der Untersuchungsbank wurden auf den Probanden eingestellt. Ein Probeversuch wurde durchgeführt. Der Proband machte sich mit dem Test vertraut. Auf Kommando erfolgte der fünf Sekunden andauernde Test. Die Probanden wurden angehalten die Spannung nicht zu ruckartig aufzubauen. Der Kraftverlauf der Messung konnte parallel auf dem Computermonitor verfolgt werden.

Der Krafttest wurde pro Körperseite zweimal durchgeführt. Nach erfolgreicher Messung wurde der Messplatz umgebaut, um die Messung in der jeweils anderen Messposition durchzuführen.

\subsection{Statistische Auswertung der Ergebnisse}

Die statistische Auswertung der Ergebnisse erfolgte mit Hilfe der Statistiksoftware GraphPadPrism 4.0 und SPSS 11.5 for Windows.

Bei den Kraftmessungen kam zusätzlich die Digimax Software zum Einsatz.

Bei den Fragebögen HADS, MFI, EORTC QLQ-C30 und dem FACIT-F wurden die jeweiligen Scoring Guidelines der Autoren genutzt. Zum Teil mussten die erhobenen Daten zuvor umcodiert werden.

Als Auswertungsverfahren wurden Methoden der deskriptiven Statistik angewendet. Zum Vergleich der Daten untereinander wurden Signifikanztests (t-Test) 
durchgeführt. Als Signifikanzgrenze wurde eine Irrtumswahrscheinlichkeit von unter $5 \%$ festgelegt. Somit galt aus $\mathbf{p} \leq \mathbf{0 , 0 5}$ folgte Signifikanz. Als hochsignifikant wurden Ergebnisse betrachtet, deren Irrtumswahrscheinlichkeit unter 0,01\% lag, also p < 0,0001 .

\subsection{Störvariablen}

Innerhalb der Probandengruppe gab es erhebliche Altersunterschiede. Da Jüngere wesentlich kürzere Erholungszeiten nach Ausdauerbelastungen haben, könnten störende Effekte auf die Fatiguewerte aufgetreten sein.

Art und Umfang der sportlichen Aktivität vor und während der Erkrankung haben unterschiedliche Leistungsvoraussetzungen der Probanden zur Folge. Die Ausgangsvoraussetzungen waren somit sehr heterogen. Hieraus resultierten sowohl Unterschiede in der Betrachtung der leistungsdiagnostischen Ergebnisse als auch motivationale Unterschiede.

Erinnerungs- und Wiederholungseffekte bei den Befragungen können bewirken, dass Probanden Antworten unbewusst nach sozialer Erwünschtheit geben.

In der Studienzeit geschehene extreme Ereignisse (bspw. Trennung vom Lebenspartner) können Effekte auf die Lebensqualität haben.

Da die Studie über ein Jahr ging, könnten typische jahreszeitliche Stimmungsveränderungen Effekte auf die Lebensqualität und Fatigue haben.

Problematisch ist zum Teil die genaue Erfassung der körperlichen Aktivität nach der Rehabilitation. Berufliche Belastungen konnten beispielsweise nicht mit in die Überlegung einfließen. Solche wurden in der Auswertung nicht beachtet.

Trotz Einweisung und Kontrolle der Übungen aus dem Trainingsprogramm konnte nicht davon ausgegangen werden, dass die Übungen von allen Patienten fehlerfrei 
durchgeführt wurden. Daraus ergeben sich Unterschiede in der Effektivität des Trainings.

Veränderungen der Lebensqualität lassen sich im Allgemeinen nur schwer auf eine bestimmte Maßnahme zurückführen. Zudem erschwert die Tatsache der großen Fülle unterschiedlicher, den Patienten betreffender Ausgangsparameter die Untersuchung. Es wurde davon ausgegangen, dass die Summe der Interventionen den Ausschlag zur Verbesserung gibt.

Während der Rehabilitation und auch danach sind auch die Probanden der Kontrollgruppe sportlich aktiv. Außerdem ist der Zeitraum der Rehabilitation nur sehr gering. Signifikante Unterschiede in der Leistungsdiagnostik sind in beiden Gruppen nicht zu vermuten. 


\section{Ergebnisse}

Im folgenden Kapitel werden die Ergebnisse der Studie umfassend dargestellt. Dazu gehören die Ergebnisse des Pre-Tests sowie die mit Fragebogen und Leistungsdiagnostik gewonnenen Daten. Die Charakterisierung des Patientenkollektivs erfolgte mit Hilfe einer deskriptiven Statistik.

Daten der Fragebögen und der Leistungsdiagnostik wurden mittels t-Test verglichen und auf signifikante Veränderungen hin untersucht.

\subsection{Ergebnisse Pre-Test:}

\subsubsection{Beschreibung der Pre-Test Stichprobe}

Schon während des Pre-Tests wurde eine Vollerhebung mit dem Fatigue-LASA durchgeführt. Alle neu angereisten Patienten der Klinik wurden gescreent.

Die Anzahl der Probanden für die Pre-Test Erhebung zur Tagesrhythmik wurde im Vorfeld auf $\mathrm{N}=30$ festgelegt. Die Kriterien zur Teilnahme am Pre-Test waren identisch denen zur Teilnahme an der Studie (Abschnitt 5.4).

\subsubsection{Fatigue-LASA}

Während der Pre-Test Phase wurden 235 Fatigue-LASA registriert. Der Mittelwert lag bei $4,71 \pm 2,5$. Mittelwert und Standardabweichung sind in Tabelle 1 aufgeführt.

\begin{tabular}{|l|r|}
\hline Fatigue-LASA & 235 \\
\hline Anzahl Werte & 4,71 \\
\hline Mittelwert & 2,5 \\
\hline Standardabweichung & \\
\hline
\end{tabular}




\subsubsection{Tagesrhythmik Fatigue}

Der Screeningbogen zur Fatiguerhythmik wurde während des Pre-Tests von 30 Probanden an drei aufeinander folgenden Tagen ausgefüllt. In Abbildung 18 sind die Ergebnisse grafisch dargestellt.

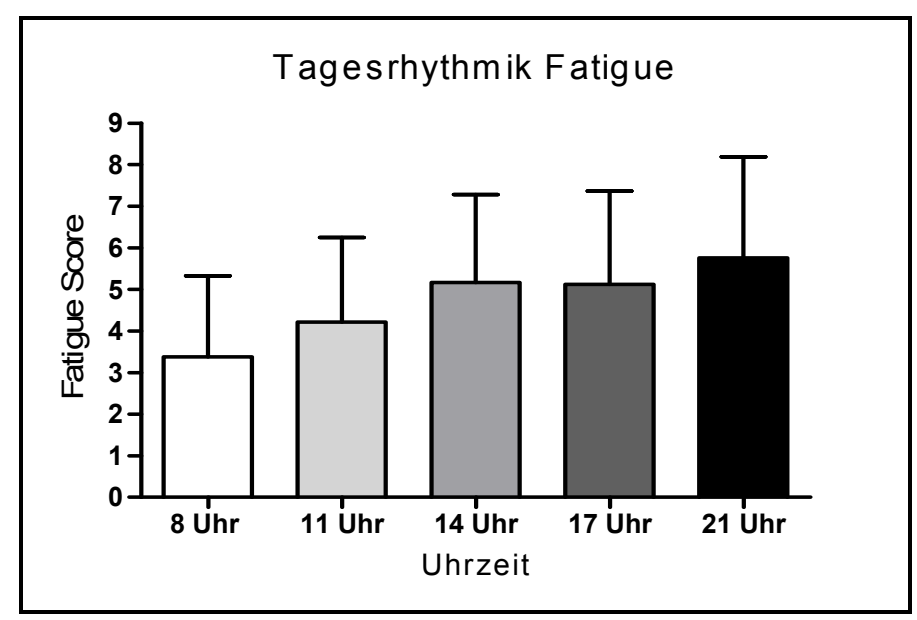

Abbildung 18: Tagesrhythmik Fatigue Pre-Test

Tabelle 2 zeigt die Mittelwerte der Fatigue mit Standardabweichung zu den einzelnen Tageszeitpunkten.

\begin{tabular}{|l|r|r|r|r|r|}
\hline Uhrzeit & \multicolumn{1}{|c|}{$\mathbf{8}$ Uhr } & 11 Uhr & \multicolumn{1}{|c|}{ 14 Uhr } & 17 Uhr & 21 Uhr \\
\hline Anzahl Werte & 90 & 90 & 90 & 90 & 90 \\
\hline Mittelwert & 3,38 & 4,21 & 5,17 & 5,12 & 5,76 \\
\hline Standardabweichung & 2,0 & 2,0 & 2,1 & 2,2 & 2,4 \\
\hline
\end{tabular}

Tabelle 2: Tagesrhythmik Fatigue Pre-Test 


\subsubsection{Schlafqualität und Aktivitätslevel}

Schlafqualität und Aktivitätslevel wurden auf dem Fatiguerhythmikbogen durch eine LASA Skala erhoben. Die Methodik war dabei identisch der Fatigueerhebung mittels Fatigue-LASA. Die Ergebnisse wurden anschließend umcodiert. Während des Pre-

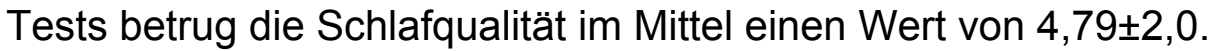

Der Mittelwert des Aktivitätslevel lag bei 5,46ะ1,9.

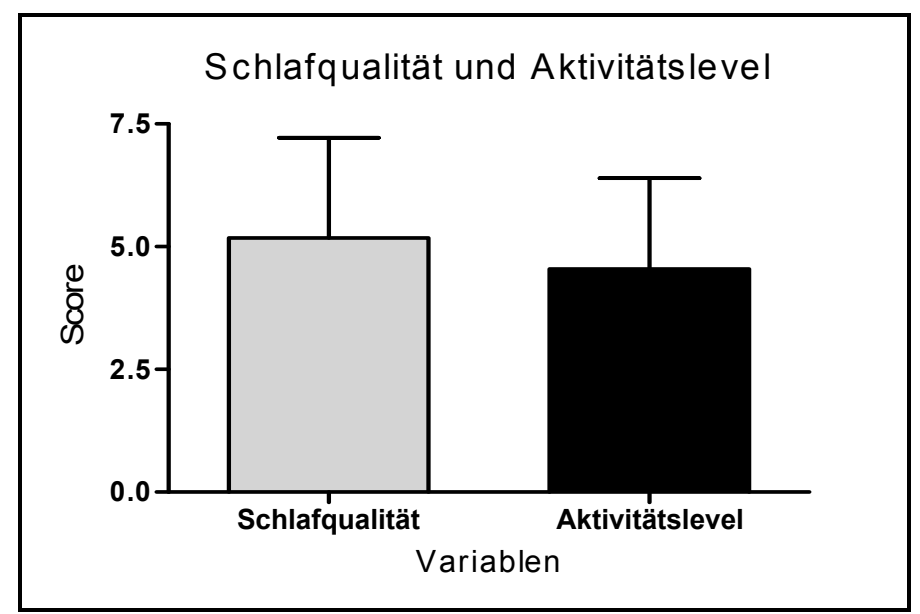

Abbildung 19: Schlafqualität und Aktivitätslevel Pre-Test 


\subsection{Ergebnisse der Studie}

\subsubsection{Beschreibung der Stichprobe}

Im Zeitraum der Studiendurchführung füllten in einer Vollerhebung 1089 Patienten den Fatigue-LASA aus.

Die Probandenzahl für die Studie wurde im Vorfeld auf 100 Probanden festgelegt. Nach der Informationsveranstaltung zur Studie nahmen 101 Patienten am Messzeitpunkt T1 teil. Von diesen waren zu Beginn 56 der Kontroll- und 45 der Trainingsgruppe zugeteilt. Zum Messzeitpunkt T2 hatte sich die Anzahl durch fehlende Unterlagen oder Zurücknahme der Einwilligungserklärung auf 78 reduziert. Davon waren 43 Probanden in der Kontroll- und 35 Probanden in der Trainingsgruppe. An der gesamten Studie nahmen letztendlich 66 Patienten teil, die zu allen drei Messzeitpunkten die erforderlichen Messverfahren vollständig durchliefen.

Von diesen Patienten waren 35 der Kontroll- und 31 der Trainingsgruppe zugeteilt.

Patientendaten, deren Testunterlagen zu einem Messzeitpunkt nicht vollständig waren, gingen zu den anderen Messzeitpunkten trotzdem vollständig in die Auswertung mit ein, da deren Einverständniserklärung trotzdem vorlag. 


\subsubsection{Fatigue-LASA}

Während des gesamten Zeitraums der Datenerhebung wurden in der Vollerhebung des Fatigue-LASA 1324 Bögen registriert. Dabei lag der Mittelwert bei 4,97士2,4.

In der Auswertung des Fatigue-LASA wurden Kategorien gebildet, um die Ergebnisse der LASA Skala zu unterteilen und besser bewerten zu können. Diese sind in Abbildung 20 grafisch dargestellt. Die Verteilung der Menge wurde absolut und in Prozent in Tabelle 3 angegeben.

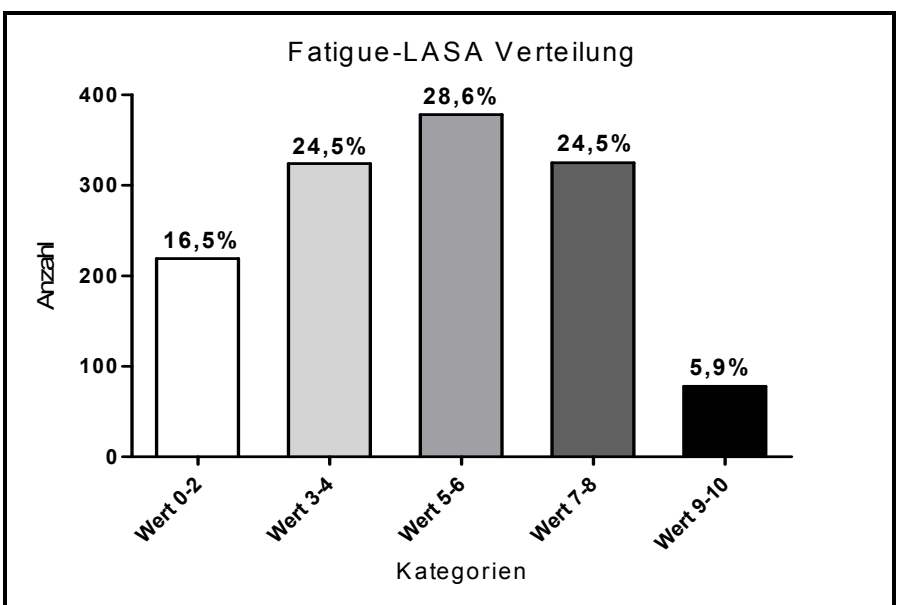

Abbildung 20: Fatigue-LASA Verteilung

\begin{tabular}{|l|r|r|r|r|r|}
\hline Fatigue-LASA & \multicolumn{1}{|c|}{$\mathbf{0 - 2}$} & \multicolumn{1}{|c|}{$\mathbf{3 - 4}$} & \multicolumn{1}{|c|}{$\mathbf{5 - 6}$} & \multicolumn{1}{c|}{$\mathbf{7 - 8}$} & \multicolumn{1}{c|}{$\mathbf{9 - 1 0}$} \\
\hline Anzahl absolut & 219 & 324 & 378 & 325 & 78 \\
\hline Prozent & $16,5 \%$ & $24,5 \%$ & $28,6 \%$ & $24,5 \%$ & $5,9 \%$ \\
\hline
\end{tabular}

Tabelle 3: Fatigue-LASA Verteilung

\subsubsection{Soziodemographische Daten}

Zur Auswertung der Soziodemographischen Daten wurden die Ergebnisse des Fragebogens T1 statistisch ausgewertet. Hierzu muss angemerkt werden, dass 54 Probanden der Kontrollgruppe und 41 Probanden der Trainingsgruppe diesen Fragebogen vollständig ausgefüllt abgegeben haben. Die Statistik des Fragebogens T1 nimmt diese Zahlen als Grundmenge an. Sollte sich die Grundmenge durch 
Nichtbeantwortung einzelner Probanden ändern, wird im jeweiligen Absatz der Statistik eine Anmerkung gemacht und auf die neue Grundmenge hingewiesen.

\section{Altersstruktur/-verteilung}

Der Altersdurchschnitt aller Probanden lag bei 53,4 Jahren. Die Kontrollgruppe war im Mittel 54,7 Jahre und die Trainingsgruppe 51,5 Jahre alt.

Die Altersgruppenverteilung ist Tabelle $4 \mathrm{zu}$ entnehmen. Die Patienten der Trainingsgruppe waren im Durchschnitt etwas jünger.

\begin{tabular}{|l|r|r|r|}
\hline Altersgruppen & $\begin{array}{r}\text { Kontrollgruppe } \\
\text { absolut (in Prozent) }\end{array}$ & $\begin{array}{r}\text { Trainingsgruppe } \\
\text { absolut (in Prozent) }\end{array}$ & $\begin{array}{c}\text { Gesamt } \\
\text { absolut (in Prozent) }\end{array}$ \\
\hline $\mathbf{3 1 - 4 0}$ & $3(5,4 \%)$ & $3(6,7 \%)$ & $6(5,9 \%)$ \\
\hline $\mathbf{4 1 - 5 0}$ & $14(25,0 \%)$ & $20(44,4 \%)$ & $34(33,6 \%)$ \\
\hline $\mathbf{5 1 - 6 0}$ & $26(46,4 \%)$ & $15(33,3 \%)$ & $41(40,6 \%)$ \\
\hline $\mathbf{6 1 - 7 5}$ & $13(23,2 \%)$ & $7(15,6 \%)$ & $20(19.9 \%)$ \\
\hline Gesamt & 56 & 45 & 101 \\
\hline
\end{tabular}

Tabelle 4: Altersgruppenverteilung

Geschlecht

Aus Tabelle 5 wird ersichtlich, dass in beiden Gruppen der Großteil der Probanden weiblich war. Mit 14,3\% in der Kontrollgruppe und 8,9\% in der Trainingsgruppe war das männliche Geschlecht in beiden Gruppen unterrepräsentiert.

\begin{tabular}{|l|r|r|r|}
\hline Geschlecht & $\begin{array}{c}\text { Kontrollgruppe } \\
\text { absolut (in Prozent) }\end{array}$ & $\begin{array}{c}\text { Trainingsgruppe } \\
\text { absolut (in Prozent) }\end{array}$ & $\begin{array}{c}\text { Gesamt } \\
\text { absolut (in Prozent) }\end{array}$ \\
\hline Männlich & $8(14,3 \%)$ & $4(8,9 \%)$ & $12(11,9 \%)$ \\
\hline Weiblich & $48(85,7 \%)$ & $41(91,1 \%)$ & $89(88,1 \%)$ \\
\hline Gesamt & 56 & 45 & 101 \\
\hline
\end{tabular}

Tabelle 5: Geschlechterverteilung 
Berufliche Situation

Abbildung 21 zeigt die berufliche Situation beider Gruppen. Der Anteil an Rentnern fiel in der Trainingsgruppe (12,2\%) geringer aus als in der Kontrollgruppe mit 34\%. Daraus resultierte ein höherer Anteil an Ganztagsbeschäftigung der Trainingsgruppe $(46,3 \%)$. Die Verteilung auf die übrigen Kategorien war ähnlich.

Kontrollgruppe

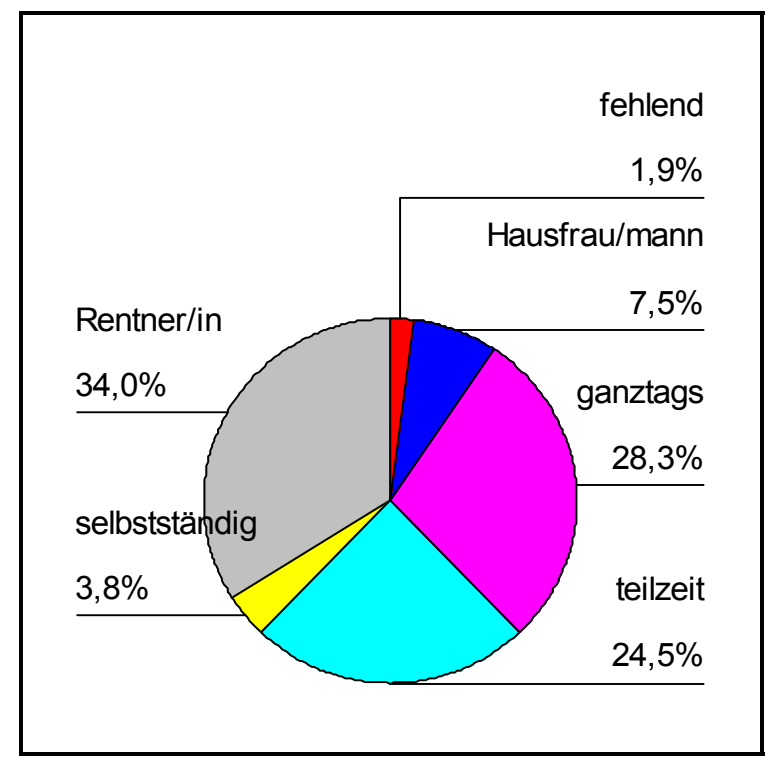

Trainingsgruppe

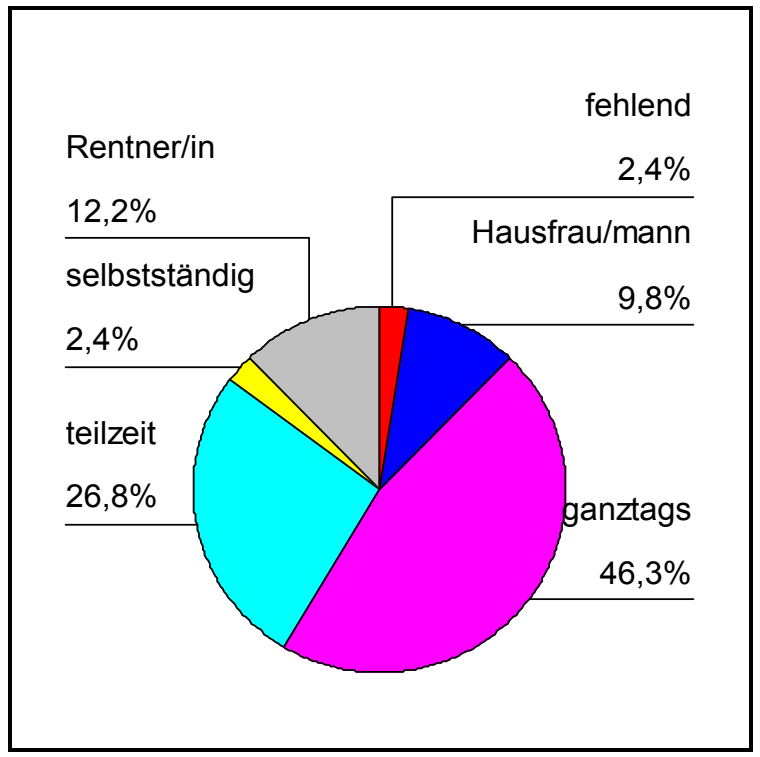

Abbildung 21: Berufliche Situation Kontrollgruppe (links) und Trainingsgruppe (rechts)

\subsubsection{Anthropometrische Daten}

Die Durchschnittsgröße der Probanden lag insgesamt bei $1,67 \mathrm{~m}$ bei einem Durchschnittsgewicht von $74,9 \mathrm{~kg}$. Größe und Gewicht unterschieden sich zwischen den Gruppen kaum. Die Mittelwerte waren annähernd gleich.

Der BMI (Body-Mass-Index) wurde in dieser Arbeit als charakterisierender Faktor für Übergewicht gesehen, da eine Körperfettmessung mittels Kalipometrie aufgrund der fehlenden Technik nicht durchgeführt werden konnte. 
Eine Klassifizierung des BMI findet sich in der Tabelle ${ }^{1}$. Durchschnittlich hatten die weiblichen Probanden beider Gruppen einen BMI von 26,4. Im Mittel lagen männliche Probanden geringfügig darüber und hatten einen BMI von 26,6. Die Unterschiede der Mittelwerte zwischen Kontroll- und Trainingsgruppe waren marginal und wurden nicht weiter betrachtet. Allerdings konnte, wie in Tabelle 6 dargestellt, ein höherer Anteil an Übergewichtigen in der Trainingsgruppe beobachtet werden.

Letztlich war nur ein Anteil von 32,6\% der gesamten Stichprobe im Bereich Normalgewicht. Ansonsten konnte ein hoher Anteil der Probanden in den Bereichen Übergewicht, Adipositas und Massive Adipositas festgestellt werden. Zusammenhänge zwischen erhöhtem BMI und Bewegungsmangel in Verbindung mit falscher Ernährung sind anzunehmen.

\begin{tabular}{|l|r|r|r|r|r|r|}
\hline $\begin{array}{l}\text { Klassifikation } \\
\text { BMI }\end{array}$ & $\begin{array}{r}\text { Kontrolle } \\
\text { Kontrolle }\end{array}$ & $\begin{array}{r}\text { Kontrolle } \\
\text { Gesamt }\end{array}$ & $\begin{array}{r}\text { Training } \\
\text { Training }\end{array}$ & $\begin{array}{r}\text { Training } \\
\text { Gesamt }\end{array}$ \\
\hline Untergewicht & 0 & 0 & 0 & 1 & 0 & 1 \\
& & & & & & \\
\hline Normalgewicht & 15 & 3 & 18 & 9 & 1 & 10 \\
& & & $(38,3 \%)$ & & & $(25,6 \%)$ \\
\hline Übergewicht & 17 & 4 & 21 & 19 & 3 & 22 \\
& & & $(44,7 \%)$ & & & $(56,4 \%)$ \\
\hline Adipositas & 6 & 1 & 7 & 5 & 0 & 5 \\
& & & $(14,9 \%)$ & & & $(12,8 \%)$ \\
\hline Massive & 1 & 0 & 1 & 1 & 0 & 1 \\
Adipositas & & & $(2,1 \%)$ & & & $(2,6 \%)$ \\
\hline
\end{tabular}

Tabelle 6: Klassifikation BMI im Probandenkollektiv

\begin{tabular}{|l|r|r|}
\hline Klassifizierung BMI & \multicolumn{1}{|c|}{ männlich } & \multicolumn{1}{|l|}{ Weiblich } \\
\hline Untergewicht & $<20$ & $<19$ \\
\hline Normalgewicht & $20-25$ & $19-24$ \\
\hline Übergewicht & $25-30$ & $24-30$ \\
\hline Adipositas & $30-40$ & $30-40$ \\
\hline Massive Addipositas & $>40$ & $>40$ \\
\hline
\end{tabular}

Tabelle: Klassifizierung BMI (HOLLMANN \& HETTINGER 2000) 


\subsubsection{Allgemeine Daten zur Erkrankung}

Diagnosen

Abbildung 22 zeigt die Diagnosen des gesamten Probandenkollektivs. Es wurden die fünf am meist vertretenen Tumorarten abgebildet. Nur selten vorgekommene Tumorarten wurden unter „andere“ zusammengefasst. Das Mammakarzinom ist mit über $50 \%$ am stärksten repräsentiert. Weitere erfasste Tumorarten waren maligne Lymphome, maligne Darmtumore, Ovarialkarzinome und Cervixkarzinome.

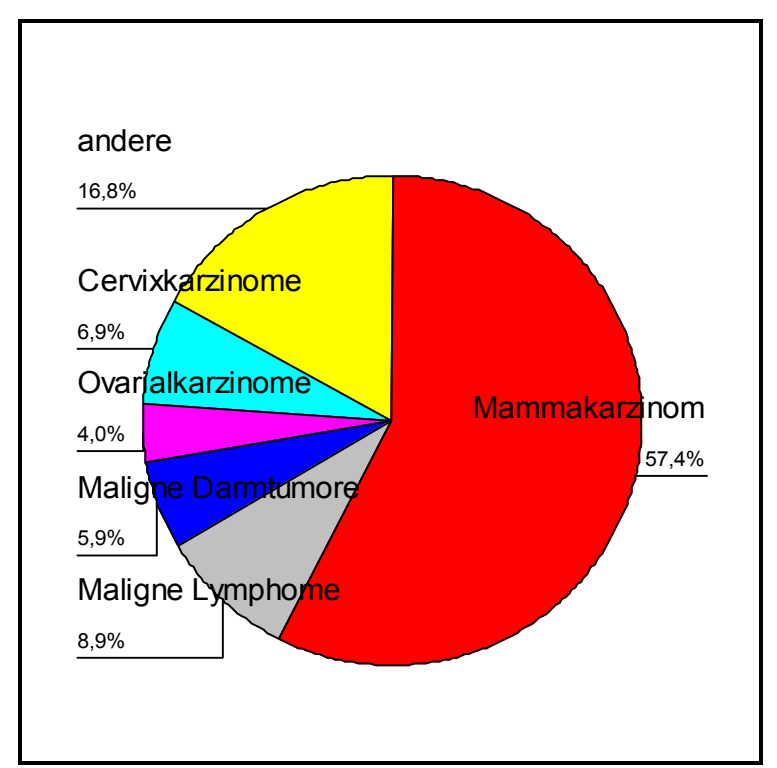

Abbildung 22: Diagnosen des gesamten Patientenkollektivs 
In Abbildung 23 ist eine Aufteilung in Kontroll- und Trainingsgruppe abgebildet. Die Darstellungsweise ist identisch zu Abbildung 22.

Kontrollgruppe

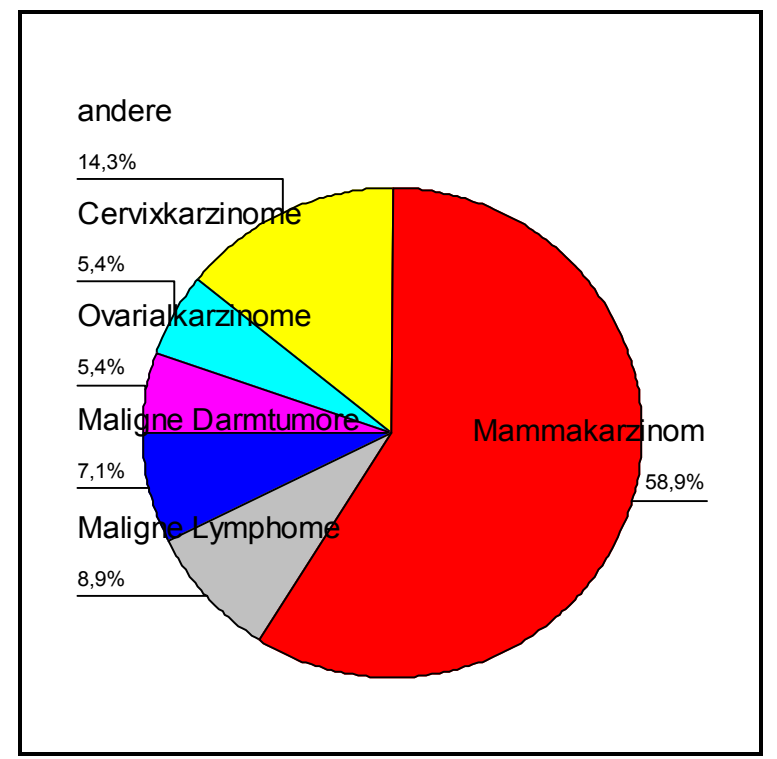

Abbildung 23: Diagnosen Kontrollgruppe (links) und Trainingsgruppe (rechts)
Trainingsgruppe

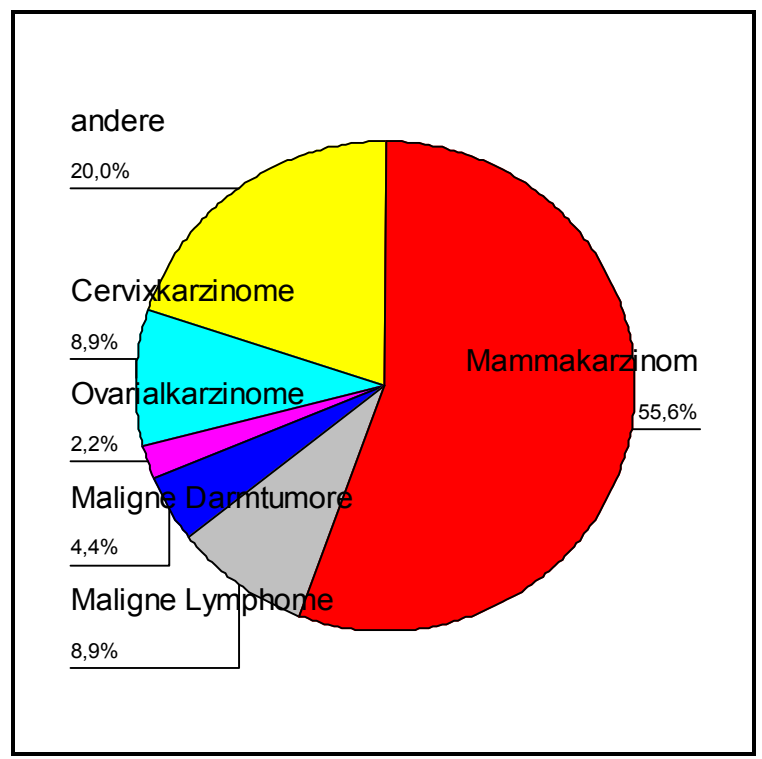

Tabelle 7 zeigt die exakten absoluten und prozentualen Werte der Diagnosen des gesamten Probandenkollektivs sowie die der Kontroll- und Trainingsgruppe.

\begin{tabular}{|l|r|r|r|}
\hline Diagnose & Kontrollgruppe & Trainingsgruppe & \multicolumn{1}{c|}{ Gesamt } \\
\hline Mammakarzinom & $33(58,9 \%)$ & $25(55,6 \%)$ & $58(57,4 \%)$ \\
\hline Mal. Lymphome & $5(8,9 \%)$ & $4(8,9 \%)$ & $9(8,9 \%)$ \\
\hline Mal. Darmtumore & $4(7,1 \%)$ & $2(4,4 \%)$ & $6(5,9 \%)$ \\
\hline Ovalkarzinome & $3(5,4 \%)$ & $1(2,2 \%)$ & $4(4,0 \%)$ \\
\hline Cervixkarzinome & $3(5,4 \%)$ & $4(8,9 \%)$ & $7(6,9 \%)$ \\
\hline Andere & $8(14,3 \%)$ & $9(20,0 \%)$ & $17(16,8 \%)$ \\
\hline
\end{tabular}

Tabelle 7: Diagnosen 
Zeitraum der Diagnose

In der Abbildung 24 ist der Zeitraum der Erstdiagnose beider Gruppen zu sehen. Es wird deutlich, dass in der Trainingsgruppe mehr Probanden die Diagnose innerhalb der letzten 6 Monate bekommen hat als in der Kontrollgruppe. In beiden Gruppen ist erkennbar, dass die Fatigueerkrankung nicht ausschließlich in den ersten Monaten nach Diagnose auftrat, sondern bei einem Teil der Patienten einen chronischen Verlauf nahm und auch nach mehreren Jahren noch einen einschränkenden Charakter hatte.

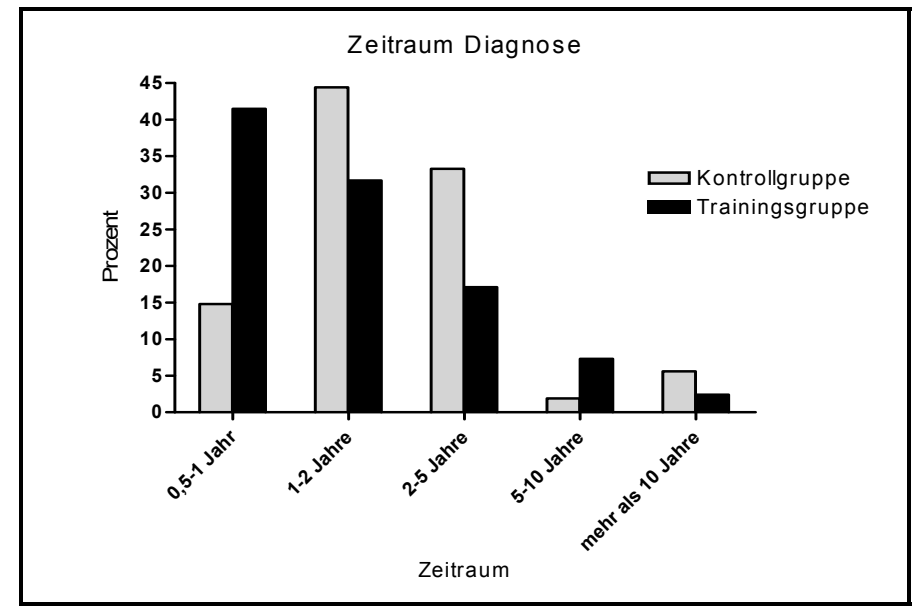

Abbildung 24: Zeitraum Diagnose

\section{Art der Erstbehandlung}

Tabelle 8 zeigt, dass in beiden Gruppen ein Anteil von über 50 Prozent der Patienten mit einer Kombination aus verschiedenen Therapien behandelt wurde.

Die Hormontherapie kam als Einzeltherapie weder in der Kontroll- noch in der Trainingsgruppe zur Anwendung. Während in der Kontrollgruppe ein Anteil von $11,1 \%$ allein durch Bestrahlung behandelt wurde, kam diese Behandlungsform in der Trainingsgruppe nicht vor. 


\begin{tabular}{|l|r|r|r|}
\hline $\begin{array}{l}\text { Art der } \\
\text { Erstbehandlung }\end{array}$ & Kontrollgruppe & Trainingsgruppe & Gesamt \\
\hline Operation & $9(16,7 \%)$ & $14(35 \%)$ & $23(24,5 \%)$ \\
\hline Strahlentherapie & $3(5,5 \%)$ & 0 & $3(3,2 \%)$ \\
\hline Chemotherapie & $6(11,1 \%)$ & $2(5 \%)$ & $8(8,5 \%)$ \\
\hline Hormontherapie & 0 & 0 & 0 \\
\hline Kombinationstherapie & $36(66,7 \%)$ & $24(60 \%)$ & $60(63,8 \%)$ \\
\hline Gesamt & 54 & 40 & 94 \\
\hline
\end{tabular}

Tabelle 8: Art der Erstbehandlung

Momentane Behandlungen

In der Kontrollgruppe unterzog sich zum Zeitpunkt der Studie ein Anteil von 42,6\% einer Therapie. Es handelte sich bei ausschließlich um Hormon- oder Antikörpertherapien.

In der Trainingsgruppe ergab sich ein ähnliches Bild. Allerdings befand sich noch ein Anteil von $63,4 \%$ in einer laufenden Therapie. Es handelte sich aber auch hier ausschließlich um Hormon- und Antikörpertherapien. 


\subsubsection{Allgemeine Daten zu sportlichen Vorerfahrungen}

\section{Sportliche Aktivität vor und während der Erkrankung}

Abbildung 25 vergleicht die Verteilung der sportlichen Aktivität vor der Erkrankung und vor der Rehabilitation in der Kontrollgruppe. Die deutliche Erhöhung der Zahl der Patienten, die vor der Erkrankung regelmäßig sportlich aktiv waren, war angesichts der Tatsache, dass bei ca. 50\% der Patienten erst in den letzten zwei Jahren eine Tumorerkrankung diagnostiziert und anschließend behandelt wurde, nicht verwunderlich. Der Anteil der unregelmäßig sportlich Aktiven vor der Erkrankung war nur $1,9 \%$ höher als vor der Rehabilitation. Dafür war die Zahl der nicht sportlich Aktiven geringfügig niedriger.

Auffallend war, dass die Zahl der Probanden, die zur sportlichen Aktivität vor der Rehabilitation keine Angaben machten, mit 1,9\% vor der Erkrankung auf 20,4\% vor der Rehabilitation deutlich angestiegen ist.

Kontrollgruppe

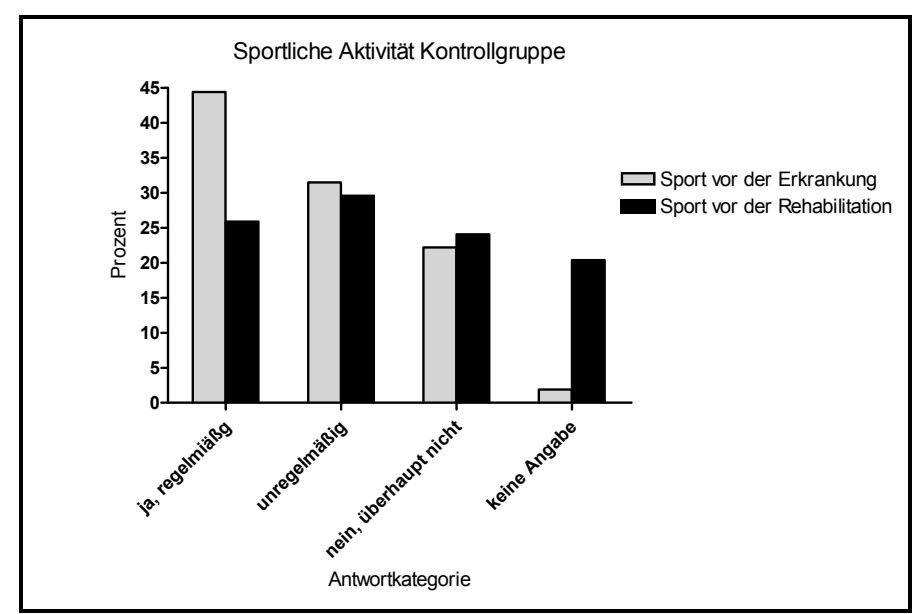

Abbildung 25: Sportliche Aktivität Kontrollgruppe

In der Trainingsgruppe zeigt sich in Abbildung 26 ein etwas anderes Bild als in der Kontrollgruppe.

Während der Anteil der regelmäßig sportlich Aktiven vor der Erkrankung bei nahezu $50 \%$ lag und auch vor der Rehabilitation nicht entscheidend absank, fiel der Anteil der unregelmäßig sportlich Aktiven von 41,5\% vor der Erkrankung auf $19,5 \%$ vor der 
Rehabilitation. Allerdings stieg der Anteil der nicht sportlich Aktiven in der Trainingsgruppe von $9,8 \%$ auf $34,1 \%$ an.

In der Trainingsgruppe wurden von allen Probanden Angaben zur sportlichen Aktivität gemacht.

Trainingsgruppe

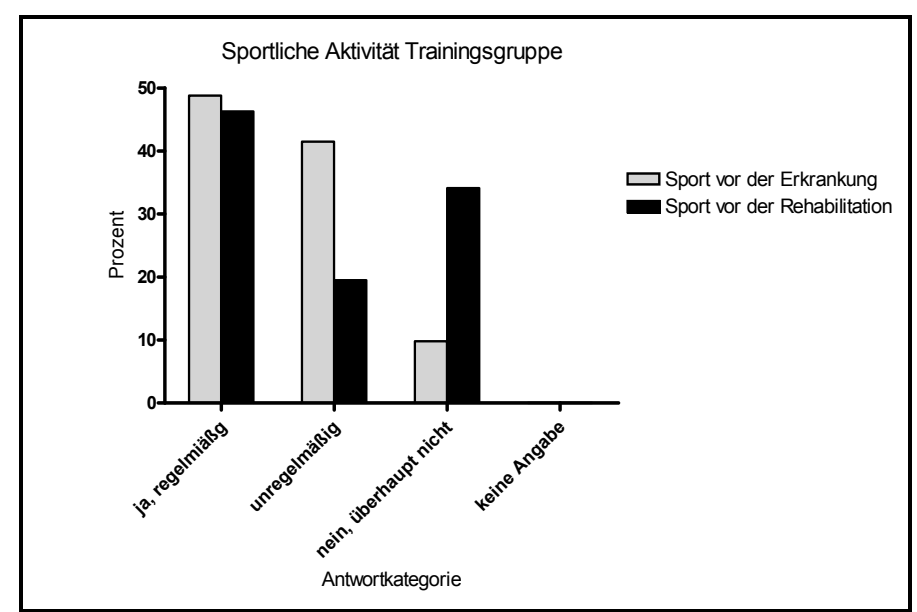

Abbildung 26: Sportliche Aktivität Trainingsgruppe

\section{Sportkategorien}

Um eine Übersichtlichkeit zu gewährleisten, wurden alle genannten Sportarten in drei Kategorien eingeordnet. Sportarten wie Nordic Walking, Schwimmen oder Radfahren, die primär die Ausdauer trainieren, wurden in der Kategorie Ausdauersportarten zusammengefasst. Fitnesskurse, Yoga, Gerätetraining oder Gymnastik wurden in die Kategorie Fitness eingeordnet. Die letzte Kategorie waren die Spielsportarten, in der Sportarten wie Fußball, Volleyball, Tischtennis oder Tennis zusammengefasst wurden. Mehrfachnennungen im Fragebogen waren möglich. Es wurde allerdings immer nur eine Sportart pro Kategorie gewertet. Falls also ein Proband Nordic Walking, Schwimmen und Radfahren betrieb, wurde dies nur einmal in der Kategorie Ausdauersport gewertet. Betrieb ein Proband allerdings Nordic Walking und Yoga, wurden diese einmal in der Kategorie Ausdauersport und einmal in der Kategorie Fitness gewertet. 
In Abbildung 27 ist die Verteilung der Sportkategorien vor der Erkrankung und vor der Rehabilitation beider Gruppen zu sehen.

Die Beantwortungsquote dieser Frage war sehr unterschiedlich. Während $87 \%$ der Kontrollgruppe die Frage nach der Art der sportlichen Aktivität vor der Erkrankung beantwortet haben, waren es bei der Trainingsgruppe nur 67\%.

Es ist erkennbar, dass sowohl bei der Kontroll- als auch bei der Trainingsgruppe Ausdauersport die am höchsten besetzte Kategorie war.

In der Kategorie Ausdauersport wies die Kontrollgruppe zum Zeitpunkt vor der Erkrankung mit 52,1\% einen niedrigeren Wert auf als die Trainingsgruppe mit 59,6\%. Die Kategorie Fitness war mit $43,8 \%$ in der Kontrollgruppe höher repräsentiert als in der Trainingsgruppe mit 34,0\%.

Die Kategorie Spielsportarten war zu jedem Zeitpunkt mit unter 10\% unterrepräsentiert.

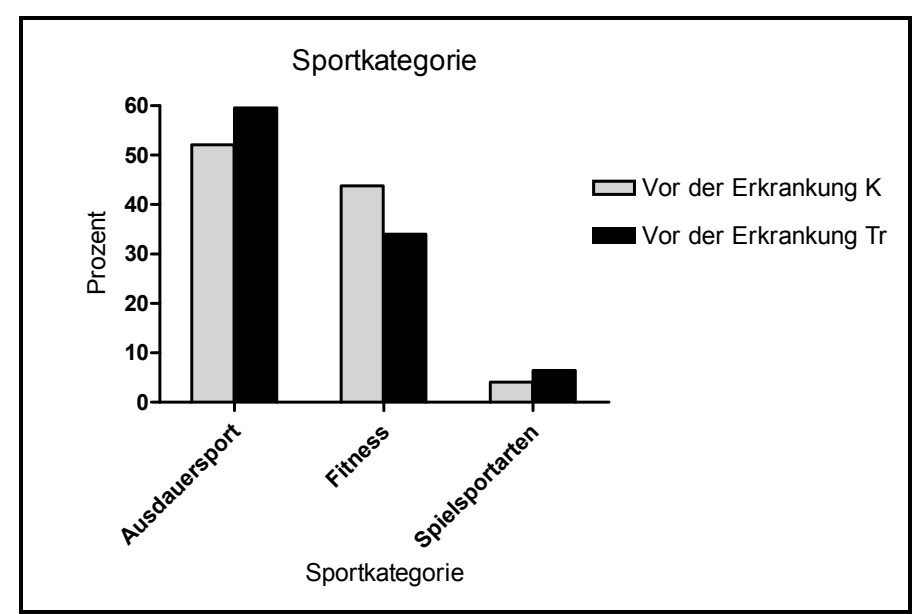

Abbildung 27: Sportkategorie vor der Erkrankung

Bei der Frage nach der Art der sportlichen Aktivität vor der Rehabilitation sank die Antwortquote in der Kontrollgruppe auf 55,6\% und in der Trainingsgruppe auf $61,5 \%$. Abbildung 28 zeigt die Sportkategorie vor der Rehabilitation.

Die Kategorie Ausdauersport war mit etwa $56 \%$ in beiden Gruppen nahezu ausgeglichen. 
Die Kontrollgruppe wies in der Fitnesskategorie einen Wert von 36,6\% und die Trainingsgruppe einen Wert von $43,7 \%$ auf. Spielsportarten waren erneut unterrepräsentiert.

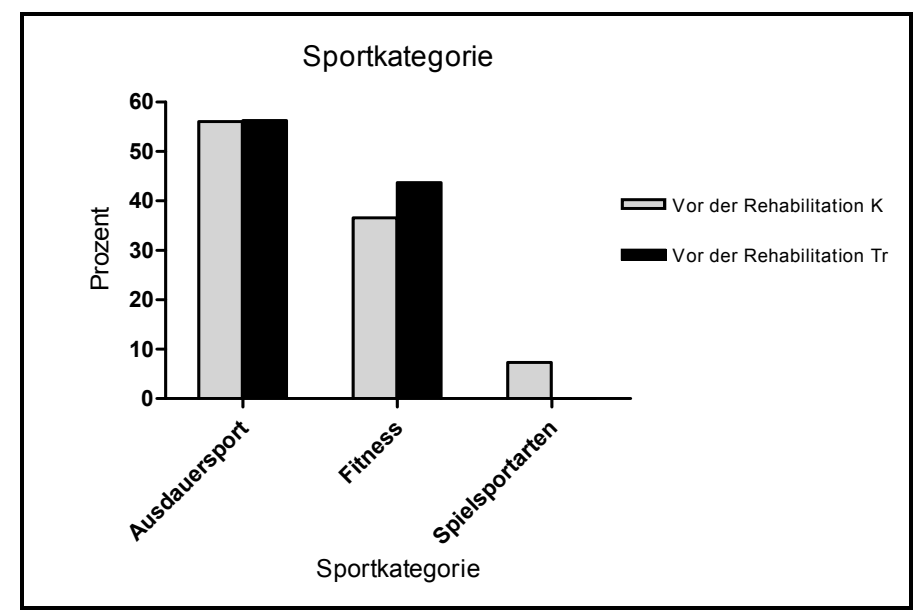

Abbildung 28: Sportkategorie vor der Rehabilitation

\section{Zeitlicher Aufwand}

Im Vorfeld muss angemerkt werden, dass die Frage nach dem zeitlichen Aufwand zur sportlichen Aktivität zum Zeitpunkt vor der Erkrankung in der Kontrollgruppe von 40 Probanden und in der Trainingsgruppe von lediglich 31 Probanden beantwortet wurde. Zum Zeitpunkt vor der Rehabilitation waren es in der Kontrollgruppe noch 30 und in der Trainingsgruppe noch 25 Probanden. Gründe für die geringe Beantwortung waren vor allen Dingen, die schon im oberen Abschnitt erwähnte generelle Einstellung zu Sport im Allgemeinen.

Abbildung 29 zeigt, wie hoch der zeitliche Aufwand beider Gruppen für sportliche Aktivitäten zu den Zeitpunkten vor der Erkrankung und vor der Rehabilitation war. Zu beiden Zeitpunkten war festzustellen, dass der zeitliche Aufwand sowohl in der Kontroll- als auch in der Trainingsgruppe ähnlich war. 
Vom Messzeitpunkt vor der Erkrankung zum Messzeitpunkt vor der Rehabilitation war ein deutlicher Anstieg im Bereich von 1-2 Stunden festzustellen. Im Bereich 3-4 Stunden hingegen fiel die Anzahl der Nennungen. Im Übrigen handelt es sich nur um geringe Änderungen im zeitlichen Aufwand.
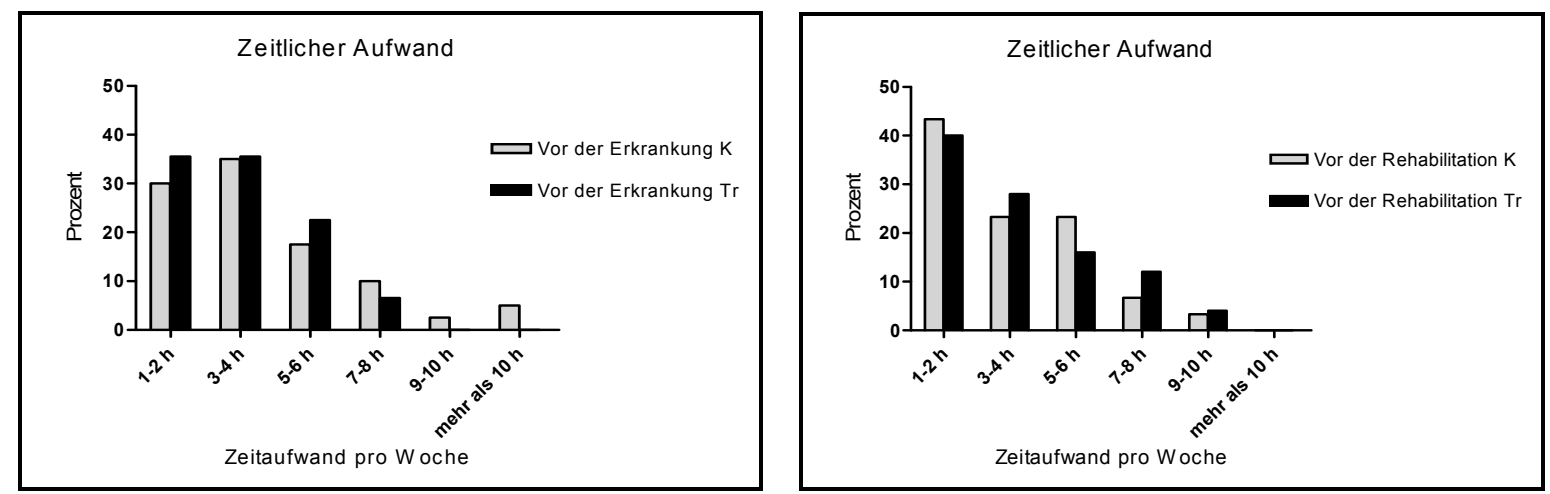

Abbildung 29: Zeitlicher Aufwand vor Erkrankung (links) und vor Rehabilitation (rechts)

Sportliche Aktivität während der Rehabilitation

Während der Rehabilitation führten beide Gruppen der Studie das Bewegungs- und Sportprogramm der Sonnenberg-Klinik durch. Dieses bestand aus verschiedensten Bewegungsangeboten in Bereichen des Ausdauersports (bspw. Fahrradergometrie, Nordic Walking, Schwimmen), der Kraft und Fitness (bspw. Medizinische Trainingstherapie, Sportgruppentherapie) und der Physiotherapie (bspw. Einzelkrankengymnastik, Mobilisationsgruppentherapie, Bewegungsbad). Alle Patienten bekamen, angepasst an den persönlichen Gesundheits- und Fitnesszustand, gezielte Therapiepläne, in denen sportliche Aktivität zum Teil mit integriert war. Zusätzlich waren die Patienten herzlich dazu eingeladen, die Sportstätten und Angebote der Klinik in den freien Zeiten ihres Therapieplanes zu nutzen. Patienten der Trainingsgruppe waren zusätzlich zu ihrem Therapieplan angehalten, zwei- bis dreimal pro Woche das Trainingsprogramm durchzuführen. 


\section{Sportmotive}

Die Motivation der Patienten war in beiden Gruppen sehr ähnlich. In Abbildung 30 ist erkennbar, dass Gesundheit als Motiv zu allen Messzeitpunkten eine hohe Wertigkeit einnahm. Während die anderen Motive fortschreitend abnahmen, stieg das Gesundheitsmotiv an.
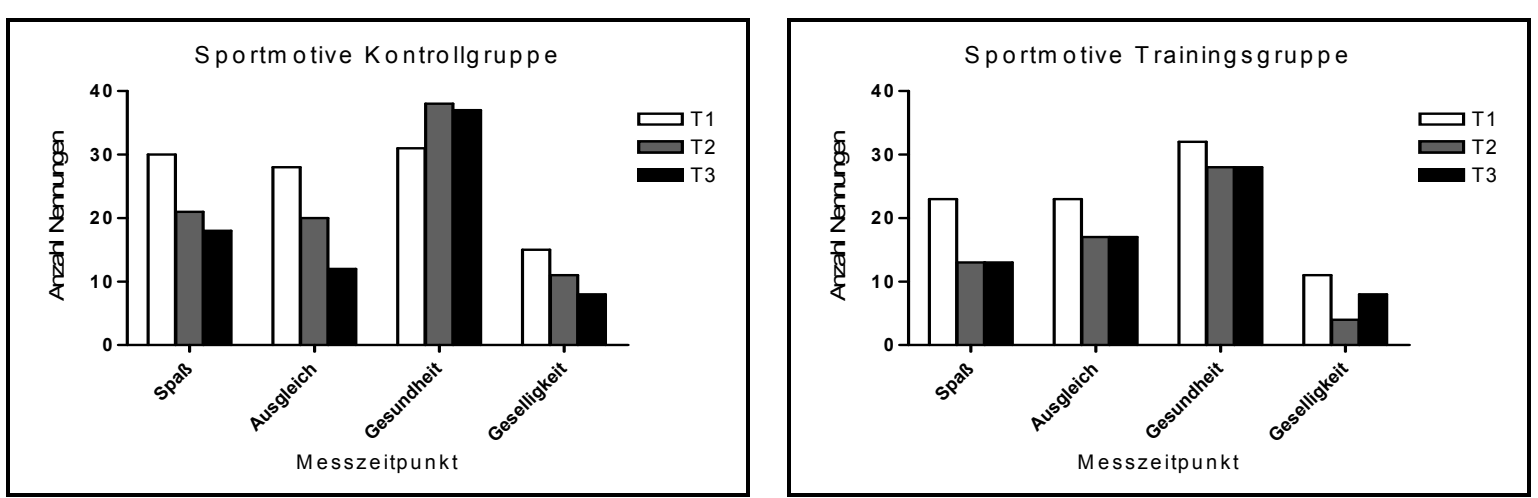

Abbildung 30: Sportmotive von Kontrollgruppe (links) und Trainingsgruppe (rechts) 
Subjektive Selbsteinschätzung der körperlichen Leistungsfähigkeit

Im Rahmen des Dokumentationsbogens wurden die Patienten gebeten, ihre körperliche Leistungsfähigkeit subjektiv zu beurteilen. Eine Einschätzung der subjektiven Selbsteinschätzung körperlicher Leistungsfähigkeit konnte durch eine Auswahl zwischen den Antwortmöglichkeiten "sehr gut", "gut", „weniger gut" und „schlecht" getroffen werden. Die prozentualen Ergebnisse finden sich in Abbildung 31. Dabei wurden die Antwortkategorien zu allen drei Messzeitpunkten ausgezählt und prozentual zum Patientenkollektiv dargestellt. Anhand der Abbildung wird deutlich, dass nur geringe Unterschiede zwischen den Gruppen bestanden. In beiden Gruppen fand während der gesamten Studiendauer eine Verschiebung der Selbsteinschätzung in positive Richtung statt.
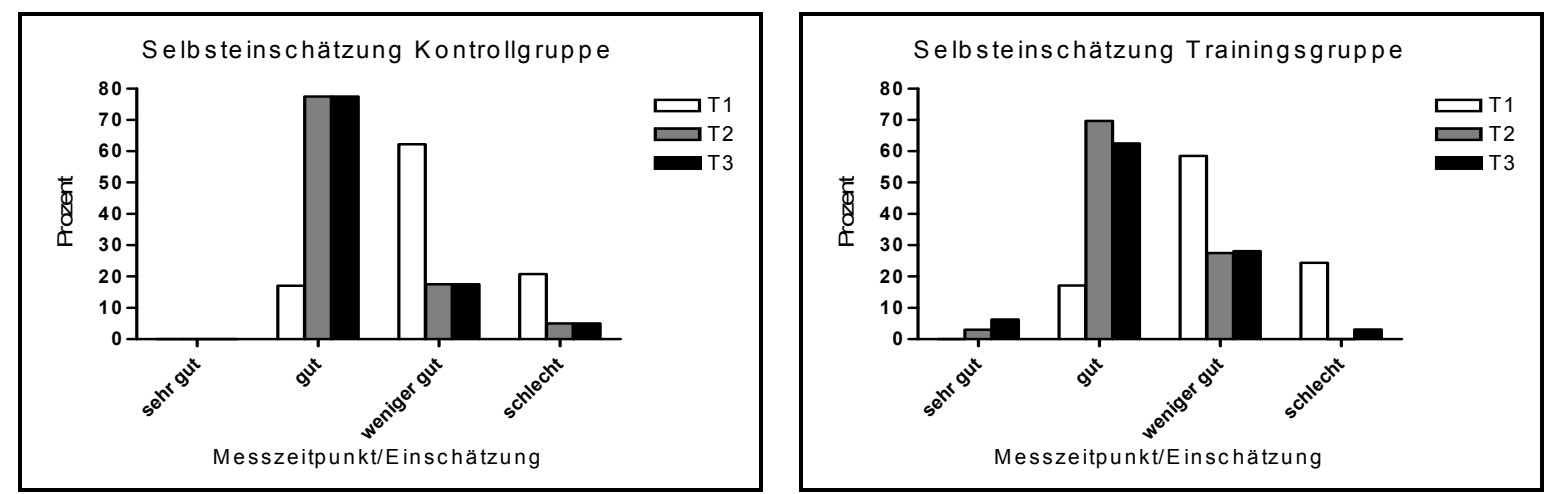

Abbildung 31: Subjektive Selbsteinschätzung Kontrollgruppe (links) und Trainingsgruppe (rechts) 


\subsubsection{Fatiguerhythmik}

Im folgenden Abschnitt werden die Ergebnisse des Fragebogens zur Fatiguerhythmik dargestellt. Die Darstellung wurde in zwei unterschiedlichen Arten vorgenommen. Zum Ersten wurden die Ergebnisse gruppenintern zu allen drei Messzeitpunkten verglichen. Daraus ließen sich Rückschlüsse auf die Entwicklung der Fatiguerhythmik der Patienten ziehen. Weiterführend wurden die Ergebnisse beider Gruppen zu allen drei Messzeitpunkten miteinander verglichen.

\subsubsection{Gruppeninterner Vergleich zu allen drei Messzeitpunkten}

Kontrollgruppe

In Abbildung 32 sind die Ergebnisse des Fragebogens zur Fatiguerhythmik grafisch dargestellt. Es handelt sich bei der Abbildung um eine Gegenüberstellung der Mittelwerte aller drei Messzeitpunkte zu den verschiedenen Tageszeiten. Außer bei der Messung um acht Uhr am Morgen konnten zu keiner Uhrzeit signifikante Unterschiede zwischen den drei Messzeitpunkten nachgewiesen werden. Zu dieser einen Uhrzeit konnten signifikante Unterschiede zwischen T1 und T3 nachgewiesen werden. Zu T2 und T3 sind die Mittelwerte der Fatigue Score nicht signifikant niedriger als zu T1.

Des Weiteren wurde deutlich, dass zu allen drei Messzeitpunkten mit fortschreitender Tageszeit ein Anstieg des Fatiguescore zu beobachten war. Die genauen Werte sind als Mittelwerte \pm Standardabweichung Tabelle 9 zu entnehmen. Dort sind auch die Signifikanzwerte aller Messungen zu finden. 


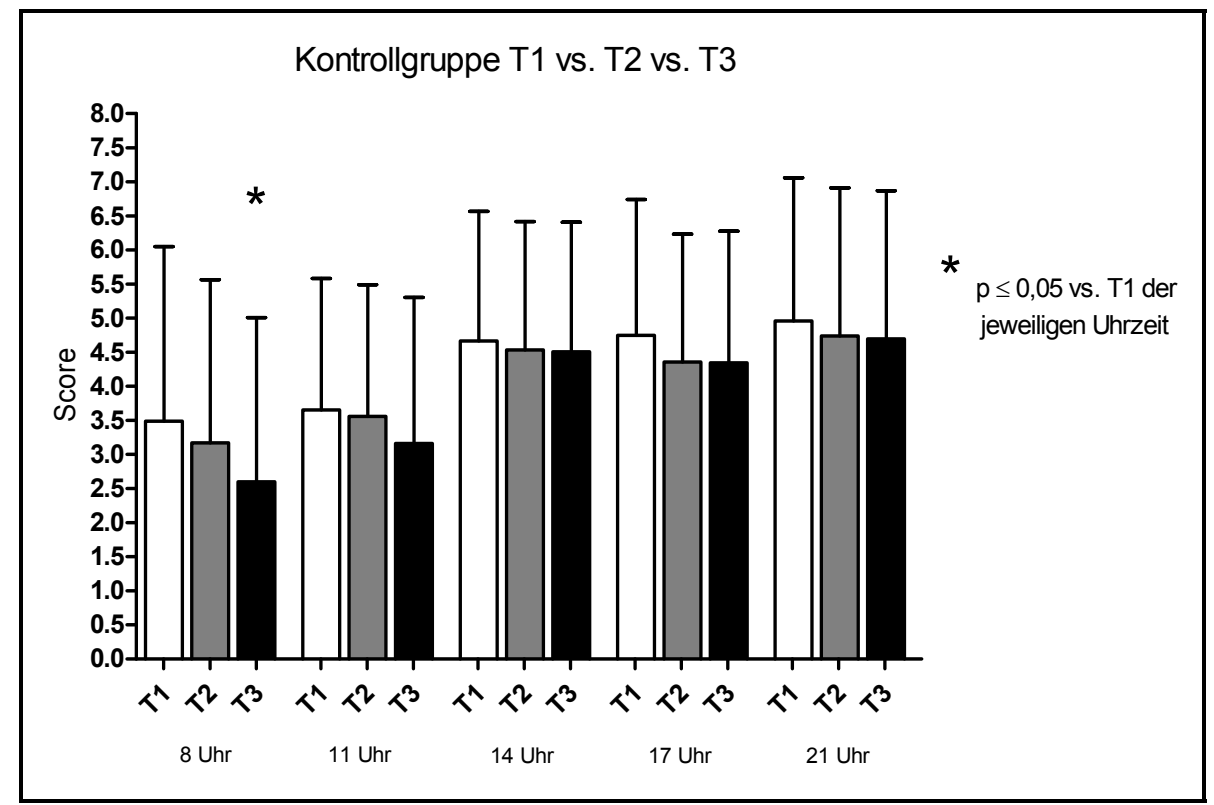

Abbildung 32: Vergleich Tagesrhythmik Fatigue Kontrollgruppe T1 vs. T2 vs. T3

\begin{tabular}{|l|c|c|c|r|r|r|}
\hline Uhrzeit & T1 & T2 & T3 & $\begin{array}{c}\text { Signifikanz } \\
\text { T1 vs. T2 }\end{array}$ & $\begin{array}{r}\text { Signifikanz } \\
\text { T1 vs. T3 }\end{array}$ & $\begin{array}{c}\text { Signifikanz } \\
\text { T2 vs. T3 }\end{array}$ \\
\hline $\mathbf{8 ~ U h r}$ & $3,49 \pm 2,6$ & $3,17 \pm 2,4$ & $2,60 \pm 2,4$ & 0,284 & $\mathbf{0 , 0 0 5}$ & 0,072 \\
\hline $\mathbf{1 1} \mathbf{~ h r}$ & $3,65 \pm 1,9$ & $3,56 \pm 1,9$ & $3,16 \pm 2,1$ & 0,682 & 0,054 & 0,139 \\
\hline $\mathbf{1 4} \mathbf{~ h r}$ & $4,67 \pm 1,9$ & $4,54 \pm 1,9$ & $4,50 \pm 1,9$ & 0,564 & 0,505 & 0,904 \\
\hline $\mathbf{1 7} \mathbf{~ U h r}$ & $4,75 \pm 2,0$ & $4,36 \pm 1,9$ & $4,34 \pm 1,9$ & 0,091 & 0,104 & 0,951 \\
\hline $\mathbf{2 1}$ Uhr & $4,96 \pm 2,1$ & $4,74 \pm 2,2$ & $4,70 \pm 2,2$ & 0,388 & 0,333 & 0,885 \\
\hline
\end{tabular}

Tabelle 9: Vergleich Tagesrhythmik Fatigue Kontrollgruppe T1 vs. T2 vs. T3

Trainingsgruppe

Abbildung 33 zeigt die Ergebnisse des Fragebogens zur Fatiguerhythmik der Trainingsgruppe zu allen drei Messzeitpunkten. Die Mittelwerte des Fatiguescore und die Standardabweichung vom Mittelwert sind graphisch dargestellt. In der Trainingsgruppe konnten mit Ausnahme der Uhrzeit 8 Uhr am Morgen zu allen Tageszeiten signifikante Unterschiede zwischen T1 und T2 gegenüber T3 nachgewiesen werden.

Außerdem ließen sich sowohl um $11 \mathrm{Uhr}$ als auch um $14 \mathrm{Uhr}$ signifikante Unterschiede zwischen T2 und T3 nachweisen. Ein Anstieg des Fatiguescore mit 
fortschreitender Tageszeit war auch hier zu beobachten. Die genauen Mittelwerte \pm Standardabweichungen und die Signifikanzwerte können Tabelle 10 entnommen werden.

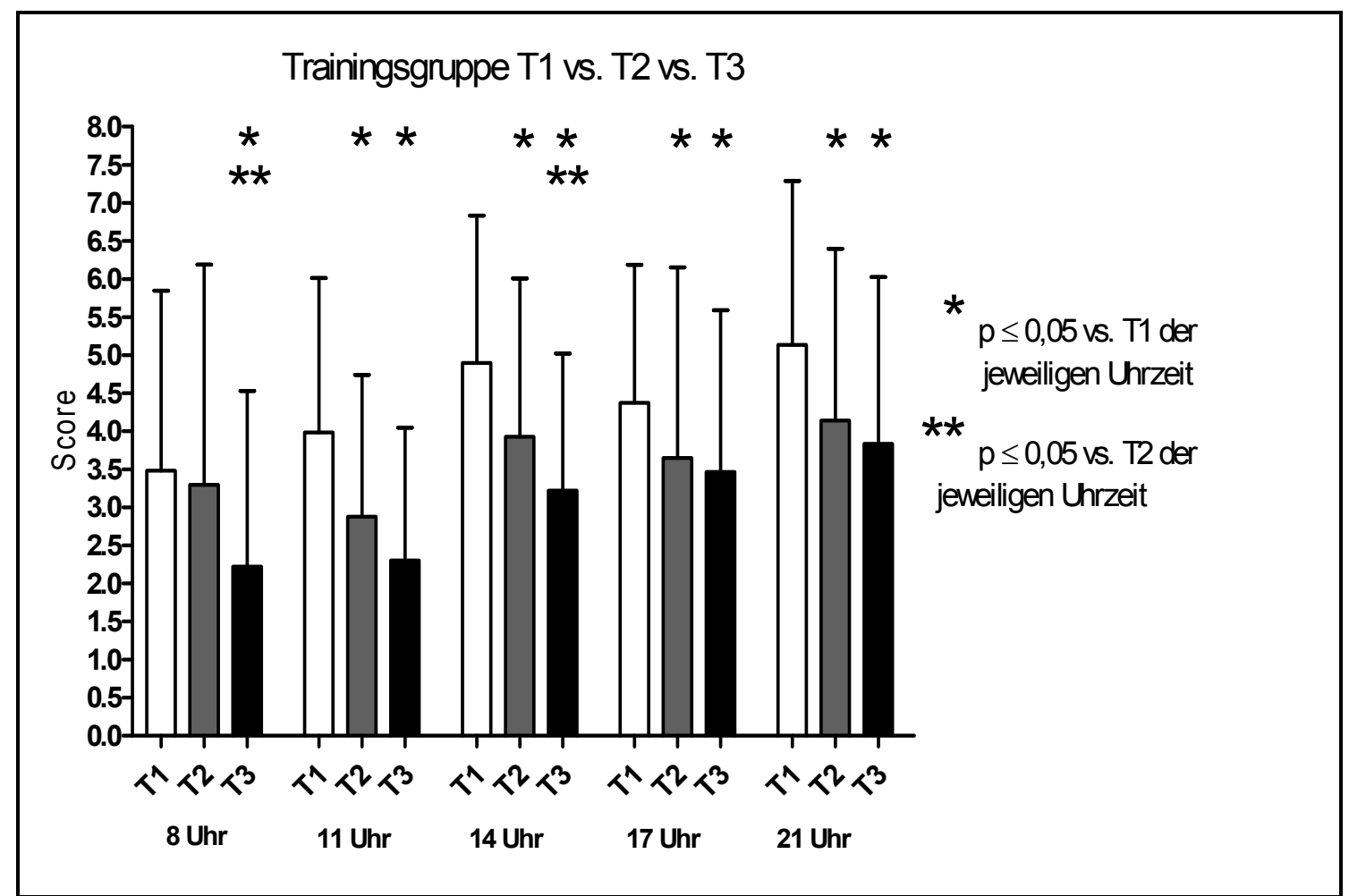

Abbildung 33: Vergleich Tagesrhythmik Fatigue Kontrollgruppe T1 vs. T2 vs. T3

\begin{tabular}{|c|c|c|c|c|c|c|}
\hline Uhrzeit & T1 & T2 & T3 & $\begin{array}{c}\text { Signifikanz } \\
\text { T1 vs. T2 }\end{array}$ & \begin{tabular}{|c|} 
Signifikanz \\
T1 vs. T3
\end{tabular} & $\begin{array}{c}\text { Signifikanz } \\
\text { T2 vs. T3 }\end{array}$ \\
\hline $8 \mathrm{Uhr}$ & $3,48 \pm 2,4$ & $3,30 \pm 2,9$ & $2,22 \pm 2,3$ & 0,6570 & 0,0001 & 0,014 \\
\hline 11 Uhr & $3,98 \pm 2,0$ & $2,88 \pm 1,9$ & $2,30 \pm 1,7$ & 0,0006 & $<0,0001$ & 0,059 \\
\hline $14 \mathrm{Uhr}$ & $4,90 \pm 2,0$ & $3,93 \pm 2,1$ & $3,22 \pm 1,8$ & 0,0003 & $<0,0001$ & 0,030 \\
\hline 17 Uhr & $4,37 \pm 1,8$ & $3,65 \pm 2,5$ & $3,47 \pm 2,1$ & 0,0280 & 0,0009 & 0,637 \\
\hline $21 \mathrm{Uhr}$ & $5,10 \pm 2,1$ & $4,10 \pm 2,3$ & $3,83 \pm 2,2$ & 0,0048 & $<0,0001$ & 0,414 \\
\hline
\end{tabular}

Tabelle 10: Vergleich Tagesrhythmik Fatigue Kontrollgruppe T1 vs. T2 vs. T3 


\subsubsection{Vergleich Kontroll- und Trainingsgruppe zum Messzeitpunkt T1}

Abbildung 34 zeigt einen Vergleich der Fatiguerhythmik beider Gruppen zum Messzeitpunkt T1. Zur genaueren Betrachtung sind in Tabelle 11 die Mittelwerte \pm Standardabweichung inklusive der Signifikanzwerte zwischen Kontroll- und Trainingsgruppe aufgeführt. Allerdings waren zu T1 zu keiner Uhrzeit signifikante Unterschiede zwischen Kontroll- und Trainingsgruppe nachweisbar. Die Ausgangswerte der Gruppen waren annähernd identisch.

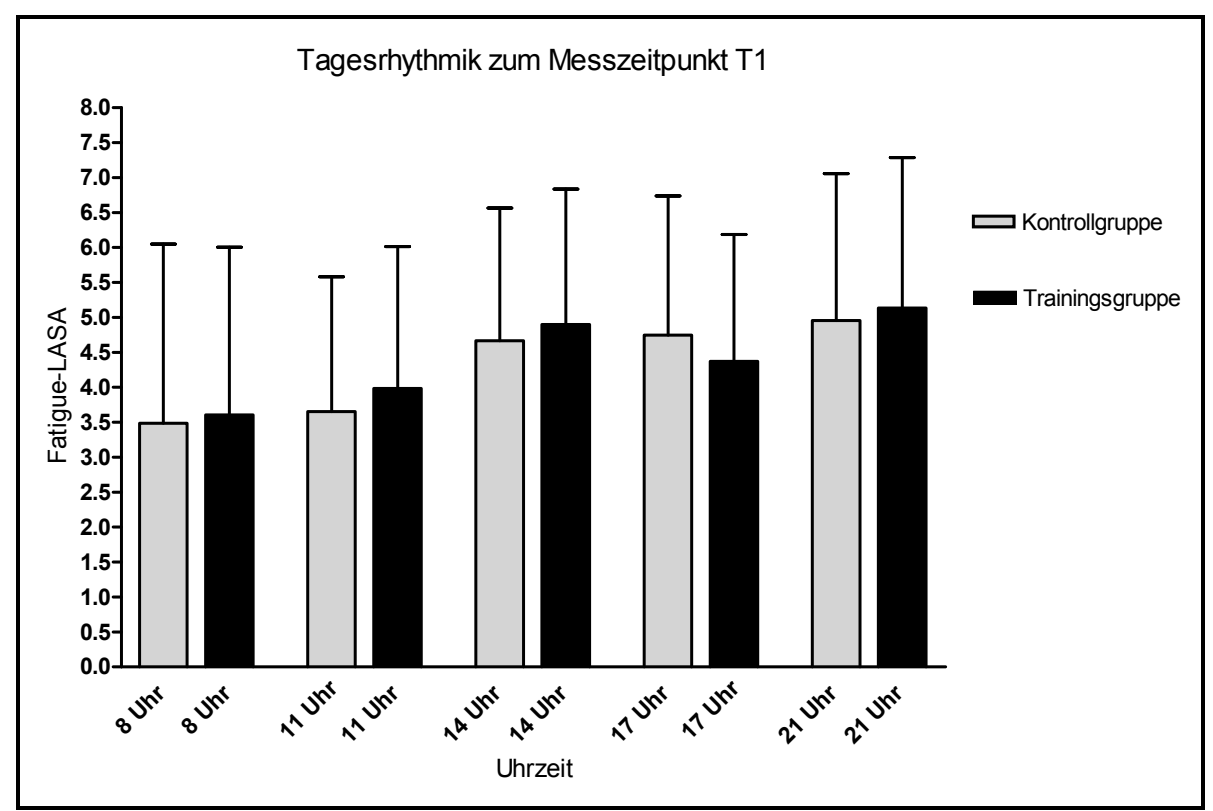

Abbildung 34: Vergleich Tagesrhythmik Fatigue Kontrollgruppe vs. Trainingsgruppe zu T1

\begin{tabular}{|l|r|r|r|}
\hline Uhrzeit & Kontrollgruppe & Trainingsgruppe & \multicolumn{1}{|c|}{$\begin{array}{c}\text { Signifikanz } \\
\text { K vs. Tr ( } \mathbf{p} \leq \mathbf{0 , 0 5})\end{array}$} \\
\hline $\mathbf{8} \mathbf{~ U h r}$ & $3,49 \pm 2,6$ & $3,61 \pm 2,4$ & 0,686 \\
\hline $\mathbf{1 1} \mathbf{~ U h r}$ & $3,65 \pm 1,9$ & $3,98 \pm 2,0$ & 0,159 \\
\hline $\mathbf{1 4} \mathbf{~ W r}$ & $4,67 \pm 1,9$ & $4,90 \pm 1,9$ & 0,311 \\
\hline $\mathbf{1 7} \mathbf{~ U h r}$ & $4,75 \pm 2,0$ & $4,37 \pm 1,8$ & 0,104 \\
\hline $\mathbf{2 1} \mathbf{~ U h r}$ & $4,96 \pm 2,1$ & $5,14 \pm 2,2$ & 0,481 \\
\hline
\end{tabular}

Tabelle 11: Vergleich Tagesrhythmik Fatigue Kontrollgruppe vs. Trainingsgruppe zu T1 


\subsubsection{Vergleich Kontroll- und Trainingsgruppe zum Messzeitpunkt T2}

Abbildung 35 zeigt den Vergleich der Fatiguewerte des Fatiguerhythmikbogens beider Gruppen zum Messzeitpunkt T2. Hierbei wurde deutlich, dass die Trainingsgruppe, mit Ausnahme der 8 Uhr Messung, zu allen Zeiten niedrigere Fatiguescores aufwies als die Kontrollgruppe. Um 14 Uhr und um 17 Uhr waren die Unterschiede zwischen Kontroll- und Trainingsgruppe signifikant. Die Signifikanzwerte aller Tageszeitpunkte können Tabelle 12 entnommen werden.

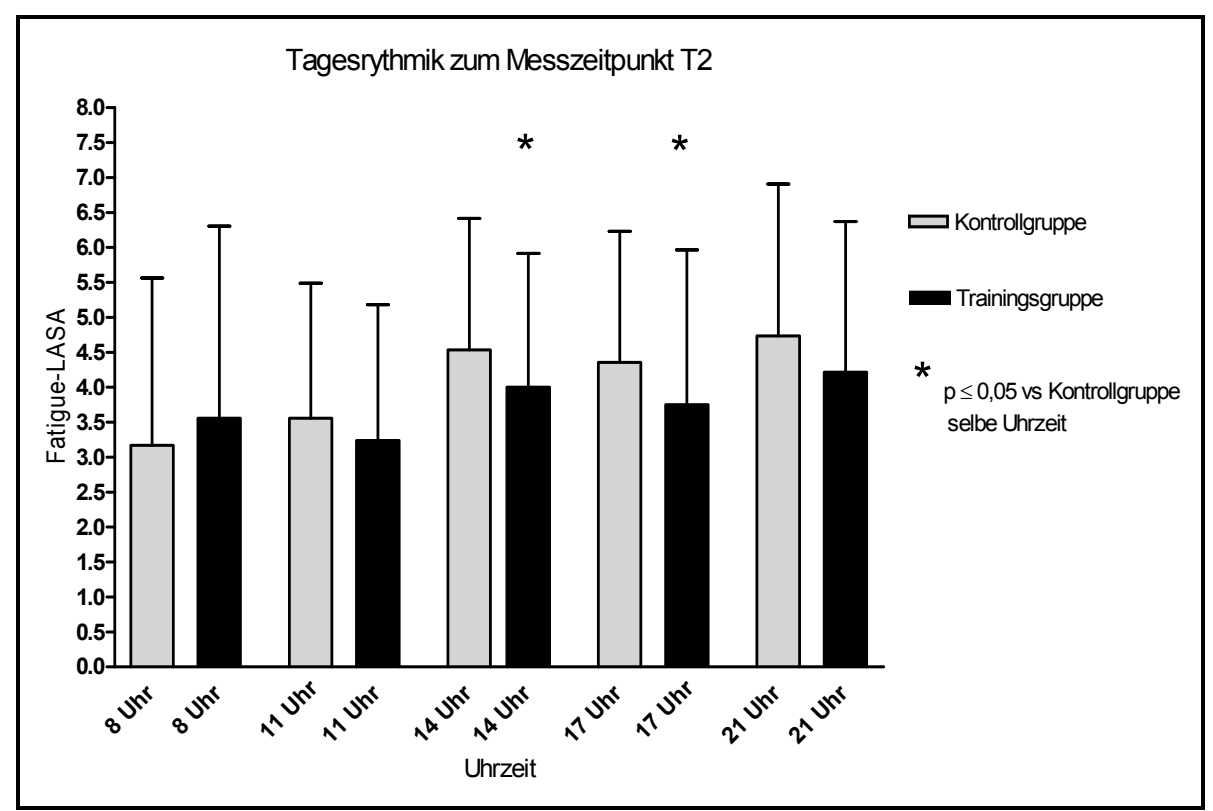

Abbildung 35: Vergleich Tagesrhythmik Fatigue Kontrollgruppe vs. Trainingsgruppe zu T2

\begin{tabular}{|l|r|r|r|}
\hline Uhrzeit & Kontrollgruppe & Trainingsgruppe & $\begin{array}{c}\text { Signifikanz } \\
\text { K vs. Tr ( } \mathbf{p} \leq \mathbf{0 , 0 5})\end{array}$ \\
\hline $\mathbf{8} \mathbf{~ U h r}$ & $3,17 \pm 2,4$ & $3,56 \pm 2,7$ & 0,276 \\
\hline $\mathbf{1 1} \mathbf{~ U h r}$ & $3,56 \pm 1,9$ & $3,24 \pm 1,8$ & 0,240 \\
\hline $\mathbf{1 4} \mathbf{~ W h}$ & $4,54 \pm 1,9$ & $4,00 \pm 1,9$ & $\mathbf{0 , 0 4 5}$ \\
\hline $\mathbf{1 7} \mathbf{~ U h r}$ & $4,37 \pm 1,9$ & $3,75 \pm 2,2$ & $\mathbf{0 , 0 3 3}$ \\
\hline $\mathbf{2 1} \mathbf{U h r}$ & $4,74 \pm 2,2$ & $4,20 \pm 2,2$ & 0,087 \\
\hline
\end{tabular}

Tabelle 12: Vergleich Tagesrhythmik Fatigue Kontrollgruppe vs. Trainingsgruppe zu T2 


\subsubsection{Vergleich Kontroll- und Trainingsgruppe zum Messzeitpunkt T3}

Zum Messzeitpunkt T3 war ein Unterschied des Fatiguscore auf dem Fatiguerhythmikbogen erkennbar. Der Fatiguescore der Trainingsgruppe war mit Ausnahme der 8 Uhr Messung zu allen Tageszeiten signifikant niedriger als der Score der Kontrollgruppe. Abbildung 36 zeigt das graphisch. In Tabelle 13 sind die Mittelwerte \pm Standardabweichungen inklusive der Signifikanzwerte des Gruppenvergleichs zwischen Kontroll- und Trainingsgruppe zusammengefasst.

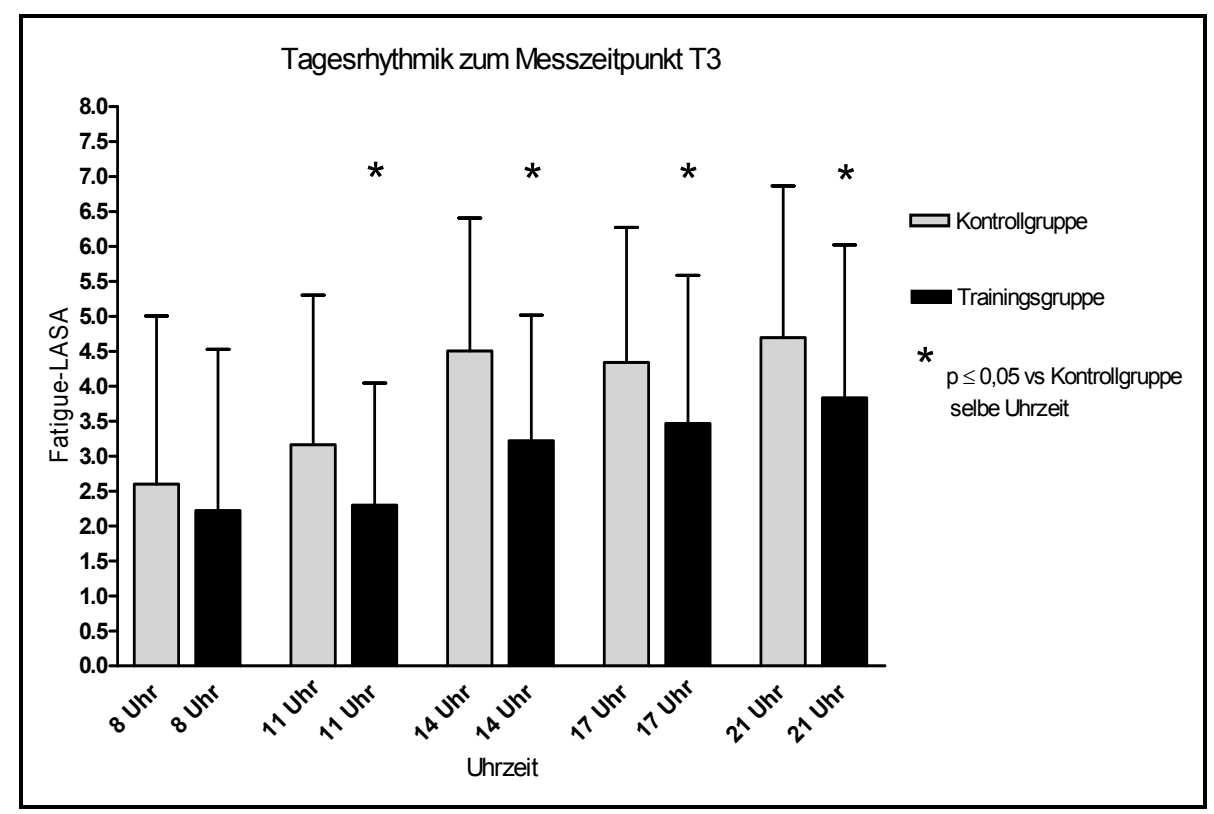

Abbildung 36: Vergleich Tagesrhythmik Fatigue Kontrollgruppe vs. Trainingsgruppe zu T3

\begin{tabular}{|l|r|r|r|}
\hline Uhrzeit & Kontrollgruppe & Trainingsgruppe & $\begin{array}{c}\text { Signifikanz } \\
\text { K vs. Tr ( } \mathbf{p} \leq \mathbf{0 , 0 5})\end{array}$ \\
\hline $\mathbf{8} \mathbf{~ U h r}$ & $2,60 \pm 2,4$ & $2,22 \pm 2,3$ & 0,2668 \\
\hline $\mathbf{1 1} \mathbf{~ U h r}$ & $3,16 \pm 2,1$ & $2,30 \pm 1,7$ & $\mathbf{0 , 0 0 2 6}$ \\
\hline $\mathbf{1 4} \mathbf{~ W r}$ & $4,51 \pm 1,9$ & $3,22 \pm 1,8$ & $<\mathbf{0 , 0 0 0 1}$ \\
\hline $\mathbf{1 7} \mathbf{~ U h r}$ & $4,34 \pm 1,9$ & $3,47 \pm 2,1$ & $\mathbf{0 , 0 0 2 9}$ \\
\hline $\mathbf{2 1} \mathbf{U h r}$ & $4,70 \pm 2,1$ & $3,83 \pm 2,2$ & $\mathbf{0 , 0 0 6 5}$ \\
\hline
\end{tabular}

Tabelle 13: Vergleich Tagesrhythmik Fatigue Kontrollgruppe vs. Trainingsgruppe zu T3 


\subsubsection{Schlaf und Aktivitätslevel}

\section{Schlafqualität}

Abbildung 37 zeigt die Schlafqualität beider Gruppen zu allen drei Messzeitpunkten. In der Kontrollgruppe konnten keine signifikanten Unterschiede zwischen den Messzeitpunkten nachgewiesen werden. Zu T1 lag der Mittelwert der Schlafqualität bei $5,20 \pm 2,1$. Zu T2 lag dieser bei $5,25 \pm 2,0$ und $z u T 3$ bei $5,41 \pm 1,9$. Die Schlafqualität in der Kontrollgruppe änderte sich nur marginal.

In der Trainingsgruppe zeigte sich ein anderes Bild. Die Werte zu T2 und T3 waren beide signifikant gegenüber dem Ausgangswert zu T1. Zu T1 lag der Mittelwert bei

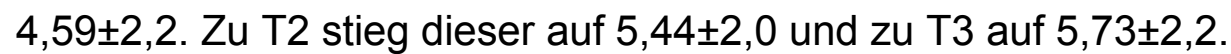
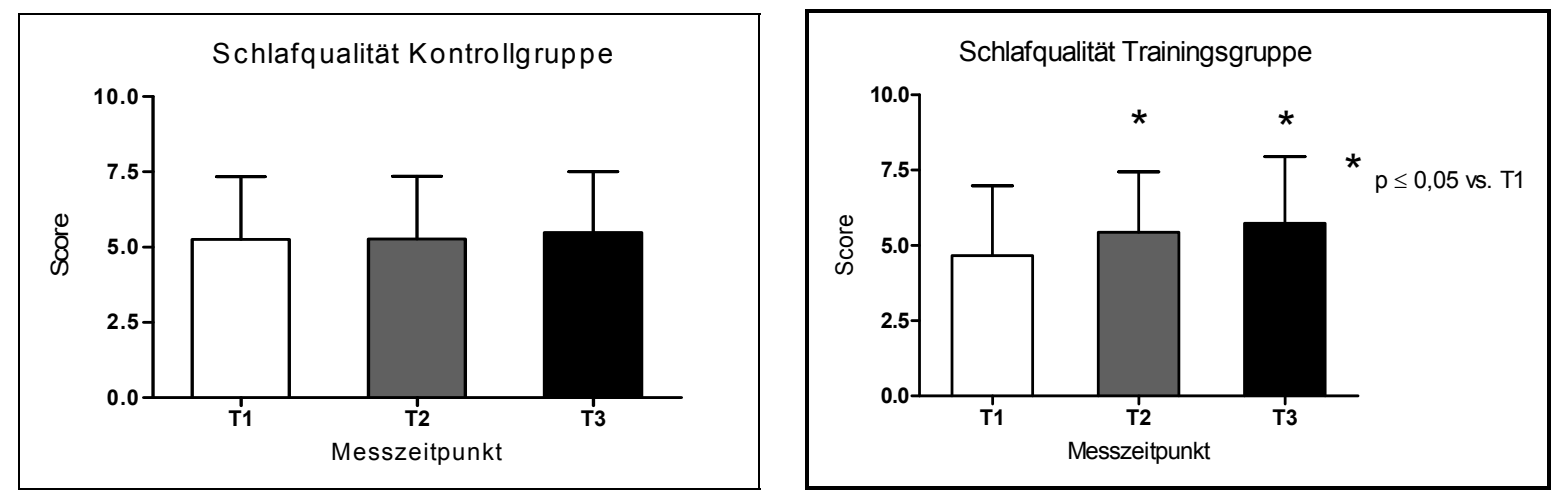

Abbildung 37: Schlafqualität Kontrollgruppe (links) und Trainingsgruppe (rechts)

\begin{tabular}{|l|r|r|r|r|r|r|}
\hline & \multicolumn{3}{|c|}{ Kontrollgruppe } & \multicolumn{3}{|c|}{ Trainingsgruppe } \\
\hline & T1 & T2 & T3 & T1 & T2 & T3 \\
\hline MEAN & 5,20 & 5,25 & 5,41 & 4,59 & 5,44 & 5,73 \\
\hline STDEV & 2,1 & 2,0 & 1,9 & 2,2 & 2,0 & 2,2 \\
\hline
\end{tabular}

Tabelle 14: Schlafqualität 
Aktivitätslevel

Der Aktivitätslevel wird in Abbildung 38 dargestellt. Es konnten nur in der Trainingsgruppe signifikante Veränderungen nachgewiesen werden. In der Kontrollgruppe waren die Unterschiede zwischen den Messzeitpunkten nur marginal.

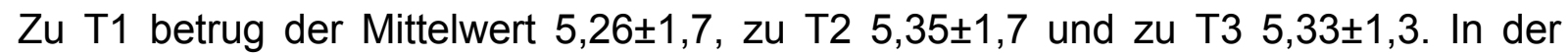

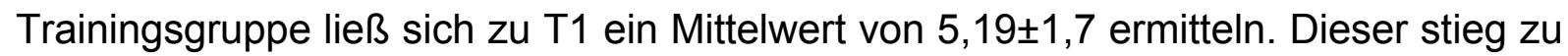
T2 auf $6.02 \pm 1,5$ an und betrug zu T3 5,74 $\pm 1,7$.
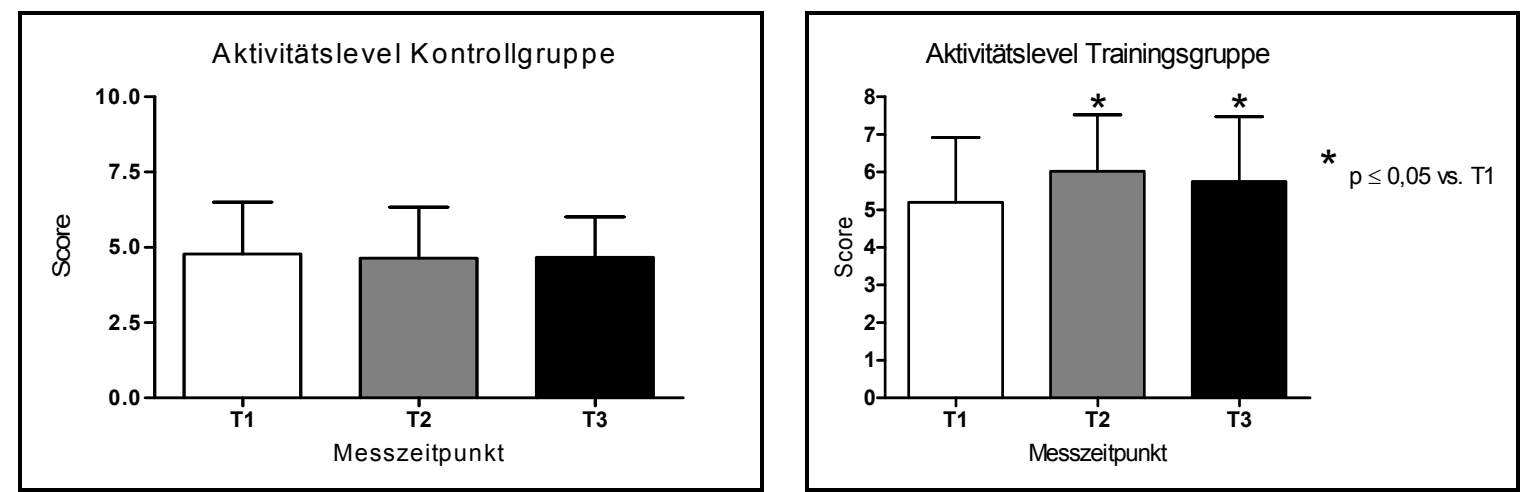

Abbildung 38: Aktivitätslevel Kontrollgruppe (links) und Trainingsgruppe (rechts)

\begin{tabular}{|l|r|r|r|r|r|r|}
\hline & \multicolumn{3}{|c|}{ Kontrollgruppe } & \multicolumn{3}{|c|}{ Trainingsgruppe } \\
\hline & T1 & T2 & T3 & T1 & T2 & T3 \\
\hline MEAN & 5,26 & 5,35 & 5,33 & 5,19 & 6,02 & 5,74 \\
\hline STDEV & 1,7 & 1,7 & 1,3 & 1,7 & 1,5 & 1,7 \\
\hline
\end{tabular}

Tabelle 15: Aktivitätslevel 


\subsubsection{Ergebnisse Angst und Depression (HADS)}

Angst

Der HADS beinhaltet eine Angst- und eine Depressionsskala. Diese Subskalen lassen sich getrennt voneinander auswerten. Die Skalen sind so ausgelegt, dass ein niedriger Score positiv für den Patienten ist.

Abbildung 39 zeigt Angstwerte der Kontroll- und der Trainingsgruppe zu allen drei Messzeitpunkten.

In der Kontrollgruppe zeigten sich keine signifikanten Veränderungen der

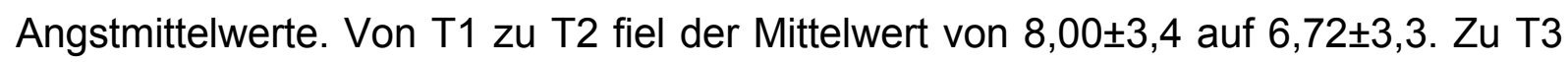
war ein Anstieg auf 6.91 $\pm 3,3$ zu erkennen.

Bei der Trainingsgruppe ließen sich signifikante Veränderungen von T2 und T3 gegenüber T1 nachweisen. Der T1 Wert lag bei 8,47 \pm 4,1 und sank zu T2 auf 4,96 \pm 3,1. Zu T3 sank der Angstwert weiter auf 4,78 $\pm 3,2$.
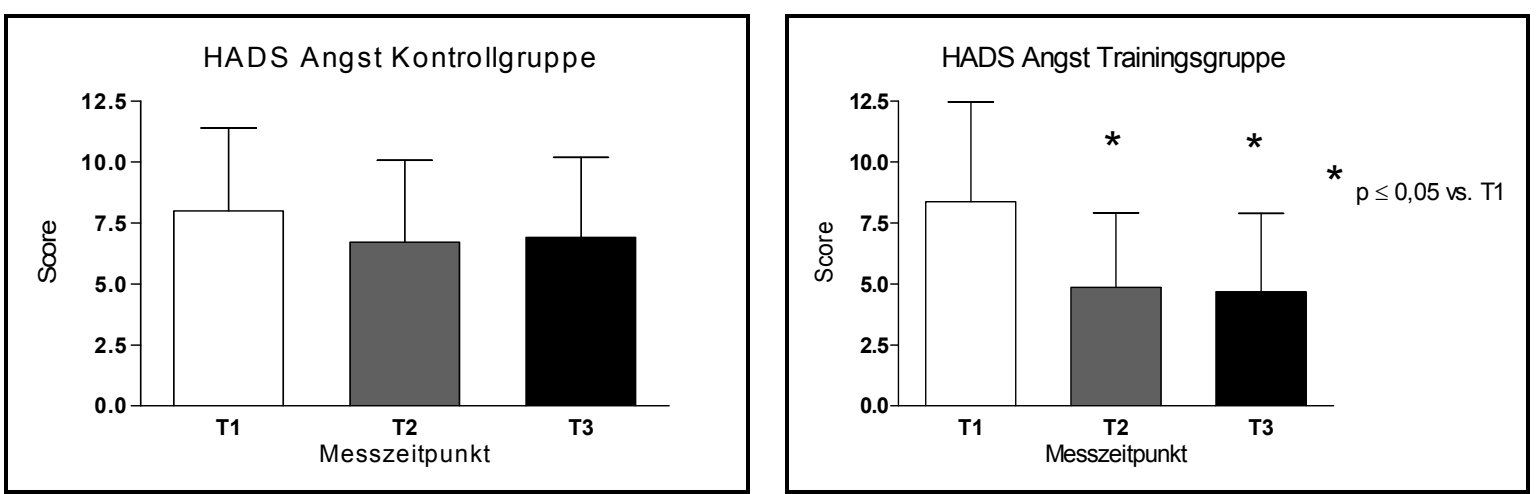

Abbildung 39: HADS Angstscore Kontrollgruppe (links) und Trainingsgruppe (rechts)

\begin{tabular}{|l|r|r|r|r|r|r|}
\hline & \multicolumn{3}{|c|}{ Kontrollgruppe } & \multicolumn{3}{|c|}{ Trainingsgruppe } \\
\hline & T1 & T2 & T3 & T1 & T2 & T3 \\
\hline MEAN & 8,00 & 6,72 & 6,91 & 8,47 & 4,96 & 4,78 \\
\hline STDEV & 3,4 & 3,3 & 3,3 & 4,1 & 3,1 & 3,2 \\
\hline
\end{tabular}

Tabelle 16: HADS Angstscore 
Depression

Abbildung 40 zeigt die Depressionswerte des HADS beider Gruppen zu den verschiedenen Messzeitpunkten.

In der Kontrollgruppe ließen sich keine signifikanten Veränderungen zwischen den verschiedenen Messzeitpunkten nachweisen. Allerdings sank der Depressionsscore

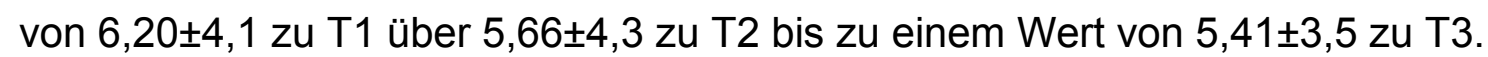

Die Werte der Trainingsgruppe veränderten sich signifikant gegenüber dem Ausgangswert von $6.05 \pm 3,6 \mathrm{zu}$ T1. Zu T2 lag ein Wert von 3,64 $\pm 2,8$ vor und zu T3 sank der Wert weiter auf 3,52 $\pm 2,6$.
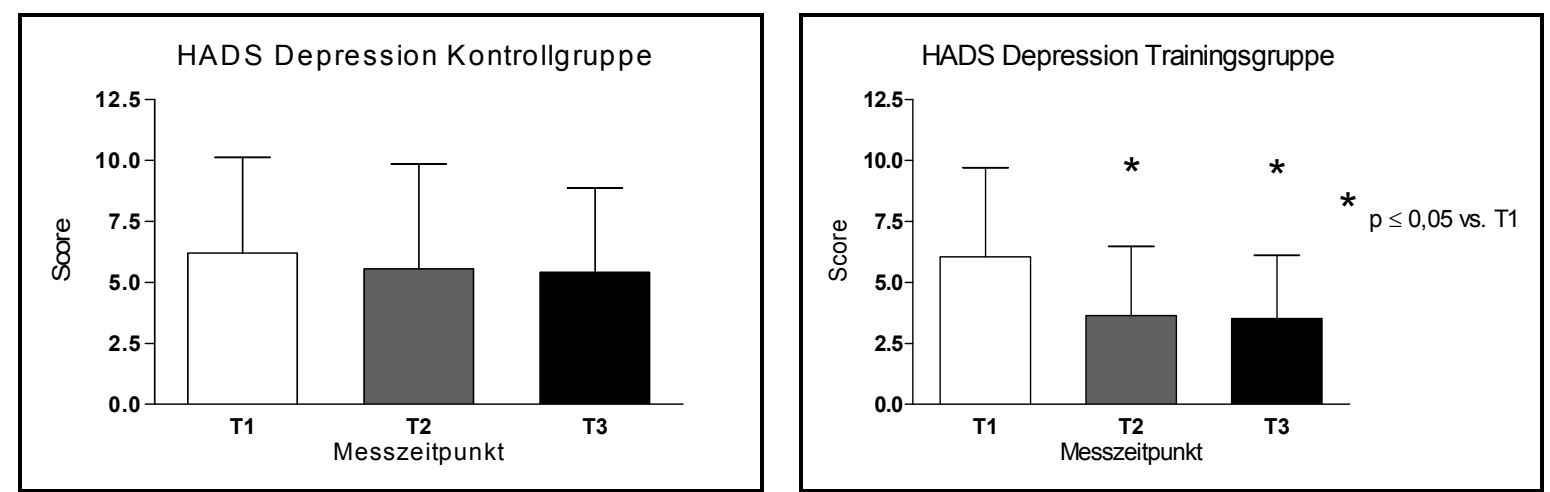

Abbildung 40: HADS Depressionsscore Kontrollgruppe (links) und Trainingsgruppe (rechts)

\begin{tabular}{|l|r|r|r|r|r|r|}
\hline & \multicolumn{3}{|c|}{ Kontrollgruppe } & \multicolumn{3}{c|}{ Trainingsgruppe } \\
\hline & T1 & T2 & T3 & T1 & T2 & T3 \\
\hline MEAN & 6,20 & 5,66 & 5,41 & 6,05 & 3,64 & 3,52 \\
\hline STDEV & 4,1 & 4,3 & 3,5 & 3,6 & 2,8 & 2,6 \\
\hline
\end{tabular}

Tabelle 17: HADS Depressionsscore 
6.2.10 Ergebnisse Multidimensional Fatigue Inventory (MFI)

In diesem Abschnitt werden die Ergebnisse der einzelnen MFI Subskalen dargestellt. Jede der folgenden Subskalen Allgemeine Fatigue, Physische Fatigue, Mentale Fatigue, Reduzierte Aktivität und Reduzierte Motivation wurde einzeln ausgewertet und veranschaulicht dargestellt. Beim MFI werden niedrige Scores als positiv betrachtet.

Allgemeine Fatigue

Aus Abbildung 41 wird ersichtlich, dass sowohl in der Kontroll- als auch in der Trainingsgruppe signifikante Veränderungen der Mittelwerte zu T2 und T3 gegenüber festzustellen waren. Für die Trainingsgruppe waren diese sogar hochsignifikant.

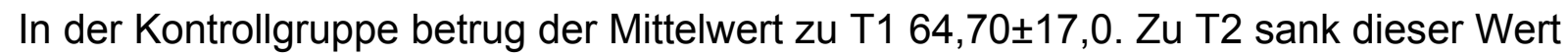
auf 56,25 $\pm 23,3$. T3 zeigte ein erneutes Sinken des Mittelwertes auf 51,79 $\pm 19,0$.

Die Trainingsgruppe zeigte ebenfalls einen Abfall des Score der Allgemeinen Fatigue. Ausgehend von einem Mittelwert von 69,79 $\pm 20,0$ fiel der Score zu T2 auf $47,77 \pm 26,5$. T3 zeigte einen Wert von 40,52 $\pm 23,3$.
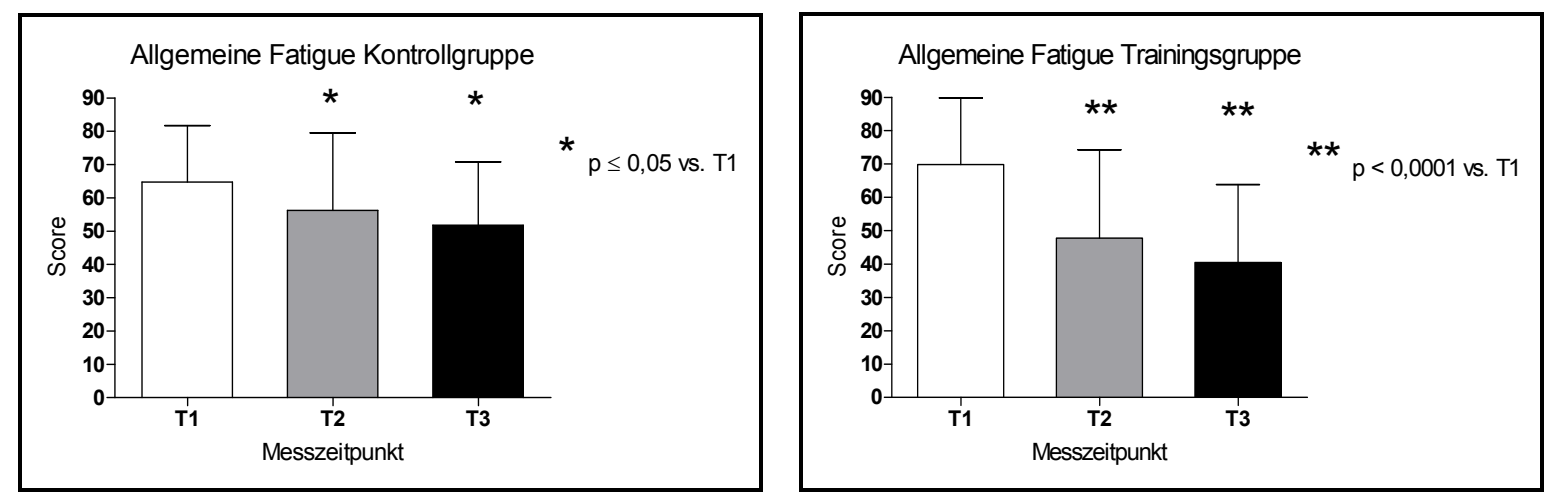

Abbildung 41: Allgemeine Fatigue Kontrollgruppe (links) und Trainingsgruppe (rechts)

\begin{tabular}{|l|r|r|r|r|r|r|}
\hline & \multicolumn{3}{|c|}{ Kontrollgruppe } & \multicolumn{3}{|c|}{ Trainingsgruppe } \\
\hline & T1 & T2 & T3 & T1 & T2 & T3 \\
\hline MEAN & 64,70 & 56,25 & 51,79 & 69,79 & 47,77 & 40,52 \\
\hline STDEV & 17,0 & 23,3 & 19,0 & 20,0 & 26,5 & 23,3 \\
\hline
\end{tabular}

Tabelle 18: Allgemeine Fatigue 
Physische Fatigue

Die Subskala der Physischen Fatigue zeigte ein ähnliches Bild. In Abbildung 42 ist erkennbar, dass es bei der Kontrollgruppe zu signifikanten Veränderungen von T2 und T3 gegenüber T1 kam. Erneut waren diese in der Trainingsgruppe hochsignifikant.

Die Mittelwerte der Kontrollgruppe fielen ausgehend von 58,33 $\pm 20,3$ zu T1 über 47,24 $\pm 24,6$ zu T2 bis hin zu 44,11 $\pm 19,6$ zu T3.

Die Ergebnisse der Trainingsgruppe fielen hochsignifikant aus. Zu T1 wurde ein Mittelwert von 64,29 $\pm 21,2$ ermittelt, der zu T2 bereits auf 37,95 $\pm 21,2$ gesunken war. Zu T3 sank der Wert erneut und wurde mit 35,96 $\pm 22,1$ gemessen.
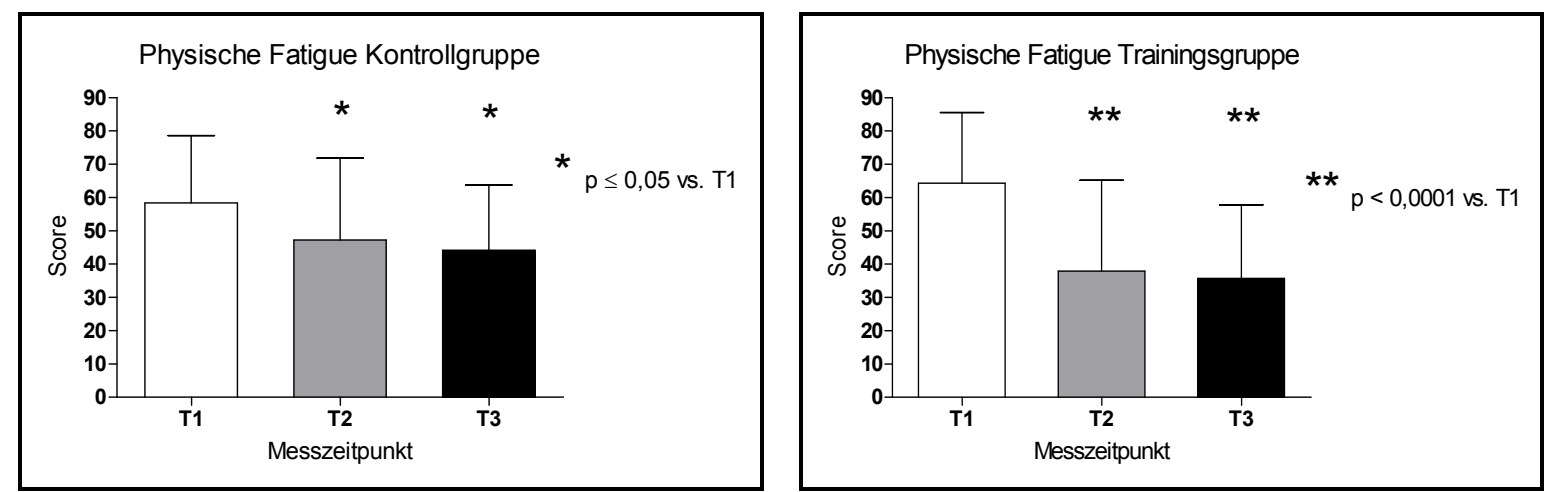

Abbildung 42: Physische Fatigue Kontrollgruppe (links) und Trainingsgruppe (rechts)

\begin{tabular}{|l|r|r|r|r|r|r|}
\hline & \multicolumn{3}{|c|}{ Kontrollgruppe } & \multicolumn{3}{c|}{ Trainingsgruppe } \\
\hline & T1 & T2 & T3 & T1 & T2 & T3 \\
\hline MEAN & 58,33 & 47,24 & 44,11 & 64,29 & 37,95 & 35,06 \\
\hline STDEV & 20,3 & 24,6 & 19,6 & 21,2 & 21,2 & 22,1 \\
\hline
\end{tabular}

Tabelle 19: Physische Fatigue 


\section{Mentale Fatigue}

Die Subskala Mentale Fatigue ist in Abbildung 43 graphisch dargestellt. In der Kontrollgruppe zeigten sich in der Subskala Mentaler Fatigue von T2 gegenüber T1 keine signifikanten Veränderungen. Erst zu T3 ließ sich Signifikanz gegenüber T1 nachweisen. Zu T1 betrug der Score für Mentale Fatigue 54,86 $\pm 26,5$. Dieser sank zu T2 auf 48,98 $\pm 24,7$ und zu T3 auf 38,93 $\pm 24,0$

In der Trainingsgruppe hingegen ließ sich bereits zwischen T1 und T2 eine hohe Signifikanz nachweisen. Die Mittelwerte zum Zeitpunkt T3 waren hochsignifikant gegenüber T1. Der Mittelwert zu T1 wurde mit 56,1 $\pm 22,3$ ermittelt und fiel zu T2

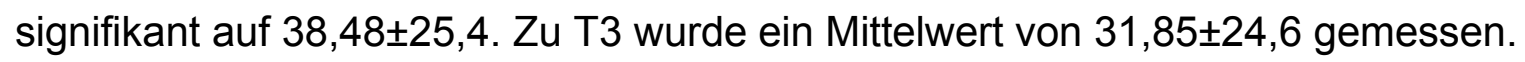
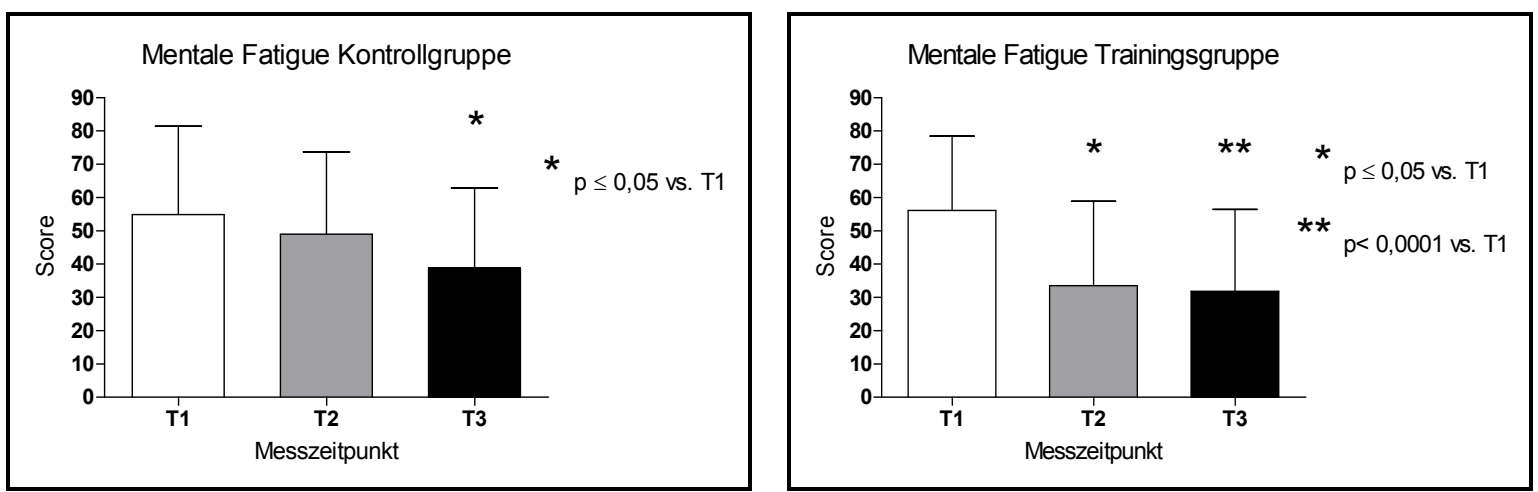

Abbildung 43: Mentale Fatigue Kontrollgruppe (links) und Trainingsgruppe (rechts)

\begin{tabular}{|l|r|r|r|r|r|r|}
\hline & \multicolumn{3}{|c|}{ Kontrollgruppe } & \multicolumn{3}{c|}{ Trainingsgruppe } \\
\hline & T1 & T2 & T3 & T1 & T2 & T3 \\
\hline MEAN & 54,86 & 48,98 & 39,39 & 56,1 & 38,48 & 31,85 \\
\hline STDEV & 26,5 & 24,7 & 24,0 & 22,3 & 25,4 & 24,6 \\
\hline
\end{tabular}

Tabelle 20: Mentale Fatigue 


\section{Reduzierte Aktivität}

In der Subskala Reduzierte Aktivität ließen sich in beiden Gruppen sowohl vom Messzeitpunkt T2 als auch von T3 signifikante Veränderungen der Mittelwerte gegenüber T1 nachweisen. Eine Darstellung findet sich in Abbildung 44.

Die Kontrollgruppe wies zu T1 einen Score von 52,78 $\pm 19,56$ auf. Dieser fiel zu T2 auf 42,59 $\pm 22,0$ und erreichte zu T3 mit 41,25 $\pm 20,0$ sein Minimum.

In der Trainingsgruppe fiel der Score ausgehend von $57,14 \pm 24,9$ zu T1 über $39,96 \pm 28,0$ zu T2 bis auf $34,88 \pm 20,9$ zu T3. Der Mittelwert zu T3 war hochsignifikant niedriger gegenüber T1.
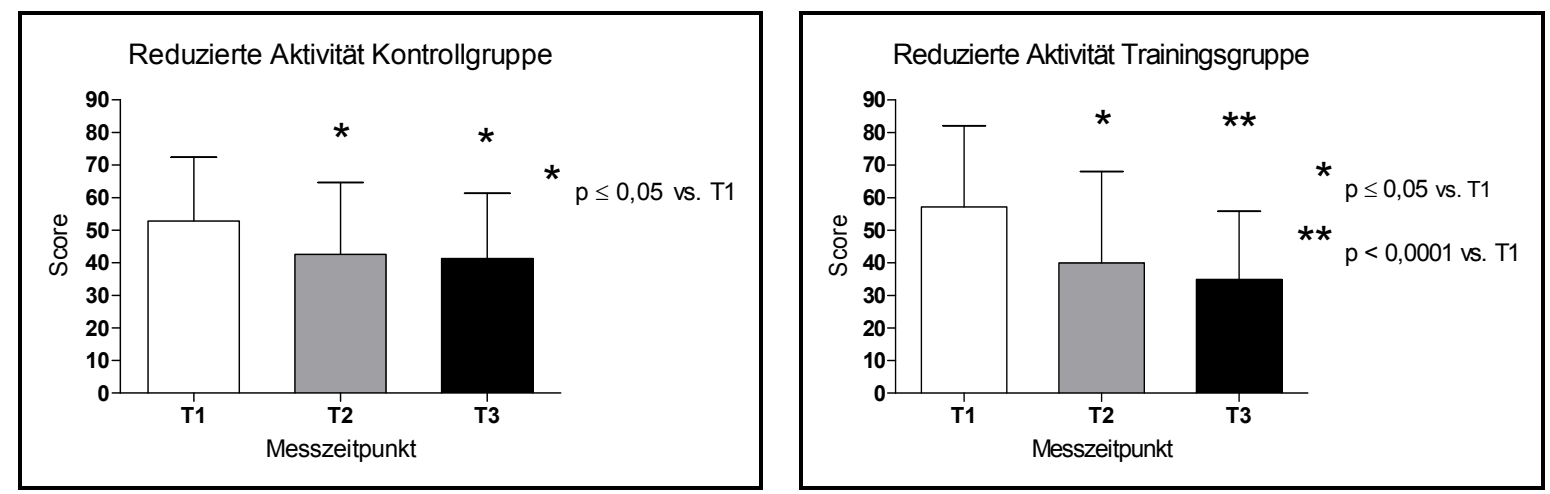

Abbildung 44: Reduzierte Aktivität Kontrollgruppe (links) und Trainingsgruppe (rechts)

\begin{tabular}{|l|r|r|r|r|r|r|}
\hline & \multicolumn{3}{|c|}{ Kontrollgruppe } & \multicolumn{3}{c|}{ Trainingsgruppe } \\
\hline & T1 & T2 & T3 & T1 & T2 & T3 \\
\hline MEAN & 52,78 & 42,59 & 41,25 & 57,14 & 39,96 & 34,88 \\
\hline STDEV & 19,56 & 22,0 & 20,0 & 24,9 & 28,0 & 20,9 \\
\hline
\end{tabular}

Tabelle 21: Reduzierte Aktivität 


\section{Reduzierte Motivation}

In der Subskala zu Reduzierter Motivation, Abbildung 45, ließen sich in der Kontrollgruppe keine signifikanten Veränderungen nachweisen. Der Mittelwert sank ausgehend von 35,65 $\pm 19,7 \mathrm{zu}$ T1 auf 33,72 $\pm 19,0 \mathrm{zu}$ T2. Zu T3 wurde ein erneut niedrigerer Wert von 28,75 $\pm 17,7$ ermittelt, der aber ebenfalls nicht signifikant gegenüber T1 war.

In der Trainingsgruppe hingegen ließen sich signifikante Veränderungen nachweisen. Ausgehend von $35,27 \pm 20,7$ zu T1 sank der Score zu T2 auf $25,67 \pm 17,5$ und zu T3 auf 23,39 $\pm 18,4$. Die Werte von T2 und T3 unterschieden sich signifikant von T1.
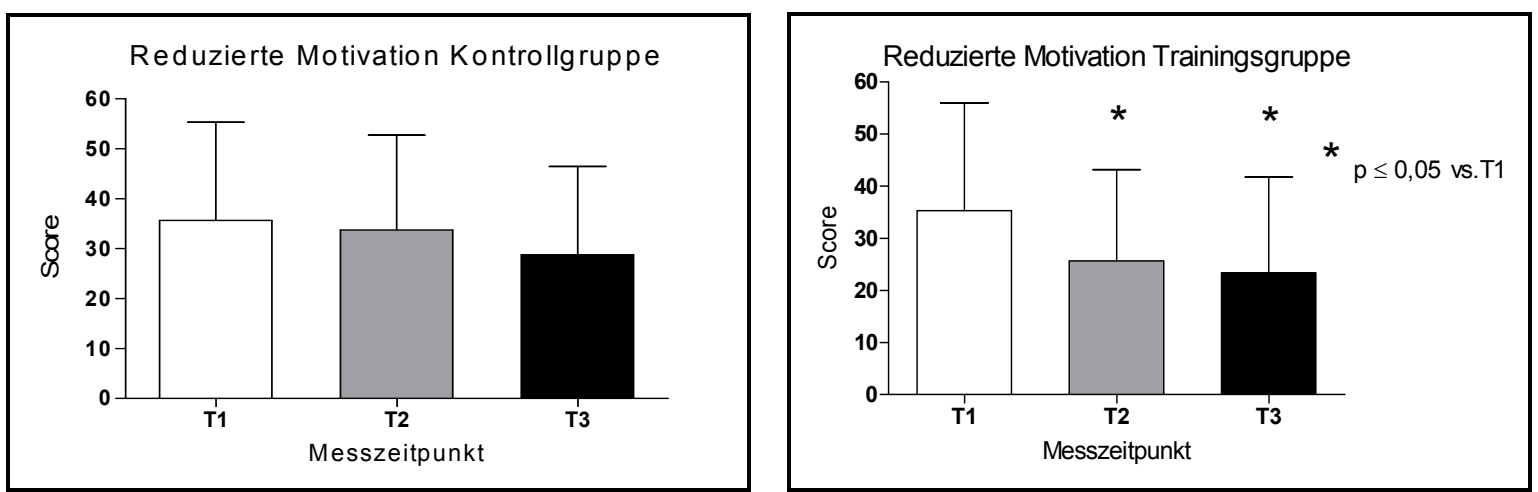

Abbildung 45: Reduzierte Motivation Kontrollgruppe (links) und Trainingsgruppe (rechts)

\begin{tabular}{|l|r|r|r|r|r|r|}
\hline & \multicolumn{3}{|c|}{ Kontrollgruppe } & \multicolumn{3}{c|}{ Trainingsgruppe } \\
\hline & T1 & T2 & T3 & T1 & T2 & T3 \\
\hline MEAN & 35,65 & 33,72 & 28,75 & 35,27 & 25,67 & 23,39 \\
\hline STDEV & 19,7 & 19,0 & 17,7 & 20,7 & 17,5 & 18,4 \\
\hline
\end{tabular}

Tabelle 22: Reduzierte Motivation 


\subsubsection{Ergebnisse European Organisation of Research and Treatment Cancer (EORTC QLQ-C30)}

Im folgenden Abschnitt werden die Ergebnisse des EORTC QLQ-C30 veranschaulicht dargestellt. Beim EORTC wird in Funktions- und Symptomskalen unterschieden. Hauptunterscheidungskriterium ist dabei, dass ein hoher Score bei Funktionsskalen positiv ist, während es sich bei Symptomskalen umgekehrt verhält.

Die Funktionsskalen sind Global Health, Physical Functioning, Cognitive Functioning, Emotional Functioning und Social Functioning. Fatigue, Schmerz und Schlaflosigkeit gehören zu den Symptomskalen.

\section{Global Health}

Die Subskala Global Health, Abbildung 46, zeigte sowohl in der Kontroll- als auch in der Trainingsgruppe signifikante Veränderungen.

In der Kontrollgruppe stieg der Score von $52,31 \pm 15,7$ zu T1 auf $62,79 \pm 19,9$ zu T2. Der Messzeitpunkt T3 zeigte einen fast identischen Wert von 62,75 $\pm 15,9$.

Ein ähnliches Bild zeigte sich in der Trainingsgruppe. Ausgangsmittelwert zu T1 war $51,59 \pm 17,7$. Dieser erhöhte sich zu T2 signifikant auf $68,10 \pm 18,5$ und fiel

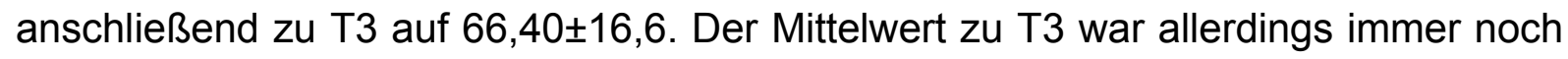
signifikant gegenüber T1.
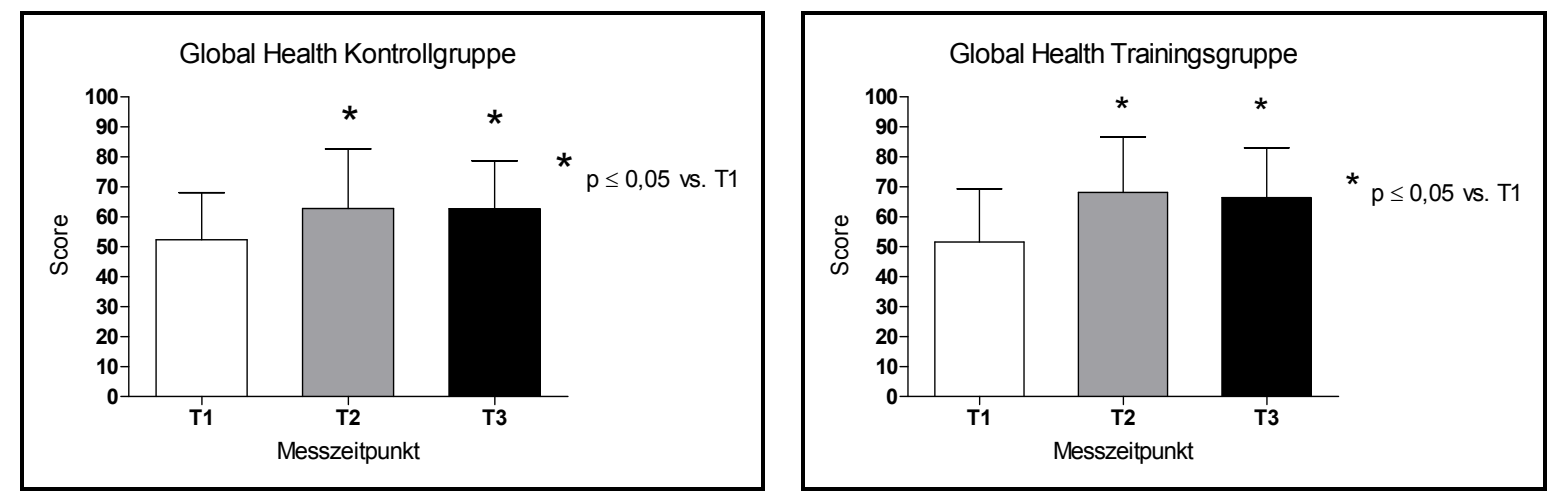

Abbildung 46: Global Health Kontrollgruppe (links) und Trainingsgruppe (rechts) 


\begin{tabular}{|l|r|r|r|r|r|r|}
\hline & \multicolumn{3}{|c|}{ Kontrollgruppe } & \multicolumn{3}{c|}{ Trainingsgruppe } \\
\hline & T1 & T2 & T3 & T1 & T2 & T3 \\
\hline MEAN & 52,31 & 62,79 & 62,75 & 51,59 & 68,10 & 66,40 \\
\hline STDEV & 15,7 & 19,9 & 15,9 & 17,7 & 18,5 & 16,6 \\
\hline
\end{tabular}

Tabelle 23: Global Health

\section{Physical Functioning}

Abbildung 47 zeigt die Subskala Physical Functioning. In dieser ließen sich in der Kontrollgruppe von T2 gegenüber T1 keine signifikanten Unterschiede nachweisen. Erst der Mittelwert zu T3 war signifikant höher als T1. Die Trainingsgruppe hingegen wies signifikante Unterschiede von T2 und T3 gegenüber T1 auf.

Der Mittelwert der Kontrollgruppe zu T1 betrug 64,94 $\pm 19,2$ stieg im Verlauf der Untersuchung auf $71,78 \pm 19,6$ zu T2 und auf 73,14 $\pm 14,7$ zu T3.

Die Trainingsgruppe zeigte einen deutlicheren Anstieg der Subskala Physical Functioning. Der Mittelwert zu T1 betrug 68,89 $\pm 17,7$. Im Verlauf der Studie stieg dieser auf $79,77 \pm 19,3$ zu T2 und auf $83,66 \pm 12,8$ zu T3.
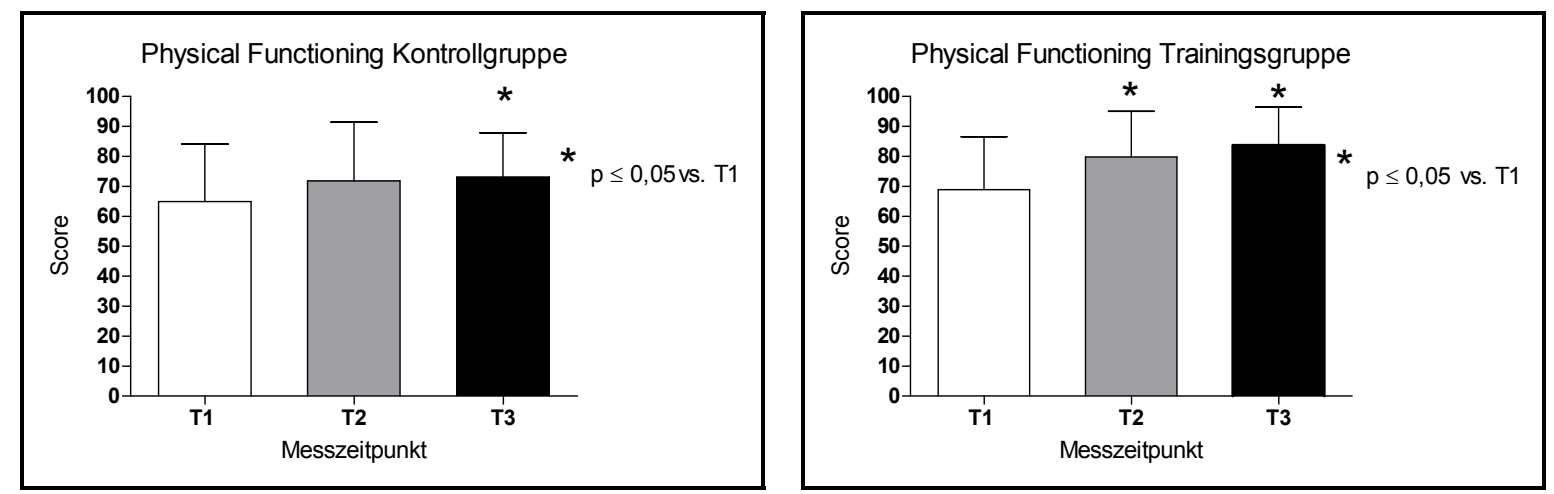

Abbildung 47: Physical Functioning Kontrollgruppe (links) und Trainingsgruppe (rechts)

\begin{tabular}{|l|r|r|r|r|r|r|}
\hline & \multicolumn{3}{|c|}{ Kontrollgruppe } & \multicolumn{3}{c|}{ Trainingsgruppe } \\
\hline & T1 & T2 & T3 & T1 & T2 & T3 \\
\hline MEAN & 64,94 & 71,78 & 73,14 & 68,89 & 79,77 & 83,66 \\
\hline STDEV & 19,2 & 19,6 & 14,7 & 17,7 & 19,3 & 12,8 \\
\hline
\end{tabular}

Tabelle 24: Physical Functioning 


\section{Emotional Functioning}

Der Mittelwert der Subskala Emotional Functioning stieg in beiden Gruppen von T1 zu T2 signifikant, in der Trainingsgruppe sogar hochsignifikant an. Von T2 zu T3 sank dieser allerdings wieder ab. Trotzdem blieben die Veränderungen signifikant gegenüber T1. Abbildung 48 zeigt dies grafisch.

In der Kontrollgruppe stiegen die Werte ausgehend von 50,77 $\pm 24,0$ zu T1 signifikant auf 63,95 $\pm 26,9$ zu T2. Zu T3 ließ sich ein Mittelwert von 61,27 $\pm 20,9$ ermitteln.

Ein ähnliches Bild zeigte sich in der Trainingsgruppe in der zu T1 ein Mittelwert von

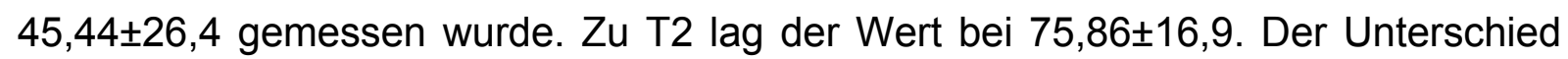
von T2 gegenüber T1 war hochsignifikant. Der Messzeitpunkt T3 zeigte analog zur Kontrollgruppe einen niedrigeren Mittelwert als zu T2. Dieser lag bei 65,86 $\pm 24,9$.
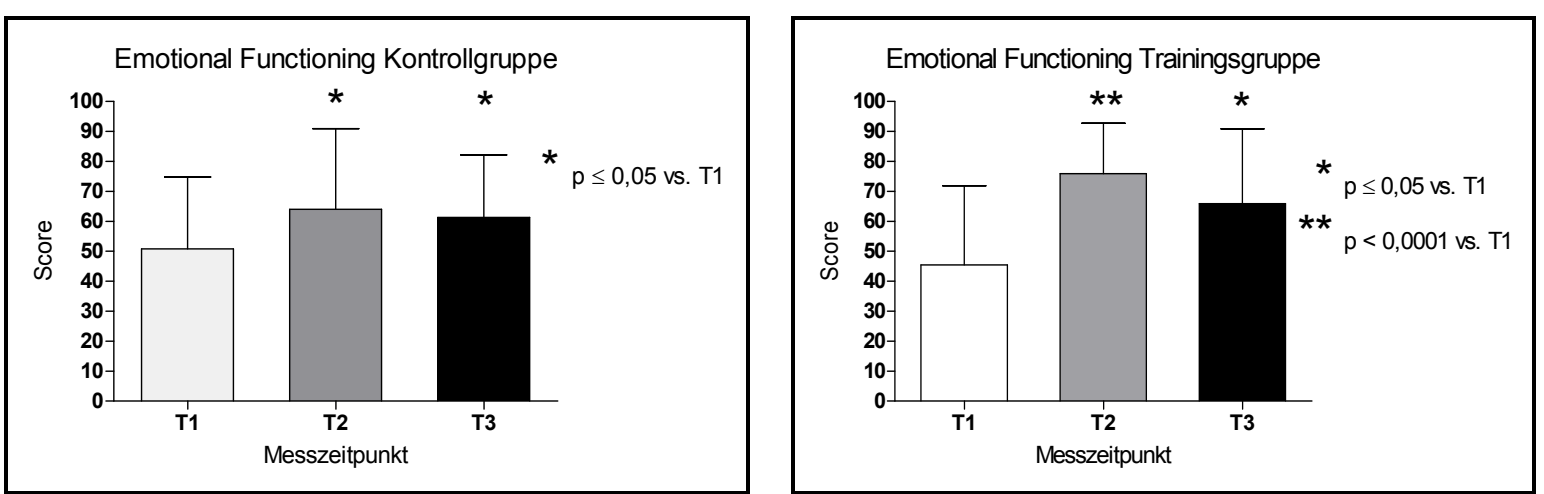

Abbildung 48: Emotional Functioning Kontrollgruppe (links) und Trainingsgruppe (rechts)

\begin{tabular}{|l|r|r|r|r|r|r|}
\hline & \multicolumn{3}{|c|}{ Kontrollgruppe } & \multicolumn{3}{|c|}{ Trainingsgruppe } \\
\hline & T1 & T2 & T3 & T1 & T2 & T3 \\
\hline MEAN & 50,77 & 63,95 & 61,27 & 45,44 & 75,86 & 65,86 \\
\hline STDEV & 24,0 & 26,9 & 20,9 & 26,4 & 16,9 & 24,9 \\
\hline
\end{tabular}

Tabelle 25: Emotional Functioning 
Cognitive Functioning

In der Subskala des Cognitive Functioning, Abbildung 49, ließen sich in beiden Gruppen nur signifikante Unterschiede zwischen T1 und T3 finden. Eine Steigerung des Mittelwertes von T1 zu T2 war aber immerhin in beiden Gruppen erkennbar.

Der Ausgangwert in der Kontrollgruppe wurde zu T1 mit 50,31 $\pm 31,6$ ermittelt. Zu T2 betrug dieser 58,91 $\pm 26,3$ und zu T3 67,65 $\pm 22,1$.

In der Trainingsgruppe stieg der Mittelwert von 52,38 $\pm 29,6$ zu T1 über $63,79 \pm 26,6$ zu T2 bis auf $72,58 \pm 27,1$ zu T3 an.
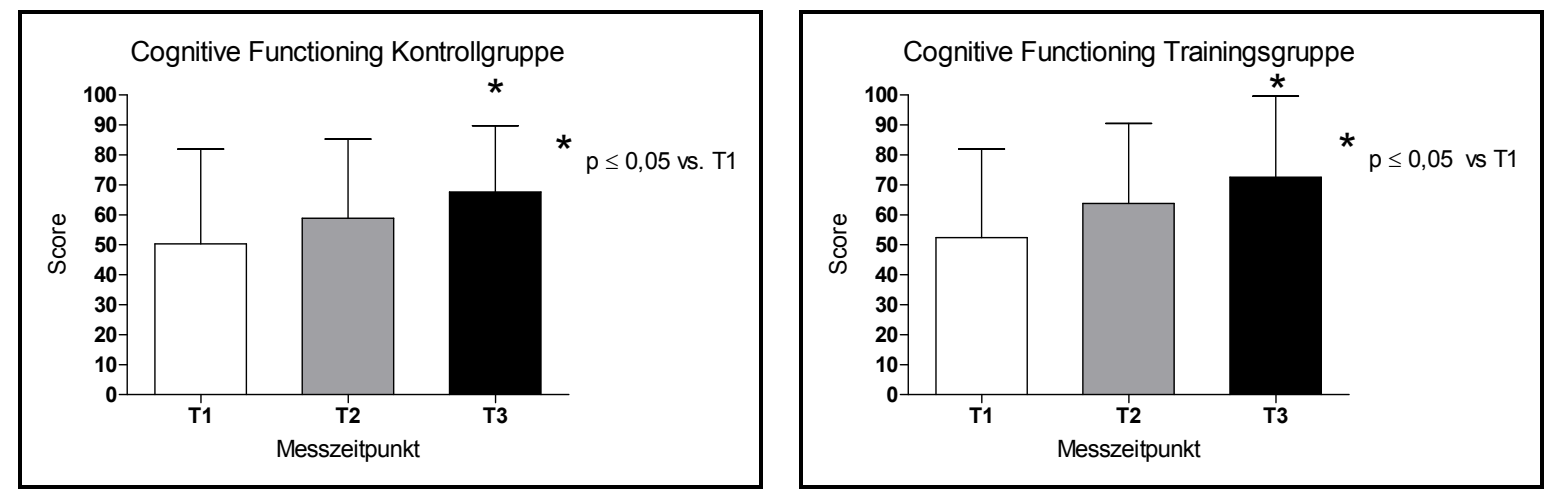

Abbildung 49: Cognitive Functioning Kontrollgruppe (links) und Trainingsgruppe (rechts)

\begin{tabular}{|l|r|r|r|r|r|r|}
\hline & \multicolumn{3}{|c|}{ Kontrollgruppe } & \multicolumn{3}{|c|}{ Trainingsgruppe } \\
\hline & T1 & T2 & T3 & T1 & T2 & T3 \\
\hline MEAN & 50,31 & 58,91 & 67,65 & 52,38 & 63,79 & 72,58 \\
\hline STDEV & 31,6 & 26,3 & 22,1 & 29,6 & 26,6 & 27,1 \\
\hline
\end{tabular}

Tabelle 26: Cognitive Functioning

\section{Social Functioning}

Die Subskala des Social Functioning zeigt in Abbildung 50 ein sehr differenziertes Ergebnis. Während in der Kontrollgruppe keine signifikanten Unterschiede der Mittelwerte zwischen den einzelnen Messzeitpunkten nachgewiesen werden konnten, war dies in der Trainingsgruppe möglich.

Der Mittelwert der Kontrollgruppe zu T1 betrug 58,95 $\pm 31,3$. Dieser erhöhte sich zu T2 auf $65,50 \pm 27,5$. Zu T3 erhöhte sich der Score weiter auf 70,10 $\pm 27,8$. 
In der Trainingsgruppe war der Mittelwert zum Anfangszeitpunkt T1 mit 51,59 $\pm 31,2$ niedriger als in der Kontrollgruppe. Zu T2 stieg der Wert auf 73,56 $\pm 26,9$, sank aber zu T3 auf 70,43 $\pm 28,1$. Die Unterschiede in der Trainingsgruppe von T2 und T3 waren gegenüber $\mathrm{T} 1$ signifikant.
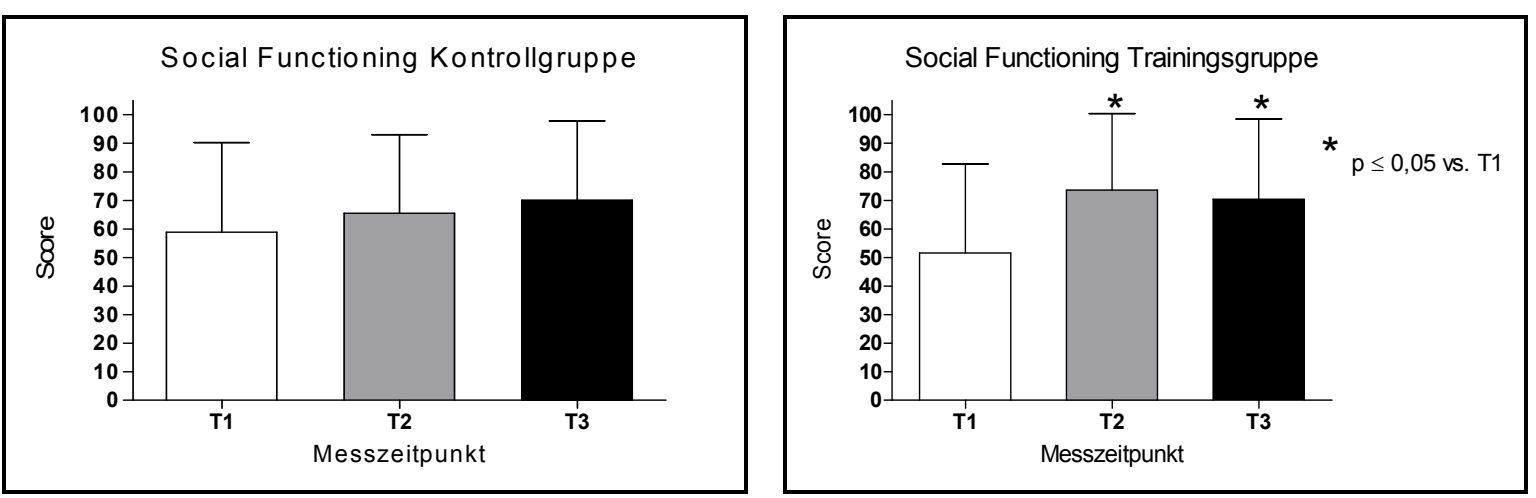

Abbildung 50: Social Functioning Kontrollgruppe (links) und Trainingsgruppe (rechts)

\begin{tabular}{|l|r|r|r|r|r|r|}
\hline & \multicolumn{3}{|c|}{ Kontrollgruppe } & \multicolumn{3}{c|}{ Trainingsgruppe } \\
\hline & T1 & T2 & T3 & T1 & T2 & T3 \\
\hline MEAN & 58,95 & 65,50 & 70,10 & 51,59 & 73,56 & 70,43 \\
\hline STDEV & 31,3 & 27,5 & 27,8 & 31,2 & 26,9 & 28,1 \\
\hline
\end{tabular}

Tabelle 27: Social Functioning 


\section{Fatigue}

Bei den folgenden Subskalen des EORTC QLQ-C30, einschließlich der Fatigueskala Abbildung 51, handelt es sich um Symptomskalen. Hierbei stehen wie schon erwähnt möglichst niedrige Scores für eine geringe Ausprägung des Symptoms.

In der Kontrollgruppe war erkennbar, dass ausgehend von einem Mittelwert von $63,75 \pm 19,9$ die Werte zu den Messzeitpunkten T2 und T3 signifikant kleiner wurden. Zu T2 wurde ein Mittelwert von 54,52 $\pm 24,1$ ermittelt, der zu T3 auf 47,71 $\pm 20,6$ sank. In der Trainingsgruppe sanken die Mittelwerte der Fatiguescore hochsignifikant. Die Werte zu den Messzeitpunkten T2 und T3 unterschieden sich hochsignifikant von einem Ausgangswert zu T1 von 67,20 $\pm 23,9$. Bereits zu T2 wurde ein Mittelwert von $44,06 \pm 21,7$ ermittelt, der zu T3 mit 37,99 $\pm 23,6$ weiter sank.
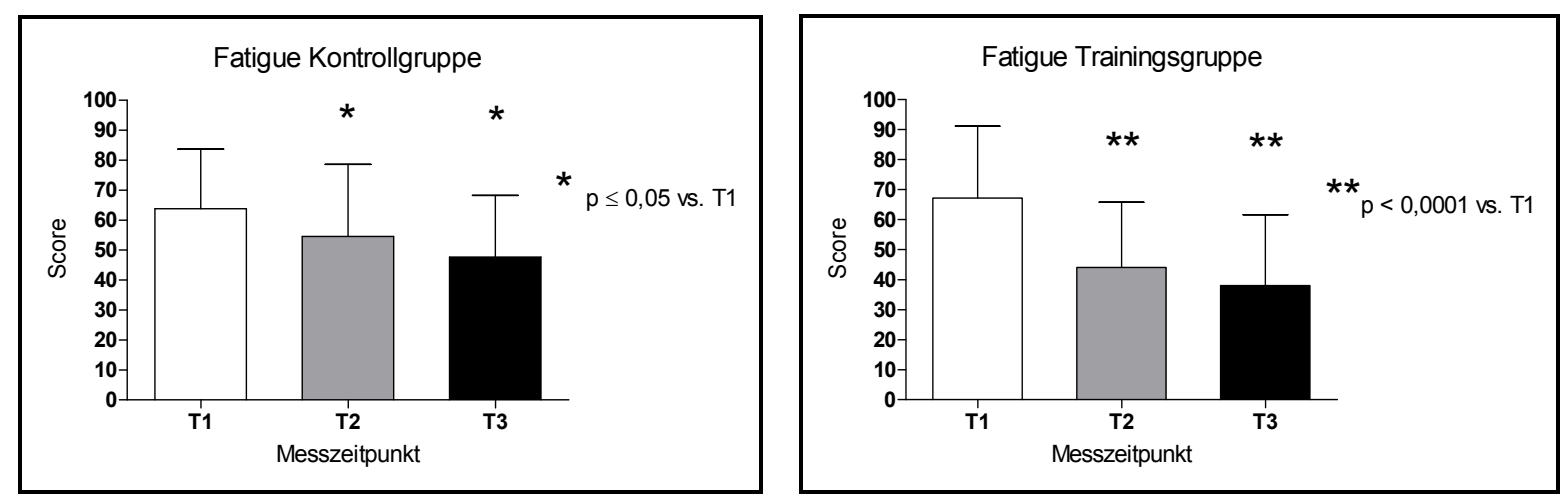

Abbildung 51: Fatigue Kontrollgruppe (links) und Trainingsgruppe (rechts)

\begin{tabular}{|l|r|r|r|r|r|r|}
\hline & \multicolumn{3}{|c|}{ Kontrollgruppe } & \multicolumn{3}{|c|}{ Trainingsgruppe } \\
\hline & T1 & T2 & T3 & T1 & T2 & T3 \\
\hline MEAN & 63,75 & 54,52 & 47,71 & 67,20 & 44,06 & 37,99 \\
\hline STDEV & 19,9 & 24,1 & 20,6 & 23,9 & 21,7 & 23,6 \\
\hline
\end{tabular}

Tabelle 28: Fatigue 


\section{Schmerz}

Die Symptomskala Schmerz brachte keine signifikanten Veränderungen hervor. In beiden Gruppen nahmen die Mittelwerte von T1 zu T2 ab und stiegen zum Zeitpunkt T3 wieder an.

In der Kontrollgruppe fiel der Mittelwert von 37,65 $\pm 31,1$ zu T1 auf $28,68 \pm 29,8$ zu T2 und stieg anschließend zu T3 wieder auf 36,23 $\pm 26,4$ an.

Eine ähnliche Entwicklung war in der Trainingsgruppe zu beobachten. Hier fiel der Mittelwert zunächst von $39,29 \pm 33,9$ zu T1 auf $26,44 \pm 31,9$ zu T2, um anschließend zu T3 wieder auf $29,72 \pm 29,5$ anzusteigen.
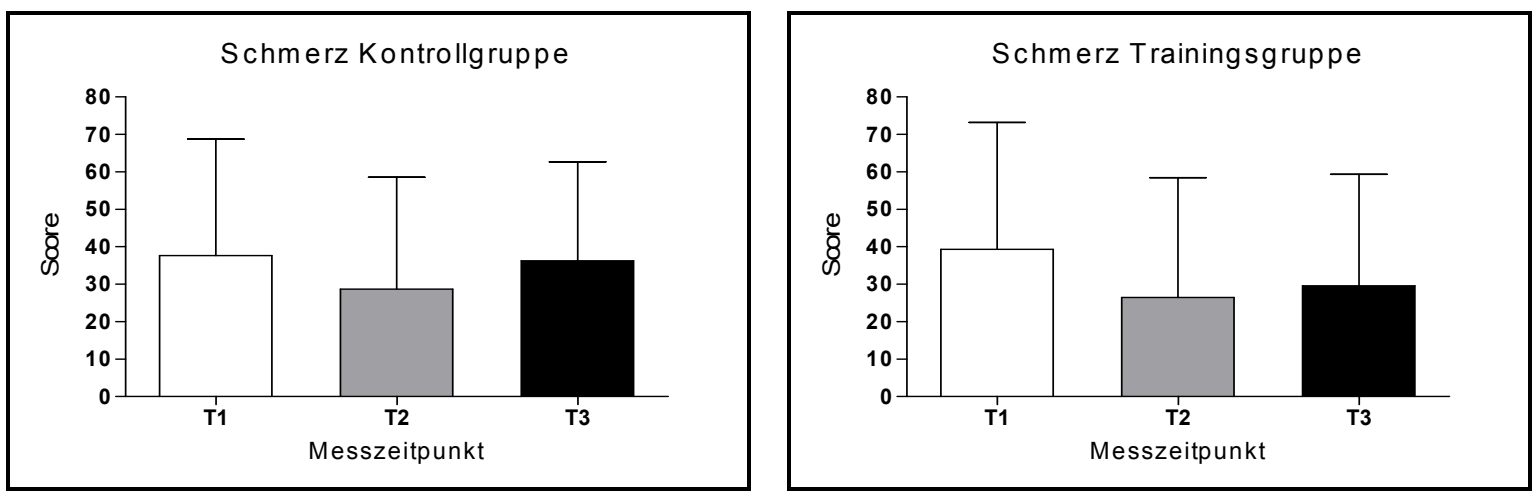

Abbildung 52: Schmerz Kontrollgruppe (links) und Trainingsgruppe (rechts)

\begin{tabular}{|l|r|r|r|r|r|r|}
\hline & \multicolumn{3}{|c|}{ Kontrollgruppe } & \multicolumn{3}{c|}{ Trainingsgruppe } \\
\hline & T1 & T2 & T3 & T1 & T2 & T3 \\
\hline MEAN & 37,65 & 28,68 & 36,23 & 39,29 & 26,44 & 29,72 \\
\hline STDEV & 31,1 & 29,8 & 26,4 & 33,9 & 31,9 & 29,5 \\
\hline
\end{tabular}

Tabelle 29: Schmerz

\section{Schlaflosigkeit}

Die Symptomskala Schlaflosigkeit zeigte ein sehr differenziertes Ergebnis. Während der Mittelwert in der Kontrollgruppe von T1 bis T3 kontinuierlich, aber nicht signifikant, anstieg, sank dieser in der Trainingsgruppe. In der Trainingsgruppe war eine signifikante Veränderung von T1 gegenüber T3 zu erkennen. 
Der Mittelwert der Schlaflosigkeit stieg in der Kontrollgruppe ausgehend von 43,83 $\pm 36,5$ zu T1 über 50,39 $\pm 35,9$ zu T2 bis zu einem Wert von $51,96 \pm 35,0$ zu T3. In der Trainingsgruppe fiel der Score von $61,9 \pm 35,7$ zu T1 über $51,72 \pm 36,3$ zu T2 bis auf $39,78 \pm 35,9$ zu T3.
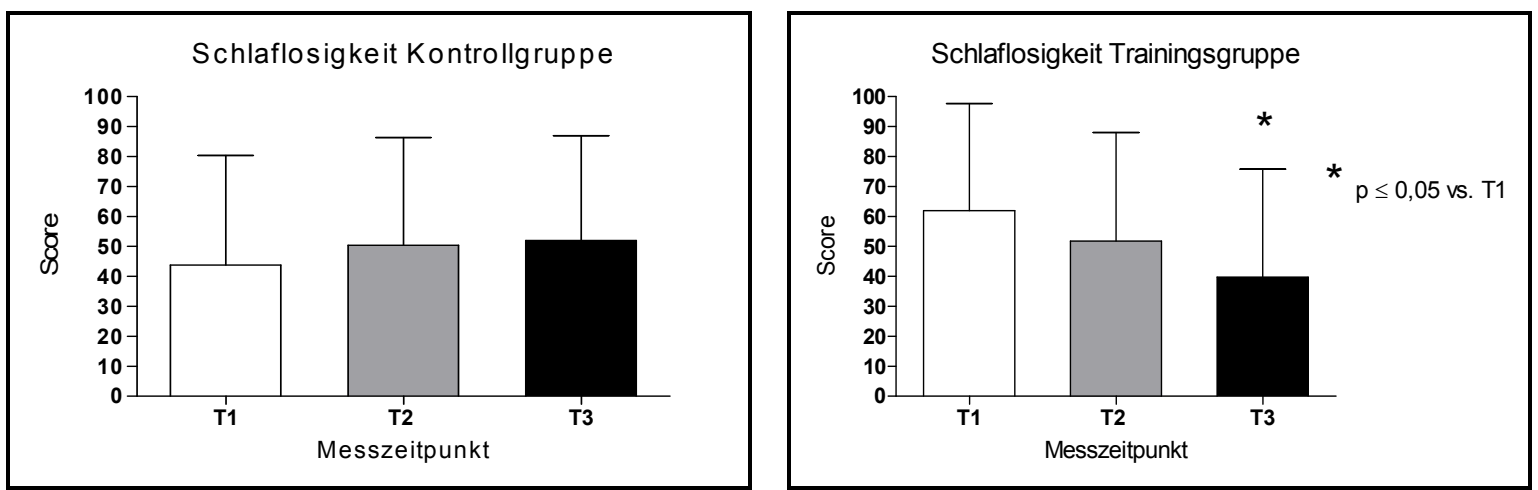

Abbildung 53: Schlaflosigkeit Kontrollgruppe (links) und Trainingsgruppe (rechts)

\begin{tabular}{|l|r|r|r|r|r|r|}
\hline & \multicolumn{3}{|c|}{ Kontrollgruppe } & \multicolumn{3}{|c|}{ Trainingsgruppe } \\
\hline & T1 & T2 & T3 & T1 & T2 & T3 \\
\hline MEAN & 43,83 & 50,39 & 51,96 & 61,90 & 51,72 & 39,78 \\
\hline STDEV & 36,5 & 35,9 & 35,0 & 35,7 & 36,6 & 35,9 \\
\hline
\end{tabular}

Tabelle 30: Schlaflosigkeit 
6.2.12 Ergebnisse Functional Assessment of Cancer Therapy-Fatigue Scale (FACIT-F)

Der FACIT-F hat einige Subskalen, die einzeln und unabhängig voneinander ausgewertet werden. Im folgenden Abschnitt werden die Ergebnisse der Subskalen Körperliches Wohlbefinden, Funktionsfähigkeit, Seelisches Wohlbefinden und Verhältnis zu Freunden und Bekannten dargestellt. Als positiv wird ein möglichst hoher Score definiert.

Körperliches Wohlbefinden

Die Subskala Körperliches Wohlbefinden, dargestellt in Abbildung 54, zeigte in beiden Gruppen hochsignifikante Unterschiede zwischen T2 und T3 gegenüber T1.

In der Kontrollgruppe stieg der Score ausgehend von $13,57 \pm 2,0$ auf $20,72 \pm 4,9$ zu T2 und stagnierte dann bei einem Wert von 20,89 $\pm 4,5$ zu T3.

Ähnlich verhielt es sich in der Trainingsgruppe. Mit einem Mittelwert von 18,45 $\pm 4,8$ zu T1 war der Ausgangsscore in der Trainingsgruppe höher als in der Kontrollgruppe. Zu T2 stieg dieser auf 23,25 $\pm 4,2$ um anschließend zu T3 auf 22,58 $\pm 4,0$ zu sinken.
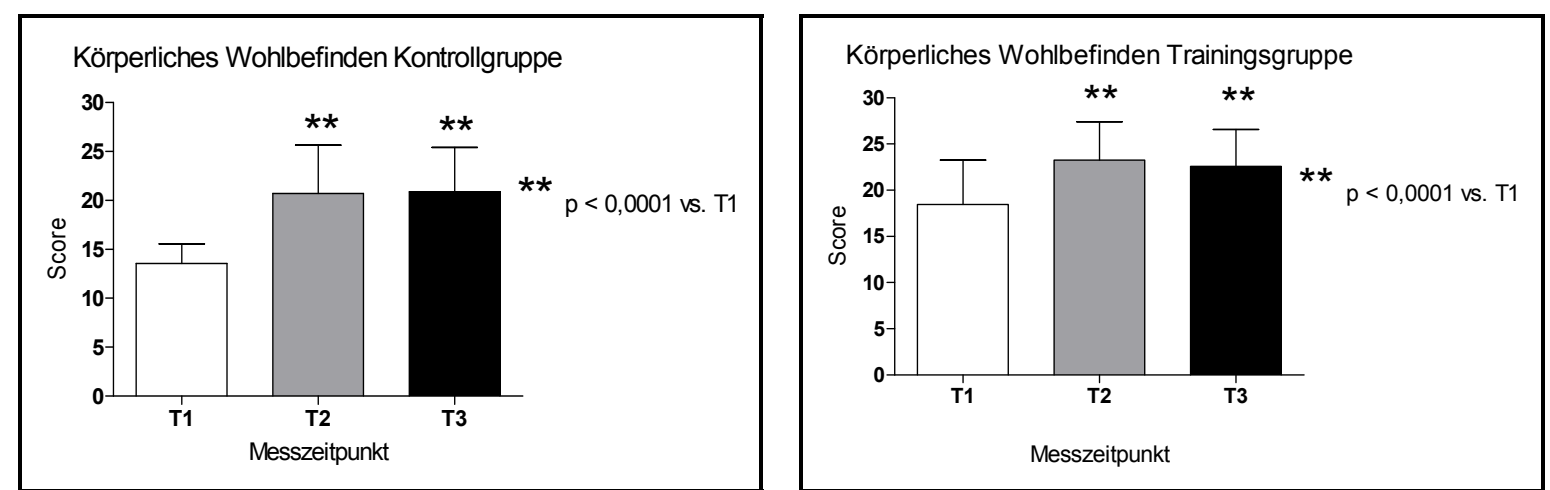

Abbildung 54: Körperliches Wohlbefinden Kontrollgruppe (links) und Trainingsgruppe (rechts)

\begin{tabular}{|l|r|r|r|r|r|r|}
\hline & \multicolumn{3}{|c|}{ Kontrollgruppe } & \multicolumn{3}{|c|}{ Trainingsgruppe } \\
\hline & T1 & T2 & T3 & T1 & T2 & T3 \\
\hline MEAN & 13,57 & 26,72 & 20,89 & 18,45 & 23,25 & 22,58 \\
\hline STDEV & 2,0 & 4,9 & 4,5 & 4,8 & 4,2 & 4,0 \\
\hline
\end{tabular}

Tabelle 31: Körperliches Wohlbefinden 


\section{Funktionsfähigkeit}

Bei der Subskala Funktionsfähigkeit konnten in der Kontrollgruppe keine signifikanten Veränderungen zwischen den drei Messzeitpunkten ermittelt werden. Abbildung 53 ist zu entnehmen, dass der Ausgangswert von 16,81 $\pm 5,1$ zu T1 sich im

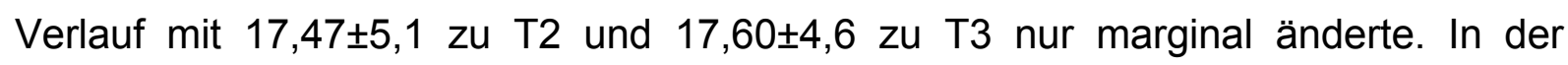
Trainingsgruppe hingegen war ein hochsignifikanter Anstieg der Mittelwerte von 16,12 $\pm 5,6$ zu T1 auf 22,29 $\pm 4,0$ zu T2 festzustellen. Zu T3 fiel der Wert auf 19,97 $\pm 4,8$, welcher aber immer noch signifikant gegenüber T1 war.
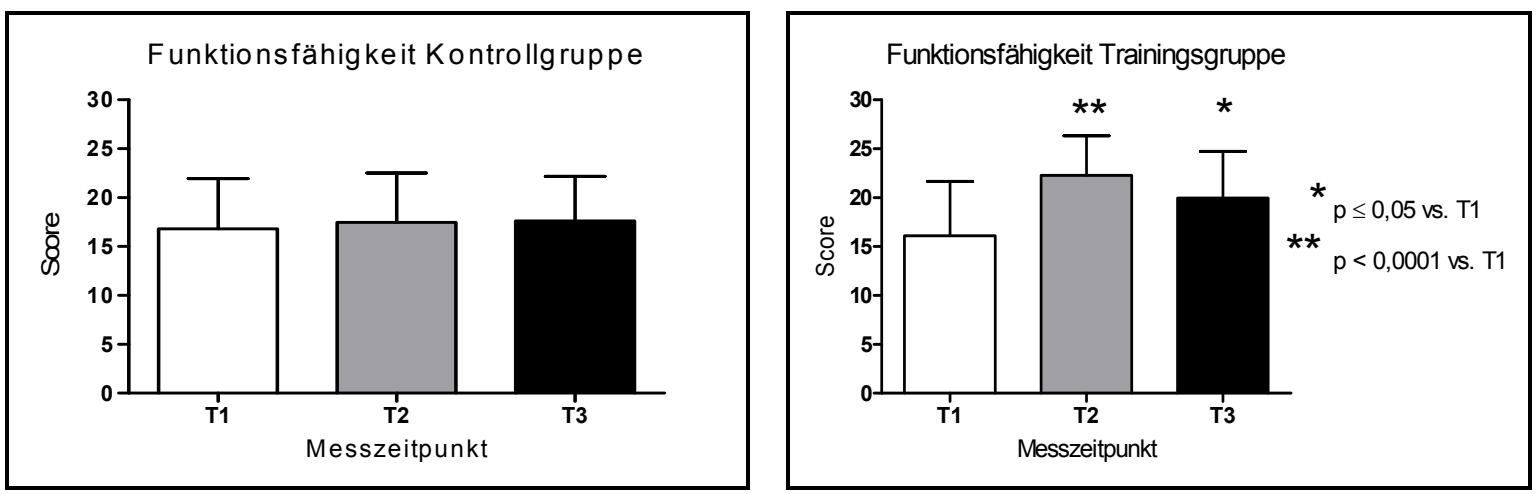

Abbildung 55: Funktionsfähigkeit Kontrollgruppe (links) und Trainingsgruppe (rechts)

\begin{tabular}{|l|r|r|r|r|r|r|}
\hline & \multicolumn{3}{|c|}{ Kontrollgruppe } & \multicolumn{3}{|c|}{ Trainingsgruppe } \\
\hline & T1 & T2 & T3 & T1 & T2 & T3 \\
\hline MEAN & 16,81 & 17,47 & 17,60 & 16,12 & 22,29 & 19,97 \\
\hline STDEV & 5,1 & 5,1 & 4,6 & 5,6 & 4,0 & 4,8 \\
\hline
\end{tabular}

Tabelle 32: Funktionsfähigkeit

Seelisches Wohlbefinden

Die Ergebnisse der Subskala Seelisches Wohlbefinden zeigten in der Kontrollgruppe keine signifikanten Unterschiede. Allerdings ließen sich erneut signifikante Unterschiede in der Trainingsgruppe ermitteln.

Ausgehend von 16,44 $\pm 4,1$ zu T1 veränderte sich der Score Seelisches Wohlbefinden mit 16,58 $\pm 4,9$ zu T2 und 17,26 $\pm 4,0$ zu T3 nur marginal. 
Dem hingegen stieg der Score in der Trainingsgruppe ausgehend von 17,56 $\pm 3,8 \mathrm{zu}$ T1 signifikant auf 20,00 $\pm 4,0$ zu T2 an, fiel aber dann zu T3 auf 19,35 $\pm 3,3$.
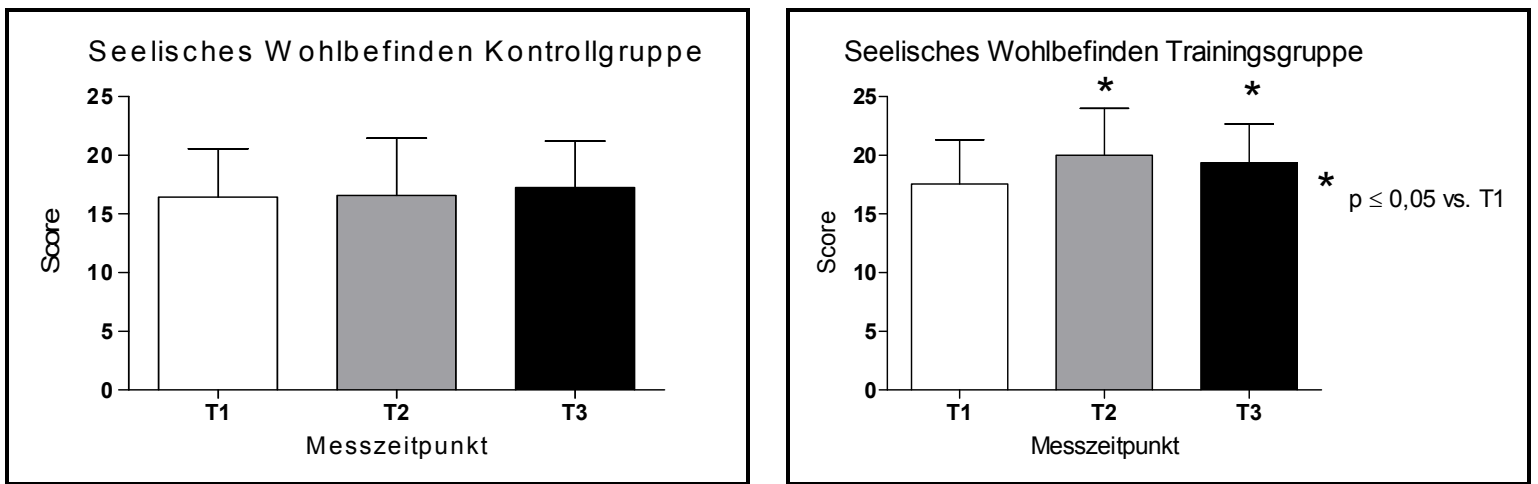

Abbildung 56: Seelisches Wohlbefinden Kontrollgruppe (links) und Trainingsgruppe (rechts)

\begin{tabular}{|l|r|r|r|r|r|r|}
\hline & \multicolumn{3}{|c|}{ Kontrollgruppe } & \multicolumn{3}{|c|}{ Trainingsgruppe } \\
\hline & T1 & T2 & T3 & T1 & T2 & T3 \\
\hline MEAN & 16,44 & 16,58 & 17,26 & 17,56 & 20,00 & 19,35 \\
\hline STDEV & 4,1 & 4,9 & 4,0 & 3,8 & 4,0 & 3,3 \\
\hline
\end{tabular}

Tabelle 33: Seelisches Wohlbefinden 
Verhältnis zu Freunden und Familie

Abbildung 57 zeigt die Subskala Verhältnis zu Freunden und Verwandten. Diese änderte sich in beiden Gruppen nicht signifikant. In der Kontrollgruppe war ein Abfall der Mittelwerte zu beobachten, während in der Trainingsgruppe ein Anstieg erkennbar war.

Ausgehend von $20,59 \pm 4,9$ zu T1 sank der Wert zu T2 auf 20,28 $\pm 4,0$ und zu T3 auf $18,57 \pm 5,3$.

In der Trainingsgruppe wurde zum Messzeitpunkt T1 ein Mittelwert von 20,98 $\pm 5,5$ ermittelt. Dieser stieg im Verlauf der Untersuchung auf 22,29 $\pm 4,0$ zu T2 und anschließend auf 22,94 $\pm 3,4$ zu T3.
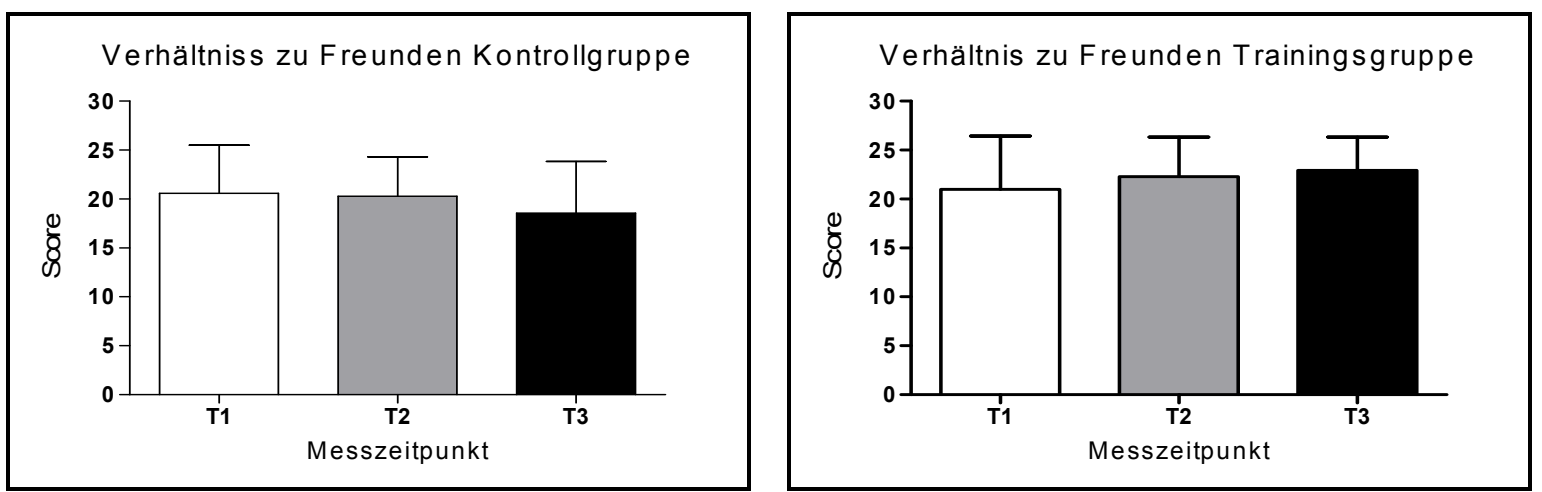

Abbildung 57: Verhältnis zu Freunden und Familie Kontrollgruppe (links) und Trainingsgruppe (rechts)

\begin{tabular}{|l|r|r|r|r|r|r|}
\hline & \multicolumn{3}{|c|}{ Kontrollgruppe } & \multicolumn{3}{|c|}{ Trainingsgruppe } \\
\hline & T1 & T2 & T3 & T1 & T2 & T3 \\
\hline MEAN & 20,59 & 20,28 & 18,57 & 20,98 & 22,29 & 22,94 \\
\hline STDEV & 4,9 & 4,0 & 5,3 & 5,5 & 4,0 & 3,4 \\
\hline
\end{tabular}

Tabelle 34: Verhältnis zu Freunden und Familie 


\section{Fatigue}

Das Fatiguemodul des FACIT-F ist in Abbildung 58 dargestellt. Zwischen T1 und T2 ließen sich in der Kontrollgruppe keine signifikanten Unterschiede nachweisen. Der

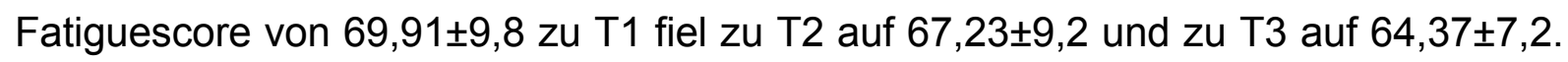
Der Mittelwert zu T3 ist gegenüber T1 signifikant.

In der Trainingsgruppe zeigt sich, dass T2 und T3 hochsignifikant gegenüber T1 sind. Der Mittelwert der Subskala Fatigue liegt zu T1 bei $73,93 \pm 12,1$ und fällt zu T2 auf $60,82 \pm 10,0$. Zu T3 liegt der Mittelwert der Fatigue bei 60,35 $\pm 9,4$.
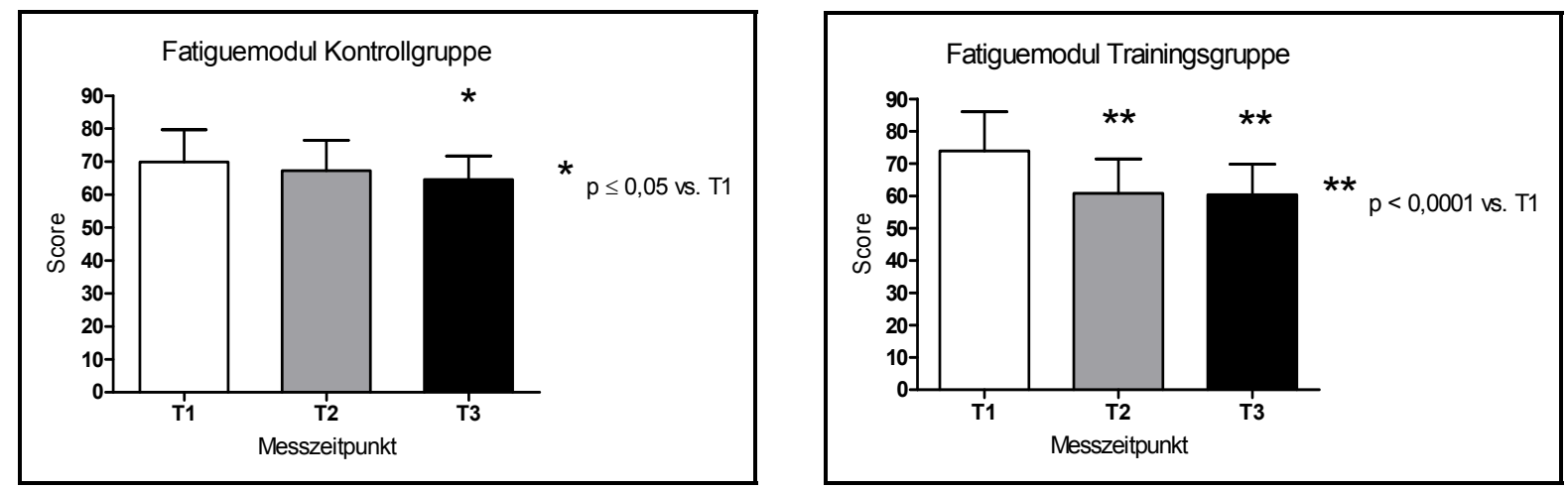

Abbildung 58: Fatiguemodul Kontrollgruppe (links) und Trainingsgruppe (rechts)

\begin{tabular}{|l|r|r|r|r|r|r|}
\hline & \multicolumn{3}{|c|}{ Kontrollgruppe } & \multicolumn{3}{|c|}{ Trainingsgruppe } \\
\hline & T1 & T2 & T3 & T1 & T2 & T3 \\
\hline MEAN & 69,91 & 67,23 & 64,37 & 73,93 & 60,82 & 60,35 \\
\hline STDEV & 9,8 & 9,2 & 7,2 & 12,1 & 10,0 & 9,4 \\
\hline
\end{tabular}

Tabelle 35: Fatiguemodul 


\subsection{Leistungsdiagnostik}

\subsubsection{Ergebnisse Ausdauerdiagnostik}

\subsubsection{6-Minuten Gehtest}

Der 6-Minuten-Gehtest wurde zu beiden Messzeitpunkten von 79 Probanden abgeschlossen. Von diesen waren 45 in der Kontroll- und 34 in der Trainingsgruppe. Es zeigt sich, dass beide Gruppen signifikante Steigerungen erreicht haben. Die Kontrollgruppe steigerte sich ausgehend von einem Mittelwert von 496m zu T1 auf einen Wert von $527 \mathrm{~m}$ im Mittel zu T2. Es konnte eine Leistungssteigerung von durchschnittlich $31 \mathrm{~m}$ belegt werden. Die Veränderung wurde mit $p=0,032$ als signifikant nachgewiesen.

In der Trainingsgruppe fiel die Leistungssteigerung deutlicher aus. Der Ausgangswert zu T1 war höher als in der Kontrollgruppe und lag im Mittel bei 506m. Zu T2 schafften die Probanden der Trainingsgruppe durchschnittlich 570m, was eine Steigerung von $64 \mathrm{~m}$ bedeutete. Die Veränderung war mit $\mathrm{p}<0,0001$ hochsignifikant. Abbildung 59 und Tabelle 36 zeigen die Verteilung, die Anzahl der Probanden und die Höhe der Leistungssteigerung. Zusätzlich fiel auf, dass sich die Amplitude der Wertestreuung in beiden Gruppen verringerte.

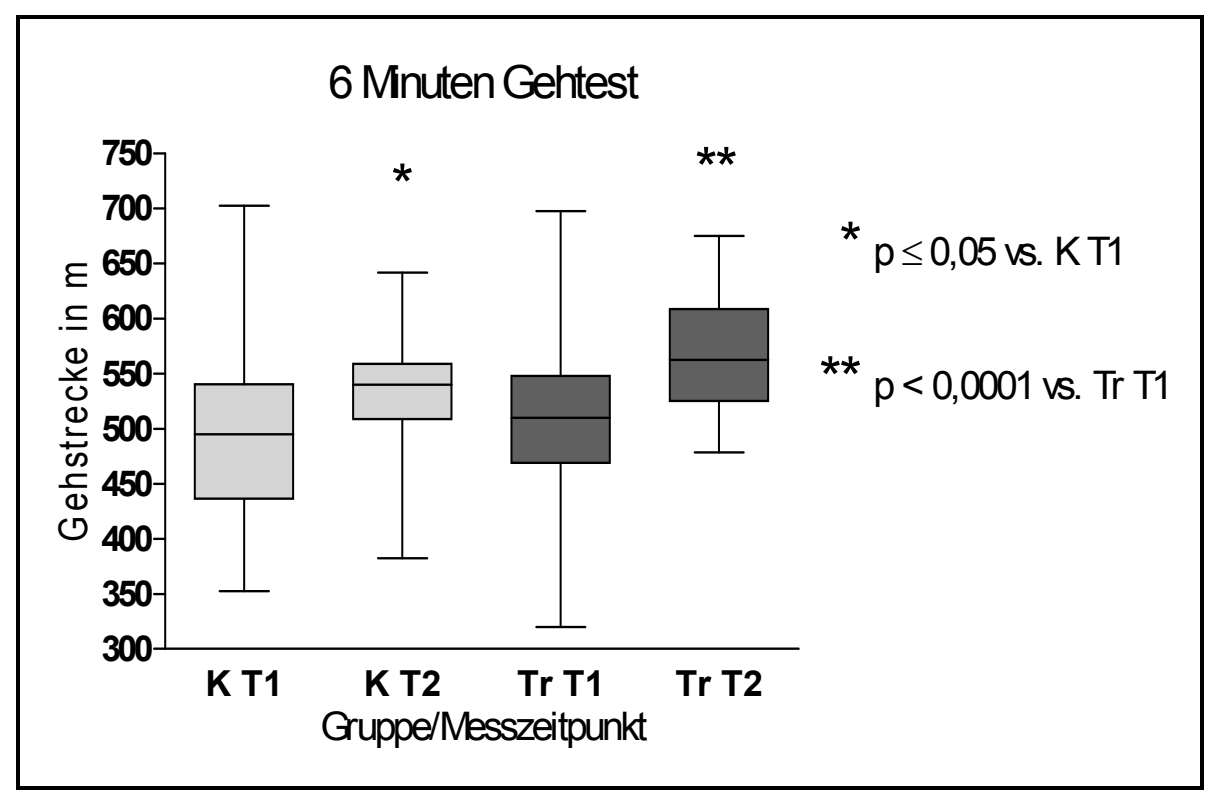

Abbildung 59: 6-Minuten Gehtest Kontrollgruppe und Trainingsgruppe 


\begin{tabular}{|l|r|r|r|r|r|r|}
\hline Gruppe & $\begin{array}{c}\text { Verbesserung } \\
\text { (Absolut/Prozent) }\end{array}$ & $\begin{array}{c}\boldsymbol{\varnothing} \\
\text { in } \mathbf{m}\end{array}$ & $\begin{array}{c}\text { Verschlechterung } \\
\text { (Absolut/ } \\
\text { Prozent) }\end{array}$ & $\begin{array}{c}\boldsymbol{\varnothing} \\
\text { in } \mathbf{~ m}\end{array}$ & $\begin{array}{l}\text { Identisch } \\
\text { (Absolut/ } \\
\text { Prozent) }\end{array}$ & Gesamt \\
\hline $\mathrm{K}$ & $30(68,2 \%)$ & 53,9 & $12(27,3 \%)$ & 46,5 & $2(4,5 \%)$ & 44 \\
\hline $\mathrm{Tr}$ & $26(78,8 \%)$ & 81,8 & $6(18,1 \%)$ & 29,5 & $1(3,1 \%)$ & 33 \\
\hline
\end{tabular}

Tabelle 36: 6-Minuten Gehtest Kontrollgruppe und Trainingsgruppe 


\subsubsection{PWC 130}

\section{Zeitanalysen}

Abbildung 60 stellt die mittlere Dauer des PWC 130 beider Gruppen zu den Messzeitpunkten T1 und T2 dar. Vollständige Tests zu beiden Messzeitpunkten wurden von 70 Probanden absolviert. 43 Probanden waren in der Kontroll- und 27 in der Trainingsgruppe. Es ist erkennbar, dass sich beide Gruppen verbesserten. Die Kontrollgruppe hatte zu T1 im Mittel 9,22 $\pm 2,35$ Minuten bis zur Zielherzfrequenz getreten. Drei Wochen später zu T2 war die durchschnittliche Zeit 9,42 $\pm 2,41$ Minuten. Somit war die Belastung der Kontrollgruppe zu T2 im Mittel 20 Sekunden länger als zu T1.

Die Trainingsgruppe verbesserte sich während der drei Wochen Rehabilitation im Mittel von 8,36 $\pm 2,49$ Minuten auf 9,21 $\pm 2,49$ Minuten. Die Belastung der Trainingsgruppe war zu T2 45 Sekunden länger als zu T1.

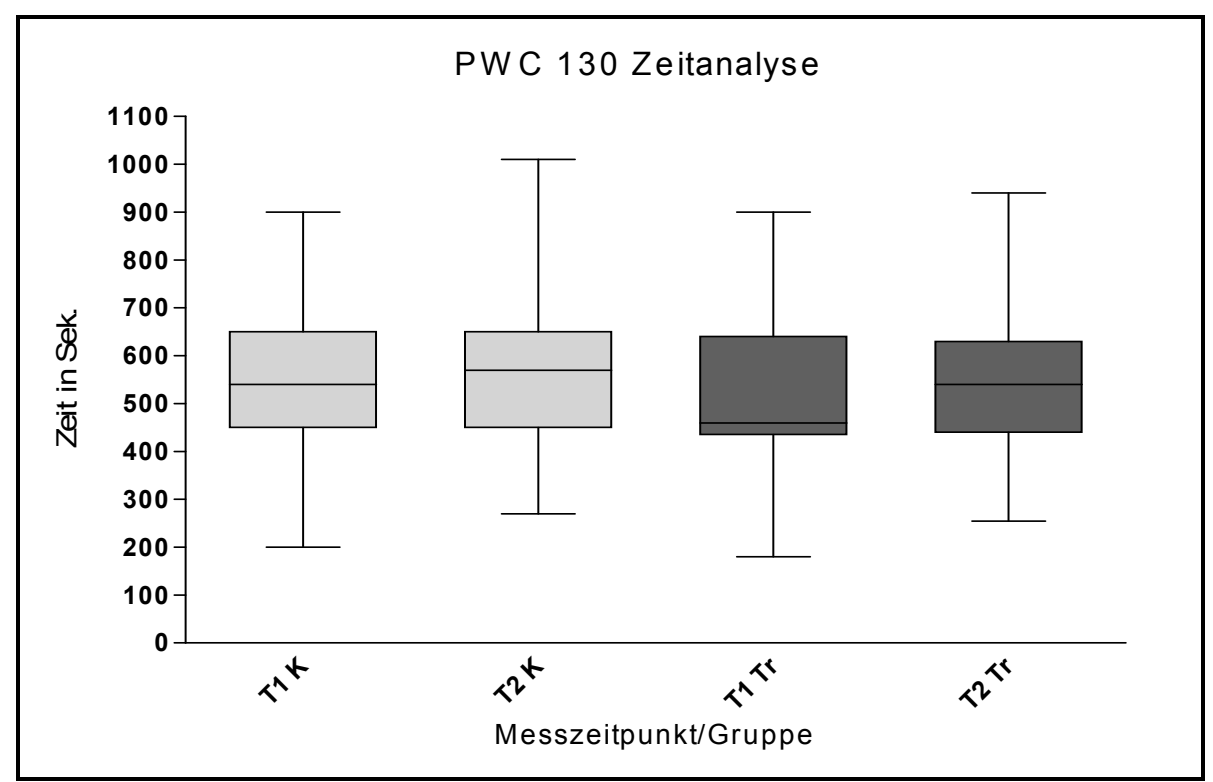

Abbildung 60: PWC 130 Zeitanalyse 
PWC Stufenanalyse

In Abbildung 61 ist die Stufenauswertung des PWC 130 dargestellt. Dabei sind die Stufen dargestellt, in denen die Probanden den Test beendeten. Die exakten prozentualen Werte sind in Tabelle $37 \mathrm{zu}$ finden. Trotz nicht nachweisbarer Signifikanzen war erkennbar, dass eine größere Anzahl von Patienten den Test zu T2 in einer höheren Wattstufe beendete.
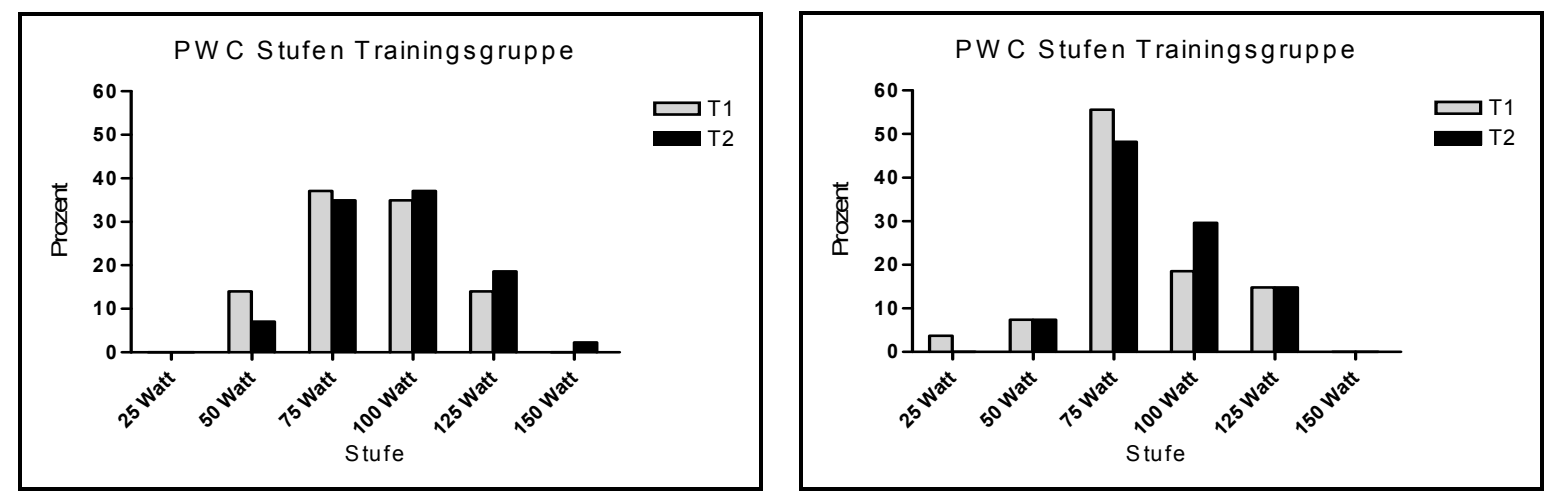

Abbildung 61: PWC 130 Stufen Kontrollgruppe (links) und Trainingsgruppe (rechts)

\section{Kontrollgruppe (43Probanden)}

\begin{tabular}{|l|l|l|l|l|l|l|}
\hline \multicolumn{1}{|c|}{ Stufe } & 25 Watt & 50 Watt & 75 Watt & 100 Watt & 125 Watt & 150 Watt \\
\hline T1 & 0 & $6(14,0 \%)$ & $16(37,1 \%)$ & $15(34,9 \%)$ & $6(14,0 \%)$ & 0 \\
\hline T2 & 0 & $3(7,1 \%)$ & $15(34,9 \%)$ & $16(37,1 \%)$ & $8(18,6 \%)$ & $1(2,3 \%)$ \\
\hline
\end{tabular}

Trainingsgruppe (27 Probanden)

\begin{tabular}{|l|l|l|l|l|l|l|}
\hline T1 & $1(3,7 \%)$ & $2(7,4 \%)$ & $15(55,6 \%)$ & $5(18,5 \%)$ & $4(14,8 \%)$ & 0 \\
\hline T2 & 0 & $2(7,4 \%)$ & $13(48,2 \%)$ & $8(29,6 \%)$ & $4(14,8 \%)$ & 0 \\
\hline
\end{tabular}

Tabelle 37: PWC-Stufenanalyse 


\subsubsection{Ergebnisse Kraftdiagnostik}

Unterscheidung Maximalkraftwerte und Kraftmittelwerte

Die Kraftdiagnostik bestand, wie schon in Abschnitt 5.5.2.3 beschrieben, aus einem isometrischen Maximalkrafttest. Die Testdauer betrug fünf Sekunden. In diesen fünf Sekunden wurde der größte gemessene Kraftwert als Maximalkraftmessergebnis gespeichert. Außerdem wurde aus der Kraftkurve während der Messzeit ein Mittelwert gebildet. Dieser wurde ebenfalls in die Auswertung übernommen und als Kraftmittelwert ausgewertet. Vollständige Messwerte lagen von 57 Probanden vor. Davon waren 33 in der Kontroll- und 24 in der Trainingsgruppe.

Ergebnisse Armkraft

Maximalkraftwerte Arme

Die Abbildung 62 zeigt die Maximalkraftwerte der Arme beider Gruppen. Es wurden die Veränderungen der Maximalkraft zwischen T1 und T2 beider Seiten dargestellt. Es ließ sich in beiden Gruppen eine Steigerung der Kraft nachweisen. Allerdings konnten keine signifikanten Leistungssteigerungen zwischen T1 und T2 ermittelt werden. Die genauen Ergebnisse können Tabelle 38 entnommen werden.
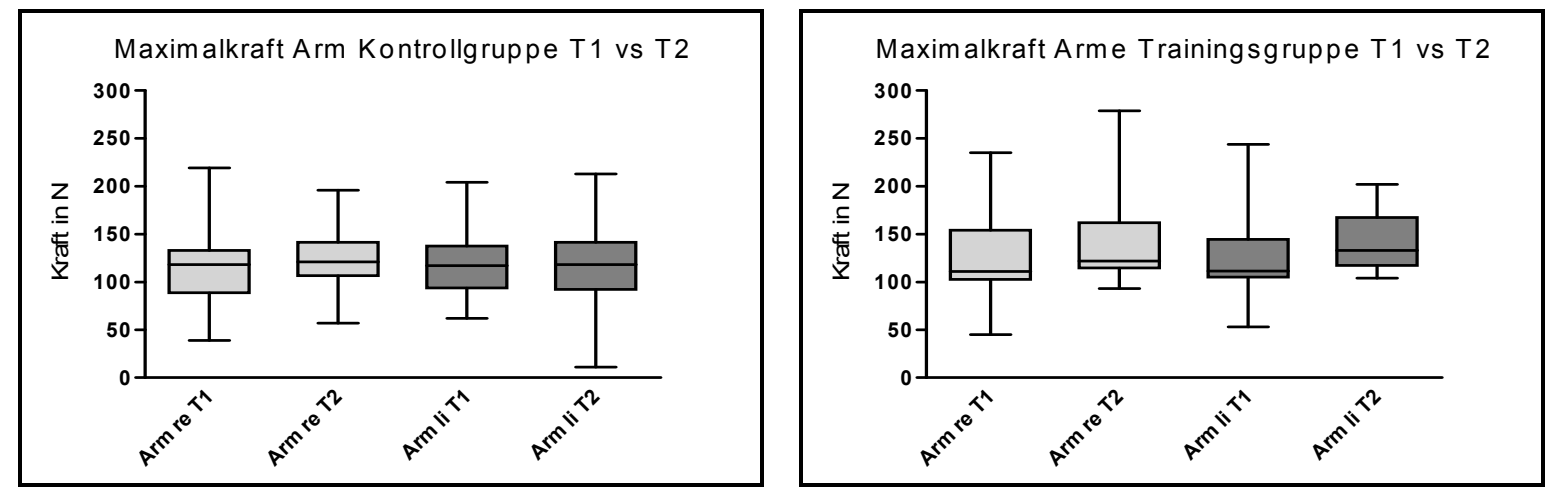

Abbildung 62: Maximalkraftwerte Arme Kontrollgruppe (links) und Trainingsgruppe (rechts) 


\begin{tabular}{|l|r|r|r|r|}
\hline & $\begin{array}{c}\text { Mittelwert T1 } \\
\text { (F in N) }\end{array}$ & $\begin{array}{c}\text { Mittelwert T2 } \\
\mathbf{( F} \text { in } \mathbf{N})\end{array}$ & $\begin{array}{c}\text { Differenz } \\
\mathbf{( F} \text { in } \mathbf{~})\end{array}$ & $\begin{array}{c}\text { Signifikanz (p) } \\
\text { T1 zu T2 }\end{array}$ \\
\hline Kontrollgruppe & & & & 7,9 \\
\hline Arm re. & $114,2 \pm 34,1$ & $122,1 \pm 32,8$ & 2,3 & 0,326 \\
\hline Arm li. & $119,7 \pm 33,3$ & $117,4 \pm 40,0$ & & 0,800 \\
\hline Trainingsgruppe & & & 15,8 & 0,221 \\
\hline Arm re. & $126,4 \pm 40,8$ & $142,2 \pm 44,8$ & 15,6 & 0,201 \\
\hline Arm li. & $127,0 \pm 44,7$ & $142,6 \pm 31,8$ & & \\
\hline
\end{tabular}

Tabelle 38: Maximalkraftwerte Arme

\section{Kraftmittelwerte Arme}

Ein ähnliches Bild zeigen die Ergebnisse für die Kraftmittelwerte der Arme in Abbildung 63. Es ließen sich zwar Leistungssteigerungen nachweisen, allerdings waren die Unterschiede zu gering, um signifikant zu sein. Tabelle 39 zeigt die exakten Werte der Kraftmessungen inklusive der Signifikanzwerte.

Es sei angemerkt, dass sich sowohl die Maximalkraft- als auch die Kraftmittelwerte der Armkraft in der Trainingsgruppe deutlicher verbesserten als in der Kontrollgruppe.
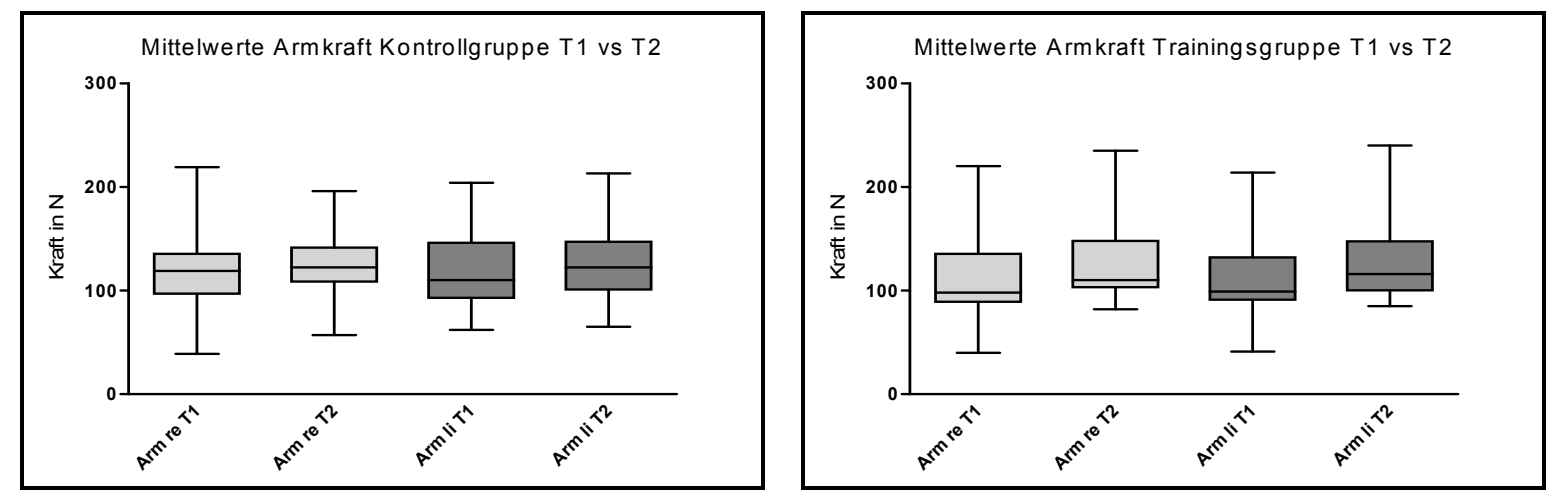

Abbildung 63: Kraftmittelwerte Arme Kontrollgruppe (links) und Trainingsgruppe (rechts) 


\begin{tabular}{|l|r|r|r|r|}
\hline & $\begin{array}{c}\text { Mittelwert T1 } \\
\text { (F in N) }\end{array}$ & $\begin{array}{c}\text { Mittelwert T2 } \\
(\mathbf{F} \text { in } \mathbf{N})\end{array}$ & \multicolumn{1}{|c|}{$\begin{array}{c}\text { Differenz } \\
\mathbf{( F ~ i n ~ N ) ~}\end{array}$} & $\begin{array}{c}\text { Signifikanz (p) } \\
\text { T1 zu T2 }\end{array}$ \\
\hline Kontrollgruppe & & & & \\
\hline Arm re. & $117,6 \pm 33,4$ & $124,9 \pm 31,7$ & 7,3 & 0,399 \\
\hline Arm li. & $118,3 \pm 35,5$ & $124,6 \pm 35,8$ & 6,3 & 0,496 \\
\hline Trainingsgruppe & & & & \\
\hline Arm re. & $111,2 \pm 40,0$ & $125,1 \pm 37,8$ & 13,9 & 0,253 \\
\hline Arm li. & $113,0 \pm 41,7$ & $128,8 \pm 37,4$ & 15,8 & 0,205 \\
\hline
\end{tabular}

Tabelle 39: Kraftmittelwerte Arme

Tabelle 40 zeigt wie viele Probanden aus beiden Gruppen sich während der dreiwöchigen Rehabilitation in ihrer Maximalkraft gesteigert bzw. verschlechtert haben. Zusätzlich ist die durchschnittliche Steigerung oder der Abfall der Maximalkraft in Newton angegeben. Es fiel auf, dass in der Trainingsgruppe der Anteil der Probanden, die während der stationären Rehabilitation ihre Maximalkraft steigern konnten, höher war als in der Kontrollgruppe.

\begin{tabular}{|l|r|r|r|r|r|r|}
\hline Gruppe & $\begin{array}{c}\text { Verbesserung } \\
\text { (Absolut/Prozent) }\end{array}$ & $\begin{array}{c}\varnothing \\
\text { in N }\end{array}$ & $\begin{array}{c}\text { Verschlechterung } \\
\text { (Absolut/Prozent) }\end{array}$ & $\begin{array}{c}\boldsymbol{\varnothing} \\
\text { in N }\end{array}$ & $\begin{array}{c}\text { Identisch } \\
\text { (Absolut/Prozent) }\end{array}$ & Gesamt \\
\hline K & & & & & & $3(9,4)$ \\
\hline Arm li & $17(53,1)$ & 23,1 & $12(37,5)$ & 15,2 & $1(3,1)$ & 32 \\
\hline Arm re & $19(59,4)$ & 25,8 & $12(37,5)$ & 22,5 & & \\
\hline Tr & & & & & $4(0,0)$ & 21 \\
\hline Arm li & $16(76,2)$ & 36,9 & $5(23,8)$ & 43,6 & $1(4,8)$ & 21 \\
\hline Arm re & $16(76,2)$ & 23,3 & $4(19,0)$ & 10,5 & & \\
\hline
\end{tabular}

Tabelle 40: Verteilung der Verbesserung bzw. Verschlechterung der maximalen Armkraft 
Ergebnisse Beinkraft

\section{Maximalkraftwerte Beine}

Bei der Messung der Beinkraft ergab sich ein ähnliches Bild wie bei den Armen. Es konnte nur ein signifikanter Unterschied bei der Kontrollgruppe zwischen der Beinkraft im linken Bein von T1 gegenüber T2 nachgewiesen werden. Alle anderen Ergebnisse wiesen keine signifikanten Unterschiede auf. Die exakten Werte finden sich in Abbildung 64 und Tabelle 41.
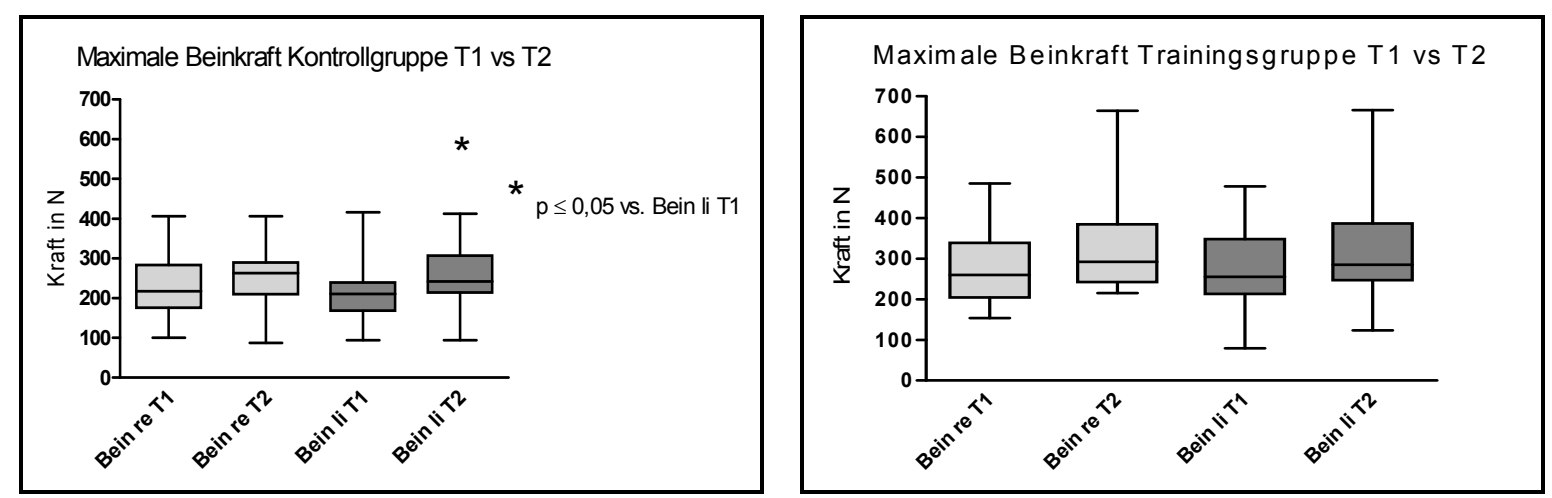

Abbildung 64: Maximalkraftwerte Beine Kontrollgruppe (links) und Trainingsgruppe (rechts)

\begin{tabular}{|l|r|r|r|r|}
\hline & $\begin{array}{c}\text { Mittelwert T1 } \\
\text { (F in N) }\end{array}$ & $\begin{array}{c}\text { Mittelwert T2 } \\
(\mathbf{F} \text { in N) }\end{array}$ & \multicolumn{1}{c|}{$\begin{array}{c}\text { Differenz } \\
(\mathbf{F} \text { in N) }\end{array}$} & $\begin{array}{c}\text { Signifikanz (p) } \\
\text { T1 zu T2 }\end{array}$ \\
\hline Kontrollgruppe & & & & \\
\hline Bein re. & $223,8 \pm 70,7$ & $248,7 \pm 76,0$ & 24,9 & 0,197 \\
\hline Bein li. & $212,8 \pm 72,8$ & $251,7 \pm 77,9$ & 38,9 & 0,045 \\
\hline Trainingsgruppe & & & & \\
\hline Bein re. & $276,9 \pm 92,4$ & $319,8 \pm 111,7$ & 42,9 & 0,183 \\
\hline Bein li. & $274,6 \pm 102,7$ & $323,6 \pm 122,3$ & 49,0 & 0,168 \\
\hline
\end{tabular}

Tabelle 41: Maximalkraftwerte Beine 
Kraftmittelwerte Beine

Bei den Kraftmittelwerten konnten in keiner Gruppe signifikante Unterschiede nachgewiesen werden. Eine Übersicht zu den Kraftmittelwerten der Beine findet sich in Abbildung 65 und Tabelle 42. Trotz fehlender Signifikanz fielen die Verbesserungen in der Trainingsgruppe höher aus als in der Kontrollgruppe.

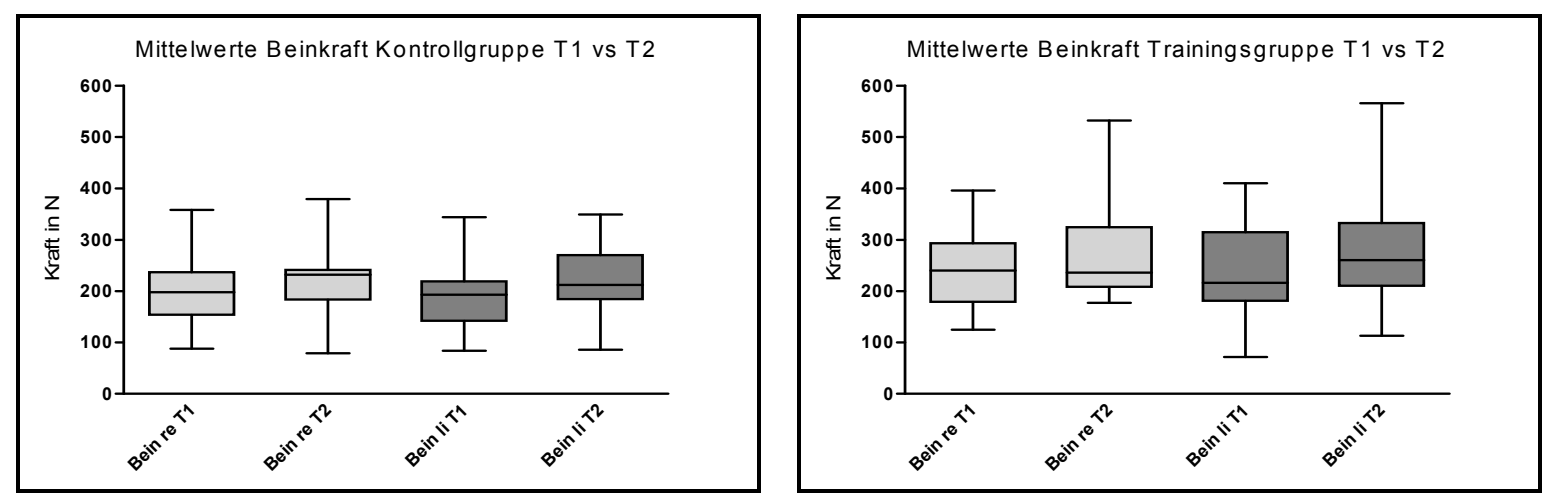

Abbildung 65: Kraftmittelwerte Beine Kontrollgruppe (links) und Trainingsgruppe (rechts)

\begin{tabular}{|l|r|r|r|r|}
\hline & $\begin{array}{c}\text { Mittelwert T1 } \\
\text { (F in N) }\end{array}$ & $\begin{array}{c}\text { Mittelwert T2 } \\
(\mathbf{F} \text { in } \mathbf{N})\end{array}$ & \multicolumn{1}{c|}{$\begin{array}{c}\text { Differenz } \\
(\mathbf{F} \text { in } \mathbf{N})\end{array}$} & $\begin{array}{c}\text { Signifikanz }(\mathbf{p}) \\
\text { T1 zu T2 }\end{array}$ \\
\hline Kontrollgruppe & & & & \\
\hline Bein re. & $201,8 \pm 61,1$ & $216,8 \pm 69,3$ & 15,0 & 0,370 \\
\hline Bein li. & $195,2 \pm 66,5$ & $217,3 \pm 66,7$ & 22,1 & 0,197 \\
\hline Trainingsgruppe & & & & \\
\hline Bein re. & $238,8 \pm 71,3$ & $269,1 \pm 91,6$ & 30,3 & 0,238 \\
\hline Bein li. & $232,4 \pm 91,9$ & $277,3 \pm 109,0$ & 44,9 & 0,157 \\
\hline
\end{tabular}

Tabelle 42: Kraftmittelwerte Beine

Tabelle 43 zeigt die Steigerung bzw. den Abfall der maximalen Beinkraft in Newton. Es war analog zu Tabelle 40 festzustellen, dass in der Trainingsgruppe ein größerer Anteil von Probanden ihre Maximalkraftleistungen steigern konnte. 


\begin{tabular}{|l|r|r|r|r|r|r|}
\hline Gruppe & $\begin{array}{c}\text { Verbesserung } \\
\text { (Absolut/Prozent) }\end{array}$ & \multicolumn{1}{c|}{$\begin{array}{c}\boldsymbol{\sigma} \\
\text { in N }\end{array}$} & $\begin{array}{c}\text { Verschlechterung } \\
\text { (Absolut/Prozent) }\end{array}$ & $\begin{array}{c}\boldsymbol{\emptyset} \\
\text { in N }\end{array}$ & $\begin{array}{c}\text { Identisch } \\
\text { (Absolut/Prozent) }\end{array}$ & Gesamt \\
\hline K & & & & & & \\
\hline Bein li & $18(61,1)$ & 124,6 & $11(39,9)$ & 81,1 & $0(0,0)$ & 29 \\
\hline Bein re & $19(65,5)$ & 92,9 & $10(34,5)$ & 92,5 & $0(0,0)$ & 29 \\
\hline Tr & & & & & & $1(4,5)$ \\
\hline Bein li & $19(86,4)$ & 46,6 & $2(9,1)$ & 23,3 & $1(4,5)$ & 22 \\
\hline Bein re & $18(81,8)$ & 56,6 & $3(13,7)$ & 32,0 & \\
\hline
\end{tabular}

Tabelle 43: Verteilung der Verbesserung bzw. Verschlechterung der maximalen Beinkraft

\subsubsection{Ergebnisse Krafttraining}

Im folgenden Abschnitt werden die Ergebnisse des Krafttrainings anhand der Trainingsprotokolle ausgewertet. Von besonderem Interesse waren Trainingstreue und Motivation der Probanden. Die Häufigkeit und der Umfang des Trainings konnten anhand der Trainingsprotokolle ermittelt werden. Trainingspausen oder Abbruch des Trainings konnten ebenfalls festgestellt werden. Zur Intensität des jeweiligen Trainings konnte aufgrund fehlender Anhaltspunkte keine Aussage getroffen werden. Häufigkeit und Umfang des Trainings wurden zu einer messbaren Größe zusammengefasst.

Der optimale Trainingsumfang wurde als zweimal wöchentliches Training mit der Wiederholungszahl, die das Übungsmanual als Optimum für die jeweilige Übung ausgibt, definiert. Daraus ergab sich eine Berechnungsgrundlage für den optimalen Trainingsumfang. Abbildung 66 gibt eine Herleitung für die Größe des optimalen Trainingsumfangs.

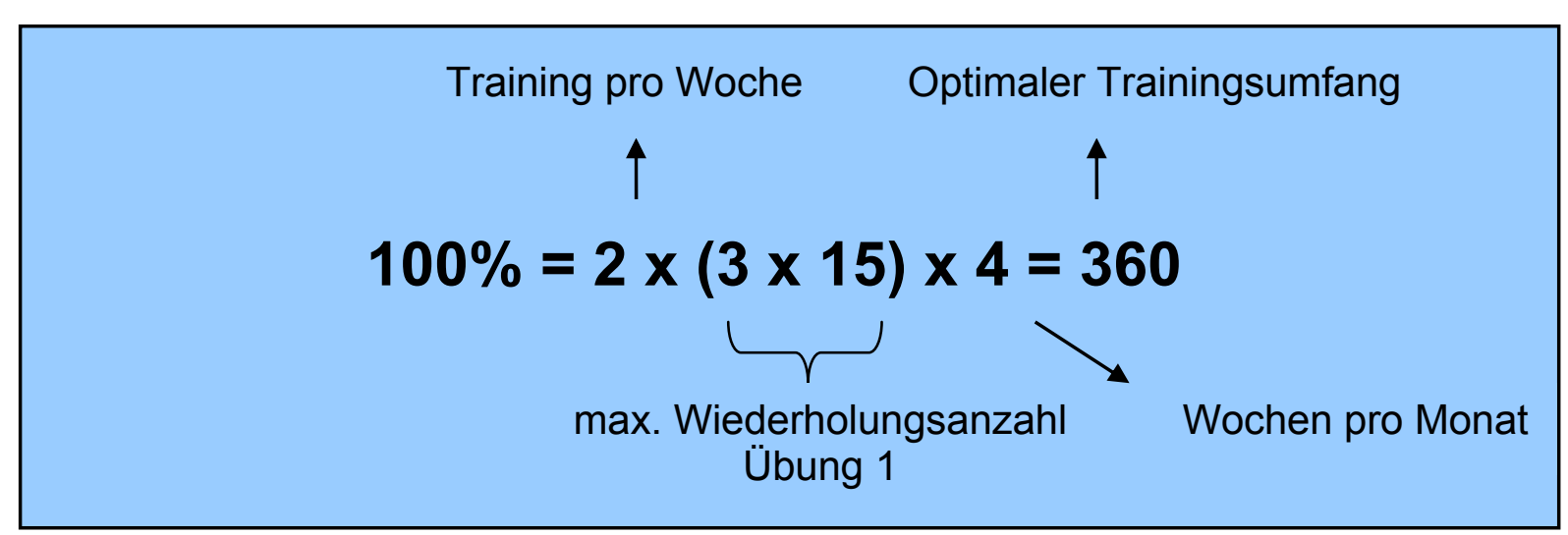

Abbildung 66: Berechnung optimaler Trainingsumfang 
Die Berechnung fand für jede Übung statt. Als Wert für die maximale Wiederholungsanzahl einer Übung wurde der Wert, welcher im Übungsmanual als jeweiliger Optimalwert angegeben wurde, verwendet. Tabelle 44 zeigt die Optimalwerte der einzelnen Übungen. Daraus resultiert die Möglichkeit zu jeder Übung einen prozentualen Wert zu errechnen, der angab, in welchem Maße des optimalen Trainingsumfangs die Übung ausgeführt wurde. Diese Berechnung ist beispielhaft in Abbildung 67 erklärt. Am Ende wurde der Mittelwert aller Umfänge der einzelnen Übungen gebildet, um einen endgültigen Wert für den tatsächlichen Trainingsumfang für jeden Probanden zu erhalten.

\begin{tabular}{|l|c|c|c|c|c|c|c|c|}
\hline Übung & Ü1 & Ü2 & Ü3 & Ü4 & Ü5 & Ü6 & Ü7 & Ü8 \\
\hline $\begin{array}{l}\text { Optimaler } \\
\text { Trainingsumfang }\end{array}$ & $3 \times 15$ & $3 \times 12$ & $3 \times 15$ & $3 \times 10$ & $3 \times 12$ & $3 \times 12$ & $3 \times 10$ & $3 \times 12$ \\
\hline
\end{tabular}

Tabelle 44: Optimaler Trainingsumfang pro Trainingseinheit laut Übungsmanual

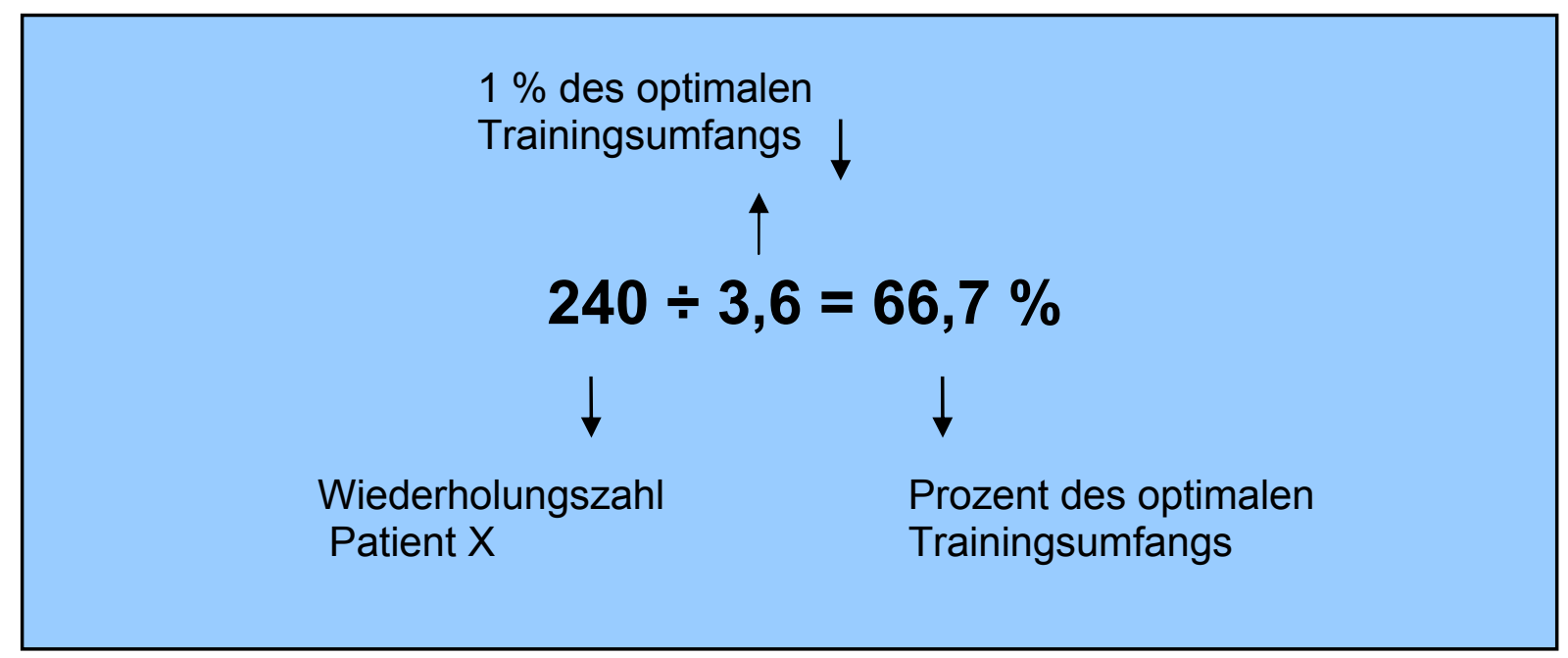

Abbildung 67: Berechnung des tatsächlichen Trainingsumfangs in Prozent des optimalen Trainingsumfangs

Im folgenden Abschnitt werden die Ergebnisse der 28 vollständig eingegangenen Dokumentationsbögen zum Training ausgewertet und beginnend mit einer monatsweisen Auswertung dargestellt. Drei Dokumentationsbögen konnten wegen Unvollständigkeit bei der Auswertung nicht berücksichtigt werden.

Abbildung 68 zeigt die errechneten tatsächlichen Trainingsumfänge der einzelnen Übungen in Prozent des optimalen Trainingsumfangs für alle drei Trainingsmonate. 
Insbesondere die Übungen 4, 6 und 8 reichten nah an den optimalen Trainingsumfang heran. Übung 7 zeigte sogar tatsächliche Werte, die in allen Monaten deutlich über dem optimalen Trainingsumfang lagen. Tabelle 45 zeigt die errechneten Mittelwerte der Wiederholungszahlen, Standardabweichungen und prozentuale Werte des optimalen Trainingsumfangs für alle drei Monate.

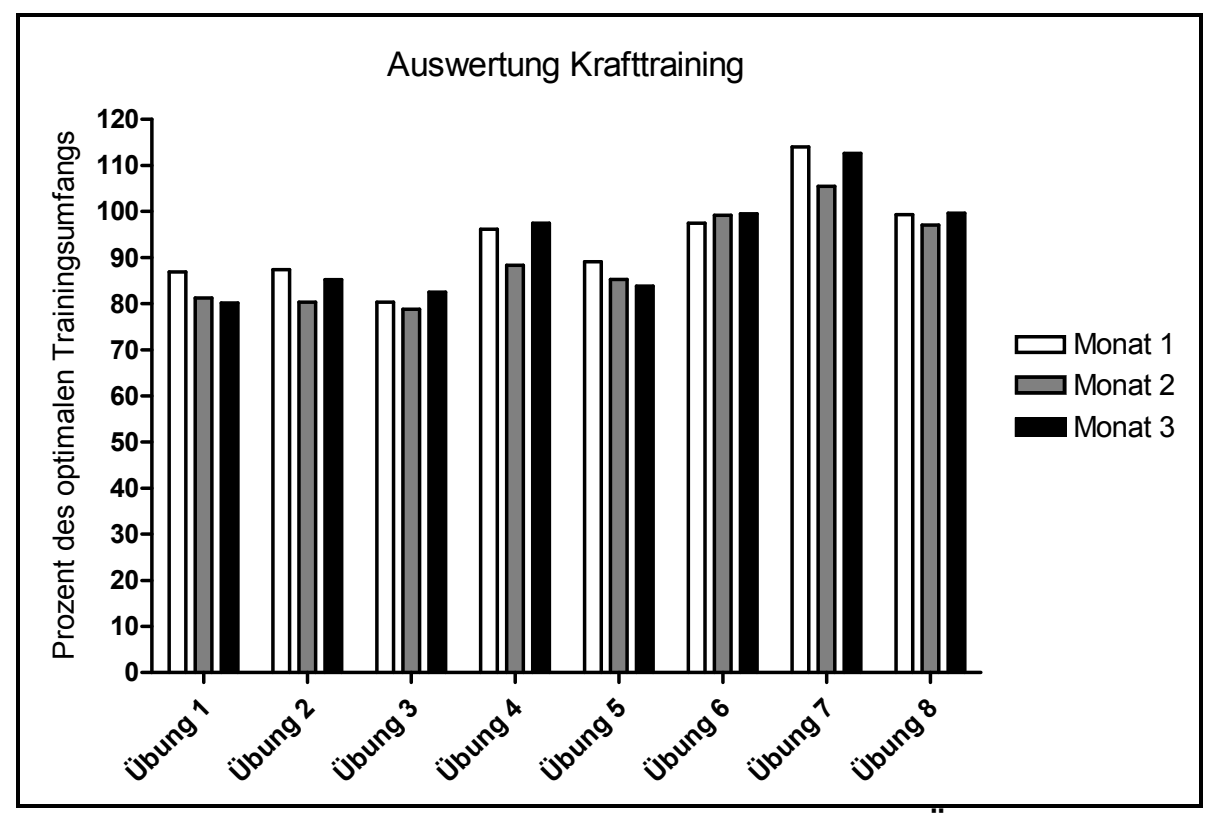

Abbildung 68: Monatsweise Auswertung der einzelnen Übungen

\begin{tabular}{|c|c|c|c|c|c|c|c|c|c|}
\hline & & Ü 1 & Ü 2 & Ü 3 & Ü 4 & Ü 5 & Ü 6 & Ü 7 & Ü 8 \\
\hline \multirow[t]{3}{*}{ 1.Monat } & Mittelwert & 312,6 & 251,8 & 289,1 & 230,7 & 256,7 & 280,8 & 273,8 & 286,0 \\
\hline & Std. Abw. & 159,6 & 173,3 & 173,7 & 157,1 & 142,4 & 193,4 & 186,5 & 165,1 \\
\hline & Prozent & 86,8 & 87,4 & 80,3 & 96,1 & 89,1 & 97,5 & 114,0 & 99,3 \\
\hline \multirow[t]{3}{*}{ 2.Monat } & Mittelwert & 292,4 & 231,3 & 283,7 & 212,0 & 245,6 & 285,7 & 253,1 & 279,5 \\
\hline & Std. Abw. & 161,5 & 152,9 & 221,7 & 144,5 & 138,8 & 177,1 & 157,1 & 177,3 \\
\hline & Prozent & 81,2 & 80,3 & 78,8 & 88,3 & 85,3 & 99,2 & 105,5 & 97,4 \\
\hline \multirow[t]{3}{*}{ 3.Monat } & Mittelwert & 288,5 & 245,4 & 296,9 & 233,9 & 241,4 & 281,7 & 270,3 & 286,9 \\
\hline & Std. Abw. & 186,3 & 168,0 & 260,2 & 165,0 & 166,6 & 202,0 & 183,0 & 195,6 \\
\hline & Prozent & 80,2 & 85,2 & 72,3 & 68,7 & 57,8 & 70,2 & 76,2 & 67,9 \\
\hline
\end{tabular}

Tabelle 45: Monatsweise Auswertung der einzelnen Übungen 
Anschließend wurde die monatsweise Betrachtung auf eine Gesamtbetrachtung der tatsächlichen Trainingsumfänge für jede einzelne Übung reduziert. Die Darstellung erfolgt erneut über den prozentualen Wert des optimalen Trainingsumfangs. Abbildung 69 zeigt die Ergebnisse.

Ähnlich wie in der monatsweisen Betrachtung lagen auch hier die Übungen 6 und 8 sehr nahe am optimalen Trainingsumfang und Übung 7 sogar deutlich darüber. Tabelle 46 zeigt die Mittelwerte der Wiederholungszahlen, die Standardabweichung und die prozentualen Werte vom optimalen Trainingsumfang.

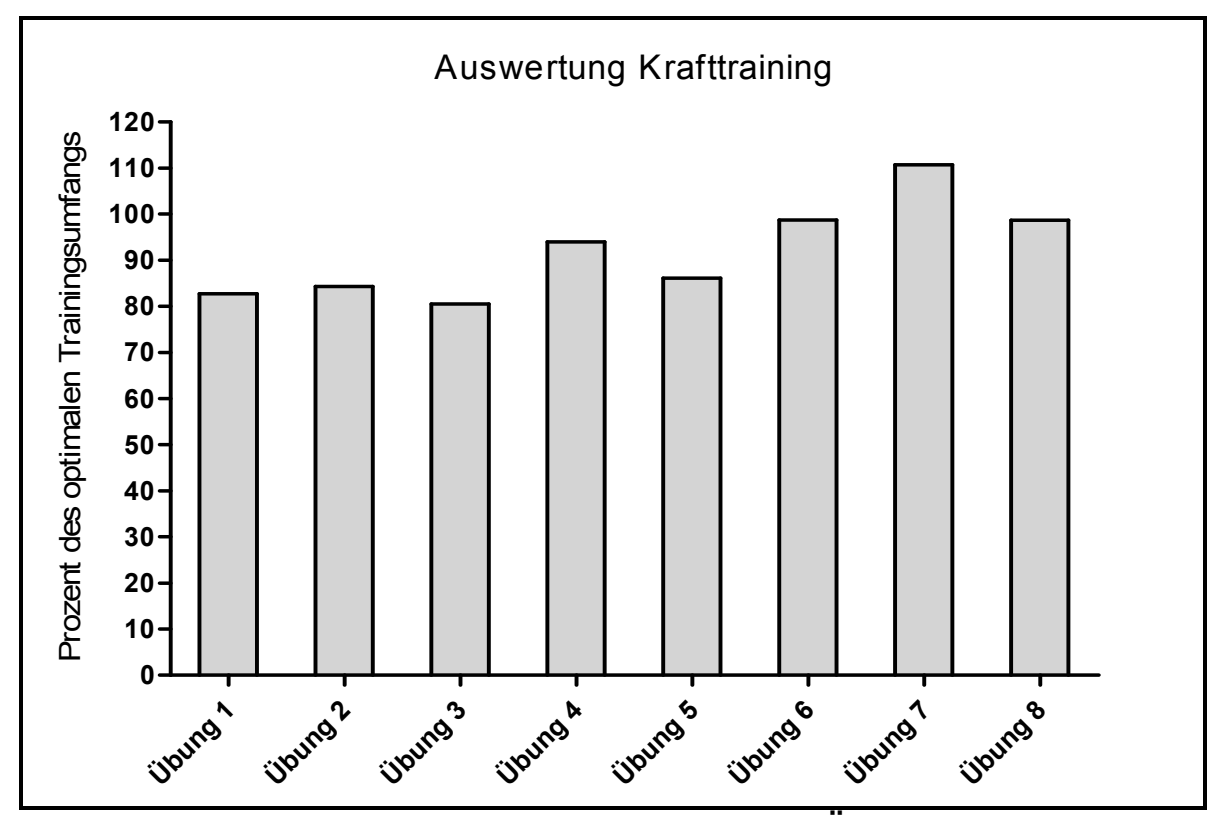

Abbildung 69: Gesamtauswertung der einzelnen Übungen

\begin{tabular}{|l|l|r|r|r|r|r|r|r|r|}
\hline Gesamt & & \multicolumn{1}{|c|}{ Ü 1 } & \multicolumn{1}{c|}{ Ü 2 } & \multicolumn{1}{c|}{ Ü 3 } & \multicolumn{1}{c|}{ Ü 4 } & \multicolumn{1}{c|}{ Ü 5 } & \multicolumn{1}{c|}{ 6 } & Ü 7 & Ü 8 \\
\hline & Mittelwert & 893,6 & 728,5 & 869,8 & 676,6 & 743,7 & 853,2 & 797,0 & 852,4 \\
\hline & Std. Abw. & 442,2 & 413,9 & 600,1 & 414,6 & 382,0 & 517,1 & 444,7 & 465,2 \\
\hline & Prozent & 82,7 & 84,3 & 80,5 & 94,0 & 86,1 & 98,8 & 110,7 & 98,7 \\
\hline
\end{tabular}

Tabelle 46: Gesamtauswertung der einzelnen Übungen

Im letzten Schritt wurde die Betrachtung der tatsächlichen Trainingsumfänge der einzelnen Übungen auf einen einzigen Wert für jeden Patienten reduziert. Der eindeutige Mittelwert des tatsächlichen Trainingsumfangs aller Probanden, aller Übungen und über die gesamte Zeitspanne von drei Monaten liegt bei 90,9\% des optimalen Trainingsumfangs bei einer Standardabweichung von 49,1. 


\subsubsection{Einfluss des tatsächlichen Trainingsumfangs auf verschiedene Untersuchungsparameter}

Im Anschluss an die Auswertung der Dokumentationsbögen zum Krafttraining wurden Subgruppen nach den tatsächlichen Trainingsumfängen gebildet.

Der folgende Abschnitt soll zeigen, inwieweit der tatsächliche Trainingsumfang Einfluss auf verschiedene Parameter von Fatigue, Lebensqualität, Angst und Depression hatte. Im Folgenden werden die Parameter der Subgruppen zu allen Messzeitpunkten dargestellt. Da sich die Auswirkungen des Trainingsprogrammes explizit zu T3 bemerkbar machten, wurde Messzeitpunkt T3 zur Verdeutlichung gesondert dargestellt. Methodisch war dies die einzige Möglichkeit, Ergebnisse des Trainingsprogramms mit den Ergebnissen der Fragebögen in Verbindung zu setzen. Statistisch einwandfreie Korrelationen erschienen aufgrund der enormen Anzahl unterschiedlicher Ausgangsparameter im Patientenkollektiv fragwürdig und nicht praktikabel.

Eine Definition der Subgruppen findet sich in Abbildung 70. Die Subgruppen wurden daraufhin erneut auf Merkmale von Fatigue, Lebensqualität, Angst und Depression zum Messzeitpunkt T3 untersucht. Eine Darstellung der Ergebnisse findet sich im folgenden Abschnitt.

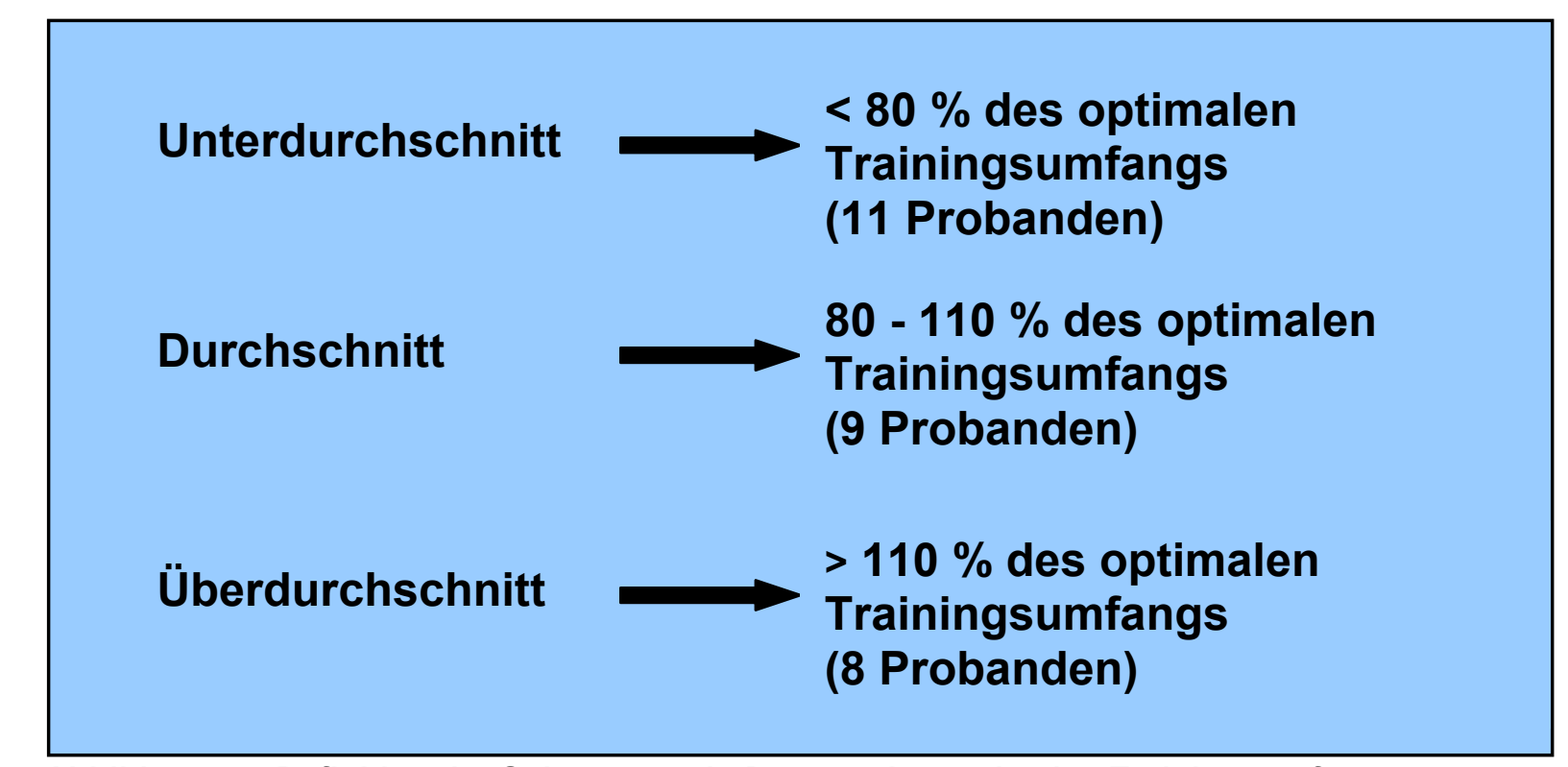

Abbildung 70: Definition der Subgruppen in Prozent des optimalen Trainingsumfangs 
Abbildung 71 zeigt den tatsächlichen Trainingsumfang der Subgruppen in Prozent des optimalen Trainingsumfangs im Vergleich. In Tabelle 47 sind die genauen Zahlen zu finden.

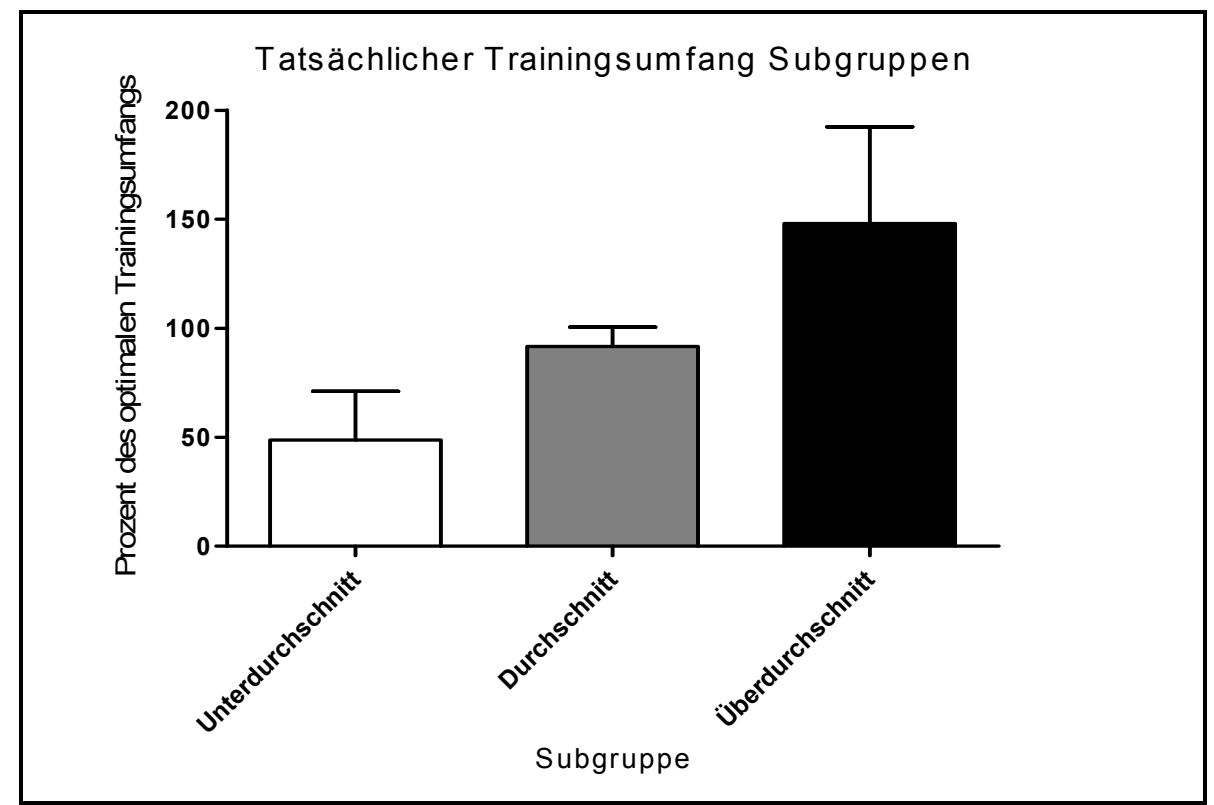

Abbildung 71: Tatsächlicher Trainingsumfang der drei definierten Subgruppen

\begin{tabular}{|l|l|r|r|r|}
\hline Subgruppe & & Unter $\varnothing$ & Durchschnitt & \multicolumn{1}{|c|}{ Über $\varnothing$} \\
\hline & $\begin{array}{l}\text { Mittelwert } \\
\text { (Wiederholungen) }\end{array}$ & 3436 & 6473 & 10444 \\
\hline & Std. Abw. & 1576 & 626 & 3128 \\
\hline & Prozent & 48,7 & 91,7 & 148,0 \\
\hline
\end{tabular}

Tabelle 47: Tatsächlicher Trainingsumfang der drei definierten Subgruppen

\subsubsection{Auswirkungen des tatsächlichen Trainingsumfangs auf den Fatiguerhythmus}

Abbildung 72 zeigt den Fatiguerhythmus in den drei Subgruppen zu allen Messzeitpunkten. Die Abbildung zeigt, dass der nachgewiesene Fatiguerhythmus im Wesentlichen auch in den Subgruppen erkennbar war. Die Fatiguewerte stiegen in allen Subgruppen im Tagesverlauf an. Zudem fällt auf, dass die Fatiguewerte der Subgruppe Unterdurchschnitt zu allen Messzeitpunkten höher waren als die der 
anderen Subgruppen. Durchweg die niedrigsten Fatiguewerte wies die Subgruppe Überdurchschnitt auf. Tabelle 49 gibt die Mittelwerte und Standardabweichung zu Abbildung 72.

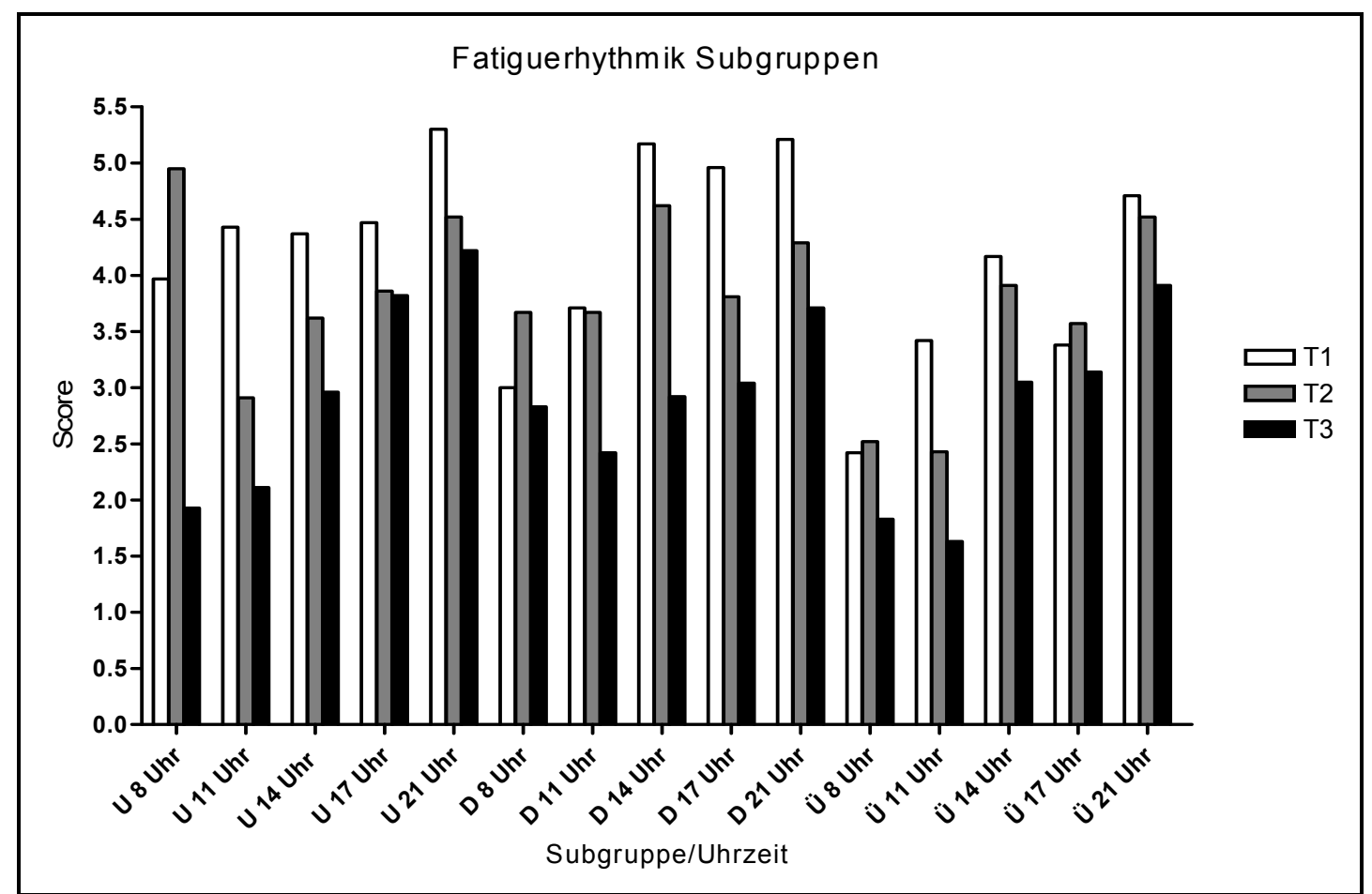

Abbildung 72: Fatiguerhytmik aller Subgruppen (U-Unterdurchschnitt, D-Durchschnitt, ÜÜberdurchschnitt) zu den drei Messzeitpunkten

\begin{tabular}{|l|l|l|l|l|l|}
\hline Unterdurchschnitt & & & & & \\
\hline & $\mathbf{8} \mathbf{~} \mathbf{h r}$ & $\mathbf{1 1} \mathbf{U h r}$ & $\mathbf{1 4} \mathbf{U h r}$ & $\mathbf{1 7} \mathbf{U h r}$ & $\mathbf{2 1} \mathbf{~ h r}$ \\
\hline T1 & $3,97 \pm 2,3$ & $4,43 \pm 1,9$ & $4,37 \pm 1,2$ & $4,47 \pm 1,2$ & $5,30 \pm 2,3$ \\
\hline T2 & $4,95 \pm 2,3$ & $2,91 \pm 1,4$ & $3,62 \pm 1,6$ & $3,86 \pm 2,0$ & $4,52 \pm 2,3$ \\
\hline T3 & $1,93 \pm 1,6$ & $2,11 \pm 1,2$ & $2,96 \pm 1,2$ & $3,82 \pm 2,2$ & $4,22 \pm 2,3$ \\
\hline Durchschnitt & & & & & \\
\hline T1 & $3,00 \pm 2,6$ & $3,71 \pm 2,6$ & $5,17 \pm 2,7$ & $4,96 \pm 2,3$ & $5,21 \pm 2,5$ \\
\hline T2 & $3,67 \pm 3,4$ & $3,67 \pm 2,5$ & $4,62 \pm 2,3$ & $3,81 \pm 2,8$ & $4,29 \pm 2,8$ \\
\hline T3 & $2,83 \pm 2,4$ & $2,42 \pm 2,0$ & $2,92 \pm 2,1$ & $3,04 \pm 2,3$ & $3,71 \pm 2,5$ \\
\hline Überdurchschnitt & & & & & \\
\hline T1 & $2,42 \pm 2,3$ & $3,42 \pm 2,2$ & $4,17 \pm 1,9$ & $3,38 \pm 2,0$ & $4,71 \pm 1,7$ \\
\hline T2 & $2,52 \pm 2,2$ & $2,43 \pm 1,3$ & $3,91 \pm 1,3$ & $3,57 \pm 2,3$ & $4,52 \pm 1,4$ \\
\hline T3 & $1,83 \pm 2,9$ & $1,63 \pm 2,2$ & $3,05 \pm 1,5$ & $3,14 \pm 2,0$ & $3,91 \pm 1,9$ \\
\hline
\end{tabular}

Tabelle 48: Fatiguerhythmik der Subgruppen zu den drei Messzeitpunkten 
Abbildung 73 zeigt, dass in allen Subgruppen die Fatigue zu T3 im Tagesverlauf zunahm. Am Tagesanfang waren die Werte der Gruppe Unterdurchschnitt und Überdurchschnitt niedriger als die Werte der Subgruppe Durchschnitt. Im Tagesverlauf war allerdings erkennbar, dass die Fatiguewerte der Subgruppen Durchschnitt und Überdurchschnitt weniger stark anstiegen und somit niedriger ausfielen als die Werte der Gruppe Unterdurchschnitt. Die Messung um 17 Uhr ergab sogar einen signifikanten Unterschied der Subgruppe Überdurchschnitt gegenüber der Gruppe Unterdurchschnitt. In Tabelle 49 sind die Mittelwerte der Fatigue \pm Standardabweichung inklusive der Signifikanzwerte aller Subgruppen veranschaulicht dargestellt.

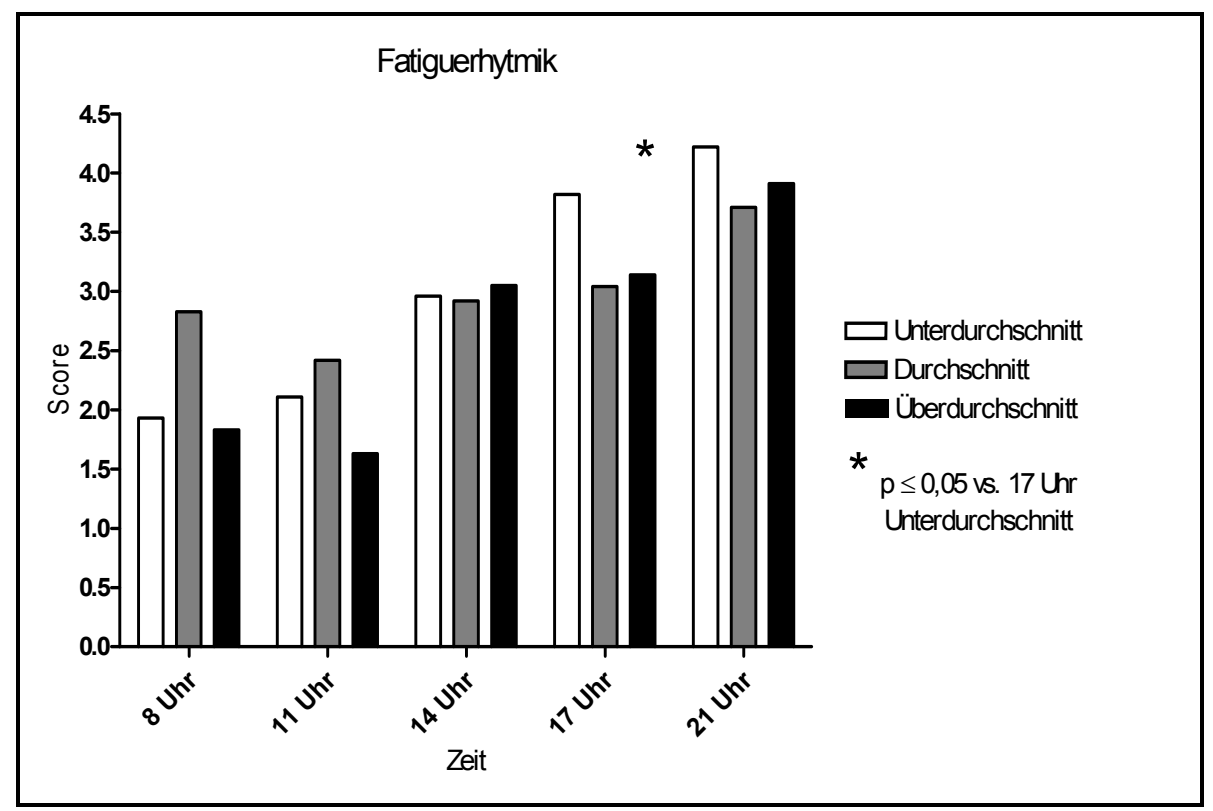

Abbildung 73: Fatiguerhythmikvergleich der Untergruppen zum Messzeitpunkt T3

\begin{tabular}{|l|r|r|r|r|r|}
\hline $\begin{array}{l}\text { Uhrzeit/ } \\
\text { Untergruppe }\end{array}$ & Unter $\varnothing$ & Durchschnitt & Über $\boldsymbol{}$ & $\begin{array}{c}\text { Signifikanz } \\
\text { unter } \varnothing \text { vs. } \\
\text { Durchschnitt }\end{array}$ & $\begin{array}{c}\text { Signifikanz } \\
\text { Durchschnitt } \\
\text { vs. über } \boldsymbol{~}\end{array}$ \\
\hline $\mathbf{8 ~ U h r}$ & $1,93 \pm 1,6$ & $2,83 \pm 2,4$ & $1,83 \pm 2,9$ & 0,0865 & 0,6325 \\
\hline $\mathbf{1 1} \mathbf{U h r}$ & $2,11 \pm 1,2$ & $2,42 \pm 2,0$ & $1,63 \pm 2,2$ & 0,4942 & 0,3004 \\
\hline $\mathbf{1 4} \mathbf{U h r}$ & $2,96 \pm 1,2$ & $2,92 \pm 2,1$ & $3,05 \pm 1,5$ & 0,9228 & 0,6002 \\
\hline $\mathbf{1 7}$ Uhr & $3,82 \pm 2,2$ & $3,04 \pm 2,3$ & $3,14 \pm 2,0$ & 0,2046 & $\mathbf{0 , 0 4 8 6}$ \\
\hline $\mathbf{2 1} \mathbf{U h r}$ & $4,22 \pm 2,3$ & $3,71 \pm 2,5$ & $3,91 \pm 1,9$ & 0,4469 & 0,4192 \\
\hline
\end{tabular}

Tabelle 49: Fatiguerhythmikvergleich der Untergruppen zum Messzeitpunkt T3 


\subsubsection{Auswirkungen des tatsächlichen Trainingsumfangs auf Schlafqualität und Aktivitätslevel}

Abbildung 74 (links) zeigt die Schlafqualität aller Subgruppen in Abhängigkeit vom tatsächlichen Trainingsumfang zu allen Messzeitpunkten. Es wird deutlich, dass die Subgruppe Überdurchschnitt den höchsten Ausgangswert der Schlafqualität hatte. Signifikante Unterschiede zwischen den Messzeitpunkten konnten nur in der Subgruppe Unterdurchschnitt nachgewiesen werden.

Abbildung 74 (rechts) zeigt die Mittelwerte der Schlafqualität der Subgruppen zu T3. Es waren keine großen Unterschiede zwischen den einzelnen Subgruppen

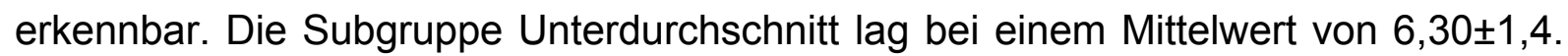
Mit 5,71 $\pm 2,1$ lag die Gruppe Durchschnitt darüber. Die Subgruppe Überdurchschnitt hatte einen Mittelwert von 6,79 $\pm 2,0$.
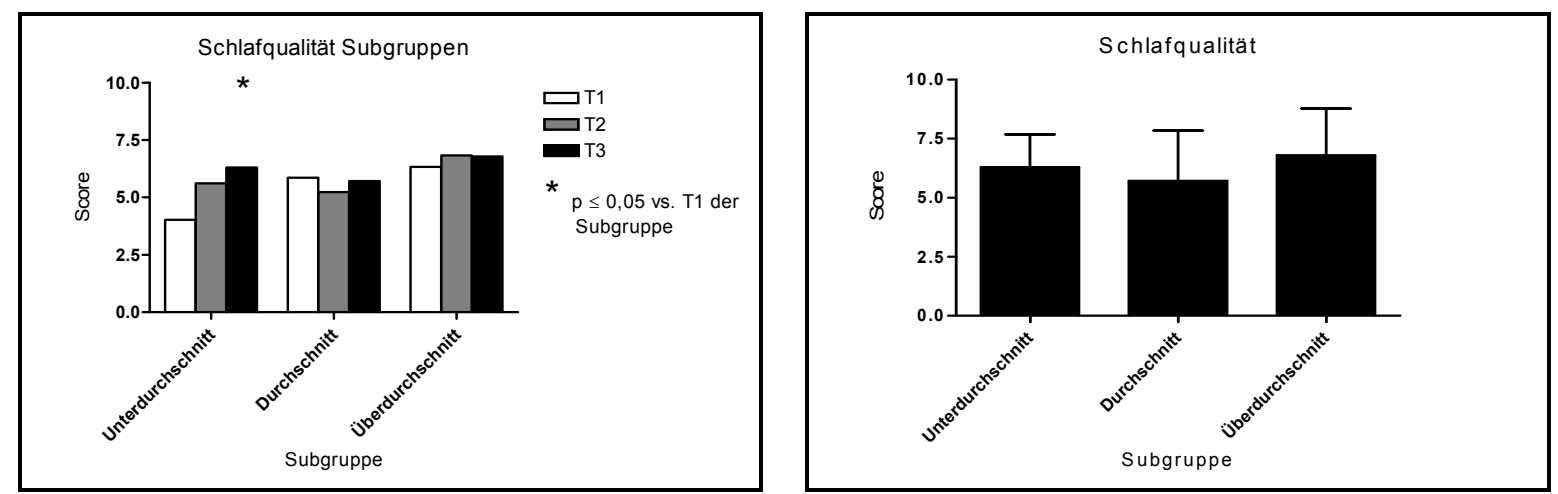

Abbildung 74: Schlafqualität (links) und Schlafqualität zu T3 (rechts) in Abhängigkeit vom tatsächlichen Trainingsumfang

\begin{tabular}{|l|r|r|r|}
\hline & Unterdurchschnitt & Durchschnitt & Überdurchschnitt \\
\hline T1 & $4,03 \pm 1,4$ & $5,86 \pm 2,6$ & $6,33 \pm 1,8$ \\
\hline T2 & $5,61 \pm 1,6$ & $5,24 \pm 1,5$ & $6,83 \pm 1,9$ \\
\hline T3 & $6,30 \pm 1,4$ & $5,71 \pm 2,1$ & $6,79 \pm 2,0$ \\
\hline
\end{tabular}

Tabelle 50: Schlafqualität in Abhängigkeit vom tatsächlichen Trainingsumfang 
In Abbildung 75 (links) ist das Aktivitätslevel der drei Subgruppen zu allen Messzeitpunkten dargestellt. Die Ausgangswerte zu T1 in den Subgruppen waren nahezu identisch. Signifikanzen zwischen den Messzeitpunkten konnten nur in der Subgruppe Überdurchschnitt nachgewiesen werden.

Die Scores lagen in der Gruppe Unterdurchschnitt bei $5,48 \pm 1,4$, in der Gruppe Durchschnitt bei $6,33 \pm 1,7$ und in der Gruppe Überdurchschnitt bei 6,79 $\pm 1,0$. Mit einem Signifikanzwert von $p=0,0005$ war der Unterschied der Subgruppe Unterdurchschnitt gegenüber Überdurchschnitt signifikant.
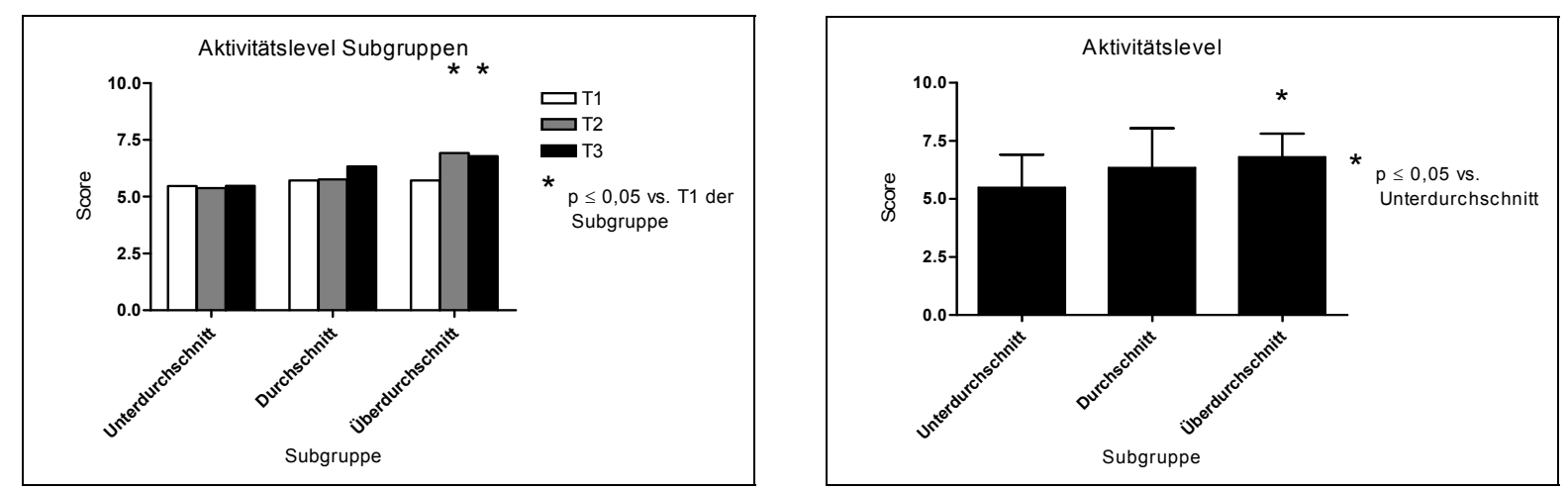

Abbildung 75: Aktivitätslevel (links) und Aktivitätslevel zu T3 (rechts) in Abhängigkeit vom tatsächlichen Trainingsumfang

\begin{tabular}{|l|r|r|r|}
\hline & Unterdurchschnitt & Durchschnitt & Überdurchschnitt \\
\hline T1 & $5,47 \pm 1,5$ & $5,38 \pm 2,6$ & $5,48 \pm 1,4$ \\
\hline T2 & $5,71 \pm 2,0$ & $5,76 \pm 1,6$ & $6,33 \pm 1,7$ \\
\hline T3 & $5,71 \pm 1,7$ & $6,92 \pm 1,1$ & $6,79 \pm 1,0$ \\
\hline
\end{tabular}

Tabelle 51: Aktivitätslevel in Abhängigkeit vom tatsächlichen Trainingsumfang 


\subsubsection{Auswirkungen des tatsächlichen Trainingsumfangs auf Angst und Depression (HADS)}

Abbildung 76 (links) zeigt die Angstwerte der drei Subgruppen zu allen Messzeitpunkten. Es wird deutlich, dass die Subgruppe Unterdurchschnitt zu T1 höhere Ausgangswerte hatte als die anderen beiden Subgruppen. Allerdings konnten auch nur bei der Subgruppe Unterdurchschnitt signifikante Veränderungen zwischen den Messzeitpunkten nachgewiesen werden.

Die Subgruppe Unterdurchschnitt lag zu T3 bei einem Mittelwert von 6,40 $\pm 3,6$. In der

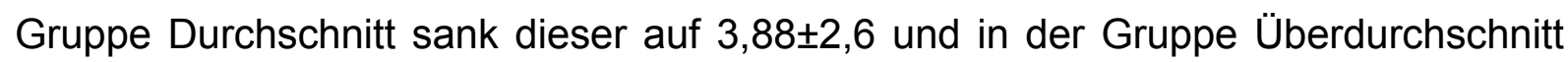
auf 3.00 $\pm 2,7$. Ein signifikanter Unterschied der Gruppe Unterdurchschnitt gegenüber der Gruppe Überdurchschnitt lag mit einem Signifikanzwert von $p=0,0431$ vor.
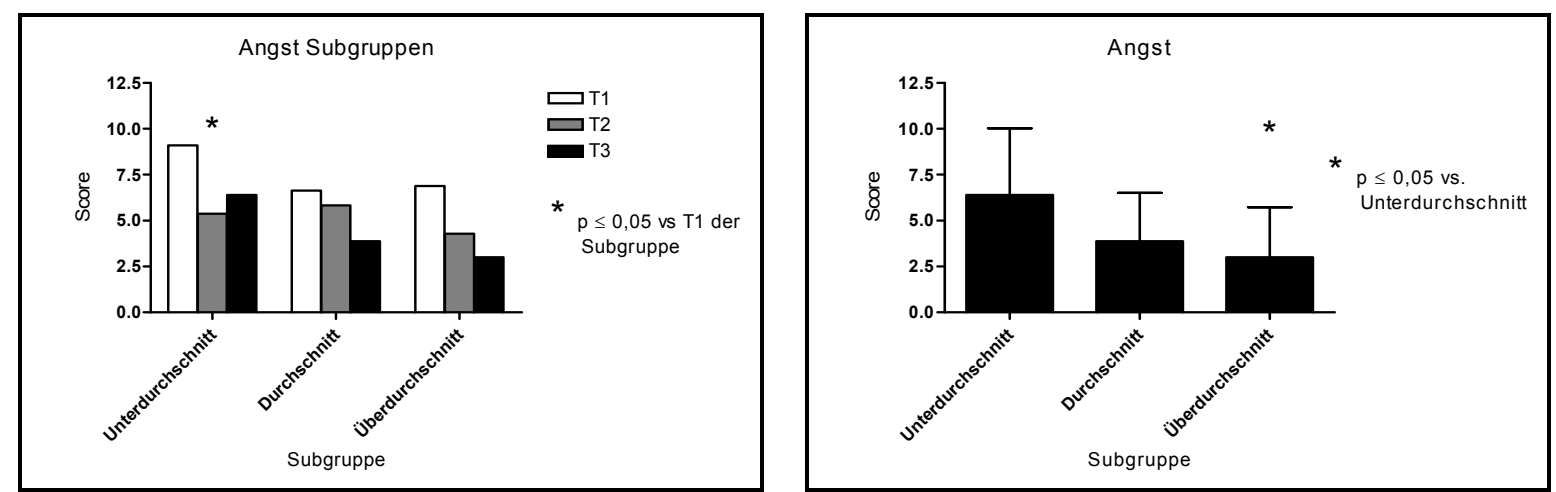

Abbildung 76: Angst zu allen Messzeitpunkten (links) und Angst zu T3 (rechts) in Abhängigkeit vom tatsächlichen Trainingsumfang

\begin{tabular}{|l|r|r|r|}
\hline & Unterdurchschnitt & Durchschnitt & Überdurchschnitt \\
\hline T1 & $9,10 \pm 3,2$ & $6,63 \pm 3,9$ & $6,88 \pm 4,6$ \\
\hline T2 & $5,38 \pm 2,4$ & $5,83 \pm 3,5$ & $4,29 \pm 3,4$ \\
\hline T3 & $6,40 \pm 3,6$ & $3,88 \pm 2,6$ & $3,00 \pm 2,9$ \\
\hline
\end{tabular}

Tabelle 52: Angst in Abhängigkeit vom tatsächlichen Trainingsumfang

Abbildung 77 (links) zeigt die Depressionswerte der drei Subgruppen zu allen Messzeitpunkten. Es ist erneut erkennbar, dass der Ausgangswert der Subgruppe Unterdurchschnitt höher lag als bei den anderen Subgruppen. Signifikante Effekte ließen sich nur in der Subgruppe Überdurchschnitt nachweisen. 
In Abbildung 77 (rechts) ist die Subskala Depression zu T3 dargestellt. Erneut lag

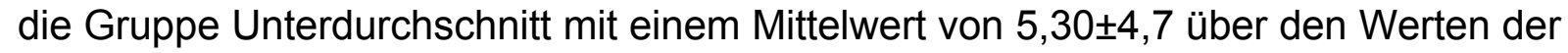

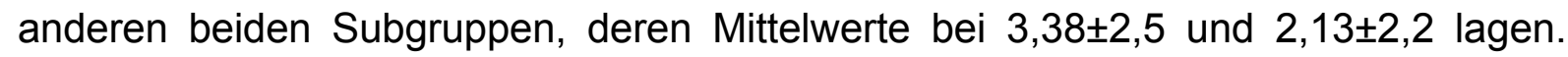
Signifikant unterschiedlich waren die Subgruppen jedoch nicht.
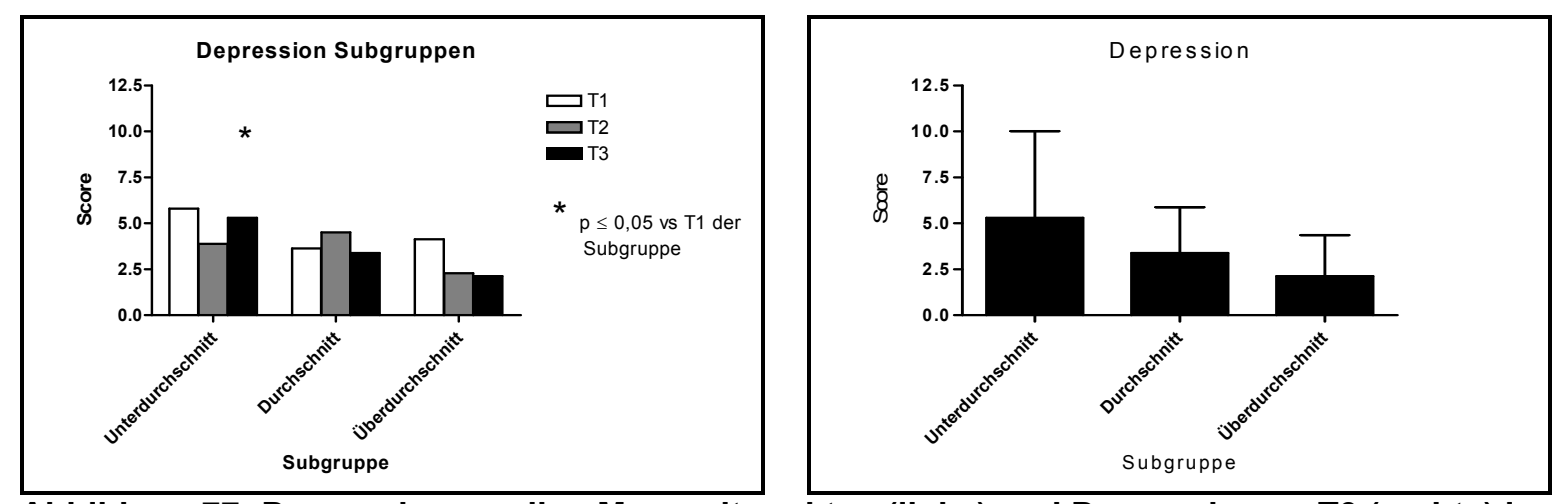

Abbildung 77: Depression zu allen Messzeitpunkten (links) und Depression zu T3 (rechts) in Abhängigkeit vom tatsächlichen Trainingsumfang

\begin{tabular}{|l|r|r|r|}
\hline & Unterdurchschnitt & Durchschnitt & Überdurchschnitt \\
\hline T1 & $5,80 \pm 2,7$ & $3,63 \pm 2,1$ & $4,16 \pm 3,0$ \\
\hline T2 & $3,86 \pm 2,4$ & $4,50 \pm 2,5$ & $2,29 \pm 2,2$ \\
\hline T3 & $5,30 \pm 4,7$ & $3,36 \pm 2,5$ & $2,13 \pm 2,2$ \\
\hline
\end{tabular}

Tabelle 53: Depression in Abhängigkeit vom tatsächlichen Trainingsumfang 


\subsubsection{Auswirkungen des tatsächlichen Trainingsumfangs auf verschiedene Subskalen des MFI}

Im diesem Abschnitt werden verschiedene Subskalen des MFI für die oben gebildeten Subgruppen untersucht.

Abbildung 78 (links) zeigt eine Darstellung der Allgemeinen Fatigue für die drei Subgruppen zu allen Messzeitpunkten. Die Ausgangswerte der einzelnen Gruppen zu T1 unterschieden sich nur geringfügig. Signifikante Veränderungen zwischen den Messzeitpunkten konnten in allen Subgruppen nachgewiesen werden.

Die Gruppe Überdurchschnitt hatte zu T3 mit 25,00 18,9 einen niedrigeren Wert als

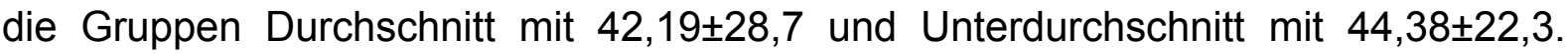
Signifikante Unterschiede zwischen den Subgruppen lagen nicht vor.
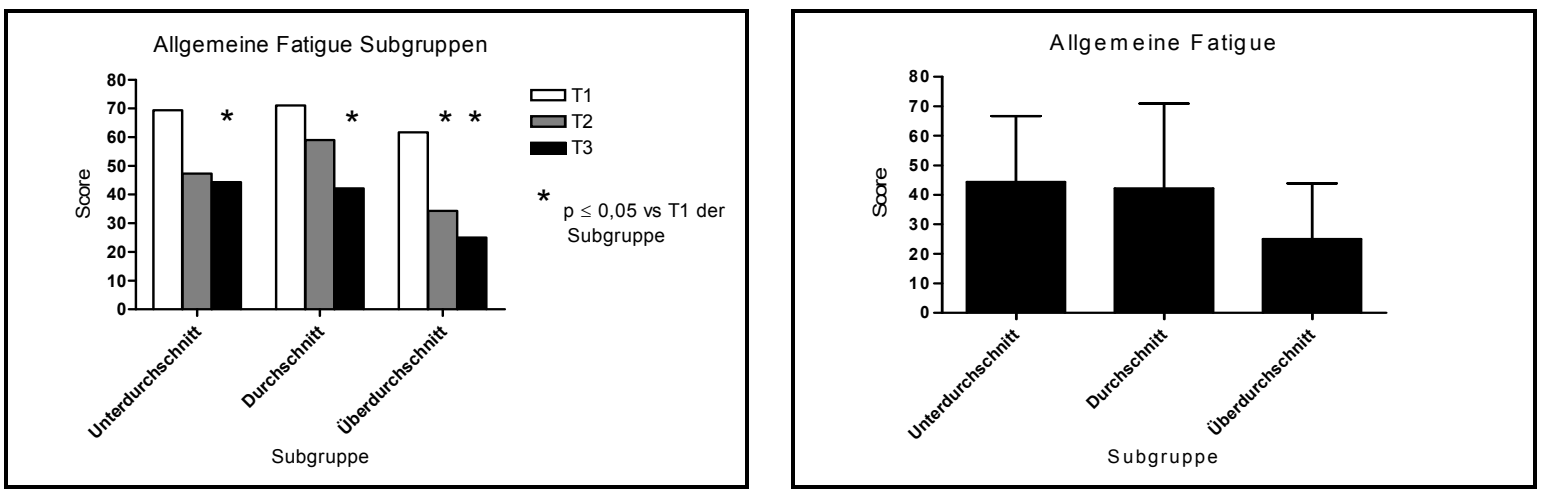

Abbildung 78: Allgemeine Fatigue zu allen Messzeitpunkten (links) und Allgemeine Fatigue zu T3 (rechts) in Abhängigkeit vom

\begin{tabular}{|l|r|r|r|}
\hline & Unterdurchschnitt & Durchschnitt & Überdurchschnitt \\
\hline T1 & $69,38 \pm 15,2$ & $71,09 \pm 22,6$ & $61,72 \pm 27,8$ \\
\hline T2 & $47,32 \pm 28,2$ & $59,82 \pm 28,8$ & $34,28 \pm 17,7$ \\
\hline T3 & $44,38 \pm 22,3$ & $42,19 \pm 28,7$ & $25,00 \pm 18,9$ \\
\hline
\end{tabular}

Tabelle 54: Allgemeine Fatigue in Abhängigkeit vom tatsächlichen Trainingsumfang

Abbildung 79 (links) zeigt die Subskala Physische Fatigue der drei Subgruppen zu allen Messzeitpunkten. Zu T1 war der Ausgangswert in der Subgruppe Unterdurchschnitt höher als in den anderen beiden Subgruppen. Signifikante Veränderungen zwischen den Messzeitpunkten konnten in den Subgruppen Unterdurchschnitt und Überdurchschnitt nachgewiesen werden. 
Die Subskala Physische Fatigue, in Abbildung 79 (rechts), zeigte zu T3 signifikante Unterschiede zwischen der Subgruppe Unterdurchschnittlich mit 45,00 $\pm 15,3$ und der Gruppe Überdurchschnittlich mit 26,56 $\pm 20,0$. Der Signifikanzwert lag bei $p=0,0409$. Mit einem Mittelwert von 38,28 $\pm 30,9$ lag die Subgruppe Durchschnitt zwischen den beiden anderen.
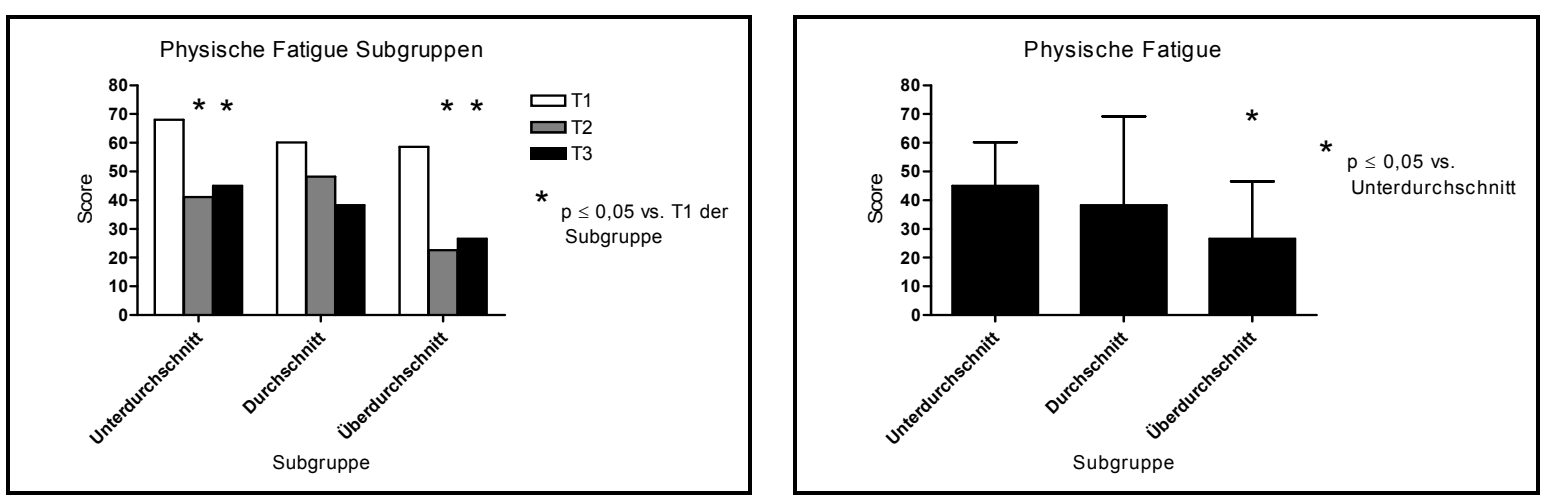

Abbildung 79: Physische Fatigue zu allen Messzeitpunkten (links) und Physische Fatigue zu T3 (rechts) in Abhängigkeit vom tatsächlichen Trainingsumfang

\begin{tabular}{|l|r|r|r|}
\hline & Unterdurchschnitt & Durchschnitt & Überdurchschnitt \\
\hline T1 & $68,06 \pm 14,1$ & $60,19 \pm 28,3$ & $58,59 \pm 22,6$ \\
\hline T2 & $41,07 \pm 21,9$ & $48,21 \pm 36,2$ & $22,66 \pm 20,9$ \\
\hline T3 & $45,00 \pm 15,2$ & $38,28 \pm 30,9$ & $26,56 \pm 20,0$ \\
\hline
\end{tabular}

Tabelle 55: Physische Fatigue in Abhängigkeit vom tatsächlichen Trainingsumfang 
In Abbildung 80 (links) ist die Subskala Mentale Fatigue der drei Subgruppen zu allen Messzeitpunkten dargestellt. Es zeigte sich, dass der Ausgangswert zu T1 in der Subgruppe Unterdurchschnitt höher war als in den anderen beiden Subgruppen. Der T1 Wert der Subgruppe Überdurchschnitt war am niedrigsten.

Zu T3 konnten keine signifikanten Unterschiede zwischen den Subgruppen festgestellt werden. Die Subgruppe Unterdurchschnitt lag bei einem Mittelwert von $37,50 \pm 27,9$, die Subgruppe Durchschnitt bei $30,47 \pm 23,7$ und die Subgruppe Überdurchschnitt lag bei 18,75 $\pm 23,6$.
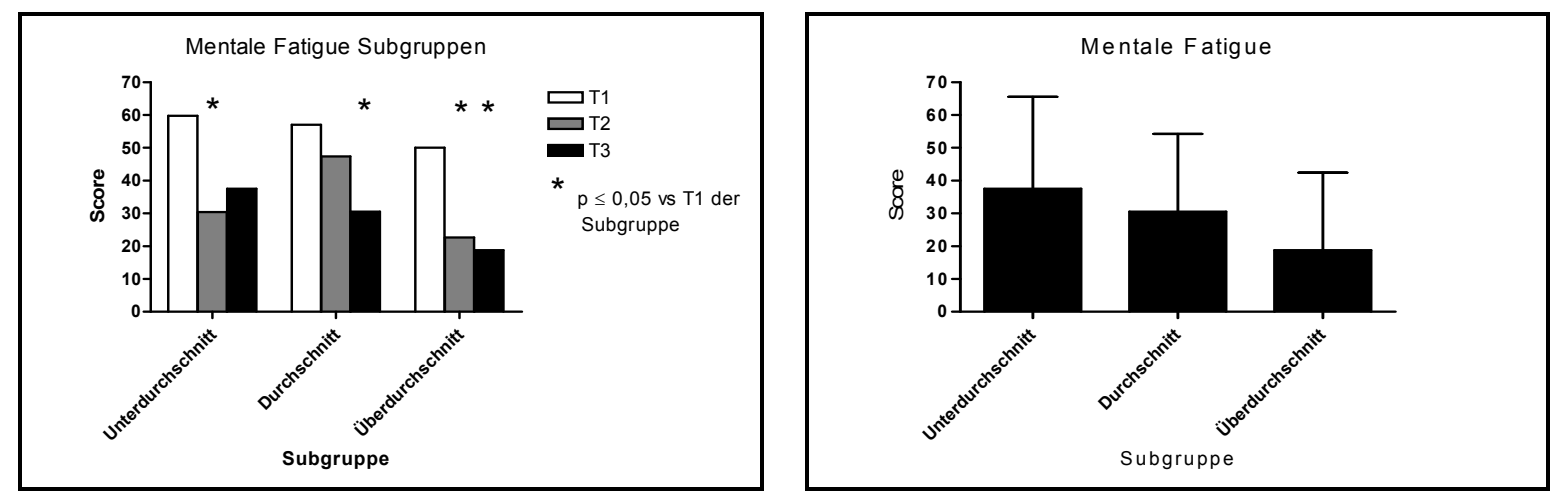

Abbildung 80: Mentale Fatigue zu allen Messzeitpunkten (links) und Mentale Fatigue zu T3 (rechts) in Abhängigkeit vom tatsächlichen Trainingsumfang

\begin{tabular}{|l|r|r|r|}
\hline & Unterdurchschnitt & Durchschnitt & Überdurchschnitt \\
\hline T1 & $59,72 \pm 21,0$ & $57,03 \pm 21,0$ & $50,00 \pm 28,7$ \\
\hline T2 & $30,36 \pm 30,3$ & $47,32 \pm 23,6$ & $22,66 \pm 18,9$ \\
\hline T3 & $37,50 \pm 28,0$ & $30,47 \pm 23,7$ & $18,75 \pm 23,6$ \\
\hline
\end{tabular}

Tabelle 56: Mentale Fatigue in Abhängigkeit vom tatsächlichen Trainingsumfang 
In Abbildung 81 (links) ist die Auswertung der Subskala Reduzierte Aktivität der drei Subskalen zu allen Messzeitpunkten dargestellt. Die Ausgangswerte zu T1 waren ähnlich. Signifikante Unterschiede zwischen den Messzeitpunkten konnten nur in der Subgruppe Überdurchschnitt nachgewiesen werden.

Die Subgruppe Unterdurchschnitt lag zu T3 mit einem Mittelwert von 40,00 23,4 am höchsten. Mit 34,38 $\pm 25,9$ lag die Gruppe Durchschnitt darunter. Die Subgruppe Überdurchschnitt lag mit einem Mittelwert von 26,56 $\pm 16,3$ unter der Gruppe Unterdurchschnitt.
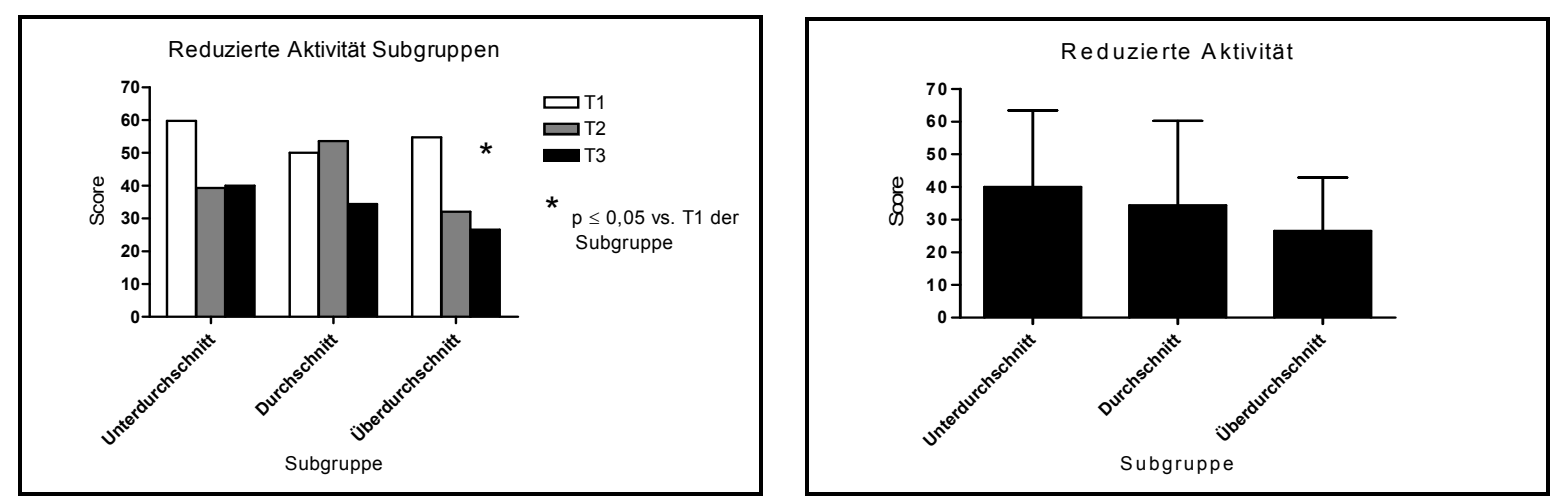

Abbildung 81: Reduzierte Aktivität zu allen Messzeitpunkten (links) und Reduzierte Aktivität zu T3 (rechts) in Abhängigkeit vom tatsächlichen Trainingsumfang

\begin{tabular}{|l|r|r|r|}
\hline & Unterdurchschnitt & Durchschnitt & Überdurchschnitt \\
\hline T1 & $59,72 \pm 20,5$ & $50,00 \pm 29,9$ & $54,69 \pm 23,8$ \\
\hline T2 & $39,29 \pm 28,6$ & $53,57 \pm 35,7$ & $32,03 \pm 19,9$ \\
\hline T3 & $40,00 \pm 23,4$ & $34,38 \pm 25,9$ & $26,56 \pm 16,3$ \\
\hline
\end{tabular}

Tabelle 57: Reduzierte Aktivität in Abhängigkeit vom tatsächlichen Trainingsumfang

Abbildung 82 (links) zeigt die Subskala Reduzierte Motivation aller Subgruppen zu den drei Messzeitpunkten. Erneut zeigte sich in der Subgruppe Unterdurchschnitt ein höherer Ausgangswert zu T1 als in den anderen beiden Gruppen. Signifikante Veränderungen innerhalb der Subgruppen ließen sich zu keinem Messzeitpunkt nachweisen. Abbildung 82 (rechts) zeigt die Subskala zu T3. Die Subgruppe Durchschnitt lag mit einem Mittelwert von 10,94 $\pm 8,0$ am niedrigsten. Dieser war mit 
einem Signifikanzwert von $\mathrm{p}=0,0094$ signifikant gegenüber der Subgruppe

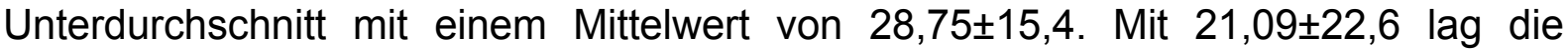
Subgruppe Überdurchschnitt zwischen den beiden anderen.
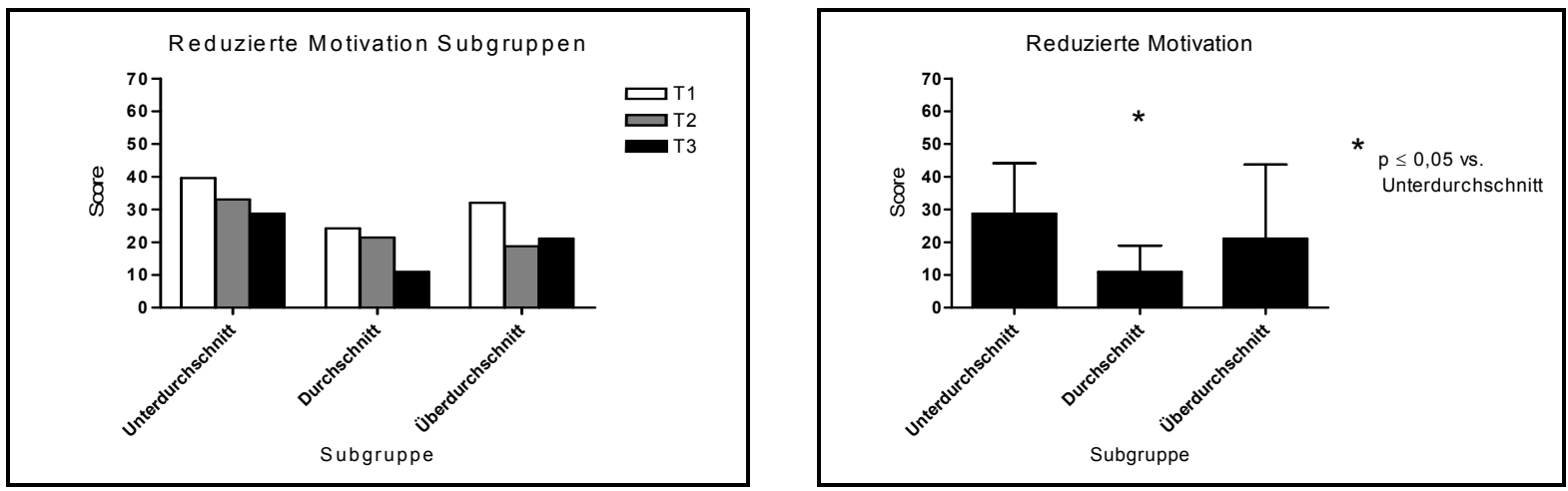

Abbildung 82: Reduzierte Motivation zu allen Messzeitpunkten (links) und Reduzierte Motivation zu T3 (rechts) in Abhängigkeit vom tatsächlichen Trainingsumfang

\begin{tabular}{|l|r|r|r|}
\hline & Unterdurchschnitt & Durchschnitt & Überdurchschnitt \\
\hline T1 & $39,58 \pm 18,5$ & $24,22 \pm 15,8$ & $32,03 \pm 19,0$ \\
\hline T2 & $33,04 \pm 16,0$ & $21,43 \pm 17,3$ & $18,75 \pm 16,3$ \\
\hline T3 & $28,75 \pm 15,4$ & $10,94 \pm 8,0$ & $21,09 \pm 22,6$ \\
\hline
\end{tabular}

Tabelle 58: Reduzierte Motivation in Abhängigkeit vom tatsächlichen Trainingsumfang 


\subsubsection{Auswirkungen des tatsächlichen Trainingsumfangs auf verschiedene Subskalen des EORTC QLQ-C30}

Es sei erneut darauf hingewiesen, dass die Subskalen des EORTC QLQ-C30, wie schon in Abschnitt 6.2.11, in Funktionsskalen und Symptomskalen unterschieden werden. Hierauf gilt es bei der Interpretation zu achten.

Abbildung 83 (links) zeigt die Funktionsskala Physical Functioning der drei Subgruppen zu allen Messzeitpunkten. Lediglich in der Subgruppe Unterdurchschnitt traten signifikante Veränderungen zwischen den Messzeitpunkten auf. Die Ausgangswerte zu T1 waren in der Subgruppe Überdurchschnitt am höchsten.

Abbildung 83 (links) zeigt, dass die Mittelwerte zu T3 ausgehend von der Subgruppe Unterdurchschnitt mit 79,33 $\pm 13,5$ stetig anstiegen. Die Subgruppe Durchschnitt wies einen Wert von $85,00 \pm 12,7$ auf und die Gruppe Überdurchschnitt lag bei $91,67 \pm 7,8$ und war damit signifikant gegenüber der Subgruppe Unterdurchschnitt. Der Signifikanzwert lag bei $p=0,0359$.
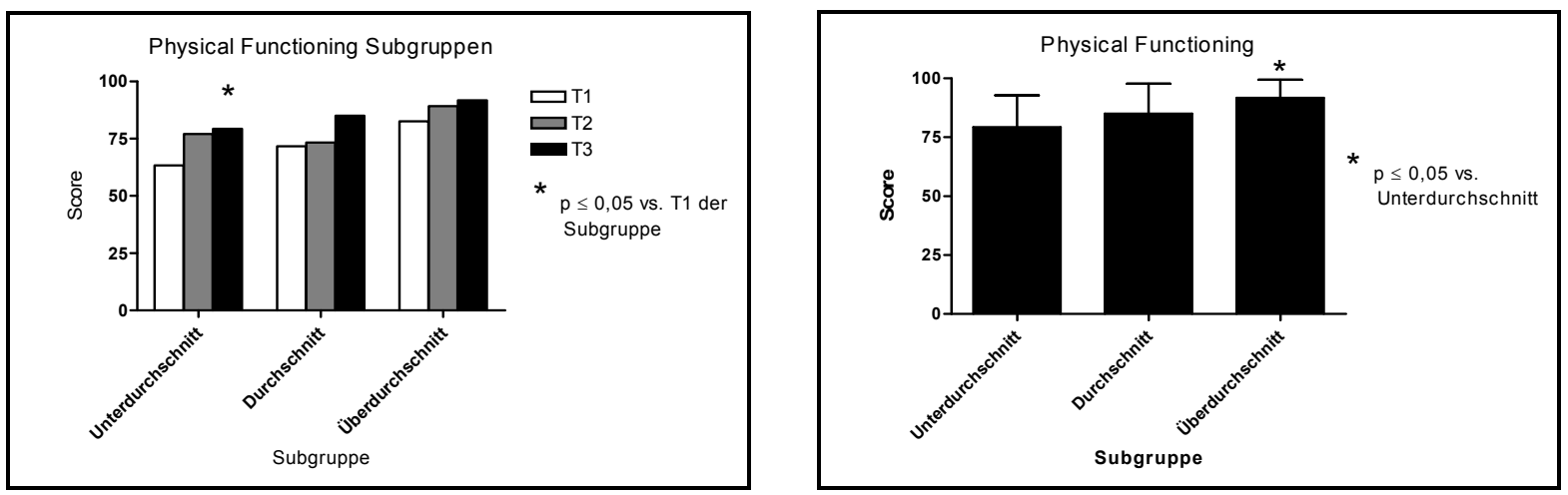

Abbildung 83: Physical Functioning zu allen Messzeitpunkten (links) und Physical Functioning zu T3 (rechts) in Abhängigkeit vom tatsächlichen Trainingsumfang

\begin{tabular}{|l|r|r|r|}
\hline & Unterdurchschnitt & Durchschnitt & Überdurchschnitt \\
\hline T1 & $63,33 \pm 16,7$ & $71,67 \pm 22,8$ & $82,50 \pm 11,8$ \\
\hline T2 & $77,05 \pm 14,6$ & $73,33 \pm 18,1$ & $89,17 \pm 7,9$ \\
\hline T3 & $79,33 \pm 13,5$ & $85,00 \pm 12,7$ & $91,67 \pm 7,8$ \\
\hline
\end{tabular}

Tabelle 59: Physical Functioning in Abhängigkeit vom tatsächlichen Trainingsumfang 
Die Subskala Emotional Functioning, dargestellt in Abbildung 84 (links), zeigte nur in der Subgruppe Unterdurchschnitt signifikante Unterschiede zwischen den Messzeitpunkten. Der Ausgangswert zu T1 war erneut in der Subgruppe Unterdurchschnitt niedriger als in den anderen Subgruppen.

Zu T3 konnten keine signifikanten Unterschiede zwischen den Subgruppen nachgewiesen werden. Der Mittelwert der Subgruppe Unterdurchschnitt lag mit $58,33 \pm 23,9$ niedriger als die Werte der anderen beiden Gruppen. Diese waren mit $75,00 \pm 18,9$ und $75,00 \pm 23,6$ im Mittel identisch.
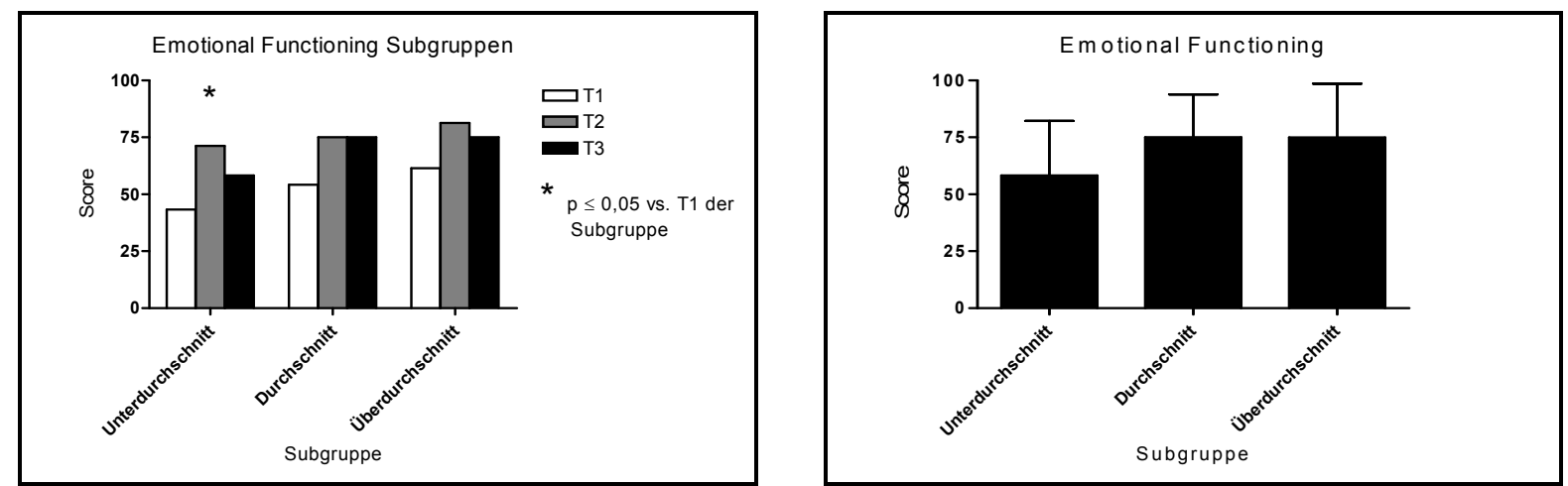

Abbildung 84: Emotional Functioning zu allen Messzeitpunkten (links) und Emotional Functioning zu T3 (rechts) in Abhängigkeit vom tatsächlichen Trainingsumfang

\begin{tabular}{|l|r|r|r|}
\hline & Unterdurchschnitt & Durchschnitt & Überdurchschnitt \\
\hline T1 & $43,33 \pm 17,9$ & $54,17 \pm 16,2$ & $61,46 \pm 29,9$ \\
\hline T2 & $71,30 \pm 16,2$ & $75,00 \pm 19,8$ & $81,25 \pm 19,3$ \\
\hline T3 & $58,33 \pm 23,9$ & $75,00 \pm 18,9$ & $75,00 \pm 23,6$ \\
\hline
\end{tabular}

Tabelle 60: Emotional Functioning in Abhängigkeit vom tatsächlichen Trainingsumfang

Die linke Seite der Abbildung 85 zeigt die Subskala Cognitive Functioning aller Subgruppen zu den drei Messzeitpunkten. Die Ausgangswerte zu T1 waren in der Subgruppe Unterdurchschnitt am niedrigsten. Allerdings konnten auch lediglich in dieser Subgruppe signifikante Veränderungen zwischen den Messzeitpunkten ermittelt werden.

Abbildung 85 (rechts) zeigt die Subskala Cognitive Functioning zu T3. Es konnten keine signifikanten Unterschiede zwischen den Subgruppen zu T3 nachgewiesen werden. 
Die Subgruppe Unterdurchschnitt lag mit einem Mittelwert von 70,00 $\pm 30,2$ unter dem

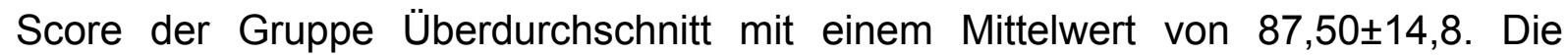
Subgruppe Durchschnitt hatte mit 62,50 $\pm 33,0$ den niedrigsten Wert.
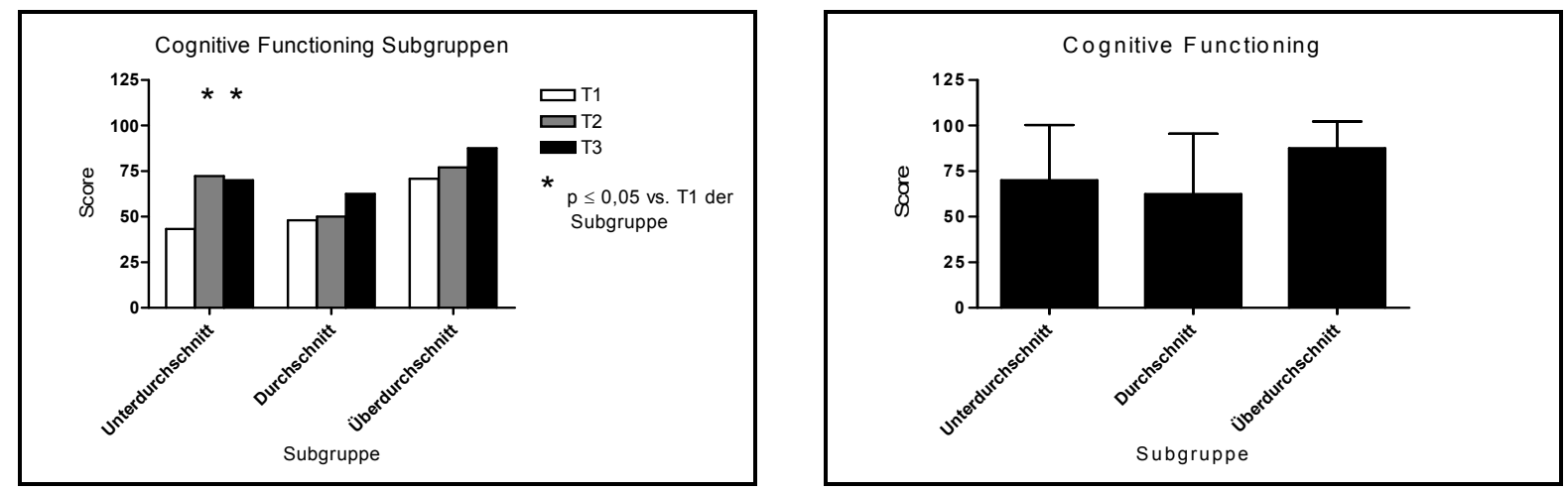

Abbildung 85: Cognitive Functioning zu allen Messzeitpunkten (links) und Cognitive Functioning zu T3 (rechts) in Abhängigkeit vom tatsächlichen Trainingsumfang

\begin{tabular}{|l|r|r|r|}
\hline & Unterdurchschnitt & Durchschnitt & Überdurchschnitt \\
\hline T1 & $43,33 \pm 26,3$ & $47,92 \pm 33,9$ & $70,83 \pm 30,2$ \\
\hline T2 & $72,22 \pm 22,1$ & $50,00 \pm 33,3$ & $77,08 \pm 33,0$ \\
\hline T3 & $70,00 \pm 30,2$ & $62,50 \pm 33,0$ & $87,50 \pm 14,8$ \\
\hline
\end{tabular}

Tabelle 61: Emotional Functioning in Abhängigkeit vom tatsächlichen Trainingsumfang 
Abbildung 86 (links) zeigt die Subskala Global Health aller drei Subgruppen zu den Messzeitpunkten. Die T1 Ausgangswerte der Subgruppen Unterdurchschnitt und Durchschnitt unterschieden sich nur gering. Der Ausgangswert der Subgruppe Überdurchschnitt lag erneut höher. Zudem ließen sich nur in dieser Gruppe signifikante Veränderungen zwischen den Messzeitpunkten nachweisen.

Zu T3 konnte mit einem Signifikanzwert von $p=0,0071$ ein signifikanter Unterschied

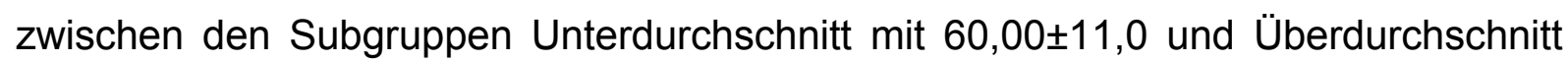
mit $79,17 \pm 15,4$ nachgewiesen werden. Die Subgruppe Durchschnitt lag mit einem Mittelwert von $68,75 \pm 20,3$ dazwischen.
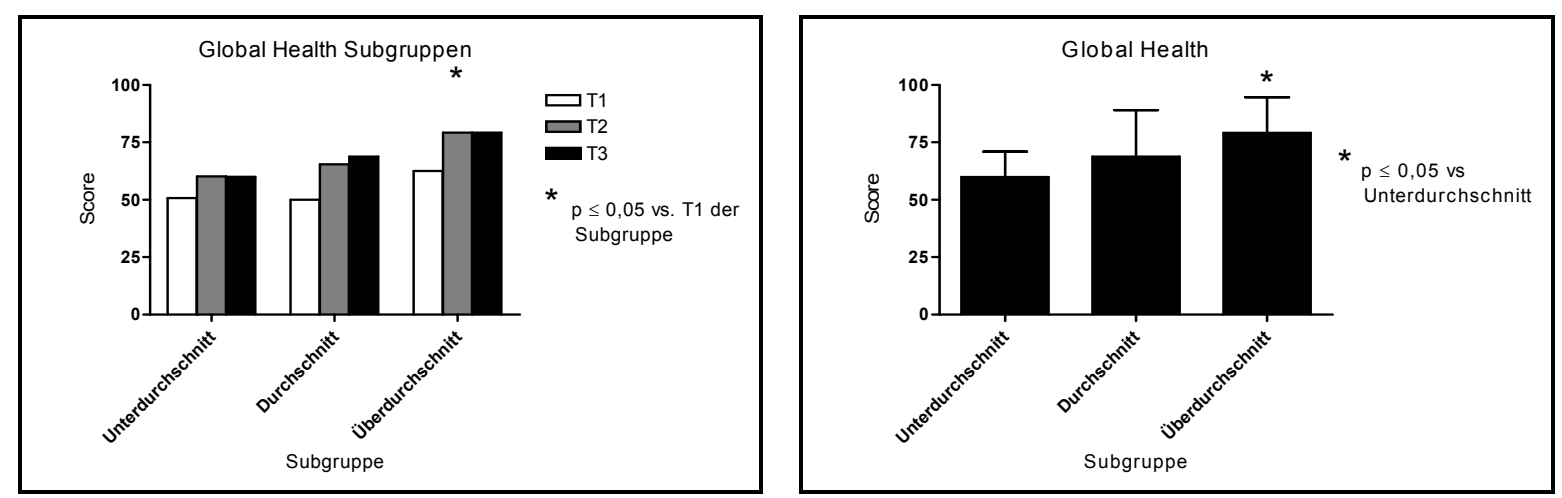

Abbildung 86: Global Health zu allen Messzeitpunkten (links) und Global Health zu T3 (rechts) in Abhängigkeit vom tatsächlichen Trainingsumfang

\begin{tabular}{|l|r|r|r|}
\hline & Unterdurchschnitt & Durchschnitt & Überdurchschnitt \\
\hline T1 & $50,83 \pm 13,9$ & $50,00 \pm 20,4$ & $62,50 \pm 18,4$ \\
\hline T2 & $60,19 \pm 16,6$ & $65,48 \pm 27,0$ & $79,17 \pm 10,9$ \\
\hline T3 & $60,00 \pm 11,0$ & $68,75 \pm 20,3$ & $79,17 \pm 15,4$ \\
\hline
\end{tabular}

Tabelle 62: Global Health in Abhängigkeit vom tatsächlichen Trainingsumfang

Abbildung 87 (links) zeigt die Subskala Fatigue aller Subgruppen zu den drei Messzeitpunkten. In den Subgruppen Unterdurchschnitt und Überdurchschnitt konnten signifikante Veränderungen zwischen den Messzeitpunkten nachgewiesen werden. In der Subgruppe Durchschnitt konnten keine Signifikanzen zwischen den Messzeitpunkten ermittelt werden. Der höchste Ausgangswert zu T1 wurde erneut in der Subgruppe Unterdurchschnitt ermittelt. 
Abbildung 87 (rechts) zeigt die Mittelwerte der Subgruppen zu T3. Mit einem Signifikanzwert von $\mathrm{p}=0,0270$ konnte ein signifikanter Unterschied zwischen der Subgruppe Unterdurchschnitt mit 45,56 $\pm 23,1$ und der Subgruppe Überdurchschnitt mit 22,20 $\pm 15,7$ nachgewiesen werden. Die Subgruppe Durchschnitt lag bei einem Mittelwert von $31,94 \pm 22,6$.
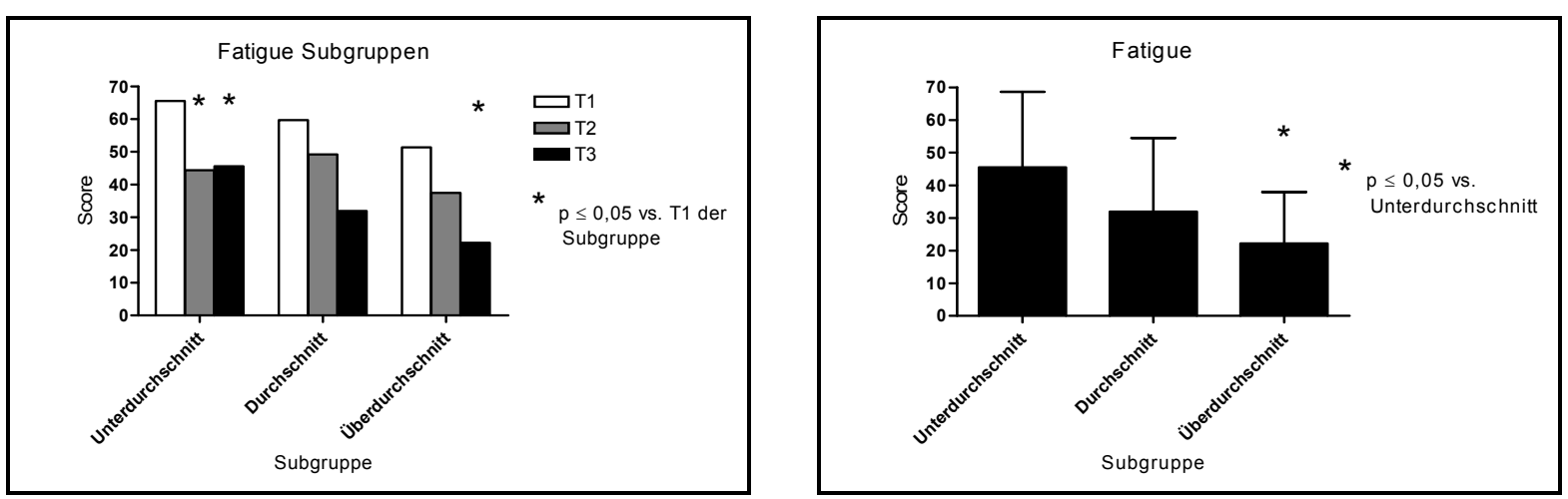

Abbildung 87: Fatigue zu allen Messzeitpunkten (links) und Fatigue zu T3 (rechts) in Abhängigkeit vom tatsächlichen Trainingsumfang

\begin{tabular}{|l|r|r|r|}
\hline & Unterdurchschnitt & Durchschnitt & Überdurchschnitt \\
\hline T1 & $65,56 \pm 18,5$ & $59,72 \pm 29,7$ & $51,39 \pm 24,4$ \\
\hline T2 & $44,44 \pm 18,4$ & $49,21 \pm 32,6$ & $37,50 \pm 10,2$ \\
\hline T3 & $45,56 \pm 23,1$ & $31,94 \pm 22,6$ & $22,20 \pm 15,7$ \\
\hline
\end{tabular}

Tabelle 63: Fatigue in Abhängigkeit vom tatsächlichen Trainingsumfang 
In Abbildung 88 (links) ist die Symptomsubskala Schmerz aller Subgruppen zu den drei Messzeitpunkten dargestellt. Es wird deutlich, dass sich die Ausgangswerte in den Subgruppen Unterdurchschnitt und Überdurchschnitt nur geringfügig unterschieden. Der T1 Wert der Subgruppe Durchschnitt war höher als bei den anderen Gruppen. Signifikante Veränderungen zwischen den Messzeitpunkten konnten in keiner Subgruppe nachgewiesen werden.

Abbildung 88 (rechts) zeigt, dass zu T3 keine signifikanten Unterschiede zwischen den Subgruppen nachweisbar waren. Die Tendenz der Mittelwerte zeigte allerdings das erwartete Bild. Die Subgruppe Unterdurchschnitt lag mit einem Mittelwert von $33,33 \pm 26,1$ über der Subgruppe Durchschnitt mit 29,17 $\pm 35,6$. Mit einem Mittelwert von $14,58 \pm 24,3$ lag die Subgruppe Überdurchschnitt darunter.
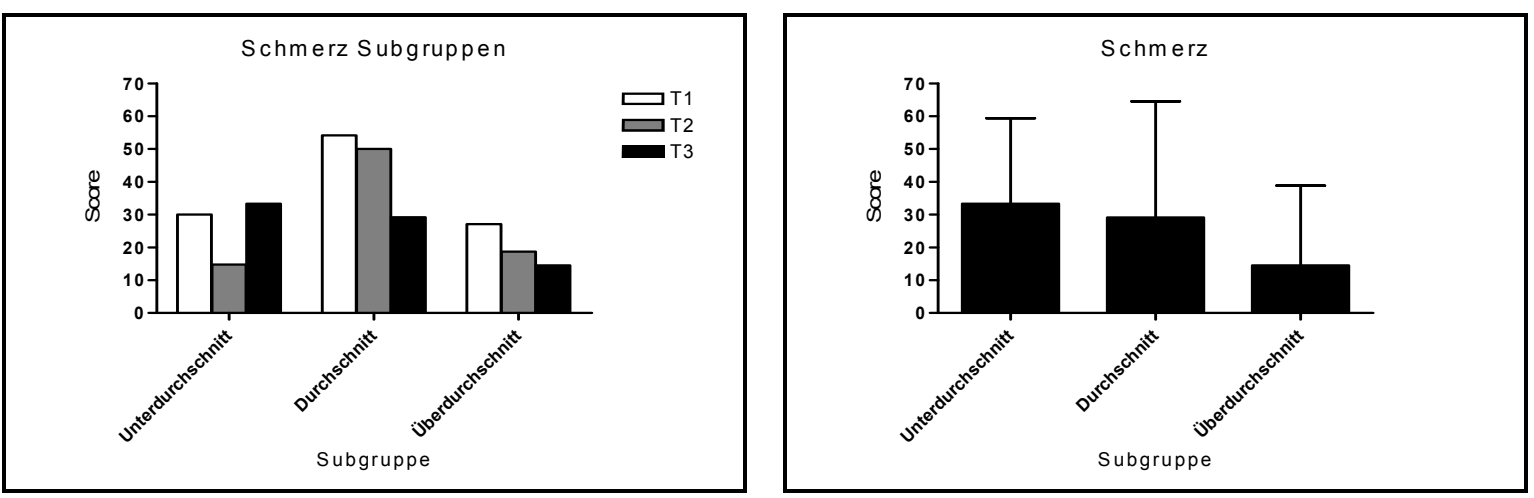

Abbildung 88: Schmerz zu allen Messzeitpunkten (links) und Schmerz zu T3 (rechts) in Abhängigkeit vom tatsächlichen Trainingsumfang

\begin{tabular}{|l|r|r|r|}
\hline & Unterdurchschnitt & Durchschnitt & Überdurchschnitt \\
\hline T1 & $30,00 \pm 20,5$ & $54,17 \pm 38,6$ & $27,08 \pm 33,3$ \\
\hline T2 & $14,81 \pm 15,5$ & $50,00 \pm 41,9$ & $18,75 \pm 30,1$ \\
\hline T3 & $33,33 \pm 26,1$ & $29,17 \pm 35,6$ & $14,58 \pm 24,3$ \\
\hline
\end{tabular}

Tabelle 64: Schmerz in Abhängigkeit vom tatsächlichen Trainingsumfang

Abbildung 89 (links) zeigt die Symptomsubskala Schlaflosigkeit für alle drei Subgruppen zu den Messzeitpunkten T1, T2 und T3. Ein hoher Score in dieser Skala lässt erneut auf eine starke Ausprägung des Symptoms schließen. Die Ausgangswerte der Subgruppen Durchschnitt und Überdurchschnitt sind niedriger als in der Subgruppe Unterdurchschnitt. Allerdings konnten nur in der Subgruppe 
Unterdurchschnitt signifikante Unterschiede zwischen den Messzeitpunkten nachgewiesen werden.

Abbildung 89 (rechts) stellt die Mittelwerte der Subgruppen zu T3 dar. Es zeigt sich ein ähnliches Bild wie bei der LASA-Skala Schlafqualität des Fatiguerhythmikbogens. Mit einem Mittelwert von 45,83 $\pm 29,6$ lag die Subgruppe Durchschnitt über den Werten der anderen beiden Subgruppen. Deren Mittelwerte lagen bei 30,00 $\pm 29,2$ bei der Gruppe Unterdurchschnitt und 25.00\$34,5 bei der Gruppe Überdurchschnitt.
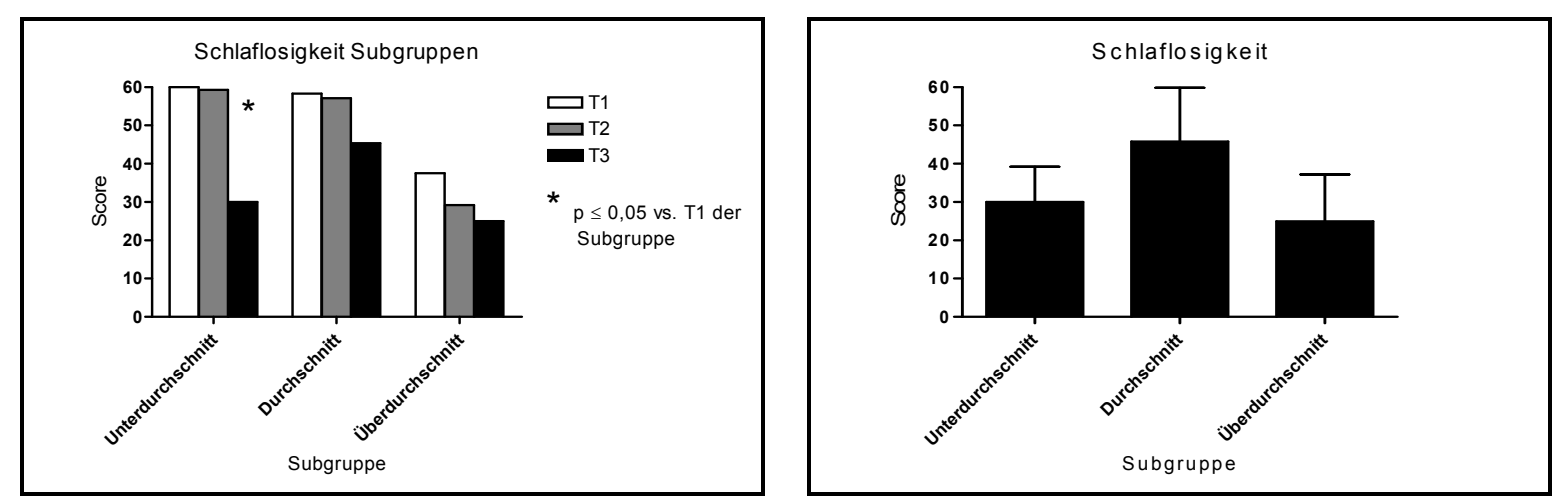

Abbildung 89: Schlaflosigkeit zu allen Messzeitpunkten (links) und Schlaflosigkeit zu T3 (rechts) in Abhängigkeit vom tatsächlichen Trainingsumfang

\begin{tabular}{|l|r|r|r|}
\hline & Unterdurchschnitt & Durchschnitt & Überdurchschnitt \\
\hline T1 & $70,00 \pm 36,7$ & $58,33 \pm 34,5$ & $37,50 \pm 37,5$ \\
\hline T2 & $59,26 \pm 27,8$ & $57,14 \pm 31,7$ & $29,19 \pm 37,5$ \\
\hline T3 & $30,00 \pm 29,2$ & $45,83 \pm 39,6$ & $25,00 \pm 34,5$ \\
\hline
\end{tabular}

Tabelle 65: Schlaflosigkeit in Abhängigkeit vom tatsächlichen Trainingsumfang 


\subsubsection{Auswirkungen des tatsächlichen Trainingsumfangs auf verschiedene Subskalen des FACIT-F}

Abbildung 90 (links) zeigt die Ergebnisse der Subskala Körperliches Wohlbefinden der drei Subgruppen zu allen drei Messzeitpunkten. Die Ausgangswerte zu T1 unterschieden sich in den Subgruppen nur gering. Signifikante Veränderungen zwischen den Messzeitpunkten konnten nur in der Subgruppe Überdurchschnitt nachgewiesen werden.

Abbildung 90 (rechts) zeigt die Mittelwerte der Subgruppen zu T3. Es wird deutlich, dass der Score im Mittel von der Subgruppe Unterdurchschnitt mit einem Mittelwert

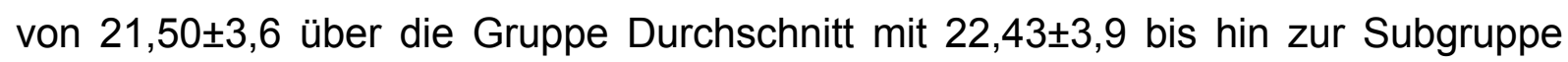
Überdurchschnitt mit 25,14 $\pm 3,1$ anstieg. Mit einem Signifikanzwert von $p=0,0461$ war der Score der Gruppe Überdurchschnitt signifikant höher gegenüber dem der Gruppe Unterdurchschnitt.
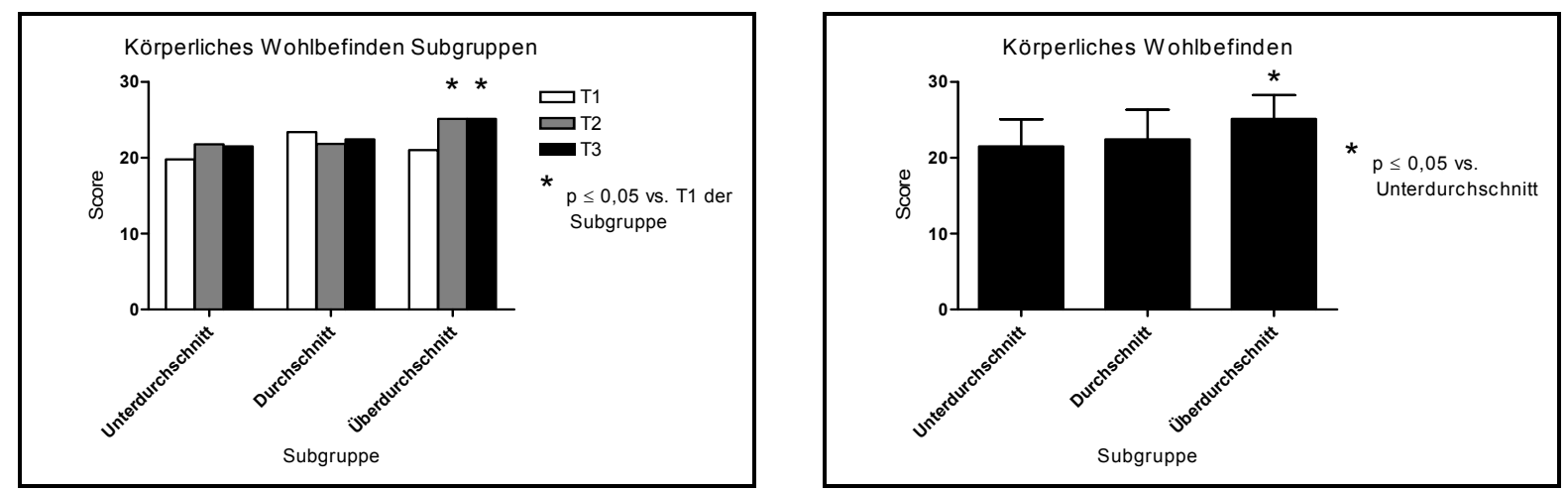

Abbildung 90: Körperliches Wohlbefinden zu allen Messzeitpunkten (links) und Körperliches Wohlbefinden zu T3 (rechts) in Abhängigkeit vom tatsächlichen Trainingsumfang

\begin{tabular}{|l|r|r|r|}
\hline & Unterdurchschnitt & Durchschnitt & Überdurchschnitt \\
\hline T1 & $19,80 \pm 4,2$ & $23,38 \pm 3,7$ & $21,00 \pm 3,7$ \\
\hline T2 & $21,83 \pm 6,0$ & $21,83 \pm 6,0$ & $25,13 \pm 2,4$ \\
\hline T3 & $21,50 \pm 3,6$ & $22,43 \pm 3,9$ & $25,14 \pm 3,1$ \\
\hline
\end{tabular}

Tabelle 66: Körperliches Wohlbefinden in Abhängigkeit vom tatsächlichen Trainingsumfang 
Auf der linken Seite der Abbildung 91 sind die Ergebnisse aller Subgruppen der Subskala Funktionsfähigkeit zu den drei Messzeitpunkten dargestellt. Der Ausgangswert der Subgruppe Unterdurchschnitt war hierbei am niedrigsten. Signifikante Unterschiede zwischen den Messzeitpunkten konnten in keiner Subgruppe ermittelt werden.

Abbildung 91 (rechts) zeigt die Mittelwerte der Funktionsfähigkeit der drei Subgruppen zu T3. In der Subgruppe Unterdurchschnitt wurde ein Mittelwert von $18,90 \pm 3,8$ gemessen. Bei der Subgruppe Durchschnitt lag der Mittelwert niedriger bei $17,29 \pm 5,1$ und bei der Subgruppe Überdurchschnitt lag dieser bei $23,00 \pm 3,5$. Mit einem Signifikanzwert von $p=0,0407$ war die Subgruppe Unterdurchschnitt signifikant gegenüber der Subgruppe Überdurchschnitt. Die Subgruppe Durchschnitt war mit einem Signifikanzwert von $p=0,0303$ ebenfalls signifikant gegenüber der Subgruppe Überdurchschnitt.
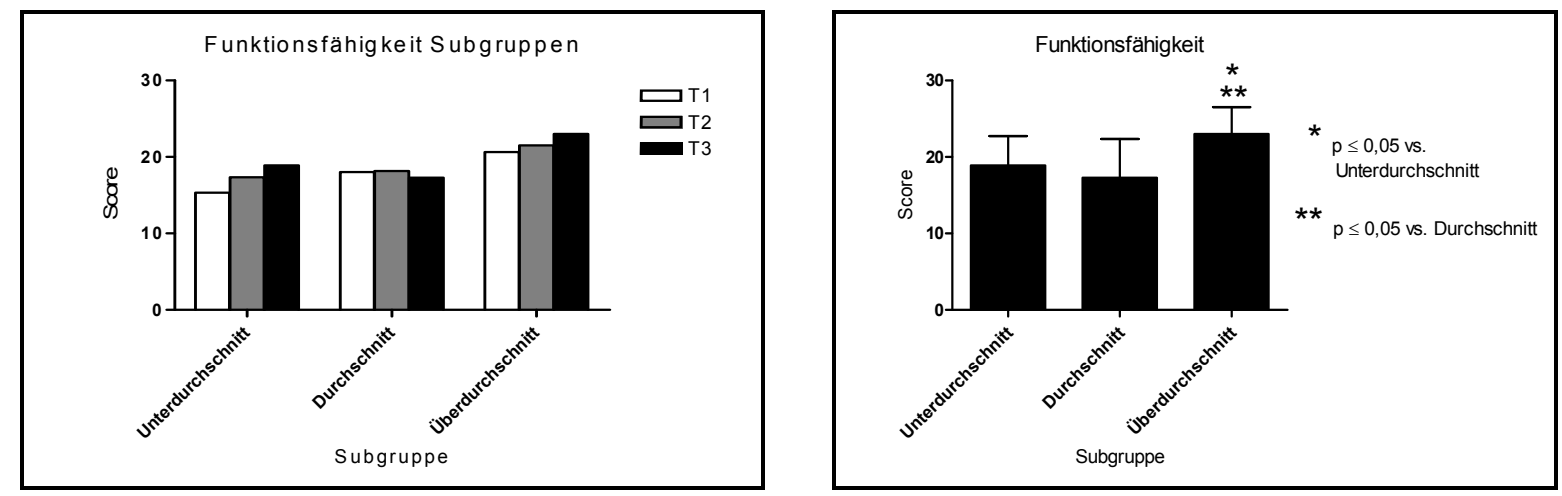

Abbildung 91: Funktionsfähigkeit zu allen Messzeitpunkten (links) und Funktionsfähigkeit zu T3 (rechts) in Abhängigkeit vom tatsächlichen Trainingsumfang

\begin{tabular}{|l|r|r|r|}
\hline & Unterdurchschnitt & Durchschnitt & Überdurchschnitt \\
\hline T1 & $15,30 \pm 4,8$ & $19,00 \pm 3,5$ & $20,63 \pm 2,7$ \\
\hline T2 & $17,33 \pm 4,6$ & $18,17 \pm 5,3$ & $21,50 \pm 5,2$ \\
\hline T3 & $18,90 \pm 3,8$ & $17,29 \pm 5,1$ & $23,00 \pm 3,5$ \\
\hline
\end{tabular}

Tabelle 67: Funktionsfähigkeit in Abhängigkeit vom tatsächlichen Trainingsumfang 
Abbildung 92 (links) zeigt die Ergebnisse der Subskala Seelisches Wohlbefinden aller Subgruppen zu den drei Messzeitpunkten. Die Ausgangswerte der Subgruppen zu T1 waren annährend identisch. Signifikante Unterschiede zwischen den Messzeitpunkten konnten in keiner Subgruppe nachgewiesen werden.

In Abbildung 92 (rechts) sind die Mittelwerte der Subgruppen zu T3 dargestellt. Es konnten keine signifikanten Unterschiede zwischen den drei Subgruppen nachgewiesen werden. Die Subgruppe Unterdurchschnitt hatte einen Mittelwert von $19,70 \pm 3,1$. In der Subgruppe Durchschnitt lag dieser bei $18,57 \pm 2,6$ und in der Gruppe Überdurchschnitt bei 20,71 $\pm 3,4$.
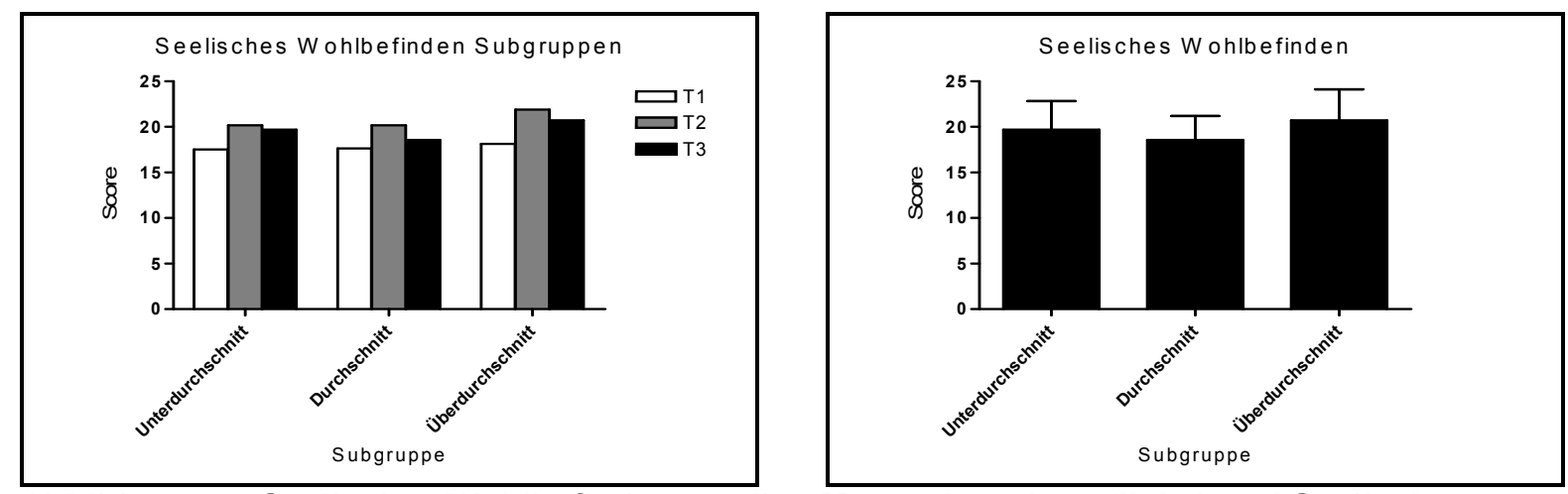

Abbildung 92: Seelisches Wohlbefinden zu allen Messzeitpunkten (links) und Seelisches Wohlbefinden zu T3 (rechts) in Abhängigkeit vom tatsächlichen Trainingsumfang

\begin{tabular}{|l|r|r|r|}
\hline & Unterdurchschnitt & Durchschnitt & Überdurchschnitt \\
\hline T1 & $17,50 \pm 5,1$ & $17,63 \pm 6,0$ & $18,13 \pm 3,6$ \\
\hline T2 & $20,17 \pm 3,0$ & $20,17 \pm 3,0$ & $21,88 \pm 2,2$ \\
\hline T3 & $19,70 \pm 3,1$ & $18,57 \pm 2,6$ & $20,71 \pm 3,4$ \\
\hline
\end{tabular}

Tabelle 68: Seelisches Wohlbefinden in Abhängigkeit vom tatsächlichen Trainingsumfang

Abbildung 93 zeigt die Ergebnisse des Fatiguemoduls des FACIT-F. Auf der linken Seite der Abbildung sind die Mittelwerte der Subgruppen zu allen drei Messzeitpunkten dargestellt. Signifikante Unterschiede zwischen den Messzeitpunkten ließen sich in den Subgruppen Unterdurchschnitt und Überdurchschnitt nachweisen. Die Ausgangswerte der Subgruppen zu T1 unterschieden sich nur geringfügig. 
Es ließen sich in dieser Subskala zum Messzeitpunkt T3 keine signifikanten Unterschiede zwischen den einzelnen Subgruppen nachweisen. Die Subgruppe

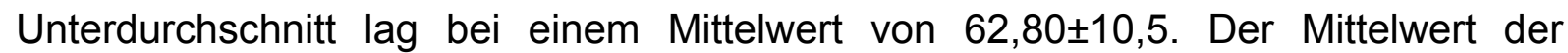
Subgruppe Durchschnitt lag bei $61,57 \pm 9,6$ und in der Subgruppe Überdurchschnitt bei $54,86 \pm 7,3$
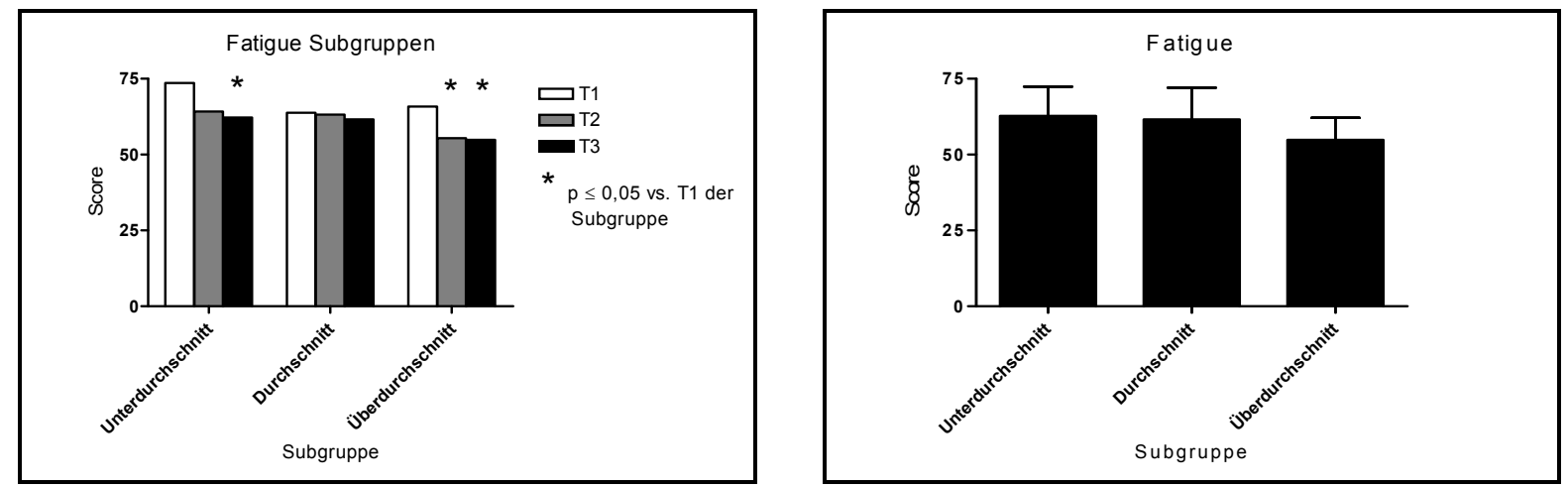

Abbildung 93: Fatigue zu allen Messzeitpunkten (links) und Fatigue zu T3 (rechts) in Abhängigkeit vom tatsächlichen Trainingsumfang

\begin{tabular}{|l|r|r|r|}
\hline & Unterdurchschnitt & Durchschnitt & Überdurchschnitt \\
\hline T1 & $73,60 \pm 10,0$ & $63,75 \pm 10,5$ & $65,86 \pm 9,3$ \\
\hline T2 & $64,17 \pm 15,5$ & $63,17 \pm 14,5$ & $55,38 \pm 5,4$ \\
\hline T3 & $62,80 \pm 9,5$ & $61,57 \pm 10,5$ & $54,86 \pm 7,3$ \\
\hline
\end{tabular}

Tabelle 69: Fatigue in Abhängigkeit vom tatsächlichen Trainingsumfang 


\section{Diskussion}

Ziel der vorliegenden Studie war die Entwicklung eines funktionellen rehabilitativen Krafttrainings zur Behandlung tumorbedingter Fatigue. Hierbei sollte insbesondere untersucht werden, wie sich regelmäßiges Training auf die zirkadiane Rhythmik der Fatigue auswirkt. Zusätzlich wurde die Wirksamkeit bezüglich zahlreicher Einflussgrößen der Lebensqualität und der körperlichen Leistungsfähigkeit untersucht. Die gewonnenen Ergebnisse der Studie werden in diesem Kapitel diskutiert und in den aktuellen Forschungsstand eingebettet.

Zusätzlich wird die Patientenrekrutierung und die Methodik der Datenerhebung diskutiert. Im Fokus stehen der gesamte Studienablauf sowie die verwendeten Messinstrumente.

Während der gesamten Diskussion soll ständig ein Bezug zu den im Vorfeld aufgestellten Hypothesen (Abschnitt 5.1) aufrecht erhalten werden, so dass diese überprüft werden können.

\subsection{Diskussion Material und Methoden}

\subsubsection{Probandenkollektiv}

Die Patientenrekrutierung für die Studie dauerte über ein Jahr. Dabei füllten 1089 Patienten den Fatigue-LASA Screeningbogen bei Ankunft in der Klinik aus. Von diesen Patienten erfüllten $792(72,7 \%)$ das Kriterium einen LASA Score $\geq 4 \mathrm{zu}$ haben und somit die Informationsveranstaltung besuchen zu können.

101 Patienten gaben ihre Einverständniserklärung ab, passten ins Studiendesign und absolvierten schließlich den Messtermin T1. Von diesen nahmen noch 78 (77,2 \%) am Messtermin T2 teil. Den Messzeitpunkt T3, und somit die gesamte Studie, absolvierten noch 66 (65,3\%) Patienten. Insgesamt handelte es sich um einen Drop Out von 35 (34,7 \%) Patienten.

Die Drop Out Quote von 34,7 \% war relativ hoch, was in Studien mit Tumorpatienten jedoch nicht unnormal ist. Eine Begründung ist vor allem damit gegeben, dass die 
Teilnahme an einer Studie mit einem erhöhten Aufwand und Belastung verbunden ist. Da sich die Patienten mit ihrer Krankheit und deren Therapie oftmals voll ausgelastet fühlten, wollten sie sich mit der Teilnahme an einer Studie nicht noch zusätzlich belasten. Zusätzlich gilt es zu bedenken, dass die Probanden ohnehin schon einen Fatiguescore $\geq 4$ angaben. Eine zusätzliche Belastung könnte zu einer Überforderung führen. Oftmals hatten die Patienten Angst davor sich zu überlasten.

Wiederholt wirkte das zwei- bis dreimal wöchentliche Trainingsprogramm abschreckend für Patienten, die nur wenig sportliche Vorerfahrungen hatten und sich nicht vorstellen konnten ein Heimtrainingsprogramm in ihren Altag zu integrieren. Andererseits gab es während der Rekrutierungsphasen der Kontrollgruppe immer wieder Patienten, die gern an der Studie teilgenommen hätten, wenn sie in die Trainingsgruppe gekommen wären.

Auffällig war, dass der Drop Out zwischen T2 und T3 deutlich geringer ausfiel als vorher. Lediglich 12 (11,8 \%) Patienten zogen in diesem Zeitraum ihre Einwilligung zurück.

Die Motivation zur Teilnahme war bei Patienten zwischen 41 - 60 Jahren am höchsten. Hier lag der Anteil der Probanden bei $74,7 \%$. Allerdings gehörte der Hauptanteil der Patienten der Klinik dieser Altersgruppe an. Im Alter von 61 - 75 Jahren waren immerhin noch ca. 19,8\% der Probanden.

Zudem waren $88 \%$ der Probanden weiblich und nur 12\% männlich. Ursache dafür war der deutlich geringere Anteil männlicher Patienten in der Klinik, aber auch die Tatsache, dass sich nur wenige Männer zur Teilnahme an der Studie bereit erklärten. Außerdem war der Anteil der Brustkrebspatientinnen im Probandenkollektiv außerordentlich hoch, was eine weitere Erklärung des hohen Frauenanteils war. Aufgrund dieses Missverhältnisses wurden die Ergebnisse nicht geschlechtsspezifisch ausgewertet.

Der Anteil der sportlich Aktiven war in der Gruppe der Probanden hoch. Lediglich 15,6\% der Probanden gaben an, vor der Erkrankung überhaupt keinen Sport gemacht zu haben. Vor der Rehabilitation betrieb bereits ein Anteil von 28,9\% keinen Sport mehr. Die restlichen Probanden gaben an, regelmäßig oder zumindest unregelmäßig sportlich aktiv zu sein. 
Es fällt auf, dass der Anteil der sportlich Aktiven und damit auch zum Sport motivierten Probanden sehr hoch war. Da es sich um eine Studie handelte, die einen sportwissenschaftlichen Hintergrund hatte, ist diese Tatsache nicht weiter verwunderlich.

Natürlich muss in der Interpretation der Studie hinterfragt werden, in wie weit es sich tatsächlich um eine repräsentative Stichprobe handelt. Gewisse Selektionsprozesse lassen sich nicht komplett ausschließen. So kann beispielsweise angenommen werden, dass die Sonnenberg-Klinik aufgrund ihres sportorientierten Profils besonders von sportinteressierten Patienten zur Rehabilitation besucht wird. Ein anderer Punkt wäre, dass der Anteil an Frauen in der onkologischen Rehabilitation grundsätzlich sehr viel höher liegt als der Männeranteil. Verschiedene andere Selektionsprozesse könnten in die Diskussion mit einfließen. Bei der Unterschiedlichkeit des Probandenkollektivs ließen sich tatsächliche störende Einflussfaktoren allerdings nicht feststellen und wurden somit vernachlässigt.

\subsubsection{Messinstrumente}

\section{Fragebögen}

Wie in Abschnitt 5.6 bereits erläutert kamen zur Messung Fragebögen und leistungsdiagnostische Verfahren zum Einsatz.

Bei den verwendeten Fragebögen HADS, MFI EORTC QLQ-C30 und FACIT-F handelt es sich um standardisierte Messinstrumente, die von den jeweiligen Verfassern (Abschnitt 5.6.1) auf die Hauptgütekriterien hin geprüft wurden und aufgrund ihrer Genauigkeit und einfachen Handhabung in zahlreichen Studien zur Anwendung kamen (MINTON \& STONE, 2009).

Ausnahme bildeten der Dokumentationsbogen und der Fatiguerhythmikbogen. Der Dokumentationsbogen diente einzig zur Abfragung soziodemographischer, anthropometrischer und sportbezogener Daten und war daher in seiner Handhabung unkompliziert.

Bei dem Fatiguerhythmikbogen handelte es sich um eine abgewandelte Fatigue LASA-Skala, die zu mehreren Tageszeiten abgefragt wurde. LASA-Skalen wurden bereits in zahlreichen klinischen Studien verwendet und auf Validität und Reliabilität 
überprüft. Speziell im Bereich der Lebensqualitätsforschung bei Tumorpatienten wurden LASA-Skalen mit verschiedensten standardisierten Fragebögen korreliert, was hohe Korrelationen ergab (LOCKE et al. 2007).

Der Fatigue-LASA Screeningbogen zum Anfang der Untersuchung hatte außerdem den Vorteil, die Probandenrekrutierung stark zu vereinfachen. Mit Hilfe der Skala konnte gewährleistet werden, dass nur betroffene Patienten die Aufklärungsveranstaltung besuchten. Zudem konnte mit Hilfe der Daten eine Vollerhebung aller im Messzeitraum behandelten Patienten durchgeführt werden. Als Problem stellte sich dabei heraus, dass zwar sichergestellt werden konnte, dass jeder Patient der Klinik einen LASA-Bogen bekam, allerdings konnte keine genaue Rücklaufquote ermittelt werden.

Mit Ausnahme einiger Schwierigkeiten bei der fristgerechten Abgabe der Fragebögen verliefen die Befragungen zu allen drei Messzeitpunkten weitestgehend unproblematisch.

\section{Leistungsdiagnostik}

Der 6-Minuten Gehtest ist ein sehr ökonomischer Test. Mit einem geringen apparativen Aufwand ist ein aussagekräftiges Ergebnis möglich. Wie schon in Abschnitt 5.6.2.2 beschrieben, ist der Gehtest ein nicht unübliches Messverfahren um Ausdauerleistungen ohne großen apparativen Aufwand zu überprüfen. Durch die in einigen Studien ermittelte Korrelation zwischen 6-Minuten Gehtest und $\mathrm{VO}_{2 \max }$ Messung kann davon ausgegangen werden, dass der 6-Minuten Gehtest verlässliche Ergebnisse im Bezug auf die aerobe Ausdauerleistung liefert (OPASICH et al. 2001; ZUGCK et al. 2000; LUCAS et al. 1999; FAGGIANO et al. 1997; CAHALIN et al. 1996; RILEY et al. 1992). Bereits 1986 wurden die Variationen der Gehstrecke bei unterschiedlichem $\mathrm{VO}_{2 m a x}$ Wert untersucht und festgestellt, dass bei Patienten mit hohem VO2max die Gehstrecke deutlich weniger variiert (LIPKIN et al. 1986).

Der PWC 130 lieferte in dieser Studie nur unzureichende Ergebnisse. Viele Patienten hatten Schwierigkeiten überhaupt einen Zielpuls von 130 Schlägen/Minute zu erreichen. In einigen Fällen waren die Patienten bereits zu erschöpft bevor sie den Zielpuls erreichten. Andere hatten nicht die Muskelkraft, um eine Wattzahl zu treten, 
die sie bis an den Zielpuls heranführt. Zum Teil konnten die Patienten auch aufgrund ihres Alters nicht bis zu diesem Zielpuls belastet werden. In diesen Fällen musste der Test vorzeitig abgebrochen werden, um eine Gefährdung der Patienten auszuschließen.

Die Kraftmessung mit Hilfe des digitalen Messgerätes der Firma DIGGIMAX stellte sich als sinnvolle und problemlos durchführbare Methode zur Messung der isometrischen Maximalkraft heraus. In Messaufbau und Durchführung konnte, abgesehen von geringen Abweichungen, eine standardisierte Messposition vorgegeben werden. Die Messposition wurde optimal an die Körpergröße und Statur angeglichen. Messhaltung und Gelenkwinkel der Probanden wurden vor der Messung sorgfältig ausgerichtet, so dass die Störvariable der unterschiedlichen Messposition minimiert wurde. Trotzdem konnten geringe Unterschiede in der Messposition, unterschiedliche Ausführung und unterschiedliche Motivation zur maximalen Belastung als Störfaktoren bei der Kraftmessung nicht vollständig ausgeschlossen werden. Durch die Angst beschädigte Strukturen maximal zu belasten, war die Motivation zur maximalen Belastung oftmals ein wesentlicher Störfaktor. Durch Probedurchläufe und Gewöhnung an das Gerät wurde versucht diesen Faktor weitestgehend zu minimieren.

Die Datenspeicherung und Auswertung erfolgte direkt durch die Messsoftware des Herstellers. Mit Hilfe einer direkten Diagrammerstellung konnten die Messresultate direkt im Anschluss für die Patienten verbildlicht dargestellt werden.

Als Diskussionspunkt muss angemerkt werden, dass es aus organisatorischen Gründen nicht möglich war die Probanden immer zur gleichen Tageszeit zu testen. Unterschiedliche Tageszeiten, Vorbelastungen und andere Parameter beeinflussten somit die Ergebnisse, was jedoch unvermeidbar war.

\subsubsection{Diskussion des Übungsprogramms}

Das Übungsprogramm beinhaltete acht funktionelle Kraftübungen. Es handelte sich um ein funktionelles dynamisches Kraftausdauertraining, welches speziell an die Bedürfnisse des durchschnittlichen Tumorpatienten angepasst wurde. Die 
Ergebnisse der Trainingsgruppe, die das Übungsprogramm durchführte, waren in nahezu allen untersuchten Bereichen der Lebensqualität, der Fatigue und der Leistungsdiagnostik verbessert, was bei der Kontrollgruppe nur zum Teil der Fall war. Spence et al. geben einen Überblick über verschiedenste Studien, die belegen, dass sich Übungsprogramme mit Inhalten des Krafttrainings während der Rehabilitationsphase onkologischer Patienten durchweg positiv auswirken (SPENCE et al. 2009). Auch Baumann et al. konnten in Studien zeigen, dass sich Übungsprogramme bei verschiedenen Krebsarten und in verschiedenen Phasen der Therapierung positiv auf physische Leistungsfaktoren, Fatigue und Lebensqualität auswirken (BAUMANN et al. 2010; BAUMANN \& FAUSER 2010).

In vielen Fällen bringt die häusliche Durchführung von selbstständigem Training auch Nachteile mit sich. Das Fehlen einer Bewegungskontrolle kann speziell bei Patienten mit geringem Körpergefühl zu falschen Bewegungsausführungen führen. Erfahrungsgemäß reicht ein schriftliches Handout zu den Übungen nicht aus, um eine fehlerfreie Ausführung zu gewährleisten.

Durch eine längere Einweisungsphase und eine ständige Überprüfung und Korrektur der Übungsausführung während der gesamten Zeit der stationären Rehabilitation sollte falschen Bewegungsmustern vorgebeugt werden. Tatsächlich gab es am Ende der stationären Rehabilitation nur noch wenige Patienten, die Schwierigkeiten mit der Ausführung einzelner Übungen hatten.

Zusätzlich spielt der motivationale Aspekt eines selbstständigen Trainings im häuslichen Umfeld eine nicht zu unterschätzende Rolle. Vielen Patienten fällt es schwer, sportliche Aktivität in ihren wöchentlichen Ablauf zu integrieren und eine Kontinuität zu gewährleisten. Mit 90,9\% des optimalen Trainingsumfangs war die Kontinuität und Regelmäßigkeit des Trainings aber durchaus hoch einzuschätzen.

Trotzdem konnte in den prozentualen Werten des optimalen Trainingsumfanges eine erhebliche Schwankungsbreite zwischen den Übungen festgestellt werden (Abb. 68). Die absolute Durchführungshäufigkeit schwankte stark. Dies könnte mitunter aus den unterschiedlichen Schwierigkeitsgraden der einzelnen Übungen oder auch der Motivation zu der jeweiligen Übung resultieren. Diese ist oftmals nicht bei allen Übungen gleich hoch. Speziell Übungen mit hohem Schwierigkeitsgrad bedürfen auch einer hohen Motivation.

Zum Anderen wurde der Schwierigkeitsgrad der Übungen, der zu den Werten des optimalen Trainingsumfangs führte, im Vorfeld subjektiv eingeschätzt. Die Übungen 
hatten also je nach ermessenem Schwierigkeitsgrad unterschiedliche optimale Trainingsumfänge. Dies lässt sich leicht am Beispiel verdeutlichen. Mit $3 \times 15$ Wiederholungen wurde der Schwierigkeitsgrad für Übung 3 vielleicht etwas zu hoch, während dieser mit $3 \times 10$ bei Übung 7 etwas zu niedrig eingeschätzt wurde. Mit einer Regulation der vorgegebenen Wiederholungszahlen und somit dem optimalen Trainingsumfang könnten diese Schwankungen ausgeglichen werden.

\subsubsection{Diskussion des Dokumentationsbogens zum Training}

Die Dokumentation des Trainings in der Zeit zwischen T2 und T3 brachte zum Teil Probleme mit sich. In den meisten Fällen wurden die Dokumentationsbögen zum Training gewissenhaft und sehr detailliert ausgefüllt. In einigen Fällen erschienen auf dem Bogen hingegen unerklärliche Trainingspausen oder in einigen Fällen sogar der Trainingsabbruch. Zudem stellte sich aufgrund mangelnder Kontrollmöglichkeiten die Frage nach der Objektivität des Dokumentationsbogens. Wird davon ausgegangen, dass alle Angaben korrekt und wahrheitsgemäß dokumentiert wurden, handelte es sich insgesamt aber um ein sinnvolles Kontroll- und Messsystem.

\subsubsection{Diskussion der Subgruppen}

Die anschließende Auswertungsmethode der Berechnung des optimalen Trainingsumfangs hatte zum Einen den Zweck Trainingstreue und Motivation zu überprüfen und zum Anderen eine Verbindung zwischen dem Training und verschiedenen Parametern wie Lebensqualität, Fatigue, Angst und Depression herzustellen. Hierzu wurden Subgruppen gebildet. Die Grenzen zwischen den Subgruppen wurden eigenständig festgelegt. Die Auswertungsmethode als solches war intuitiv. Die Subgruppen hatten allerdings nur eine geringe Größe von 8 bis 11 Personen. Statistisch wäre es von Vorteil gewesen, größere Subgruppen zu bilden, was aufgrund der Gesamtanzahl von Probanden allerdings nicht möglich war. 


\subsection{Diskussion der Ergebnisse}

Im folgenden Abschnitt werden die Ergebnisse aus Kapitel 6 diskutiert. Von großer Wichtigkeit erscheint es, die gewonnen Erkenntnisse mit dem bisherigen Stand der Forschung zu vergleichen, die Ergebnisse entsprechend $\mathrm{zu}$ gewichten und einzuordnen. Auf die Beantwortung der Hypothesen soll besonders geachtet werden.

\subsubsection{Diskussion der Pre-Test Ergebnisse}

Der Pre-Test wurde über einen Zeitraum von vier Monaten durchgeführt, um vorläufige Erkenntnisse über die mögliche Probandengruppe zu gewinnen und die angestrebte Fragestellung auf ihre Durchführbarkeit zu überprüfen. Es konnte mit einer niedrigen Probandenzahl Durchführung und Schwierigkeiten der Datenerhebung unter vereinfachten Bedingungen getestet werden. Zusätzlich verlängerte sich durch den Pre-Test die Gesamtzeit der Vollerhebung des FatigueLASA, was einen zusätzlichen Anstieg der Teilnehmer zur Folge hat. Ferner konnte mit dem Pre-Test die Grundmenge der Probanden, bei der eine Tagesverlaufsdiagnostik der Fatigue durchgeführt wurde, erhöht werden. Die Ergebnisse des Pre-Tests unterschieden sich nur marginal von denen der eigentlichen Studie. Sie stellten daher nur eine Erweiterung der Datenmenge dar.

\subsubsection{Diskussion der Fatigue-LASA Ergebnisse}

Mit dem Fatigue-LASA wurde eine Vollerhebung während der gesamten Studienphase inklusive Pre-Test durchgeführt. Dabei wurden 1324 Fatigue-LASA Bögen ausgewertet, von denen 984 Patienten einen Fatigue Score $\geq 4$ angaben. Somit leiden $74,3 \%$ aller in diesem Zeitraum befragten Patienten zu Beginn ihres Rehabilitationsaufenthalts unter Fatigue. 403 Patienten, was 30,4\% des gesamten Untersuchungskollektivs ausmacht, geben einen Wert von 7-10 an und leiden somit unter starker bis sehr starker Fatigue. Starke Fatigue korreliert signifikant mit niedriger Lebensqualität (FERNANDES et al. 2006). 
Die Zahlen für tumorassozilerte Fatigue sind sehr hoch, lassen sich in anderen Studien aber durchaus bestätigen (ELSNER V. D. MALSBURG 2006; SMETS et al. 1993).

In Hypothese 1 wurde angenommen, dass der Anteil von Fatigue als Nebenwirkung von Tumorerkrankungen noch immer sehr hoch ist. Angesichts von fast $75 \%$ Fatigue unter den Tumorerkrankten und über 30\% mit starker Fatigue lässt sich Hypothese 1 als voll erfüllt ansehen. Fatigue stellt immer noch eine weit verbreitete Nebenwirkung von Tumorerkrankungen bzw. Tumortherapien dar.

\subsubsection{Diskussion der Ergebnisse des Dokumentationsbogens}

Die Altersstruktur der Gruppen war ähnlich. Die Trainingsgruppe war im Mittel drei Jahre jünger als die Kontrollgruppe. Daher wurden keine Effekte auf einen Altersunterschied zwischen den Gruppen zurückgeführt.

Als problematisch stellte sich die Geschlechterverteilung im Probandenkollektiv dar. Da der Anteil männlicher Probanden in beiden Gruppen nur bei ca. 10\% lag, war die Untergruppe „männliches Geschlecht“ stark unterrepräsentiert. Die Daten konnten daher nicht geschlechtsspezifisch ausgewertet werden. Da dies im Vorfeld nicht absehbar war, wurde die Studie auch nicht auf ein rein weibliches Probandenkollektiv beschränkt.

Die Ergebnisse der anthropometrischen Daten zeigen, dass ein hoher Anteil der Probanden übergewichtig war. 62,8\% der Probanden haben einen BMI $\geq 25$. Der BMI ist allerdings noch kein zuverlässiger Indikator für Übergewicht. In der heutigen Literatur werden zur Messung von Übergewicht eher Lean Body Mass oder Calipometrie als exaktes Instrument zur Messung von Übergewicht verwendet. Der Lean Body Mass gibt einen exakten Wert für die Masse an Fett und fettfreier Masse an. Leider war keine der genannten Messungen in der vorliegenden Untersuchung möglich.

Einige Patienten der Studie hatten einen derart erhöhten BMI, dass aller Wahrscheinlichkeit nach auch der Anteil an Körperfett deutlich erhöht war. 
Ein erhöhter Körperfettanteil zieht ein erhöhtes Krebsrisiko einiger Tumorerkrankungen nach sich (ROSENBERG et al. 2009; TEICHMANN 2002; ANDERSSON et al. 1997). Zudem gilt Übergewicht in Verbindung mit Bewegungsmangel als eine der Hauptursachen verschiedenster Zivilisationskrankheiten wie Diabetes mellitus oder Herz-Kreislaufkrankheiten (TEICHMANN, 2002).

Der Zeitraum der Diagnose war in der Kontrollgruppe im Mittel schon länger her als in der Trainingsgruppe. Der Anteil der Patienten, bei denen die Tumorerkrankung erst in den letzten 6 Monaten diagnostiziert wurde, war in der Trainingsgruppe signifikant höher als in der Kontrollgruppe. Demzufolge war auch der Anteil der Patienten höher, die zum aktuellen Zeitpunkt noch unter einer Behandlung standen. Es handelte sich hierbei in beiden Gruppen fast ausschließlich um Hormonbehandlungen. Werden die Ergebnisse der Fatigueerhebungsinstrumente genauer betrachtet, ist festzustellen, dass die Trainingsgruppe zu T1 in vielen Fällen höhere Werte zeigte als die Kontrollgruppe. Eine mögliche Erklärung dafür wurde in der durchschnittlichen größeren zeitlichen Nähe zum Erkrankungszeitpunkt vermutet.

Die sportlichen Vorerfahrungen der Probanden unterschieden sich zwischen den Gruppen nicht signifikant. In der Kontrollgruppe sank der Anteil der regelmäßig sportlich Aktiven vor der Rehabilitation stark ab. In der Trainingsgruppe konnte dieser Effekt nicht beobachtet werden. In den Sportkategorien unterschieden sich beide Gruppen nur marginal.

Die subjektive Selbsteinschätzung unterschied sich in beiden Gruppen während der drei Messzeitpunkte nur marginal. Es gab keine signifikanten Unterschiede zwischen den einzelnen Messterminen und Gruppen. Es konnte allerdings in beiden Gruppen während des Studienverlaufs eine Verschiebung der subjektiven Selbsteinschätzung in positive Richtung beobachtet werden. Zu den Messzeitpunkten T2 und T3 schätzten mehr Probanden ihre körperliche Leistungsfähigkeit als gut bzw. sehr gut ein. Hypothese 7 konnte aber nicht vollständig bestätigt werden, da die Entwicklung der subjektiven Selbsteinschätzung in beiden Gruppen fast identisch verlief. Eine Verbesserung der Selbsteinschätzung der Trainingsgruppe von T2 zu T3 konnte ebenso wenig wie in der Kontrollgruppe festgestellt werden. 
Unter Betrachtung aller Ergebnisse des Dokumentationsbogens bestätigte sich die Vergleichbarkeit beider Gruppen. Kontroll- und Trainingsgruppe unterschieden sich zwar geringfügig in einigen Bereichen der soziodemographischen und anthropometrischen Daten, insgesamt ähnelten sich die Ergebnisse allerdings so sehr, dass eine Vergleichbarkeit der Gruppen durchaus sinnvoll erschien.

\subsubsection{Diskussion von Angst und Depression (HADS)}

In einer Studie mit $\mathrm{n}=1792$ Probanden wurden Normwerte für Angst und Depression beim HADS in Abhängigkeit von Geschlecht und Alter aufgestellt. Dabei wurde für die Allgemeinbevölkerung in England ein Angstmittelwert von 6,14 und ein Depressionsmittelwert von 3,68 ermittelt (CRAWFORD et al. 2001). In Deutschland konnten Hinz et al. ähnliche Ergebnisse zeigen (HINZ et al. 2010).

In Kanada wurden Mittelwerte für Angst- und Depression bei $\mathrm{n}=3035$ Probanden kurz nach onkologischer Erkrankung ermittelt. Der Mittelwert für Angst lag bei 6,76 und der für Depression bei 4,30 (SELLICK \& EDWARDSON 2007).

Die Angstwerte dieser Studie lagen zum Messzeitpunkt T1 in der Kontrollgruppe mit 8,00 und in der Trainingsgruppe mit 8,47 deutlich höher als die oben genannten Werte. Bei den Depressionswerten zeigte sich ein ähnliches Bild. Die Mittelwerte für Depression lagen mit 6,20 in der Kontrollgruppe und 6,05 ebenfalls deutlich über den Werten von Sellick. In der Kontrollgruppe näherten sich die Angstwerte nach der Rehabilitation den Werten von Sellick an. In der Trainingsgruppe lagen sie sogar weit darunter. Die Angstwerte lagen in der Trainingsgruppe sogar unterhalb der von Crawford ermittelten Werte der Normalbevölkerung.

Ein ähnliches Ergebnis zeigte sich bei den Depressionswerten. Auch hier lagen die Werte der Kontrollgruppe nach der Rehabilitation in etwa auf dem Niveau von Sellick, während die Depressionswerte der Trainingsgruppe bis auf die Normwerte der Allgemeinbevölkerung nach Crawford absanken.

Dimeo et al. konnten zeigen, dass die Werte von Angst und Depression bei 32 Krebspatienten mit Fatigue im Verlauf eines dreiwöchigen Trainings der Ausdauer und ausgewählten Kräftigungs- und Koordinationsübungen sanken. Die Verbesserungen stellten sich allerdings nicht als signifikant heraus (DIMEO et al. 2008). 
In der hier vorliegenden Studie konnten in der Trainingsgruppe sowohl bei der Subskala Angst als auch bei der Subskala Depression signifikante Verbesserungen nachgewiesen werden, die auch während der nächsten drei Monate bei weiterführendem Krafttraining signifikant niedriger als die Ausgangswerte blieben.

Es wurde bereits nachgewiesen, dass sportliche Aktivität positive Wirkungen auf Depressionen hat. Dies wurde nicht nur bei Tumorpatienten nachgewiesen (DALEY 2008). Die Aussagen zu Angst und Depression der Hypothese 3 konnten durchaus bestätigt werden. Die Zeiteffekte sind in der Trainingsgruppe signifikant deutlicher als in der Kontrollgruppe. Angst und Depression sanken in der Trainingsgruppe während des Klinikaufenthalts ganz erheblich, während sich dieses Bild in der Kontrollgruppe nicht erkennen ließ.

\subsubsection{Diskussion der Fatigue (MFI, EORTC)}

Verschiedenste Studien belegen, dass körperliche Aktivität einen direkten Effekt auf Fatigue hat (COURNEYA 2010; MCAULEY et al. 2010; ALFANO et al. 2007, DIMEO 2001). Andere Studien in ähnlichem Kontext können herangezogen werden, um die Ergebnisse in einem übergreifenden Kontext darzustellen.

De Backer et al. zeigten in einer Studie, dass sich nach einem 18 Wochen andauernden hoch intensiven Krafttraining mit Ausnahme der Subskala Reduzierte Aktivität in allen Subskalen des MFI signifikante Veränderungen $(p<0,01)$ nachweisen ließen (DE BACKER et al. 2008).

Weitestgehend stimmen diese Ergebnisse mit denen der vorliegenden Studie überein. Allerdings war in einigen Subskalen der Trainingsgruppe die Signifikanz mit $p<0,0001$ noch wesentlich höher. Zudem ließen sich auch in der Subskala Reduzierte Aktivität signifikante Verbesserungen von T1 zu T3 nachweisen. Zum Teil ließen sich auch signifikante Veränderungen in der Kontrollgruppe nachweisen. Allerdings waren diese in keiner Subskala so deutlich wie in der Trainingsgruppe. Die Scores der einzelnen Subskalen der Trainingsgruppe waren zu T3 signifikant niedriger als in der Kontrollgruppe.

Ein fast identisches Resultat liefert die Subskala Fatigue des EORTC QLQ-C30. Auch hier konnten in der Trainingsgruppe hochsignifikante Unterschiede $(p<0,0001)$ zwischen T2 und T3 gegenüber T1 nachgewiesen werden. In der Kontrollgruppe 
konnten signifikante Unterschiede $(p \leq 0,05)$ zwischen T2 und T3 gegenüber T1 nachgewiesen werden.

Bereits drei Wochen nach Beginn der Studie zu T2 ließen sich in der Trainingsgruppe signifikante Unterschiede in allen Subskalen des MFI nachweisen.

Dies deckte sich mit den Ergebnissen einer Studie von Dimeo et al., der zumindest signifikante Unterschiede in den Subskalen Physische und Mentale Fatigue nachweisen konnte. (DIMEO et al. 2008).

Alle Subskalen ließen die Tendenz erkennen, dass die Trainingsgruppe zwischen T1 und T2 signifikant höhere Abnahmen der Scores aufwies als die Kontrollgruppe. In den weiteren drei Monaten zwischen T2 und T3 setzte sich dieser Trend fort.

Es kann angemerkt werden, dass die Ergebnisse der Studie bezüglich Fatigue eindeutig die Aussagen aus Hypothese 3 bestätigen. Der Verlauf der einzelnen Subskalen war allerdings in beiden Gruppen häufig ähnlich. Die Verbesserungen in der Trainingsgruppe waren jedoch durchweg höher.

\subsubsection{Diskussion der Lebensqualität (EORTC, FACIT)}

Die Lebensqualität wurde hauptsächlich mit dem EORTC QLQ-C30 und dem FACIT$\mathrm{F}$ gemessen.

Es muss erneut darauf aufmerksam gemacht werden, dass die Subskalen des EORTC in Funktionsskalen und Symptomskalen unterschieden werden.

Daher sollen diese Subskalen auch in der Diskussion differenziert betrachtet werden. Die Funktionsskalen des EORTC sind Global Health, Physical Functioning, Emotional Functioning, Social Functioning und Cognitive Functioning.

Alle Funktionsskalen zeigten innerhalb der Trainingsgruppe signifikante Unterschiede von T1 gegenüber T3. Eine Verbesserung der Lebensqualität durch körperliche Aktivität und insbesondere durch ein Krafttrainingsprogramm konnten auch andere Studien nachweisen (CRAMP et al. 2010; HAINES et al. 2010; VELTHUIS et al. 2010; DE BACKER et al. 2008; LIS et al. 2008).

Zenger et al. zeigten, dass Prostatakrebs eine starke psychische Belastung für die Patienten ist und somit einen erheblichen Einfluss auf die Lebensqualität hat (ZENGER et al. 2010). Emery et al. konnten zeigen, dass physische Gesundheit, Depressionssymptome und die emotionale Lebensqualität bei 
Brustkrebspatientinnen in den ersten 18 Monaten nach Behandlung von dem Maß der körperlicher Aktivität abhängen (EMERY et al. 2009). Segal et al. wiesen mit dem FACT-F nach, dass ein 24 Wochen andauerndes Krafttraining bei Prostatakrebspatienten positive Effekte auf Lebensqualität und Fatigue hatte (SEGAL et al. 2008).

Die Symptomskalen zeigten ein ebenso deutliches Ergebnis. Speziell in der Subskala Fatigue konnte in der Trainingsgruppe ein hochsignifikantes $(p<0,0001)$ Absinken des Scores vom Messzeitpunkt T1 zu T2 und T3 nachgewiesen werden. In der Kontrollgruppe konnte ebenfalls ein signifikantes $(p \leq 0,05)$ Absinken nachgewiesen werden, allerdings mit höherer Signifikanzgrenze.

Schlaflosigkeit nahm in der Trainingsgruppe von Messzeitpunkt T1 zu T3 signifikant ab. Es ist hinlänglich bekannt, dass die Schlafqualität ein wichtiger Parameter der Lebensqualität ist. Der Zusammenhang zwischen körperlicher Aktivität, Schlaflänge und Schlafqualität ist in der Literatur erläutert und in Studien belegt (REID et al. 2010; DE CASTRO TOLEDO GUIMARES et al. 2007).

Der Anstieg der Schlafstörungen während des gesamten Studienzeitraums in der Kontrollgruppe konnte nicht zulänglich erklärt werden. Daher konnte nur spekuliert werden. Ein möglicher Grund könnte die ungewohnte Umgebung in der Klinik gewesen sein. Allerdings waren die Voraussetzungen der Trainingsgruppe identisch. Nur wenige Erkenntnisse ließen sich aus der Subskala Schmerz ziehen. Es konnten in keiner der beiden Gruppen signifikante Veränderungen zwischen den Messzeitpunkten nachgewiesen werden.

Kopp et al. konnten nachweisen, dass die Ergebnisse des FACIT-F zum Teil mit denen des EORTC QLQ-C30 korrelieren. Speziell in Bereichen der körperlichen Funktionsfähigkeit liegen hohe Korrelationskoeffizienten vor. Im Bereich der emotionalen und sozialen Funktion sind die Korrelationskoeffizienten eher gering und weniger aussagekräftig (KOPP et al. 2000).

Der FACIT-F zeigte in beiden Gruppen hochsignifikante $(p<0,0001)$ Unterschiede in der Subskala Körperliches Wohlbefinden. Bereits vom Messzeitpunkt T1 zu T2 stiegen die Scores hochsignifikant an und blieben bis T3 auf diesem Niveau nahezu konstant. Die Subskalen Funktionsfähigkeit und Seelisches Wohlbefinden zeigten nur in der Trainingsgruppe signifikante Effekte. In der Kontrollgruppe waren die Scores beider Subskalen nahezu konstant. 
Bei der Subskala Verhältnis zu Freunden und Familie ließen sich in beiden Gruppen keine Effekte nachweisen.

Sowohl in der Kontroll- als auch in der Trainingsgruppe konnten im Verlauf der stationären Rehabilitation positive Effekte auf verschiedene Lebensqualitätsparameter nachgewiesen werden. Die Ausprägung dieser Effekte sowie deren Nachhaltigkeit nach dem stationären Aufenthalt waren in der Trainingsgruppe deutlicher als in der Kontrollgruppe.

Mit der Verbesserung der Lebensqualität konnten einige Aussagen von Hypothese 3 bestätigt werden. Der Verlauf der einzelnen Subskalen war zum Teil in beiden Gruppen ähnlich. Die positiven Veränderungen der einzelnen Subskalen waren jedoch in der Trainingsgruppe durchweg höher.

Hypothese 3 konnte hinsichtlich der Aussagen über die unterschiedlichen Verbesserungen in den einzelnen Bereichen von Angst, Depression, Fatigue und Lebensqualität voll bestätigt werden. Hinsichtlich der unterschiedlichen Entwicklung der Scores konnte die Hypothese nicht vollständig bestätigt werden.

\subsubsection{Diskussion der Fatiguerhythmik}

Die Fatiguerhythmik wurde in beiden Gruppen mit dem konzipierten mehrstufigen LASA-Fragebogen getestet, wobei täglich zu fünf Messzeitpunkten ein Fatiguewert abgefragt wurde. Eine Unterscheidung in „Lärchen“ und „Eulen“, wie in der Literatur zum Teil vorgenommen wurde während der Untersuchung nicht durchgeführt. Hierfür würde die Gruppengröße der notwendigerweise zu bildenden Untergruppen nicht ausreichen. Zudem konnten nur einige wenige Datensätze ermittelt werden, die in den Morgenstunden eine höherer Fatigueschwere als am Abend aufwiesen.

Beide Gruppen zeigten zum Messzeitpunkt T1 ein ähnliches Bild des Tagesfatigueverlaufs. Die Fatiguewerte stiegen im Tagesverlauf kontinuierlich an und erreichten um 21 Uhr ihre Höchstwerte. Der Tagesverlauf zeigte somit eindeutig, dass die Patienten in den Abendstunden erschöpfter waren als am Tagesanfang. Hypothese 4 ließ sich somit bestätigen. Mit später werdender Tageszeit steigen die Fatiguewerte, wie in Hypothese 4 angenommen, kontinuierlich an. 
Curran et al. zeigten in einer Studie mittels Ecological Momentary Assessment ähnliche Ergebnisse bei Frauen mit Brustkrebs. Die Ergebnisse zeigen einen ähnlichen Anstieg der Fatigue im Tagesverlauf und einen Höchstwert zu der spätesten Messzeit von 21 Uhr (CURRAN et al. 2004).

Während die Ausgangswerte des Fatigueverlaufs beider Gruppen zu T1 noch sehr ähnlich waren, differierten diese zu T2 und T3 zunehmend.

In der Kontrollgruppe ließen sich zwischen den Messzeitpunkten T1, T2 und T3 nur an einer Stelle signifikante Veränderungen nachweisen. Tendenziell sank der Fatiguewert zu den morgendlichen Messzeiten 8 Uhr und 11 Uhr stärker als zu den späteren. Die Messungen zu den Zeiten $14 \mathrm{Uhr}, 17 \mathrm{Uhr}$ und $21 \mathrm{Uhr}$ zeigten nahezu keine Veränderung der Fatiguewerte in der Kontrollgruppe. Die ursprüngliche Situation steigender Fatiguewerte im Tagesverlauf blieb auch zu T2 und T3 bestehen.

In der Trainingsgruppe hingegen konnten zu nahezu allen täglichen Messzeiten signifikante Veränderungen zwischen $\mathrm{T} 1, \mathrm{~T} 2$ und $\mathrm{T} 3$ nachgewiesen werden.

Die Fatiguewerte sanken signifikant von T1 zu T2. 8 Uhr und 14 Uhr konnten sogar signifikante Senkungen der Fatiguewerte von T2 zu T3 nachgewiesen werden.

Trotzdem blieb das ursprüngliche Bild steigender Fatiguewerte im Tagesverlauf auch zu T2 und T3 bestehen. Allerdings lagen die Fatiguewerte zu T2 und T3 auf einem niedrigeren Niveau als zu T1. Hypothese 5 konnte nur zum Teil bestätigt werden. Die Fatiguewerte der Trainingsgruppe sanken zu allen Tageszeiten signifikant von T1 zu T2. Auch zwischen T2 und T3 konnten noch deutliche, zum Teil sogar signifikante Senkungen der Fatiguescore zu verschiedenen Tageszeiten beobachtet werden. In der Kontrollgruppe konnte zwar ein Abfall der Fatiguewerte zwischen T1 und T2 beobachtet werden, allerdings war dieser nur an einer Stelle signifikant. Zu den späteren Tageszeiten $14 \mathrm{Uhr}, 17 \mathrm{Uhr}$ und $21 \mathrm{Uhr}$ konnten nur sehr geringe, keinesfalls signifikante, Veränderungen zwischen den Messzeitpunkten nachgewiesen werden. Zwischen T2 und T3 konnten nur noch minimale Veränderungen nachgewiesen werden.

Vergleichsstudien zur Fatigueverlaufsdiagnostik im Zusammenhang mit sportlicher Aktivität konnten nicht ermittelt werden. 


\subsection{Diskussion der Leistungsdiagnostik}

\subsubsection{Diskussion der Ausdauertestergebnisse}

\section{6-Minuten Gehtest}

Der 6-Minuten Gehtest wird in der Literatur als einfache Messmethode beschrieben, um die aerobe Ausdauer zu messen (YUEN \& SWORD 2007). Der Test kann bei verschiedensten Diagnosen zur Messung der aeroben Ausdauer angewendet werden und zeigt eine hohe Test-Retest Reliabilität (RIES et al. 2009; VIS et al. 2009 ).

Unter Beachtung einiger Testparameter korreliert der 6-Minuten Gehtest wie in Abschnitt 5.5.2.2 beschrieben mit dem VO2max Test (OPASICH et al. 2001; ZUGCK et al. 2000; LUCAS et al. 1999; FAGGIANO et al. 1997; CAHALIN et al. 1996; RILEY et al. 1992; LIPKIN et al. 1986).

Es konnten in beiden Gruppen signifikante Unterschiede zwischen T1 und T2 nachgewiesen werden. Die Leistungssteigerung zwischen T1 und T2 war allerdings in der Trainingsgruppe deutlich höher als in der Kontrollgruppe. Die Signifikanzwerte bestätigten in der Trainingsgruppe ein hochsignifikantes $(p<0,0001)$ Ergebnis. In der Kontrollgruppe ließ sich im Mittel ein Leistungszuwachs von 31 Metern nachweisen. In der Trainingsgruppe war es mit 64 Metern eine mehr als doppelt so lange Strecke. $\mathrm{Ob}$ in dieser kurzen Zeit zwischen T1 und T2 eine tatsächliche Steigerung der aeroben Ausdauer vorliegen kann, muss angezweifelt werden. Als Erklärungsansatz für kurzzeitige Veränderungen dienen eher neuronale Veränderungen. Die Zusammenarbeit zwischen zentralem Nervensystem und Muskulatur verbessert sich, was sich in einer Mehrrekrutierung von Muskelfasern äußert. Es kommt zu einer Verbesserung des Zusammenspiels im Muskel (intramuskulär) und zwischen mehreren Muskeln (intermuskulär). Die Trainingswissenschaft spricht von intra- und intermuskulärer Koordination. Diese kann sich schon nach kurzer Zeit positiv auf die Leistungsfähigkeit auswirken. Bewegungsabläufe werden ökonomischer. Die Folge ist eine Verringerung der Herzarbeit bei gegebener physikalischen Leistung der Skelettmuskulatur, bzw. eine Steigerung der physikalischen Leistung bei gegebener Herzarbeit. Es kann davon ausgegangen werden, dass erste physiologische 
Anpassungen periphere Systeme betreffen und erst später das kardiopulmonale System angepasst wird (HOLLMANN \& HETTINGER, 2000). Grosser et al. unterscheiden vier Anpassungsstufen (Anlage 13).

Trotzdem ist die größere Leistungssteigerung der Trainingsgruppe durchaus mit dem erhöhten Trainingsumfang durch das zusätzliche Übungsprogramm erklärbar. Sowohl physiologische als auch neuronale trainingsbedingte Anpassungsvorgänge werden durch erhöhten Trainingsumfang bei bestehender Intensität beschleunigt. Hypothese 2 kann insofern als erwiesen betrachtet werden, als das sich beim 6Minuten Gehtest in beiden Gruppen signifikante Verbesserungen der Ausdauer nachweisen ließen. Aufgrund der Aussagen der Anpassung nach Grosser et al. kann weiterhin davon ausgegangen werden, dass die Anpassungserscheinungen, die zu der Verbesserung geführt haben, lediglich neuronalen Ursprungs sind.

\section{PWC-130 Fahrradergometertest}

Der PWC Fahrradergometertest lieferte kaum verwertbare Ergebnisse. Für die Zielgruppe des Tests wurde der PWC130 ausgewählt, da PWC 150 oder PWC 170 für die Patientenzielgruppe aufgrund der hohen zu erreichenden Pulswerte ungeeignet schienen (STEMPER, 1988).

Dennoch erreichten nur etwa 60\% der Patienten den Zielpuls von 130 Schlägen pro Minute. Viele Patienten waren aufgrund ihres Alters, mangelnder physischer Leistungsfähigkeit oder Medikamenteneinnahme nicht in der Lage, sich bis zum Zielpuls zu belasten. Eine Berechnung der relativen Leistung,

wie sie Stemper vorgibt, war dadurch nicht möglich.

Daher wurde der PWC Test zum Einen nach gefahrener Zeit und zum Anderen nach der erreichten Wattstufe ausgewertet. In den Ergebnissen beider Auswertungsansätze konnten in keiner Gruppe signifikante Effekte nachgewiesen werden. Die Verbesserungen der Trainingsgruppe in der Zeitanalyse waren geringfügig höher als die der Kontrollgruppe.

Auch bei diesem Testverfahren muss darauf hingewiesen werden, dass aufgrund der Kürze der Trainingsphase mit physiologischen Veränderungen kaum zu rechnen war. Die Verbesserungen müssen vorwiegend aus einer Ökonomisierung der muskulären Funktion resultieren. 
Ein weiteres Problem folgte aus der fehlenden Berechnung der relativen Leistung und den intuitiv gewählten Auswertungsvarianten. Durch diese war die Vergleichbarkeit zu anderen Studien nicht mehr ausreichend gewährleistet.

Somit scheinen die PWC Ergebnisse nur eine sinnvolle Ergänzung zu den Ergebnissen des 6-Minuten Gehtests zu sein. Die eigene Aussagekraft wird jedoch als gering eingeschätzt. Insofern spielten die Ergebnisse des PWC 130 zur Bestätigung von Hypothese 2 nur eine Nebenrolle.

\subsubsection{Diskussion der Krafttestergebnisse}

Die kraftdiagnostischen Untersuchungen zeigten einen Zeiteffekt von T1 zu T2 vor allem in der Trainingsgruppe. Das Bewegungs- und Sportprogramm der Klinik in Verbindung mit dem Trainingsprogramm der Studie wirkte sich in vielen Fällen positiv auf die isometrische Maximalkraft der Arme und Beine aus. Es konnten zwar in beiden Gruppen keine signifikanten Effekte nachgewiesen werden, trotzdem war eine Steigerung der Maximalkraft $\mathrm{zu}$ beobachten. Die Verbesserungen der Trainingsgruppe waren dabei deutlich höher als die der Kontrollgruppe. Die isometrische Maximalkraft der Arme verbesserte sich in der Trainingsgruppe durchschnittlich um 15,7 N. Bei den Beinen waren es im Durchschnitt 46,0 N. In der Kontrollgruppe waren die Verbesserungen mit durchschnittlich 5,1 $\mathrm{N}$ bei den Armen und 31,9 N bei den Beinen nicht so deutlich. Zusätzlich konnte beobachtet werden, dass in der Kontrollgruppe im Mittel 59,8\% der Probanden ihre isometrische Maximalkraft steigerten. In der Trainingsgruppe war der Anteil mit 80,2\% deutlich höher.

Verschiedene Autoren konnten in Studien belegen, dass Krafttraining während onkologischer Erkrankungen die Kraftfähigkeit steigert und darüber hinaus zahlreiche weitere Parameter wie Lebensqualität und Fatigue positiv beeinflusst (CRAMP et al. 2010). Courneya et al. konnten zeigen, dass durch Krafttraining sogar während der Chemotherapie positive Effekte auf Kraftfähigkeit und andere Parameter zu erzielen sind (COURNEYA et al. 2007).

Zusätzlich wurde festgestellt, dass der Anteil der Patienten, die während der Rehabilitation inre isometrische Maximalkraft steigerten in der Trainingsgruppe 
ebenfalls höher war als in der Kontrollgruppe. Dies könnte aus dem zusätzlichen Krafttraining der Trainingsgruppe resultieren.

Zusammenfassend mussten die leistungsdiagnostischen Daten differenziert betrachtet werden. Zum Einen konnten in allen leistungsdiagnostischen Testverfahren Leistungssteigerungen beobachtet werden. In der Trainingsgruppe waren diese stets deutlicher als in der Kontrollgruppe, was auf den erhöhten Trainingsumfang der Trainingsgruppe zurückzuführen war. Trotzdem ist die Zeitspanne zwischen den Messterminen mit drei Wochen zu knapp gewesen, um leistungssteigernde physiologische Veränderungen hervorzurufen. Die gestiegenen Leistungen in den leistungsdiagnostischen Testverfahren wurden daher eher auf neuronale Effekte zurückgeführt. Diese waren weit weniger deutlich als bei der Messung der Ausdauer. Der Grund hierfür lag in den isolierten Testbedingungen in denen einzelne Muskelgruppen getestet wurden. Eine positive neuronale Wirkung im Sinne einer Verbesserung der intermuskulären Koordination bzw. der neuronalen Ansteuerung durch das Zentralnervensystem kam dabei nicht in so großem Maße zum Tragen wie bei der Ausdauer.

Ein positiver Effekt der Leistungsdiagnostik war allerdings die Förderung der Motivation. Zum Messtermin T2 haben sich viele Probanden deutlich in ihrer Leistung gegenüber T1 verbessert und bekamen so eine Bestätigung für die sportlichen Leistungen, die sie während ihres Rehabilitationsaufenthaltes erbracht haben. Daraus resultiert eine gesteigerte Motivation für weiterführende sportliche Aktivität. Aufgrund der vorhandenen Zeiteffekte konnte Hypothese 2, auch was die Kraftentwicklung betraf, als erfült betrachtet werden. Die positiven Effekte waren eindeutig auf eine Veränderung der Koordination zurückzuführen. 


\subsection{Diskussion der Auswirkungen des tatsächlichen Trainingsumfangs auf die verschiedenen Untersuchungsparameter}

Im Fatigueverlauf zeigten sich keine signifikanten Unterschiede zwischen den gebildeten Subgruppen. Es wurde allerdings deutlich, dass die Subgruppe Überdurchschnitt die geringste Ausprägung von Fatigue zu jeder Tageszeit hatte. Allerdings müssen auch die Ausgangswerte aller Subgruppen zu T1 beachtet werden. Aus Abbildung 70 wird ersichtlich, dass die Ausgangswerte zu T1 in der Subgruppe Überdurchschnitt niedriger waren als in der Subgruppe Unterdurchschnitt. Bei der Schlafqualität zeigten sich zu T3 keine signifikanten Unterschiede zwischen den Subgruppen. Im Verlauf der Untersuchung konnten nur in der Subgruppe Unterdurchschnitt signifikante Veränderungen in der Schlafqualität von T1 zu T3 nachgewiesen werden. In den anderen beiden Subgruppen war dies nicht der Fall. Zudem war der Ausgangswert in der Subgruppe Unterdurchschnitt niedriger als in den anderen beiden Subgruppen.

In der Subskala Aktivitätslevel konnten signifikante Unterschiede zwischen der Subgruppe Unterdurchschnitt und der Subgruppe Überdurchschnitt nachgewiesen werden. Bei relativ ähnlichen Ausgangswerten zu T1 verbesserte sich nur die Subgruppe Überdurchschnitt signifikant.

In nahezu allen untersuchten Bereichen der Lebensqualität, Fatigue, Angst und Depression zeigte sich ein deutliches Schema. Die höchste Ausprägung eines Symptoms zum Messzeitpunkt T3 fand sich in fast allen Fällen in der Subgruppe Unterdurchschnitt, während sich die niedrigste Ausprägung in der Subgruppe Überdurchschnitt fand. Die Subgruppe Durchschnitt fand sich in vielen Fällen zwischen den anderen beiden. Zum Teil sind die Unterschiede zwischen den Subgruppen Unterdurchschnitt und Überdurchschnitt signifikant. Die Ausgangswerte der einzelnen Subskalen zu T1 waren in vielen Fällen in der Subgruppe Unterdurchschnitt am höchsten. Signifikante Unterschiede ließen sich vermehrt in der Subgruppe Überdurchschnitt finden. Dieses Bild zeigte sich sowohl in den Angst- und Depressionsskalen des HADS als auch in den Fatiguesubskalen des MFI. Beim EORTC zeigte sich ein identisches Bild in den Symptomskalen Fatigue und Schmerz. In den Funktionsskalen des EORTC und des FACIT-F war es genau umgekehrt. Hier zeigten die Ergebnisse meist den niedrigsten Score in der Subgruppe Unterdurchschnitt und den höchsten in der Subgruppe Überdurchschnitt. Die 
Ausgangswerte zu T1 waren in der Subgruppe Unterdurchschnitt häufig niedriger als in den anderen beiden Subgruppen.

Die Einteilung der Subgruppen nach tatsächlichem Trainingsumfang war intuitiv, brachte jedoch das Ergebnis, dass die Patienten mit hohem Trainingsumfang deutlich, zum Teil sogar signifikant bessere Ergebnisse in nahezu allen untersuchten Parametern der Lebensqualität und Fatigue aufwiesen. Allerdings waren auch die Ausgangswerte dieser Patienten in den meisten Fällen schon besser als bei denen der anderen beiden Subgruppen.

Trotzdem konnte angenommen werden, dass ein hoher Trainingsumfang positive Auswirkungen auf Parameter der Fatigue, Lebensqualität, Angst und Depression hatte. 


\section{Zusammenfassung}

Nach wie vor zählt tumorbedingte Fatigue zu den häufigsten Beschwerden onkologischer Patienten, da durch diese Nebenwirkung die Lebensqualität stark eingeschränkt wird.

Mit der durchgeführten Interventionsstudie wurden verschiedene Zielstellungen erfüllt.

Zum Ersten wurde eine Vollerhebung der Fatigue über den gesamten Zeitraum der Datenerhebung durchgeführt. Hierbei wurde deutlich, dass tumorbedingte Fatigue bei einem sehr hohen Anteil onkologischer Patienten festzustellen war. Die Relevanz zur Entwicklung und Durchführung therapeutischer Interventionen zu tumorassoziierter Fatigue erscheint daher noch immer sehr hoch.

Zum Zweiten sollte die Wirksamkeit eines rehabilitativen Krafttrainings gegenüber tumorassoziierter Fatigue und Lebensqualität überprüft werden. Diese konnte sowohl während der stationären Rehabilitation ( $\mathrm{T} 1$ - $\mathrm{T} 2$ ) als auch in der dreimonatigen postrehabilitativen Heimtrainingsphase (T2 - T3) nachgewiesen werden. Die zu allen drei Messzeitpunkten ausgefüllten Fragebögen zu Fatigue und Lebensqualität (MFI, EORTC QLQ-C30, FACIT-F), sowie Angst und Depression (HADS) ergaben fast durchweg positiv signifikante $(p \leq 0,05)$ Zeiteffekte in der Trainingsgruppe. In einigen Bereichen waren die Effekte sogar hochsignifikant $(p<0,0001)$. In der Kontrollgruppe konnten nur vereinzelt positiv signifikante Effekte nachgewiesen werden.

Die leistungsdiagnostischen Daten wurden mit verschiedenen Ausdauer- bzw. Krafttests erhoben. Zur Erhebung der aeroben Ausdauerleistungsfähigkeit wurden der 6-Minuten Gehtest und der PWC 130 verwendet. Die isometrischen Maximalkraftmessungen wurden durch ein semi-objektives Messverfahren mittels einer Kraftmessdose durchgeführt. Die Änderungen der leistungsdiagnostisch erhobenen Daten sind in erster Linie auf inter- und intramuskuläre Koordinationseffekte zurückzuführen, da in einer Zeitspanne von ca. 3-4 Wochen leistungssteigernde physiologische Veränderungen nur von geringer Relevanz sind. Ein weiteres Ziel der Arbeit bestand darin, Erkenntnisse über den Tagesverlauf der Fatigue zu gewinnen und nachzuvollziehen, welchen Einfluss körperliche Aktivität in Form eines rehabilitativen Krafttrainings auf diesen hat. Es war durchaus erkennbar, dass es in beiden Gruppen einen ähnlichen Fatigueverlauf über den Tag gab. In beiden Gruppen stieg die Fatigue ausgehend von einem niedrigen Ausgangswert am 
Morgen über den Tag kontinuierlich an, bis am Abend ein Maximum erreicht wurde. Somit konnte für tumorassoziierte Fatigue ein zirkadianrhythmischer Verlauf nachgewiesen werden. In Verbindung mit dem rehabilitativen Krafttraining konnte gezeigt werden, dass die Kurve der Fatigue in der Trainingsgruppe schon während der stationären Rehabilitation, aber auch danach, signifikant abflachte. In der Kontrollgruppe konnte dieser Effekt nicht nachgewiesen werden. Eine zirkadianrhythmische Platzierung der Übungseinheit im Tagesablauf konnte in der Studie aufgrund der Organisationsstruktur der stationären Rehabilitation nicht gewährleistet werden. Hier könnte ein weiteres Forschungsinteresse anknüpfen.

Erkenntnisse vieler anderer Studien konnten in dieser Arbeit bestätigt werden. Funktionelles Krafttraining wirkt sich während und nach der stationären onkologischen Rehabilitation in vielen Bereichen positiv auf Leistungsfähigkeit, Lebensqualität und Fatigueparameter aus.

Zusätzlich konnte die Studie in einigen Bereichen innovative Anregungen über zukünftiges Forschungspotential geben. Der nachgewiesene zirkadianrhythmische Tagesverlauf der Fatigue verspricht innovatives Forschungspotential, um Rehabilitation und Genesungsprozess weiter zu optimieren und bestmögliche Therapieergebnisse zu erreichen. 


\section{Ausblick}

Die Wirkungen von körperlicher Aktivität auf tumorassoziierte Fatigue sind in zahlreichen Studien belegt. Übungsprogramme sind eine sinnvolle Intervention für onkologische Patienten, um diesen eine Möglichkeit zu einem selbstständigen Training zu geben. Für diese Programme erscheinen funktionelle Kräftigungsübungen als optimale Ergänzung zu einem gemäßigten aeroben Ausdauertraining. Es sollte jedoch im Vorfeld einen gewissen Zeitraum unter Aufsicht trainiert werden. Dadurch kann Intensität und Umfang der Belastung optimal und individuell auf den Patienten eingestellt werden. Eine weitere Betreuung der Patienten in regelmäßigen Abständen erscheint durchaus sinnvoll.

In einem nächsten Schritt könnten die Erkenntnisse des zirkadianrhythmischen Fatigueverlaufs in die Trainingsplanung einbezogen werden. Nach zirkadianrhythmischen Aspekten erschien ein Training gegen $18 \mathrm{Uhr}$ in vielerlei Hinsicht optimal. Zu dieser Zeit erreichen alle zirkadian ablaufenden physiologischen Prozesse einen Bereich, in dem die körperliche Leistungsfähigkeit und somit auch die Trainingseffektivität optimal ist. Ob dies auch für onkologische Patienten mit tumorassoziierter Fatigue gilt, wäre eine Interessante Fragestellung für eine Folgestudie. Es gilt zu untersuchen, zu welchen Fatiguewerten ein Training maximale Effekte erzielen kann. Macht Training bei starker Fatiguebelastung Sinn? Welche Belastungsintensität und -umfänge erzielen größtmöglichen Erfolg bei geringen Nebenwirkungen? Studien zu diesen Fragestellungen wurden noch nicht durchgeführt. Erkenntnisse dieser Art könnten eine Optimierung der stationären Rehabilitation onkologischer Patienten nach trainingswissenschaftlichen Aspekten ermöglichen. Zur Organisation des Tagesablaufs der Patienten könnten trainingswissenschaftliche Erkenntnisse über die zeitliche Platzierung sportlicher Inhalte herangezogen werden, um die Adaptionseffekte für die Patienten zu maximieren.

Die Effekte für die Patienten könnten eine schnellere Genesung und Wiedereingliederung in das soziale Leben und den Arbeitsprozess bedeuten. Diese birgt eine schnelle Zurückerlangung eines Höchstmaßes an Lebensqualität und bedeutet nicht zuletzt eine Verkürzung des Genesungsprozesses. 


\section{Literaturverzeichnis}

Aaronson, N., Ahmedzal, S., Berman, B., Bullinger, M., Cull, A., DUEZ, N. (03/1993). The European Organisation for Reaserch and Treatment of Cancer QLQ-C30: A quality of life instrument for use in international clinical trials in oncology. Journal of the National Cancer Institute (85) , S. 365-376.

AHLBerg, K., Ekman, T., GAStON-JOHANNSON, F., \& MOCK, V. (2003). Assessment and management of cancer-related fatigue in adults. The Lancet (362), S. $640-650$.

Alfano, C., Wilder SMITH, A., IRWIN, M., BOWEN, D., SOREnSEN, B., REEVE, B. (2007). Physical Activity, long-term symptoms, and physical healthrelated quality of life among breast cancer survivors: A prospective analysis. Journal of Cancer Survivors , S. 116-128.

ANCOLI-ISRAel, S., LIU, L., MARLER, M., PARKeR, B., JoNES, V., SADLER, G. (03/2006). Fatigue, sleep and circadian rhythms prior to chemotherapy for breast cancer. Supportive care in cancer (14), S. 201-209.

Andersson, S., WolK, A., Bergström, R., AdAMI, H., ENGHOLM, G., ENGLUND, A. (1997). Body size and prostate cancer: a 20-year follow-up study among 135006 construction workers. Journal of the National Cancer Institute (03) , S. 385-389.

ARENDT, J. (1993). Some Effects of Light and Melatonin on Human Rhythms. Light and biological Rhythms in man (WETTERBERG, L.) , S. 203-235.

ASTRAND, P., RODAHL, K., DAHL, H., \& STROMmE, S. (2003). Textbook of Work Physiology - Physiological Bases of Exercises. Leeds: Human Kinetics Europe.

BARDWELL, W., \& ANCOLI-ISRAEL, S. (2008). Breast Cancer and Fatigue. Sleep Medicine Clinics (03) , S. 61-71.

BAUMANN, F., KRAUT, L., SCHÜLE, K., BLOCH, W., \& FAUSER, A. (02/2010). A controlled randomized study examining the effects of exercise therapy on patients undergoing haematopoietic cell transplantation. Bone Marrow Transplant , S. 355362.

BaumanN, F., Leskaroski, A., MÜLleR, S., WAJdA, S., KRAKOWSKIRoOSEN, H., BLOCH, W. (2010). Effekte eines dreimonatigen Krafttrainingsprogramms auf die Fatigue-Symptomatik bei Mammakarzinompatientinnen während der zytostatischen Therapie. Onkologie (33) , S. 199. 
BJelland, I., DAHL, A., HAUG, T., \& NECKELmANN, D. (2002). The validity of the hospital anxiety and depression scale: an updated literature review. Journal of psychosomatic research (02) , S. 69-77.

CELLA, D. (1997). The functional Assessment of Cancer Therapy-Anemia (FACTAn) scale: A new Tool for Assessment of Outcomes in Cancer Anemia Fatigue. Seminars in Hematology (34), S. 13-19

CourneyA, K., SEgAL, R., Gelmon, K., ReID, R., MACKeY, J., FRIEDENREICH, C. (2007). Six-month follow-up patient-rated outcomes in a randomized controlled trial of exercise training during breast cancer chomotherapy. Alberta: University of Alberta.

CourneyA, K., Segal, R., MACKey, J., Gelmon, K., ReID, R., FRIEDENREICH, C. (2007). Effects of aerobic and resistance exercise in breast cancer patients receiving adjuvant chemotherapy: a multicenter randomized controlled trial. Journal of Clinical Oncology (25) , S. 4396-4404.

CRAMP, F., \& DANIEL, J. (2008). Exercise improves cancer-related fatigue. Cochrane Database of Systematic Review.

CRAMP, F., JAMES, A., \& LAMBERT, J. (2010). The effects of resistance training on quality of life in cancer: a systematic literature review and meta-analysis. New York: Springer-Verlag.

CraWford, J., HenRY, J., CROMBIE, C., \& TAYLOR, E. (2001). Normative data for the HADS from large non-clinical sample. British Journal of Clinical Psychology (09) , S. 429-434.

CURRAN, S., BEACHAM, A., ANDRYKOWSKI, M. (10/2004). Ecological Momentary Assessment of Fatigue following Breast Cancer Treatment. Journal of Behavioral Medicine (27) , S. 425-444.

DALEY, A. (2008). Exercise and depression: a review of reviews. Journal of Clinical Psych. Medicine (06) , S. 140-147.

De BaCker, I., VReugdenhil, G., NiJziel, M., Kester, A., VAN BRedA, E., \& SCHEP, G. (2008). Long-term follow-up after cancer rehabilitation using highintensity resistance training: persistant improvement of physical performance and quality of life. British Journal of Cancer (99) , S. 30-36.

De Castro Toledo Guimares, L., De Carvalho, L., Yanaguibashi, G., \& FERnANDES Do PRADO, G. (2007). Physically active elderly woman sleep more and better than sedentary woman. Sao Paulo: UNIFESP.

DE MARÉES, H. (1979). Sportphysiologie. Köln: Troponwerke. 
DEMITRI, G., KRIS, M., WASDE, J., DEGOS, L., \& CELLA, D. (1998). Quality of life benefit in chemotherapy patients treated with epoetin alfa is indipendent of desease response or tumor type: results from a prospective community oncology study. Clinical Oncology (16) , S. 3412-3425.

DeNK, H., PACHE, D., \& SChAlleR, H. (2003). Handbuch Alterssport. Schorndorf: Hofmann Verlag.

DEUTSCHE KREBSgeSELLSCHAFT (2003). Fatigue, wenn Müdigkeit quälend wird. Frankfurt/Main: (42) Eigendruck.

DICKHUTH, H. (2000). Einführung in die Sport- und Leistungsmedizin. Schorndorf: Hofmann Verlag.

DIMEO, F. (2001). Effects of Exercise on Cancer-Related Fatigue. Cancer (92) , S. 1689-1693.

Dimeo, F., SchWARTZ, S., Wesel, N., VoIGT, A., \& THIEL, E. (2008). Effects of an endurance and resistance exercise program on persistent cancer-related fatigue after treatment. Berlin: Department of Hematology, Oncology and Transfusion Medicine, Charité Berlin.

DRESCHER, H., \& SIMON, G. (1989). Maximale Leistung und PWC 170. In D. BÖNINNG, K. BRAUMANN, M. BUSSE, N. MAASEN, \& W. SCHMIDT, Sport, Rettung oder Risiko für die Gesundheit? (S. 404-408). Köln: Deutscher Ärzte Verlag.

ELSNER V. D. MALSBURG, M. (2006). Untersuchung der Einsetzbarkeit und Effektivität eines strukturierten Übungsprogramms in der Rehabilitation bei Brustkrebspatientinnen mit Fatigue. Göttingen: Cuvillier Verlag.

Emery, C., HAe-ChUng, Y., Frierson, G., Peterson, L., \& SOOYeON, S. (2009). Determinants of physical activity among woman treated for breast cancer in a 5-year longitudinal follow-up investigation. Psychooncology (04) , S. 377-386.

Fernandes, R., StOne, P., ANDReWS, P., MORGAN, R., \& ShaRma, S. (09/2006). Comparison between fatigue, sleepdisturbance and circadian rhythm in cancer inpatients and healthy volunteers: evaluation of diagnostic criteria for cancerrelated fatigue. Journal of Pain and Symptom Management (32) , S. 245-254.

FleChtNeR, H.\& BOtOOMLEY, A. (2003). Fatigue and Quality of Life: Lessons from the Real World. The Oncologist (8) , S. 5-9

FRoBÖSE, I., NEllessen-MARTENS, G., \& WILKE, C. (2010). Training in der Therapie. München: Elsevier $\mathrm{GmbH}$.

GABRILOVE, J., CLEELAND, C., \& LIVINGSTON, R. (2001). Clinical evaluation of once-weekly dosing of epoetin alfa in chemotherapy patients: Improvements in hemoglobin and quality of life are similar to three times weekly dosing. Clinical Oncology (19) , S. 2875-2882. 
GROSS, G. (1983). Circadian Rhythms and Circadian System. In J. Mendlewicz, \& H. VAN PRAAG, Biological Rhythms and Behaviour (S. 1-9). Basel: Karger Verlag.

GRosSER, M., STARISCHKA, S., \& ZIMMERMANN, E. (2008). Das neue Konditionstraining. München: BLV Buchverlag GmbH \& Co. KG.

haines, T., Sinnamon, P., Wetzig, N., lehman, M., Walpole, E., Pratt, T. (2010). Multimodal exercise improves quality of life of woman being treated for breast cancer, but at what cost? Randomized trial with economic evaluation. Chicago.

HAMILTON, D., \& HAENNEL, R. (03/2000). Validity and reliability of the 6-minute walk test in a cardiac rehabilitation population. Journal of Cardiopulm. Rehabilitation (20) , S. 156-164.

HERMANN, C. (1997). International experiences with Hospital anxiety and depression scale - a review of validation data and clinical results. Journal of psychosomatic research (42), S. 17-41.

HERMANN, C., BUSS, U., \& SNAITH, R. (1995). HADS-D - Hospital Anxiety and Depression Scale - Deutsche Version: Ein Fragebogen zur Erfassung von Angst und Depressivität in der somatischen Medizin. Bern: Huber Verlag.

HERMANN, C., SCHOLZ, K., \& KREUZER, H. (1991). Psychologisches Screening von Patienten einer kardiologischen Akutklinik mit einer deutschen Fassung der "Hospital anxiety and depression" (HAD) Skala. Psychotherapie, Psychosomatik, medizinische Psychologie (41), S. 83-92.

Hinz, A., KRAuss, O., Hauss, J., HÖCKEl, M., KORTMANN, R., STOLZENBURG, J. (2010). Anxiety and depression in cancer patients compared with general population. European Journal of Cancer (19) , S. 522-529.

HOHMANN, A.; LAMES, M.; LETZELTER, M. (2002). Einführung in die Trainingswissenschaft. Wiebelsheim: Limpert Verlag.

HOllmANN, W., \& HETTINGER, T. (2000). Sportmedizin. Stuttgart: Schattauer Verlag.

HOLLMANN, W., \& STRÜDER, H. (2009). Sportmedizin. Stuttgart : Schattauer Verlag.

JACobsen, P., Donovan, K., SMAll, B., JiM, H., MUNSTeR, P., \& ANDRYKOWSKY, M. (2007). Fatigue after Treatment for early stage breast cancer. Cancer (10) , 1851-1859. 
JeAN PierRe, P., FiguerA-Moseley, C., KOHLI, S., Fiscella, K., PALESH, O., \& MORROW, G. (2007). Assessment of Cancer-Related Fatigue: Implications for Clinical Diagnosis and Treatment. The Oncologist , S. 11-21.

KLINIKPROSPEKT, WICKER GRUPPE (2004). Sonnenbergklinik. Kassel: Druck \& Verlag Baden.

KOPP, M., SCHWEIGKOFLER, H., HOLZNER, B., NACHBAUR, D., NIEDERWIESER, D., FLEISCHHACKER, W. (2000). EORTC QLQ-C30 and FACTBMT for the measurement of quality of life in bone marrow transplant recipients: a comparison. European Journal of Haematology (08) , S. 97-103.

KURZROCK, R. (2001). The role of cytokines in cancer-related fatigue. Cancer (94), S. $1684-1688$.

LIPKIN, D., SCRIVEN, A., CRAKE, T., \& POOLE-WILSON, P. (1986). Six-minute walk test for assessing exercise capacity in chronic heart failure. British Medicine Journal (03) , 653-655.

LIS, A., QUIST, M., ANDERSEN, C., Moller, T., HeRRStedT, J., KRONBORG, D. (2008). Effect of a multimodal high intensity exercise intervention in cancer patients undergoing chemotherapy: randomised controlled trial. Research BMJ: Institute of Plublic Health, University of Copenhagen.

LOCKE, D., DeCKer, P., SloAN, J., BROWN, P., MAleC, J., ClARK, M. (2007). Validation of single-item linear analog scale assessment of quality of life in neuro oncology patients. Journal of pain and symptom management (12) , S. 628638.

MARKWORTH, P. (2006). Sportmedizin: Physiologische Grundlagen. Hamburg: Rowohlt Verlag.

MCAUley, E., White, S., RogerS, L., MOTL, R., \& COURNeYA, K. (2010). Physical activity and fatigue in breast cancer and multiple sclerosis: physical mechanisms. Psychosomatic Medicine (01) , S. 88-96.

MinTON, O., \& STONE, P. (2009). A systematic review of the scales used for the measurement of cancer-related fatigue. Annals of Oncology (01) , 17-25.

MOORE-EdE, C., SULZMAN, F., \& FULLER, C. (1982). The Clock that time us Physiology of the Circadian Timing System. Harvard: The President and Fellows of Harvard College.

OSCHÜTZ, H. (1991). Chronobiologie im Sport - Zur Entwicklung der sportlichen Leistungsfähigkeit unter Berücksichtigung der zirkadianen Rhythmik. Leistungssport (21) , S. 12-15. 
OSCHÜTZ, H. (1998). Zirkadianrhythmische Aspekte muskulärer Beanspruchung und wiederherstellungsfördernder Maßnahmen. In L. ZICHNER, M. ENGELHARDT, \& J. FREIWALD, Die Muskulatur: Sensibles, integratives und meßbares Organ (S. 173-180). Nürnberg: Novatis Pharma Verlag.

PfeifeR, K., VOGT, L., \& BANZER, W. (2003). Messpositionen und deren Reproduzierbarkeit zur Erfassung der isometrischen Maximalkraft verschiedener Muskelgruppen . Gesundheitssport und Sporttherapie (19) , S. 5-8.

PHILLIPS, M. L. (2009). Circadian Rhythms: Of owls, larks and alarm clocks. Nature (03), 142-144

PORTER, M., VANDERVOORT, A., \& LEXELL, J. (1995). Aging of human muscle: structure, function and adabtability. Scand. Journal of Medicine and Science in Sports (06) , S. 129-142.

PORTER, M., VANDERVOORT, A., \& LEXELL, J. (1995). Aging of Human Muscle: Structure, Function and Adaptability. Sandinavian Journal of Medicine and Science in Sports (5) , S. 129.

PREUSS, P., \& PREUSS, M. (2008). Silver Generation-Krafttraining für Senioren. Köln: Heel Verlag.

RADLINGER, L. (1998). Rehabilitative Trainingslehre. Stuttgart, New York: Thieme Verlag.

RASCHKA, C., \& VOGEL, M. (2010). Herzsport. Wiebelsheim: Limpert Verlag.

RAY, M., ROGERS, L., TRAMMELL, R., \& TOTH, L. (2008). Fatigue and Sleep during Cancerand Chemotherapy: Translational Rodent Models . Comparative Medicine (58) , 234-245.

REFINETTI, R. (2000). Circadian Physiologie. Boca Raton, Florida: CRC Press LLC.

ReID, K., BARON, K., LU, B., NAYlOR, E., WolfE, L., \& ZEE, P. (2010). Aerobic exercise improves self-reported sleep and quality of life in older adults with insomnia. Sleep Medicine , S. 934-940.

REMME, W., \& SWEDBERG, K. (2001). Task Force for the Diagnosis and Treatment of Chronic Heart Failure, European Society of Cardiology: Guidelines for the diagnosis and treatment of chronic heart failure. European Heart Journal (22) , S. 1527-1560.

RIES, J., ECHTERNACH, J., NOF, L., \& GAGNON BLODGETT, M. (2009). Testretest reliability and minimaldetectable change scores for the timed up \& go test, the six-minute walk test, and gait speed in people with Alzheimer disease. Physical Therapy (06) , S. 569-579. 
RILeY, M., MCPARLAND, J., StANFORD, C., \& NiCHOLLS, D. (06/1992). Oxygen consumption during corridor walk testing in chronic cardiac failure. European Heart Journal (13) , S. 789-793.

RosenbeRG, L., CZENE, K., \& HALL, P. (2009). Obesity and poor breast cancer prognosis: an illusion because of hormone replacement therapy? British Journal of Cancer (05) , S. 1486-1491.

RÜHL, J., \& SCHUBERT, V. (2003). Funktionelles Fitnesskrafttraining. Aachen: Meyer\&Meyer Verlag.

SCHMIDT, R., \& THEWS, G. (2000). Physiologie des Menschen. Berlin: Springer Verlag.

SCHNABEL, G., HARRE, H., \& KRUG, J. (2008). Trainingslehre Trainingswissenschaft: Leistung - Training - Wettkampf. Aachen: Meyer \& Meyer Verlag.

SCHÜLE, K. (2000). Grundlagen der Sporttherapie: Prävention, ambulante und stationäre Rehabilitation. München: Urban und Fischer Verlag.

SChWABerger, G., Pessenhofer, H., SAUSeng, N., \& SCHMid, P. (1983). Die Beziehung der PWC 170 zum aerob-anaeroben Übergang (Laufbandergometrie Untersuchung). In H. MELLEROWITZ, \& I. FRANZ. Erlangen: Perimed Verlag.

SCHWARZ, R., KRAUSS, O., \& HINZ, A. (2003). Fatigue in general population. Onkologie (04), S. 140-144.

Segal, R., Reid, R., Courneya, K., Sigal, R., KenNy, G., Prud Homme, D. (2008). Randomized Controlled Trial of Resistance or Aerobic Exercise in Man receiving Radiation Therapy for Prostate Cancer. Journal of Clinical Oncology (27) , S. 344-351.

SELLICK, S. M., \& EDWARDSON, A. (2007). Screening new cancer patients for psychological distress using the hospital anxiety and depression scale. PsychoOncology (16) , S. 534-542.

Servaes, P., Van Der Werf, S., Prins, J., Verhagen, S., \& BLEIJENBERG, G. (01/2001). Fatigue in desease-free cancer patients compared with fatigue in patients with chronic fatigue syndrom. Supportive care in cancer (9) , S. 11-17.

Servaes, P., Verhagen, C., \& BleiJenBeRG, G. (2002). Fatigue after breast cancer and in chronic fatigue syndrom. Similarities and differences. Journal of Psychosomatic Research (52) , S. 27-43.

Singer, S., Kuhnt, S., Götze, h., hauss, A., LiebmanN, A., KRAUSS, O. (2009). Hospital anxiety and depression scale cutoff scores for cancer patients in 
acute care. London: Department of Epidemiology and Population Health, University of London.

SINZ, R. (1980). Chronopsychophysiologie - Chronobiologie und Chronomedizin. Berlin: Akademie Verlag.

SMETS, E., GARSSEN, B., BONKE, B., \& DE HAES, J. (1995). The Multidimensional Fatigue Inventory - Psychometric Qualities of an Instrument to assess Fatigue. Journal of Psychometric Research (39) , 315-325.

SmetS, E., GARSSEN, B., Schuster-UitTerhoeVE, A., \& De HAES, J. (08/1993). Fatigue in Cancer Patients. British Journal of Cancer , S. 220-224.

SPENCE, R., HEESCH, K., \& BROWN, W. (2009). Exercise and cancer rehabilitation: A systematic review. Cancer Treatment Reviews (36) , 185-194.

STEMPER, T. (1988). Gesundheit-Fitness-Freizeitsport. Köln: Bund-Verlag.

StOne, P., RichaRdSON, A., ReAM, E., SMith, A., KERR, D., \& KEARNEY, N. (2000). Cancer-related fatigue: inevitable, unimportant and untreatable? Results of a multi-centre patient survey. Cancer Fatigue Forum. Annals of Oncology (08) , 971975.

TEICHMANN, J. (2002). Onkologische Rehabilitation: Evaluation der Effektivität stationärer onkologischer Rehabilitationsmaßnahmen. Rehabilitation (41) , S. 53-63.

TITTEL, K. (1994). Beschreibende und funktionelle Anatomie des Menschen. Jena: Fischer Verlag.

TRINTSCHER, K. (1967). Biologie und Information - Eine Diskussion über Probleme der biologischen Thermodynamik. Leipzig: B.G. Teubner Verlagsgesellschaft.

Velthuis, M., MAY, A., KOPPEJAN-RensenbRINK, R., GIJSEN, B., BREDA, E., DE WIT, G. (2010). Physical activity during cancer treatment (PACT) Study: design of a randomized clinical trial. BioMed Central.

VIS, J., ThOONSEN, H., DUfFElS, M., DE BRUIN-BON, R., HUISMAN, S., VAN DIJK, A. (2009). Six-minute walk test in patients with down syndrome: validity and reproduceibility. Archives of Physical Medicine and Rehabilitation (08) , 1423-1427.

WAGNER, L., \& CELLA, D. (2004). Fatigue and Cancer: causes, prevalence and treatment approaches. British Journal of Cancer (91) , 822-828.

Webster, K., CEllA, D., \& YOST, K. (2003). The Functional Assessment of Chronic IIIness Therapy (FACIT). Measurement System: properties, applications and interpretation. Center on outcomes, Research and Education (CORE). Evanston: Northwestern University.

WEINECK, J. (2010). Optimales Training. Balingen: Spitta Verlag GmbH. 
WEIS, J., BARTSCH, H., \& WOLTEMATE, I. (2002). Fatigue und ihre Bedeutung für die Rehabilitation von Tumorpatienten: Rehabilitationsbedarf, Interventionen und Forschungsaufgaben. In J. WEIS, \& H. BARTSCH, Fatigue bei Tumorpatienten. Eine neue Herausforderung für Therapie und Rehabilitation. (S. 108-120). Basel, Freiburg: Karger Verlag.

WURSTLER, K., \& KELLER, E. (1985). Endokrine Regulation und Frauenhochleistungssport. Berlin : Springer-Verlag.

YUEN, H., \& SWORD, D. (2007). Home based exercise to allivate fatigue and improve functional capacity among breast cancer survivors. Journal of Allied Health (36) , S. 257-275.

Zenger, M., LehmanN-laUe, A., Ju, S., Schwalenberg, T., Ried, A., \& HINZ, A. (2010). The relationship of quality of life and distress in prostate cancer patients compared to the general population. GMS Psycho-Social-Medicine (7) .

ZIMMERMANN, K. (2000). Gesundheitsorientiertes Krafttraining: Theorie - Empirie - Praxisorientierung. Schorndorf: Hofmann Verlag. 


\section{Anhang}

\section{Anlage 1: Einwilligungserklärung zur Studie:}

Prof. Dr. M.E. Heim, Sonnenberg-Klinik, Hardtstr. 13, 3337242 Bad Sooden-Allendorf

\section{„Rehabilitatives Krafttraining zur Behandlung tumorbedingter Fatigue unter Einbeziehung des Energieverlaufs der Patienten.“}

Name des Patienten Zimmernr.

Ich wurde über Wesen, Bedeutung und Tragweite des oben genannten Forschungsprojektes aufgeklärt. Ich habe die Patienteninformation gelesen und verstanden. Ich hatte die Möglichkeit, Fragen zu stellen, habe die Antworten verstanden und akzeptiere sie. Ich wurde über den möglichen Nutzen der Studie informiert.

Ich hatte ausreichend Zeit, mich zur Teilnahme an dieser Untersuchung zu entscheiden und weiß, dass die Teilnahme an dieser Untersuchung freiwillig ist. Ich weiß, dass ich jederzeit und ohne Angabe von Gründen diese Zustimmung widerrufen kann, ohne dass sich dieser Entschluss auf die spätere Behandlung durch meinen Arzt auswirken wird.

Insbesondere bin ich damit einverstanden, dass Klinikmitarbeiter Diagnosen, Medikation und Behandlungsergebnisse aus der Krankenblattakte entnehmen und unter einer Forschungsnummer an das wissenschaftliche Forschungsinstitut übermitteln. Ich entbinde die Klinikmitarbeiter insoweit von ihrer Schweigepflicht.

Mir wurde versichert, dass keine personenbezogenen Angaben (Name, Geburtsdatum, Adresse oder sonstige Angaben, die Rückschlüsse auf meine Person zulassen) an Dritte weitergegeben werden und dass im Zusammenhang mit dieser Untersuchung erhobene Daten gelöscht werden, sobald sie für die weitere wissenschaftliche Auswertungen nicht mehr erforderlich sind.

Hiermit erkläre ich mich bereit, an der geplanten Untersuchung unter den im Aufklärungsschreiben genannten Voraussetzungen teilzunehmen. 


\section{Anlage 2: Informationsschreiben für die Teilnehmer zur Studie}

\section{„Rehabilitatives Krafttraining zur Behandlung tumorbedingter Fatigue unter Einbeziehung des Energieverlaufs der Patienten.“}

\section{Wer führt die Studie durch?}

Matthias Heim ist Doktorand der Sportwissenschaften an der Georg-August Universität Göttingen. Die Untersuchung der Beeinflussung tumorbedingter Fatigue (Erschöpfung) unter Einbeziehung einer Energieverlaufsstudie durch Rehabilitatives Krafttraining ist Thema seiner Doktorarbeit.

Die Sonnenberg-Klinik Bad-Sooden Allendorf unter Leitung von Prof. M. E. Heim tritt als Partner der Universität auf und unterstützt in diesem Sinne das Forschungsvorhaben.

\section{Warum wird die Studie durchgeführt?}

In einer Studie der Deutschen Krebshilfe konnte gezeigt werden, dass über $50 \%$ aller Krebspatienten tumorbedingte Fatigue (Erschöpfung) als die einschränkendste Nebenwirkung der Krankheit empfinden. Da bisher über die Ursachenstruktur und Therapierung nur unzureichende Kenntnisse vorhanden sind, besteht in diesem Bereich ein erhöhtes Forschungsinteresse. Diese Studie soll dazu beitragen die Therapierung der Krankheit weiterzuentwickeln.

\section{Wie wird gemessen?}

Als Messverfahren werden ausschließlich leistungsdiagnostische Tests und Fragebögen verwendet. Es werden keine invasiven Untersuchungen durchgeführt. Das bedeutet für Sie, dass keine Blut- oder Gewebeentnahmen während der Studie erforderlich werden. Sie werden während der Rehabilitation lediglich Kraft- und Ausdauertestverfahren unterzogen und durchlaufen im Zuge Ihrer Rehabilitation ein speziell abgestimmtes Trainingsprogramm. Außerdem werden einige empirische Daten mit Hilfe von wissenschaftlichen Fragebögen erfasst. 


\section{Dauer der Studie?}

Die Studie umfasst für Sie den Zeitraum Ihres Rehabilitationsaufenthalts in der Sonnenberg-Klinik Bad-Sooden Allendorf und eine dreimonatige Nachlaufzeit. Währen dieser drei Monate bekommen Sie ein spezielles Übungsmanual mit Hilfe dessen Sie auch weiterhin gezielte Übungen durchführen und Ihre Ergebnisse dokumentieren sollen. Nach drei Monaten findet abschließend eine postalische Befragung statt.

Die während der Studie erhobenen Daten werden anonymisiert und ausschließlich zu Studienzwecken genutzt. Sie unterliegen streng dem Datenschutz. Eine Weitervergabe an Dritte ist ausgeschlossen.

\section{Interesse der Doktorarbeit:}

Mit der Studie soll die Wirkung eines Rehabilitativen Krafttrainings auf Ihre körperliche Leistungsfähigkeit untersucht werden. Außerdem sollen Trainingsreize unter bestimmten Aspekten des Energieverlaufs untersucht werden. Ziel der Studie ist letztlich ein für die Rehabilitation und für Sie zu Hause praktikables hochwirksames Training zu entwickeln, dass Ihnen im Anschluss an Ihre Krankheit eine schnellstmögliche und effektive Möglichkeit bietet, Ihre Leistungsfähigkeit und Ihr Wohlbefinden zu steigern.

Kontakt:

Matthias Heim

Annastraße 16

37075 Göttingen

Tel.: 0176/62886040

mailto: matze_heim82@web.de 
Anlage 3: Fatigue-LASA

Name des Patienten:

Zimmernummer:

\section{Wie erschöpft fühlen Sie sich?}

Bitte machen Sie ein Kreuz auf der Stelle der Skala, die nach Ihrer eigenen Einschätzung Ihren aktuellen Erschöpfungszustand beschreibt!

keine Erschöpfung

starke Erschöpfung

(;)

(2)

\begin{tabular}{ccccccccccc}
\hline I & I & I & I & I & I & I & I & I & I & I \\
0 & 1 & 2 & 3 & 4 & 5 & 6 & 7 & 8 & 9 & 10
\end{tabular}

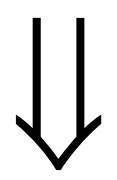

Bitte geben Sie den oberen Teil des Zettels in jedem Fall im Stationszimmer ab.

Falls das von Ihnen gemachte Kreuz auf der Skala im Bereich von 4 - 10 liegt, möchten wir Sie gerne am Mittwoch nach Ihrer Anreise um 16.00 Uhr zu einer Informationsveranstaltung in den Gruppenraum 3 (1.

Etage gegenüber der Klinikleitung) einladen. 
Anlage 4: Fragebogen zur Tagesrhythmik Fatigue

Name des Patienten:

Zimmernummer:

Wie erschöpft fühlen Sie sich zu bestimmten Tageszeiten?

Bitte machen Sie bei jeder Uhrzeit ein Kreuz an der Stelle, die Ihren aktuellen Erschöpfungszustand zu dieser Uhrzeit beschreibt.

Hierbei beschreibt 0 den Zustand von keiner Erschöpfung und 10 den stärksten Grad der Erschöpfung.

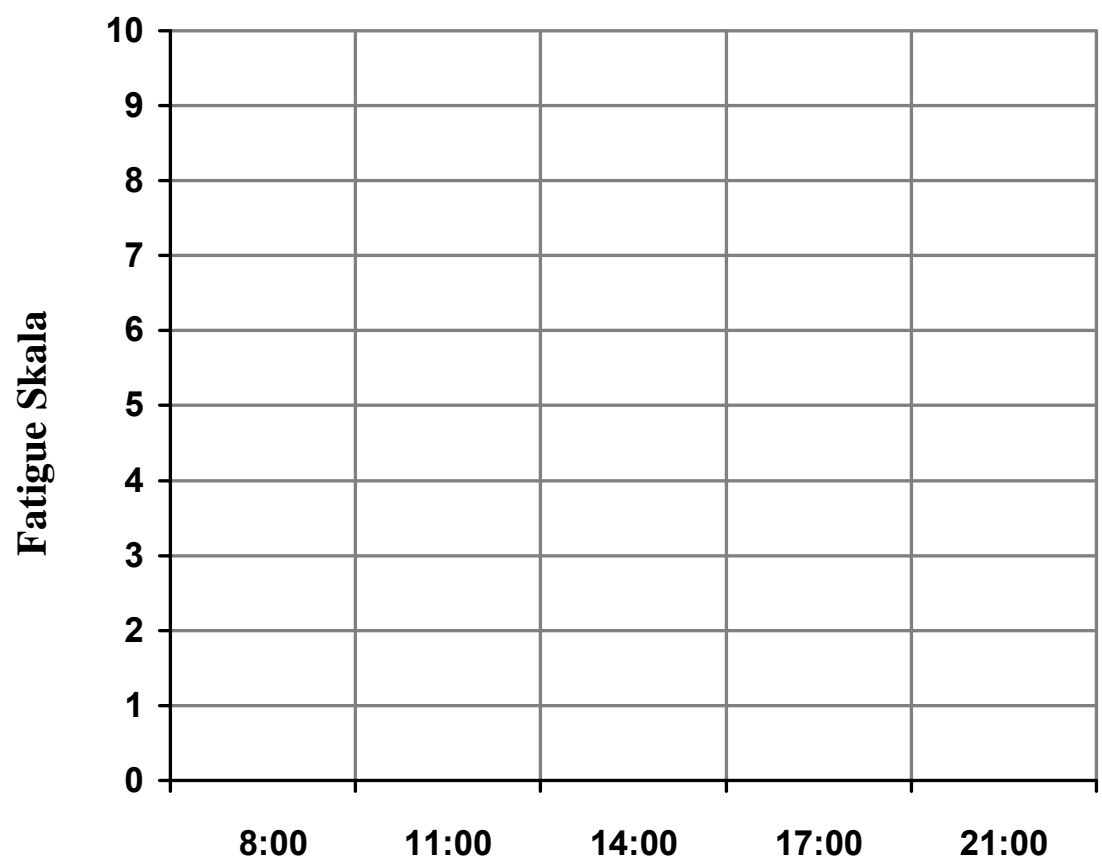

Uhrzeit

Schlafqualität (Bitte ankreuzen):

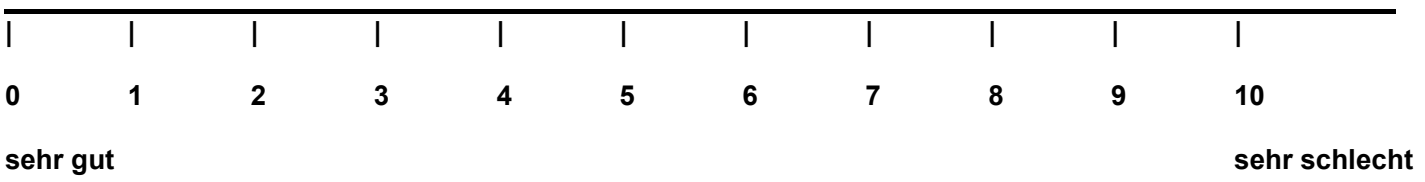

Aktivitätslevel (Bitte ankreuzen):

\begin{tabular}{lllllllllll}
\hline I & I & I & I & I & I & I & I & I & I & I \\
0 & 1 & 2 & 3 & 4 & 5 & 6 & 7 & 8 & 9 & 10 \\
sehr gut & & & & & & & & & & sehr schlecht
\end{tabular}

\section{Vielen Dank!}




\section{Anlage 5: Dokumentationsbogen vor der Rehabilitation (T1)}

Feststellung der Krankheitsbilder, durchlaufenen Therapien und sportlichen Vorerfahrungen.

Prof. Dr. med. M. Heim

Chefarzt der Sonnenbergklinik Bad Sooden-Allendorf

Erhebungszeitpunkt: Beginn der stationären Rehabilitation (T1)

Name:

Teilnehmer-Nr.:

Dieser Fragebogen wurde an folgendem Datum ausgefüllt:

$\overline{\text { Tag }} \quad \overline{\text { Monat }} \overline{\text { Jahr }}$ 
Soziale Anamnese und allgemeine Fragen zur Person:

Alter:

Aktuelle berufliche Situation:

o Hausfrau/ Hausmann

- Ganztagsbeschäftigung

o Teilzeitbeschäftigung

o Selbstständig

o Rentner/in, Ruhestand

Sonstiges: (bitte angeben)

Geschlecht

Größe:

Gewicht:

$\begin{array}{ll}\text { Raucher } \mathrm{O} & \text { Nichtraucher } \mathrm{O}\end{array}$

Wenn Raucher: Seit wie viel Jahren?___ Seit welchem Lebensjahr?

Wie viele Zigaretten pro Tag?

\section{Fragen zur Erkrankung und Behandlung}

Diagnose:

Datum der Erkrankung (Diagnose):

Monat:

Jahr:

Datum der Erstbehandlung: Monat:

Jahr:

Welche Art der Erstbehandlung wurde durchgeführt? (bitte ankreuzen)

Operation

$\mathrm{O}$

Chemotherapie

O

Hormontherapie

O

Strahlentherapie

O

Sonstige Therapieformen? 
Laufen zur Zeit noch spezifische Behandlungen? Ja $\mathrm{O}$ Nein $\mathrm{O}$ Wenn ja, welche?

$\begin{array}{ll}\text { Chemotherapie } & \text { O } \\ \text { Hormontherapie } & \text { O } \\ \text { Schmerztherapie } & \text { O } \\ \text { Antikörpertherapie } & \text { O }\end{array}$

Andere

Wann wurde die letzte Behandlung (Chemotherapie, Strahlentherapie, Hormontherapie)abgeschlossen?

Nehmen Sie zur Zeit Schmerzmittel ein?
$\mathrm{Ja} \quad \mathrm{O}$
Nein $\mathrm{O}$

Wenn ja, welche?

Leiden Sie zur Zeit an Schlafstörungen?
$\mathrm{Ja}$
$\mathrm{O}$
Nein $\mathrm{O}$

Wenn ja, handelt es sich eher um:

Einschlafstörungen

Durchschlafstörungen

$\mathrm{O}$

Nehmen Sie zur Zeit Schlafmittel ein?
$\mathrm{Ja}$
$\mathrm{O}$
Nein $\mathrm{O}$

Wenn ja, welche?

Nehmen Sie zur Zeit Nahrungsergänzungsmittel ein?
Ja
$\mathrm{O}$
Nein $\mathrm{O}$

Wenn ja, welche? 
Stationäre Rehabilitation aufgrund der Behandlung bereits zu einem früheren Zeitpunkt?
Ja $\mathrm{O}$
Nein $\mathrm{O}$

Wenn ja, wann?

\section{Fragen zur sportlichen Aktivität}

Betrieben Sie vor Ihrer Erkrankung Sport?

Nein, gar nicht

Unregelmäßig

O

Ja, regelmäßig

$\mathrm{O}$

$\mathrm{O}$

Wenn ja: Welche Sportarten?

Aus welcher Motivation treiben Sie Sport?

Gesundheit

Spaß

0

Ausgleich

$\mathrm{O}$

Geselligkeit

$\mathrm{O}$

$\mathrm{O}$

Andere

Haben Sie vor Ihrem Rehabilitationsaufenthalt Sport betrieben?

Nein, gar nicht

0

Unregelmäßig

O

Ja, regelmäßig

O 
Wenn ja: Welche Sportarten?

Wie würden Sie Ihre aktuelle körperliche Leistungsfähigkeit einschätzen?

Sehr gut $O$

Gut

$\mathrm{O} \quad$ Weniger gut $\mathrm{O}$

Schlecht

$\mathrm{O}$ seit wann?

Std. pro Woche

seit wann?
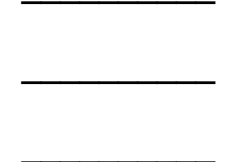
Anlage 6: Dokumentationsbogen nach der Rehabilitation (T2)

Fragen zur subjektiven Empfindung nach der Rehabilitationsmaßnahme.

Prof. Dr. med. M. Heim

Chefarzt der Sonnenbergklinik Bad Sooden-Allendorf

Erhebungszeitpunkt: Ende der stationären Rehabilitation (T2)

Name:

Teilnehmer-Nr.:

Dieser Fragebogen wurde an folgendem Datum ausgefüllt:

$\overline{\text { Tag }} \quad \overline{\text { Monat }} \quad \overline{\text { Jahr }}$ 


\section{Fragen zum Befinden nach der Rehabilitation:}

Gewicht:

Haben Sie das Gefühl, dass sich Ihre körperliche Leistungsfähigkeit während der Rehabilitation gesteigert hat?

Ja $\mathrm{O} \quad$ Nein $\mathrm{O}$

Wie würden Sie Ihre aktuelle körperliche Leistungsfähigkeit einschätzen?

Sehrgut $O$ Gut $O$ Weniger gut $O$ Schlecht $O$

In wie weit hat sich Ihr Wohlbefinden während der Rehabilitation geändert?

viel schlechter $\quad \mathrm{O}$ etwas schlechter $\mathrm{O}$ gar nicht $\mathrm{O}$

etwas besser $\quad 0 \quad$ viel besser $\quad 0$

Denken Sie, dass die Änderung auch auf die sportliche Aktivität zurückzuführen ist?

Ja $\quad \mathrm{N} \quad$ Nein $\mathrm{O}$

Empfinden Sie die sportlichen Aktivitäten im Rahmen Ihres Aufenthaltes in der Sonnenberg-Klinik als angenehm und nützlich?

Ja $\quad \mathrm{N} \quad$ Nein $\mathrm{O}$

Hatten Sie nach dem Krafttraining Schmerzen am Muskelapparat (Beine, Rumpf, Rücken, Arme)?

Ja $\quad \mathrm{N}$ Nein $\mathrm{O}$

Hatten Sie nach dem Training auf dem Fahrradergometer Schmerzen am Muskelapparat (Beine, Gesäß, Rücken)?

Ja $\quad \mathrm{N}$ Nein $\mathrm{O}$

Wenn ja, wo? 
Aus welcher Motivation treiben Sie Sport?

Gesundheit

Spaß

$\mathrm{O}$

Ausgleich

$\mathrm{O}$

Geselligkeit

$\mathrm{O}$

$\mathrm{O}$

Andere

\section{Fragen zur Erkrankung und Behandlung}

Nehmen Sie zur Zeit Schmerzmittel ein?

$$
\text { Ja } \mathrm{O} \text { Nein } \mathrm{O}
$$

Wenn ja, welche?

Leiden Sie zur Zeit an Schlafstörungen?

Ja $\mathrm{O}$ Nein $\mathrm{O}$

Wenn ja, handelt es sich eher um: Einschlafstörungen

$\mathrm{O}$

Durchschlafstörungen

$\mathrm{O}$

Nehmen Sie zur Zeit Schlafmittel ein?
Ja $\mathrm{O}$
Nein $\mathrm{O}$

Wenn ja, welche?

\section{Vielen Dank!}


Anlage 7: Dokumentationsbogen drei Monate nach der Rehabilitation (T3)

Fragen zur subjektiven Empfindung drei Monate nach der Rehabilitationsmaßnahme

Prof. Dr. med. M. Heim

Chefarzt der Sonnenbergklinik Bad Sooden-Allendorf

Erhebungszeitpunkt: Drei Monate nach Rehabilitation (T3)

Teilnehmer-Nr.:

Dieser Fragebogen wurde an folgendem Datum ausgefüllt:

$\overline{\text { Tag }} \quad \overline{\text { Monat }} \quad \overline{\text { Jahr }}$ 


\section{Soziale Anamnese und spezielle Fragen zur Person}

Gewicht:

$\begin{array}{lll}\text { Raucher } \mathrm{O} & \text { Nichtraucher } \mathrm{O}\end{array}$

Wenn Raucher: Seit wie viel Jahren?__ Seit welchem Lebensjahr?

Wie viele Zigaretten pro Tag?

\section{Fragen zur Erkrankung und Behandlung}

Laufen zur Zeit noch spezifische Behandlungen? Ja $\mathrm{O} \quad$ Nein $\mathrm{O}$

Wenn ja, welche?

Chemotherapie $\quad 0$

Hormontherapie $\quad \mathrm{O}$

Schmerztherapie $\quad 0$

Antikörpertherapie

O

Andere

Wann wurde die letzte Behandlung (Chemotherapie, Strahlentherapie, Hormontherapie)abgeschlossen?

Nehmen Sie zur Zeit Schmerzmittel ein?
$\mathrm{Ja} \quad \mathrm{O}$
Nein $\mathrm{O}$

Wenn ja, welche?

Leiden Sie zur Zeit an Schlafstörungen?
Ja $\mathrm{O}$
Nein $\mathrm{O}$

Wenn ja, handelt es sich eher um: Einschlafstörungen

$\mathrm{O}$ 
Nehmen Sie zur Zeit Schlafmittel ein?

$$
\text { Ja } \mathrm{O} \text { Nein } \mathrm{O}
$$

Wenn ja, welche?

Nehmen Sie zur Zeit Nahrungsergänzungsmittel ein?
Ja
O
Nein $\mathrm{O}$

Wenn ja, welche?

\section{Fragen zur sportlichen Aktivität}

Aus welcher Motivation treiben Sie Sport?

Gesundheit

$\mathrm{O}$

Spaß

Ausgleich

$\mathrm{O}$

Geselligkeit

$\mathrm{O}$

$\mathrm{O}$

Andere

Haben Sie nach Ihrem Rehabilitationsaufenthalt Sport betrieben?

Nein, gar nicht

0

Unregelmäßig

$\mathrm{O}$

Ja, regelmäßig

$\mathrm{O}$

Wenn ja: Welche Sportarten?

Std. pro Woche

seit wann?

Haben Sie das Gefühl, dass sich Ihre körperliche Leistungsfähigkeit nach der Rehabilitation gesteigert hat?

Ja $\quad \mathrm{N}$ Nein $\mathrm{O}$ 
Denken Sie, dass die Änderung auch auf die sportliche Aktivität zurückzuführen ist?

Ja $\mathrm{O}$ Nein $\mathrm{O}$

Hatten Sie nach dem Krafttraining Schmerzen am Muskelapparat (Beine, Rumpf, Rücken, Arme)?

Ja $\quad \mathrm{N}$ Nein $\mathrm{O}$

Wie würden Sie Ihre aktuelle körperliche Leistungsfähigkeit einschätzen?

$\begin{array}{llllll}\text { Sehrgut } O & \text { Gut } & O & \text { Wenigergut } & \end{array}$

In wie weit hat sich Ihr Wohlbefinden nach der Rehabilitation geändert?

viel schlechter $\quad \mathrm{O}$ etwas schlechter $\mathrm{O}$ gar nicht $\mathrm{O}$

etwas besser $\quad 0 \quad$ viel besser $\quad 0$ 
Anlage 8: Testprotokoll 6-Minuten Gehtest

\section{Testprotokoll 6-Minuten Gehtest}

\begin{tabular}{|l|l|l|l|}
\hline \multicolumn{2}{|l|}{ Name: } & Testdatum: & Abreise: \\
\hline Geburtsdatum: & Größe: & Gewicht: \\
\hline Zeit: & & Gehstrecke: & Puls: \\
\hline Ausgang: & & & \\
\hline 2 Min. & & & \\
\hline 4 Min. & & & \\
\hline 6 Min. & & & \\
\hline Nach 2 Min. & & & \\
\hline
\end{tabular}


Anlage 9: Testprotokoll PWC 130

\section{Testprotokoll PWC 130}

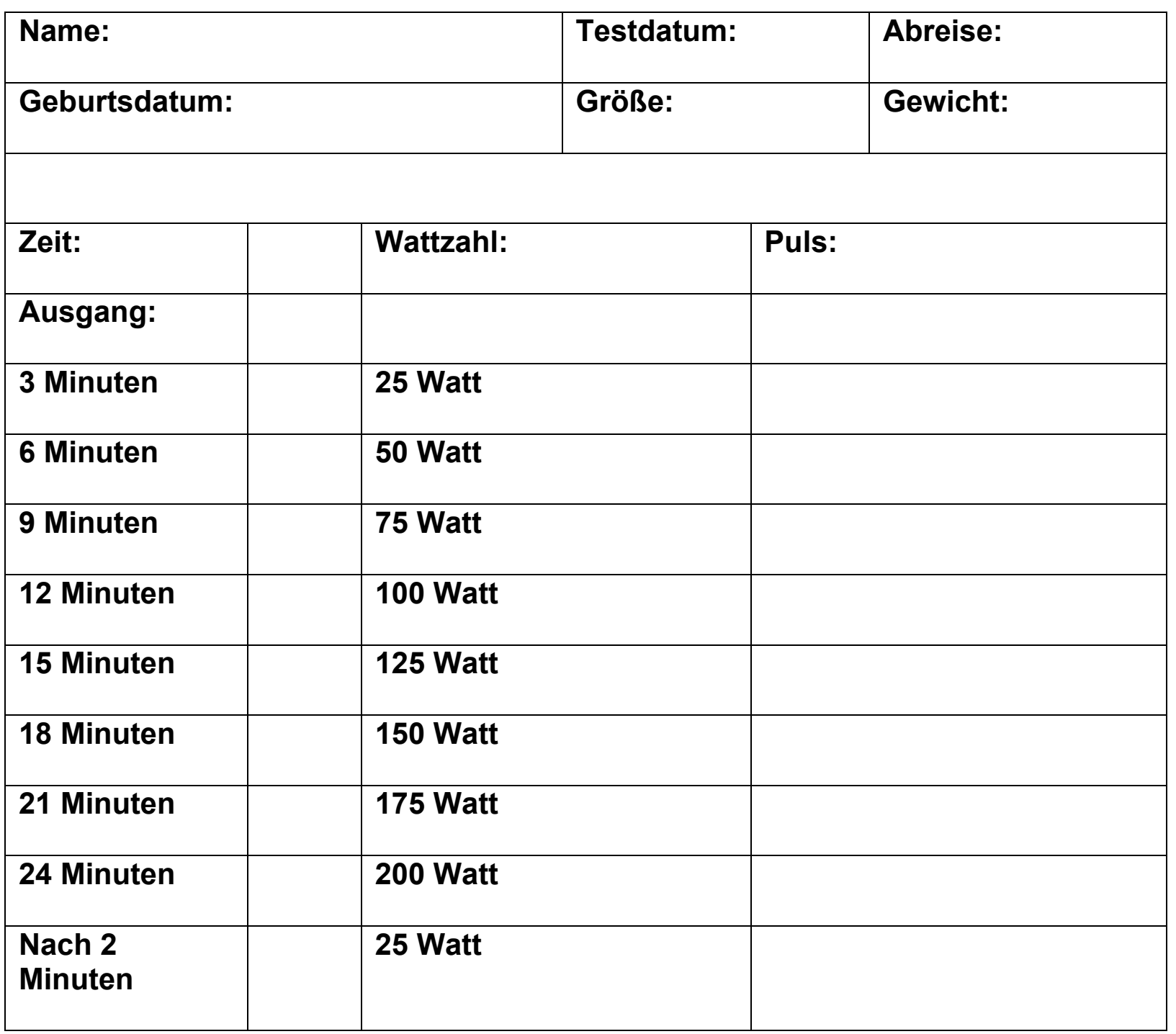




\section{Anlage 10: Dokumentationsbögen zum Übungsmanual}

\section{Dokumentationsbogen zum Übungsmanual}

Liebe Teilnehmer/Teilnehmerinnen,

bitte benutzen Sie die anhängenden Dokumentationsbögen um Ihr Training zu dokumentieren. Tragen Sie hierzu in die Tabelle die Anzahl der Sätze und die jeweiligen Wiederholungszahlen ein (z. B. $3 \times 12$ d. h. 3 Sätze mit je 12 Wiederholungen oder 10, 10, 8 d. h. 1. Satz 10 Wiederholungen, 2. Satz 10 Wiederholungen und 3. Satz 8 Wiederholungen), die Sie am entsprechenden Tag zu jeder Übung gemacht haben. Ergänzen Sie weiterhin auch die Uhrzeit, zu der Sie das Training begonnen haben. Die Vorgaben zum Training können Sie dem Übungsmanual entnehmen. Bitte füllen Sie auch die weiterführenden Angaben über eventuelle sportliche Zusatzaktivitäten aus. Scheuen Sie sich nicht auch längere Spaziergänge oder Wanderungen einzutragen.

Falls Ihnen die vorgegebenen Wiederholungszahlen für einzelne Übungen zu leicht erscheinen, können Sie die Intensität weiter steigern indem Sie die Wiederholungszahl weiter erhöhen oder die Ausführung verlangsamen.

Überschreiten Sie jedoch während des Trainings nicht lhre eigene Belastungsgrenze. Nach dem Training sollte nie ein Gefühl völliger Erschöpfung vorliegen, sondern eher ein angenehmes Gefühl körperlicher Beanspruchung.

\section{Vielen Dank für Ihre Mitarbeit und Viel Spaß beim Trainieren!}


Name:

Monat:

Bitte tragen Sie hier zusätzliche sportliche Aktivitäten ein!

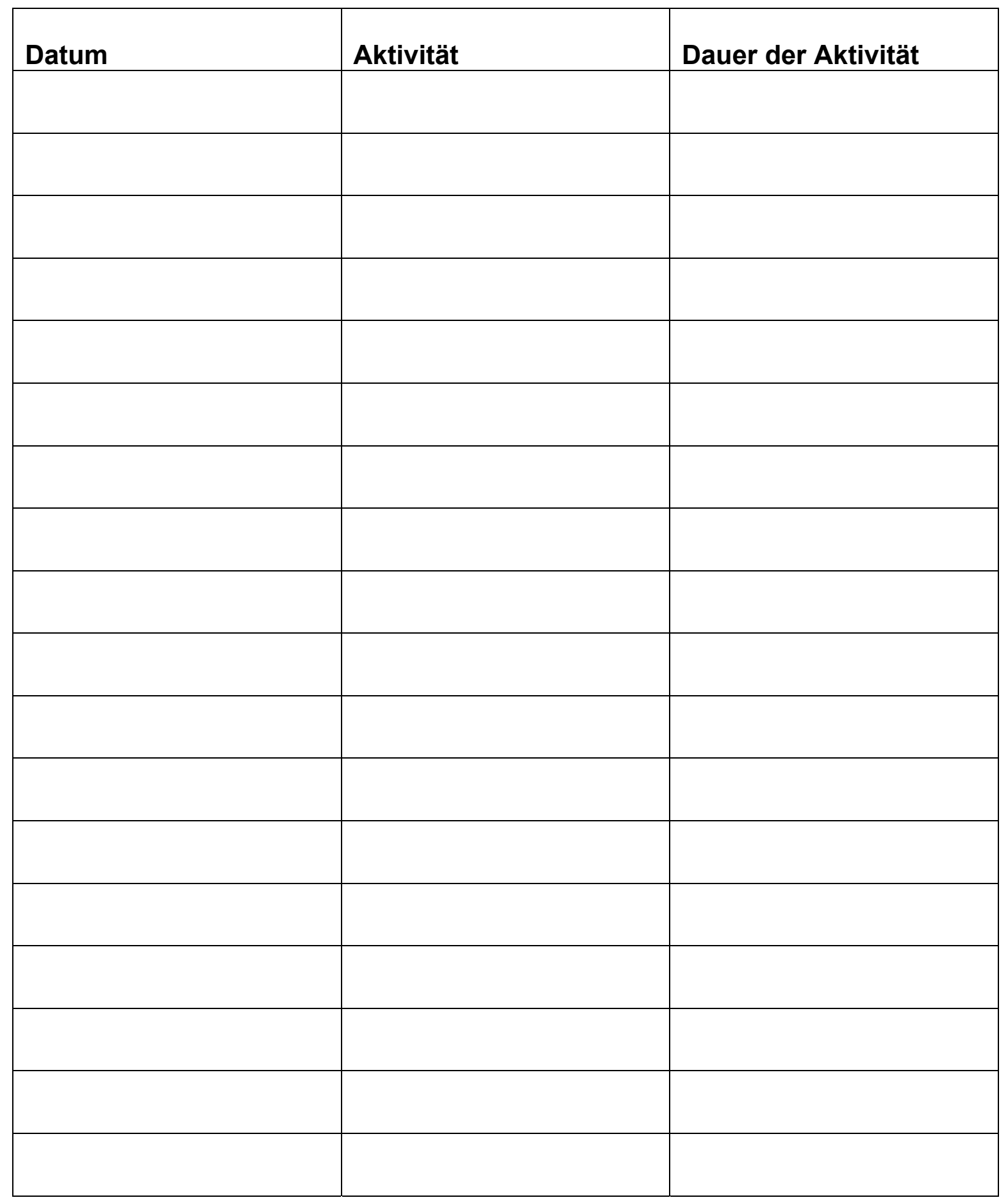


Dokumentationsbogen zum Übungsmanual

Name:

Monat:

\begin{tabular}{|c|c|c|c|c|c|c|c|c|c|}
\hline Datum/Übung & Uhrzeit & Übung 1 & Übung 2 & Übung 3 & Übung 4 & Übung 5 & Übung 6 & Übung 7 & Übung 8 \\
\hline 1 & & & & & & & & & \\
\hline 2 & & & & & & & & & \\
\hline 4 & & & & & & & & & \\
\hline 3 & & & & & & & & & \\
\hline 4 & & & & & & & & & \\
\hline 5 & & & & & & & & & \\
\hline 6 & & & & & & & & & \\
\hline 7 & & & & & & & & & \\
\hline 8 & & & & & & & & & \\
\hline 9 & & & & & & & & & \\
\hline 10 & & & & & & & & & \\
\hline 11 & & & & & & & & & \\
\hline 12 & & & & & & & & & \\
\hline 13 & & & & & & & & & \\
\hline 14 & & & & & & & & & \\
\hline 15 & & & & & & & & & \\
\hline 16 & & & & & & & & & \\
\hline 17 & & & & & & & & & \\
\hline 18 & & & & & & & & & \\
\hline 19 & & & & & & & & & \\
\hline 20 & & & & & & & & & \\
\hline 21 & & & & & & & & & \\
\hline 22 & & & & & & & & & \\
\hline 23 & & & & & & & & & \\
\hline 24 & & & & & & & & & \\
\hline 25 & & & & & & & & & \\
\hline 26 & & & & & & & & & \\
\hline 27 & & & & & & & & & \\
\hline 28 & & & & & & & & & \\
\hline 29 & & & & & & & & & \\
\hline 30 & & & & & & & & & \\
\hline 31 & & & & & & & & & \\
\hline
\end{tabular}


Anlage 11: Übungsmanual für Patienten

Untersuchungsort:

Sonnenberg-Klinik

Bad Sooden-Allendorf

\title{
Übungsmanual zur Doktorarbeit
}

\author{
„Rehabilitatives Krafttraining zur Behandlung \\ tumorbedingter Fatigue unter Einbeziehung des \\ Energieverlaufs der Patienten.“
}

Mattias Heim

Annastraße 16

37075 Göttingen

matze_heim82@web.de

Tel.: 0176/62886040 


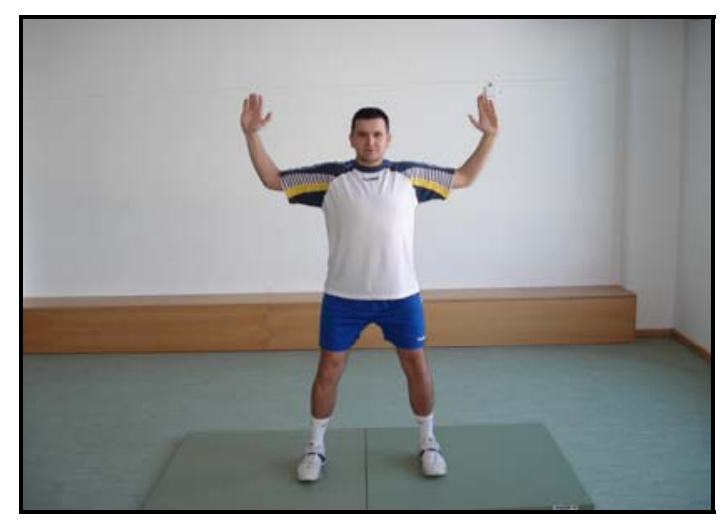

\section{Übung 1: Kniebeugen}

- Füße schulterbreit auseinander

- Arme sind im $90^{\circ}$ Winkel über dem Kopf

- Schulterblätter zusammendrücken

- Hüfte und Po sinken nach hinten unten

- Knie schieben nicht über die Fußspitzen

- Endposition maximal 90 Winkel im Kniegelenk

- Kontrolliert in Ausgangsposition zurückkehren

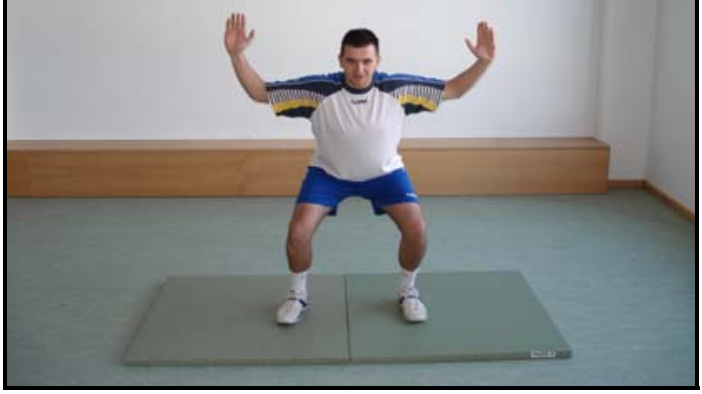

Wiederholungen: $12-15$

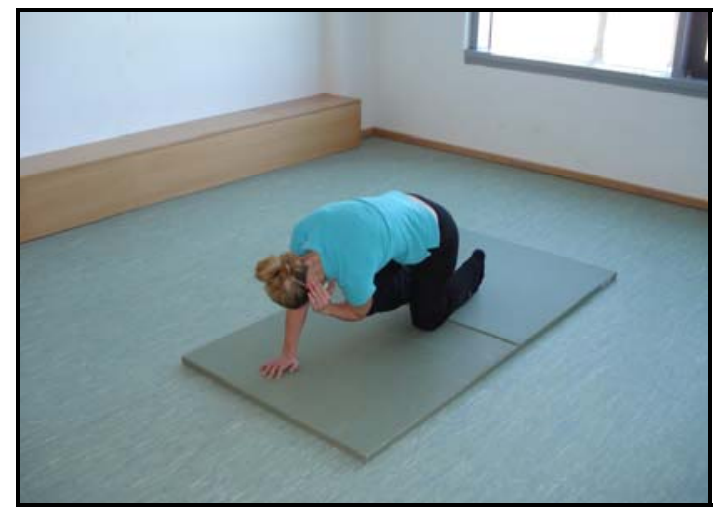

\section{Übung 2: Crunch kniend}

- Knie unterhalb der Hüfte

- Anderes Bein gestreckt

- Fußspitze anziehen

- Ein Arm stabilisiert unterhalb der gegenüberliegenden Schulter

- Die andere Hand befindet sich an der Schläfe

- Aus der eingerollten Position Schulter öffnen und Rumpf zur gegenüberliegenden Seite rotieren

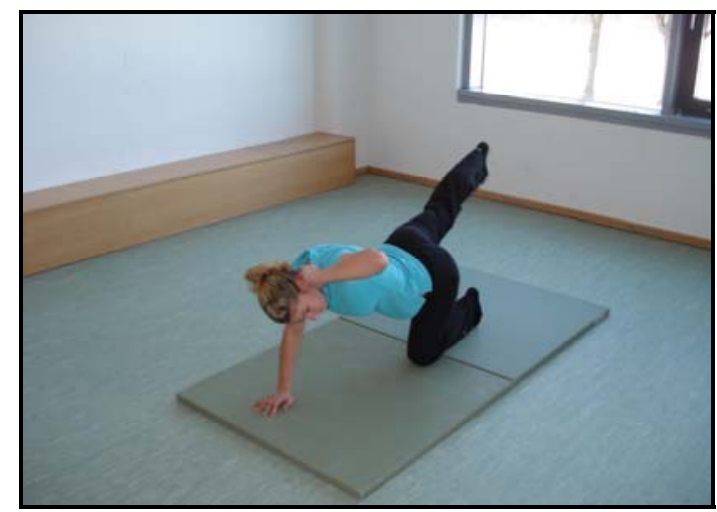

Wiederholungen: $10-12$ pro Seite 


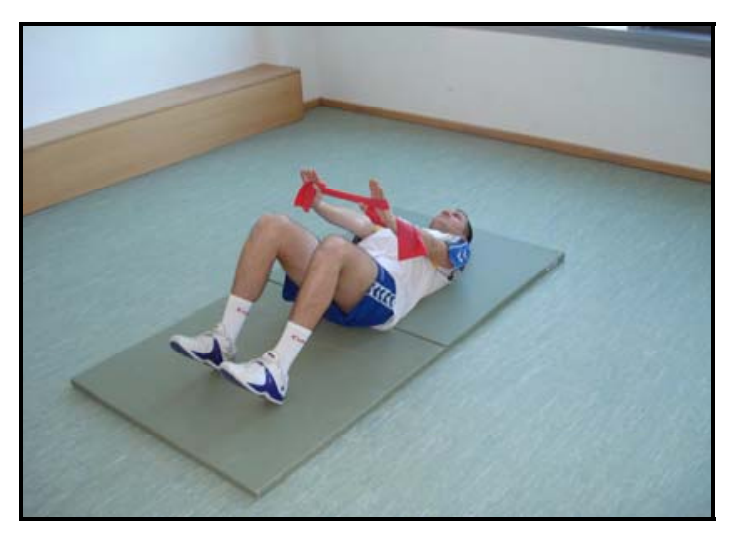

\section{Übung 3: Sit Up's}

- Rückenlage, Beine im 90 Winkel angestellt

- Oberkörper wird langsam mit gestreckten Armen aufgerichtet

- Thera-Band unter Spannung halten

- Unterer Rücken auf dem Boden

- Kopf bildet mit Wirbelsäule eine Linie

- Kontrolliert in Ausgangsposition

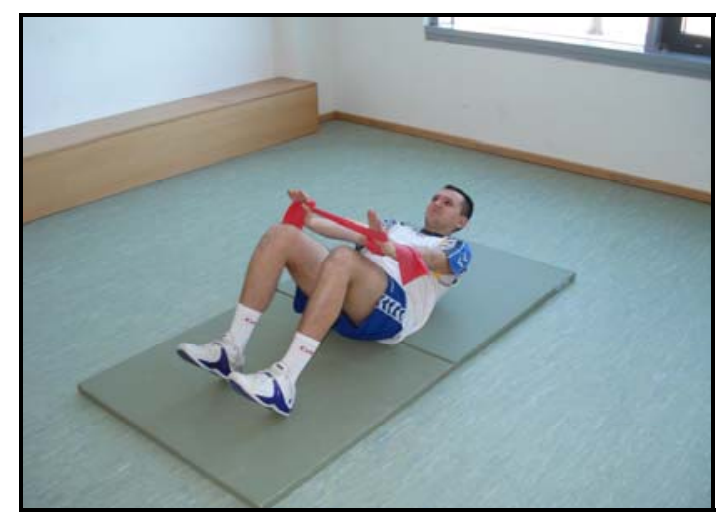
zurückkehren

Wiederholungen: 12 - 15

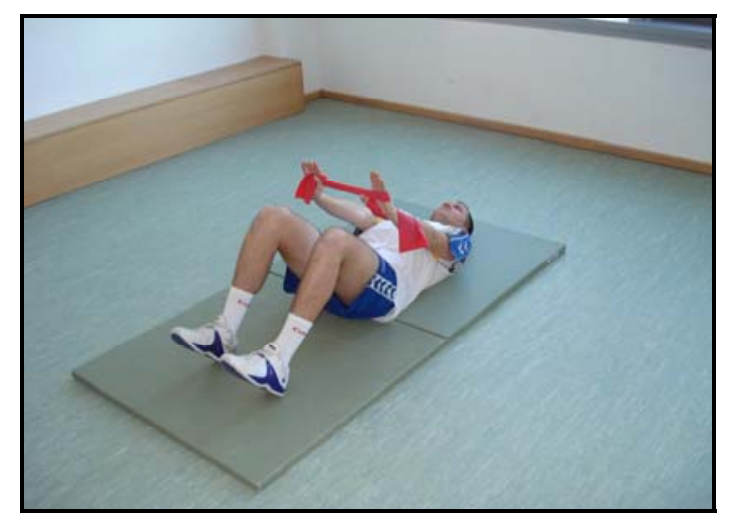

\section{Übung 4: Schräge Sit Up's}

- Rückenlage, Beine im 90 Winkel angestellt

- Oberkörper mit gestreckten Armen abwechselnd nach links und rechts oben einrollen

- Thera-Band unter Spannung halten

- Unterer Rücken auf dem Boden

- Kopf bildet mit Wirbelsäule eine Linie

- Kontrolliert in Ausgangsposition zurückkehren

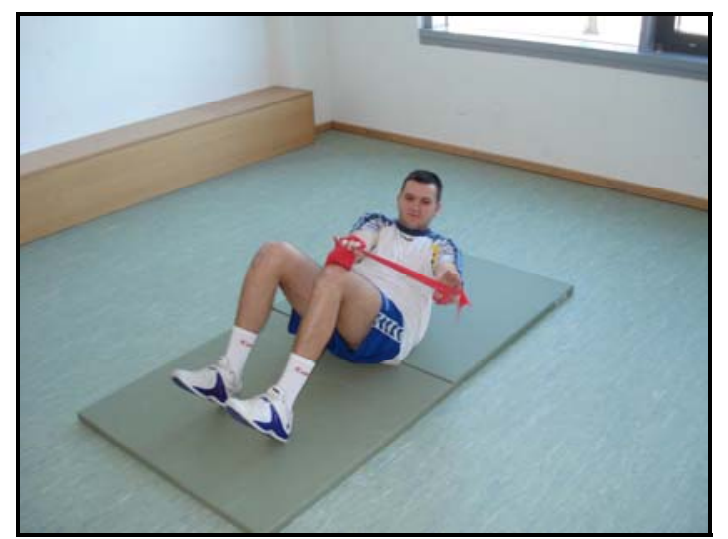

Wiederholungen: 8 - 10 pro Seite 


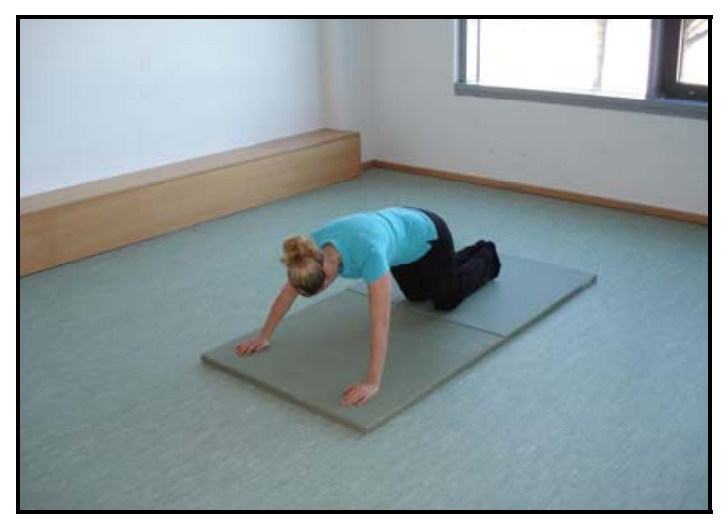

\section{Übung 5: Liegestütz}

- Hände unterhalb der Schultern

- Knie schräg unterhalb der Hüfte

- Beugung der Arme bis Kopf kurz vor dem Boden

- Kopf mit Wirbelsäule in einer Linie

- Kontrolliert in Ausgangsposition zurückkehren

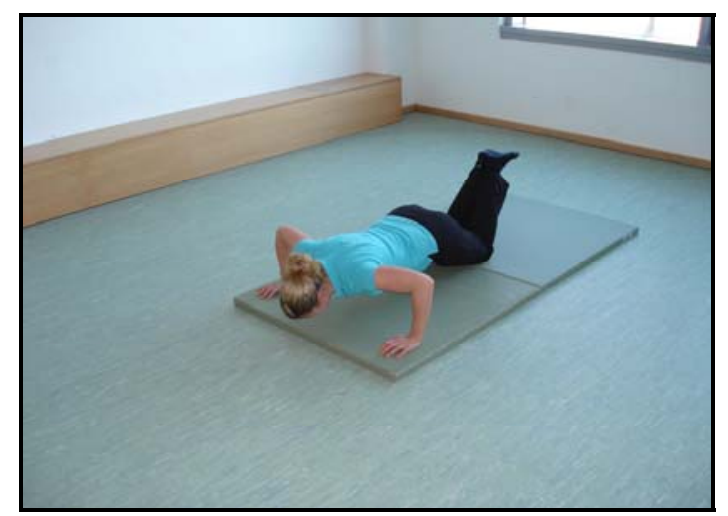

Wiederholungen: $10-12$

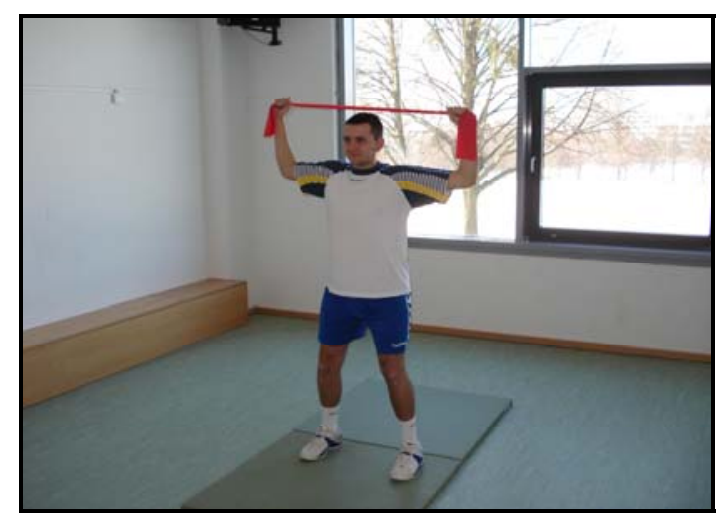

\section{Übung 6: Latziehen}

- Füße stehen etwa Schulterbreit, Knie leicht gebeugt

- $\quad 90^{\circ}$ Winkel im Ellenbogen

- Thera-Band unter Spannung

- Arme hinter dem Kopf nach unten ziehen

- Schulterblätter zusammen drücken

- Kontrolliert in Ausgangsposition zurückkehren

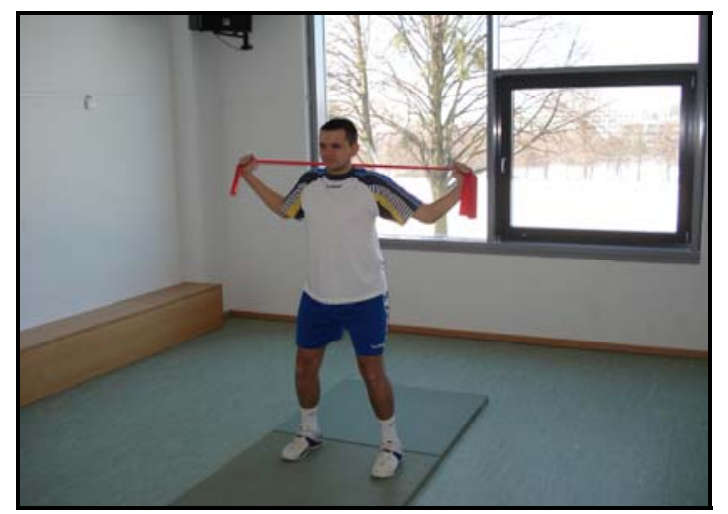

Wiederholungen: $10-12$ 

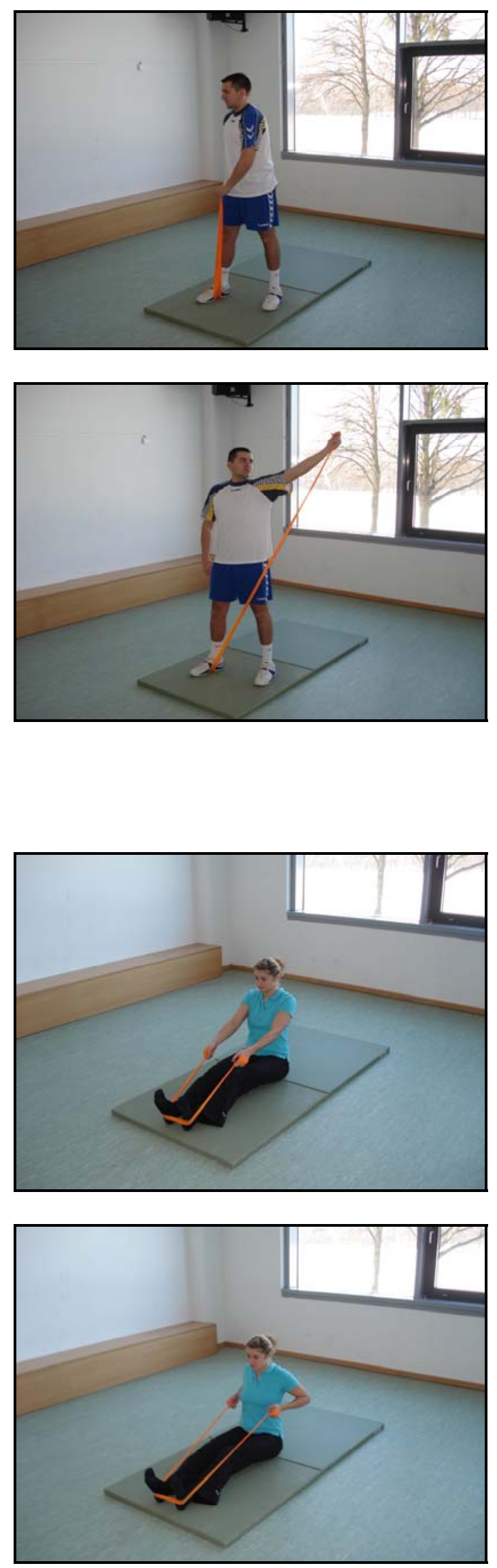

\section{Übung 7: Seitheben}

- Schrittstellung

- Fuß auf Thera-Band

- Oberkörper ist leicht nach innen rotiert

- Arm seitlich am Körper gestreckt anheben

- Blick folgt der Hand

- Kontrolliert in Ausgangsposition zurückkehren

Wiederholungen: $8-10$ pro Seite

\section{Übung 8: Rudern}

- Knie leicht gebeugt

- Hände halten Thera-Band

- Arme werden gebeugt und Thera-Band wird Richtung Bauchnabel gezogen

- Kontrolliert in Ausgangsposition zurückkehren

Wiederholungen: 10 - 12 


\section{Anlage 12: Definition Trainingstermini nach Grosser et al.}

In dieser Anlage erfolgt eine Definition der wichtigsten Trainingstermini und Belastungsnormative.

Belastungsnormative: Sind maßgebliche Größen zur Festlegung der Therapiebelastung. Folgende Normative beinhaltet die Belastung im Einzelnen:

Belastungsintensität, Belastungsdauer, Belastungshäufigkeit, Belastungsumfang, Belastungsdichte, Therapiehäufigkeit

Belastungsintensität: Ist die Ausprägung eines einzelnen Reizes oder einer Reizserie als der Anstrengungs- oder Einsatzgrad einer Übung. Einige Autoren bezeichnen die Belastungsintensität als Krafteinsatz pro Zeiteinheit.

Belastungsdauer: Kennzeichnet die Zeit, in der ein einzelner Therapieinhalt oder eine Serie von Therapieübungen auf den Organismus einwirkt. Die Belastungsdauer lässt sich als Zeitmerkmal genau erfassen. Letztlich wird die Zeitdauer unmittelbar aufeinander folgender Reize als Belastungsdauer bezeichnet.

Belastungsdichte: Ist die Aufeinanderfolge einzelner Übungen oder Serien. Eine hohe Belastungsdichte liegt bei kurzen Pausen vor, eine niedrige bei langen Pausen. Sie bezeichnet also das zeitliche Verhältnis von Belastungs- und Erholungsphasen innerhalb einer Therapieeinheit.

Belastungshäufigkeit: Bezieht sich auf die Anzahl der Einzelreize pro Serie oder pro Therapieeinheit.

Belastungsumfang: Bezeichnet die Summe aller Belastungseinzelreize pro Therapieeinheit.

Therapiehäufigkeit: Gibt die Anzahl der wöchentlichen Therapieeinheiten an. Sie bezeichnet also das zeitliche Verhältnis von Belastungs- und Erholungsphasen zwischen den einzelnen Therapieeinheiten. 
Die hier definierten Belastungsnormative wurden von Radlinger übernommen (RADLINGER 1998).

Trainingsinhalt: Tätigkeit, die im Training ausgeübt wird, um die Trainingsziele zu erreichen. Die sinnvollste Unterteilung ist die nach der Bewegungsstruktur im Vergleich zur Disziplinbewegung. Es wird unterschieden in Allgemein entwickelnde Übungen, Spezialübungen und Wettkampfübungen.

Trainingsmethode: Planmäßiges Verfahren, dass gemäß dem Trainingsziel die Trainingsinhalte, Trainingsmittel und die Belastungsweise festlegt. Eigenständige Grundmethoden haben sich vor allem im Konditionsbereich herauskristallisiert.

Trainingsart: Ausrichtung des Trainings auf die Komponenten der sportlichen Leistung (Kondition, Technik, Taktik) und deren Elemente.

Trainingsform: Verbindung von Trainingsinhalt mit einer bestimmten Belastungsmethode.

Trainingsmittel: Geräte oder Maßnahmen, die den Trainingsablauf unterstützen. Übersichtshalber kann die Vielzahl der zum Einsatz kommenden Mittel noch gegliedert werden in solche organisatorischer Art, informativer Art und gerätemäßiger Art.

Trainingsplanung: Ein „Vorgriff auf die Zukunft“, ein vorausschauendes, systematisches Verfahren, dass charakterisiert ist durch die fortlaufende Anpassung an die Trainings- und Wettkampfwirklichkeit, an den Aufbau in zeitliche Phasen, an die Möglichkeiten der Periodisierung und somit auch durch die Biorhythmik und physiologischen Leistungskurven des Menschen.

Trainingsstufe: Zeitlicher Abschnitt im langfristigen Trainingsaufbau. Nach allgemeiner Zielsetzung wird in Grundlagen-, Aufbau-, Anschluss- und Hochleistungstraining unterschieden. Erfahrungsgemäß erstreckt sich jede Stufe auf 2-4 Jahre, mitunter auch länger. 
Trainingszyklus: Planungsabschnitt vom zeitlich umfangreichsten (Jahre), über mittlere (Makro-, Mikrozyklus) bis zum kleinsten (Tag).

Trainingseinheit: Ein inhaltlich, zeitlich und organisatorisch geschlossenes Ganzes. Ihre Struktur unterliegt meist einer Dreiteilung: Aufwärmen, Hauptteil, Cool Down.

Trainingskontrolle: Erfassen des Trainingszustandes bzw. einzelner Komponenten mittels sportmotorischer, physiologischer, biomechanischer, biochemischer und Beobachtungsverfahren (Test).

Trainingsdokumentation: Registrierung, Protokollierung der Trainings- und Wettkampfleistungen und der situativen Einflüsse.

Trainingsalter: der Zeitraum in Jahren, den man bereits trainiert hat.

Trainingsmaßnahmen: Trainingsbegleitende Aspekte wie Wiederherstellungsmaßnahmen, Aufwärmen, Abwärmen, Bekleidung usw.

Trainingsanwendungsbereich/Trainingsklasse: Alters- bzw. entwicklungsstufenangepasster Trainingsabschnitt, der den biologisch-motorischen Entwicklungsstand und das geistig-seelische Niveau bei der Auswahl von Trainingszielen, Trainingsinhalten und Trainingsmethoden berücksichtigt. Demnach wird Kinder-, Jugend- und Erwachsenentraining unterschieden.

Trainingsziel: Zielsetzung des Trainings. Je nach Grad der Verallgemeinerung kann ein Trainingsziel unterschiedlich formuliert sein. Es wird unterschieden in Übergeordnete Ziele (Grobziele), Teilziele (Feinziele) und Zielfaktoren (Feinstziele).

Die hier definierten Trainingstermi wurden von Grosser et al. übernommen (GROSSER et al. 2008). 


\section{Anlage 13: Trainingsprinzipien nach Grosser et al.}

\section{Prinzip des Wirksamen Belastungsreizes:}

Dieses Prinzip sagt aus, dass der Trainingsreiz eine bestimmte Intensitätsschwelle überschreiten muss, um überhaupt eine Anpassungsreaktion auszulösen, d. h. um trainingswirksam zu sein. Biologischer Hintergrund ist die Reizstufenregel, nach der im Hinblick auf funktionelle und morphologische Anpassungsänderungen unterschwellige, überschwellig schwache, überschwellig starke und zu starke Reize unterschieden wird. Unterschwellige Reize bleiben wirkungslos, unterschwellig starke Reize erhalten das Funktionsniveau, überschwellig starke Reize lösen physiologische und anatomische Änderungen aus und zu starke Reize schädigen die Funktion. Der Schwellenwert hängt vom Leistungszustand des Trainierenden ab.

\section{Prinzip der progressiven Belastungssteigerung:}

Wenn Trainingsbelastungen über eine längere Zeitdauer gleich bleiben, hat sich der Organismus so angepasst, dass dieselben Belastungsreize nicht mehr überschwellig stark oder sogar unterschwellig werden. Sie rufen keine weitere Leistungssteigerung hervor. Die Konsequenz daraus ist die fortschreitende Steigerung der Trainingsbelastung in gewissen Zeitabständen. Je nach biologischem Alter, Trainingsalter und sportmotorischem Entwicklungsniveau erfolgt die Steigerung individuell allmählich. Eine allmähliche Steigerung ist solange sinnvoll, wie noch eine Leistungssteigerung festzustellen ist. Stagniert diese oder kommt es sogar zu Rückschritten ist Vorsicht geboten, da biologische Anpassungsvorgänge mitunter längere Zeiträume in Anspruch nehmen können.

\section{Prinzip der Variation der Trainingsbelastung:}

Im Rahmen wirksamer Trainingsbelastungen darf die Rolle des sympathischen vegetativen Nervensystems (Sympathikus) nicht übersehen werden. Der Sympathikus versetzt den Körper in einen Zustand hoher Leistungsbereitschaft, was notwendig für wirksame Trainingsbelastung ist. Bei ständig gleichartiger Stimulierung unterliegt er gewissermaßen auch der Reizstufenregelung und es zeigt sich eine Abnahme seines leistungssteigernden Effekts. Gleichbleibende Reize über einen gewissen Zeitraum führen daher zu einer Stagnation des Trainingsgewinns. Durch Änderung des Belastungsreizes kann die vorherige Stimulationslage wieder erreicht 
werden. Diese Variation der Belastungsreize hat sich im praktischen Trainingsgeschehen nicht nur auf Intensitätsänderungen, sondern vor allem auf den Wechsel von Trainingsinhalten, der Bewegungsdynamik, der Pausengestaltung, also auch der Trainingsmethoden zu beziehen. Sie stellen für den angesprochenen Bereich eine Unterbrechung der Belastungsmonotonie dar und verursachen als ungewohnte Belastungsreize weitere Homöostasestörungen mit nachfolgender Anpassung.

\section{Prinzip der optimalen Gestaltung von Belastung und Erholung:}

Dieses Grundprinzip berücksichtigt, dass nach einer wirkungsvollen Trainingsbelastung eine gewisse Zeit der Wiederherstellung notwendig ist, um eine erneute gleichartige Belastung bei günstigen Voraussetzungen durchführen zu können. Belastung und Erholung sind gewissermaßen als Einheit zu betrachten. Biologische Grundlage ist das Prinzip der Superkompensation, demzufolge es nach einem entsprechend starken Belastungsreiz nicht nur zur Wiederherstellung (Kompensation), sondern zu einer erhöhten Wiederherstellung (Überkompensation) der beanspruchten Energiespeicher (Kreatinphosphat, Glykogen) kommen kann. Das erhöhte Niveau bleibt allerdings nicht dauerhaft erhalten, sondern bildet sich nach 13 Tagen bei Anfängern wieder zurück. Es muss jedoch angemerkt werden, dass sich diese Modellvorstellung lediglich auf die Entleerung der genannten muskulären Energiespeicher bezieht. Adaptionsvorgänge auf zellulärer Ebene lassen sich mit der Modellvorstellung der Superkompensation nicht erklären.

\section{Prinzip der Wiederholung und Dauerhaftigkeit (Kontinuität):}

Zum Erreichen einer optimalen Anpassung ist es notwendig, mehrfach die Belastung zu wiederholen, da für eine stabile Adaption der Organismus zunächst eine Reihe von akuten Umstellungen einzelner Funktionssysteme durchlaufen muss. Die endgültige Adaption ist erst erreicht, wenn über die Anreicherung von Substraten hinaus auch in anderen Funktionssystemen Umstellungen erfolgten und vor allem auch das Zentralnervensystem als Führungsinstanz von Bewegungsleistungen sich angepasst hat. 


\section{Prinzip der Individualität und Altersgemäßheit:}

Das Prinzip der Individualität und Altersgemäßheit bezieht sich auf die Heterogenität des einzelnen Individuums. In jeder Trainingsplanung muss der individuelle Ausgangszustand des Sportlers beachtet werden. Dabei spielen Faktoren wie Alter, Trainingsalter oder individuelle genetische maximale Funktionskapazität eine zentrale Rolle. Zudem hat jeder Mensch eine individuelle Adaptionsfähigkeit. Diese zu kennen, ist essenziell für eine effektive Trainingsgestaltung ist.

Die beschriebenen Trainingsprinzipien wurden von Grosser et al. 2008 veröffentlicht (GROSSER et al. 2008). 


\section{Anlage 14: Adaptionsstufen nach Grosser et al.}

Stufe 1: Veränderung des motorischen Steuerprogramms 7.-10. Tage.

Beobachtet werden kann ein verbesserter Bewegungsablauf, die muskuläre Ermüdung setzt später ein, die Energieversorgung belasteter Muskelgruppen verbessert sich und die Rekrutierung von schnell und langsam kontrahierenden Muskelfasern passt sich den Erfordernissen der Belastung an.

Stufe 2: Vergrößerung der Energiespeicher 10.-20. Tage.

Die Engpasssituation „anhaltender Glykogenmangel“ ist auch als auslösender Reiz für die Zunahme und Umverteilung dieses Energiespeichers anzusehen; die Zunahme der Energiespeicher benötigt nun mehr Raum in der Muskelfaser. Wenn dann im Ausdauertraining eine Widerstandskomponente (Kraftausdauertraining) hinzukommt, ist eine wesentliche physiologische Voraussetzung für die Muskelhypertrophie gegeben. Für die Erhöhung der muskulären Leistungsfähigkeit sind der Strukturverschleiß durch das wiederholte Training (belastungsbedingter Proteinverlust) und der damit verbundene Energiemangel die entscheidenden Reize. Zur Vergrößerung der Energiespeicher und zur Einleitung der Muskelhypertrophie wird ein Zeitraum von mindestens 20 Tagen als notwendig erachtet.

Stufe 3: Optimierung geregelter Systeme und Strukturen 20.-30. Tage.

Die Veränderung der Muskulatur in ihrem Aufbau macht die Erarbeitung eines neuen Gleichgewichts mit den ansteuernden motorischen Einheiten erforderlich, die Muskelfasern müssen adäquat zur sportartspezifischen Belastung aktiviert werden. Dieser störanfällige Prozess findet in der Regel zwischen der 3. und 4. Trainingswoche statt und erfordert eine deutliche Senkung der Belastung. Die Änderung der Trainingsbelastung nach etwa drei Wochen - empfohlen wird, die Gesamtbelastung um mindestens $30 \%$ zu vermindern - führt zu einem geringeren Gesamtenergieverbrauch und erleichtert die Reizverarbeitung in der 4. Woche.

Stufe 4: Koordinierung der Hierarchie der Systeme 30.-40. Tag

Wenn sich die zentralen Steuerhierarchien (Neuroendokrinum, Vegetativum, und Immunsystem) mit dem neuen Zustand in der Muskulatur abgestimmt haben, ist für den Athleten ein vorübergehend stabiler Zustand erhöhter, effektiverer 
Leistungsfähigkeit erreicht. Dieser zentrale und periphere Funktionsabgleich ist ein zeitgebundener Prozess und benötigt etwa zwei Wochen bis zu seiner Vervollkommnung; die Koordinierung der Systeme ist somit nach etwa 40 Tagen abgeschlossen. Ist diese Anpassungsstufe individuell verfügbar, vermindert sich der biologische Aufwand zur Belastungsbewältigung, wie die Messungen von Herzfrequenz, maximaler Sauerstoffaufnahme und Laktat belegen. 
Eidesstaatliche Erklärung:

„Ich versichere, dass ich die eingereichte Dissertation „Rehabilitatives Krafttraining zur Behandlung tumorassoziierter Fatigue unter Einbeziehung zirkadianrhythmischer Aspekte während und nach stationärer onkologischer Rehabilitation" selbstständig und ohne unerlaubte Hilfsmittel verfasst habe. Anderer als der von mir angegebenen Hilfsmittel und Schriften habe ich mich nicht bedient. Alle wörtlich oder sinngemäß den Schriften anderer Autoren entnommenen Stellen habe ich kenntlich gemacht."

Lebenslauf:

Name: $\quad$ Matthias Heim

18.07.1982 in Eisenach

Familienstand: ledig

\author{
Ausbildung: \\ 08/1993 - 07/2001 Elisabeth Gymnasium Eisenach \\ Erwerb der allgemeinen Hochschulreife \\ 10/2002 Georg-August-Universität Göttingen \\ Beginn des Studiums in Sportwissenschaften und Mathematik \\ 08/2008 Georg-August-Universität Göttingen \\ 1. Staatsexamen für das Lehramt an Gymnasien in \\ Sportwissenschaften und Mathematik \\ 10/2008 - 02/2011 Georg-August-Universität Göttingen \\ Promotion \\ ab 02/2011 Studienseminar Göttingen/ \\ Eichsfeld-Gymnasium Duderstadt \\ Vorbereitungsdienst für das Lehramt an Gymnasien
}

Göttingen, den 


\section{Danksagung:}

Mein Dank für die hilfreiche Unterstützung und Betreuung bei der Erstellung dieser Arbeit gilt Prof. Dr. Dr. A. Niklas, Prof. Dr. M. Heim und Prof. Dr. A. Krüger. Im speziellen möchte ich mich bei Prof. Dr. Heim für die Möglichkeit der Durchführung einer Studie an der Sonnenberg-Klinik Bad Sooden Allendorf bedanken. In diesem Zusammenhang gilt mein Dank auch allen Mitarbeitern der Sonnenberg-Klinik, die mir während der Datenerhebung immer mit Rat und Tat zur Seite gestanden haben. Weiterhin möchte ich mich bei meinen Kollegen des Instituts für Sportwissenschaften der Universität Göttingen bedanken. Hier gilt mein ausdrücklicher Dank Dr. Markus Wuttke für zahlreiche fachspezifische und private Gespräche und einer Zusammenarbeit die zu einer guten Freundschaft geworden ist. Meinem Schwager Thorsten danke ich für die Unterstützung beim Druck dieser Arbeit.

In besonderem Maße möchte ich mich bei meinen Eltern, meiner Familie und meiner Partnerin bedanken, ohne deren Unterstützung mir eine solche Arbeit niemals möglich gewesen wäre. Vielen Dank für eure Unterstützung in allen Bereichen meines Lebens. 